\title{
WestVirginiaUniversity
}

THE RESEARCH REPOSITORY @ WVU

Graduate Theses, Dissertations, and Problem Reports

2009

\section{Investigation into pedestrian exposure to near-tailpipe exhaust emissions}

Neil A. Buzzard

West Virginia University

Follow this and additional works at: https://researchrepository.wvu.edu/etd

\section{Recommended Citation}

Buzzard, Neil A., "Investigation into pedestrian exposure to near-tailpipe exhaust emissions" (2009). Graduate Theses, Dissertations, and Problem Reports. 2037.

https://researchrepository.wvu.edu/etd/2037

This Thesis is protected by copyright and/or related rights. It has been brought to you by the The Research Repository @ WVU with permission from the rights-holder(s). You are free to use this Thesis in any way that is permitted by the copyright and related rights legislation that applies to your use. For other uses you must obtain permission from the rights-holder(s) directly, unless additional rights are indicated by a Creative Commons license in the record and/ or on the work itself. This Thesis has been accepted for inclusion in WVU Graduate Theses, Dissertations, and Problem Reports collection by an authorized administrator of The Research Repository @ WVU. For more information, please contact researchrepository@mail.wvu.edu. 


\title{
Investigation into Pedestrian Exposure to Near-Tailpipe Exhaust Emissions
}

\author{
Neil A. Buzzard \\ Thesis submitted to the \\ College of Engineering and Mineral Resources \\ at West Virginia University \\ in partial fulfillment of the requirements \\ for the degree of
}

Master of Science

in

Mechanical Engineering

Department of Mechanical and Aerospace Engineering

\author{
Nigel N. Clark, Ph.D., chair \\ Steven E. Guffey, Ph.D. \\ Benjamin C. Shade, Ph.D.
}

Morgantown, West Virginia

2009

Keywords: Diesel Exhaust, Particulate Matter, Near-Roadway, Exposure

Copyright 2009 Neil A. Buzzard 


\section{Abstract \\ Investigation into Pedestrian Exposure to Near-Tailpipe Exhaust Emissions}

Neil A. Buzzard

Inhalation of particulate matter is known to cause negative human health effects. Consequently, regulatory agencies have set regulations and standards that limit the maximum concentrations to which persons may be exposed and the maximum concentrations allowed in the ambient air. However, these standards consider steady exposure over large spatial and time scales. Because many vehicles' exhaust systems direct exhaust towards sidewalks, pedestrians in close proximity to a roadway may experience events where for brief periods of time particulate matter concentrations are high enough to cause acute health effects. In order to quantify these exposure events, instruments which measure specific exhaust pollutant concentrations were placed near a roadway and connected to the mouth of a mannequin used as a pedestrian surrogate. A representative estimate of the exposure potentially experienced by pedestrians was obtained by measuring concentrations at the mannequin's mouth during drive-by events with a diesel truck and a gasoline truck. Breathing rates were then multiplied by the measured concentrations to determine the mass of pollutant inhaled daily and per breath. The highest concentrations observed with the diesel test vehicle were 2.2 million particles/cc and $1400 \mu \mathrm{g} / \mathrm{m}^{3}$. The average concentration of particulate matter measured over the duration of a single drive-by test was observed to reach the same order of magnitude as the low concentrations used in human clinical studies which are known to cause acute health effects. It was also observed that concentrations of particulate matter were 2 to 3 times higher at the height of a stroller than at the mouth of a standing mannequin during heavy acceleration tests. However, for other operating conditions, the opposite of this result was observed. Additionally, particulate concentrations obtained with the diesel vehicle were typically an order of magnitude or more greater than those obtained with the gasoline vehicle. Particulate matter concentrations during drive-by incidents can easily reach or exceed the low concentrations that can cause acute health effects for brief periods of time. For the case of a 2006 diesel fueled Dodge Ram 2500 and a 2001 gasoline fueled Dodge Ram 1500, the mass of particulate matter inhaled during drive-by incidents was small compared to the mass inhaled daily at ambient conditions. On a per breath basis, however, the mass of particulate matter inhaled was large compared to the mass inhaled at ambient conditions. Finally, it was determined that exposure is directly dependent on the location of a pedestrian with respect to the tailpipe of a passing vehicle. 


\section{Acknowledgements}

Dr. Nigel Clark, many thanks for giving me the opportunity to work with you. None of this would have possible without you. I am grateful for all of the wisdom and advice you have shared with me throughout my graduate career. You have motivated and inspired me to continue striving for success. Dr. Steve Guffey, thank you for your part in this research. Your expertise helped this research come to a more rapid and productive conclusion. I am thankful for all of your advice throughout the creation of this thesis and my journal article.

I thank West Virginia University Research Corporation for program support. All of the staff at the Center for Alternative Fuels, Engines and Emissions deserves many thanks for their valuable support. David McKain and James Perry, thank you for piloting the vehicles throughout testing and your continued support in helping wherever it was needed. Yuebin $\mathrm{Wu}$, thank you for your assistance with the emissions measurement. David Estep and Josh Israel, thank you for having all the analyzers in good working condition. Wayne Hildebrand, thank you for providing a power supply at the sampling location.

Amy, my fiancé, thank you for being patient on all of those late nights and long days and thank you supporting me 100 percent of way. Without your encouragement, I do not know if I ever would have made it to this point. Lastly, I thank my parents for always being there when I needed them and always believing in me. 


\section{Table of Contents}

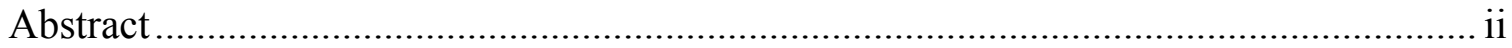

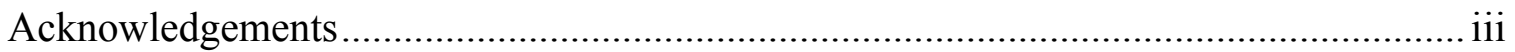

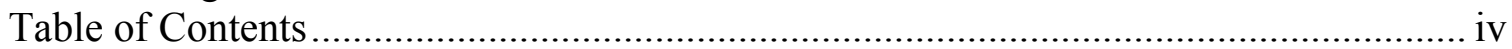

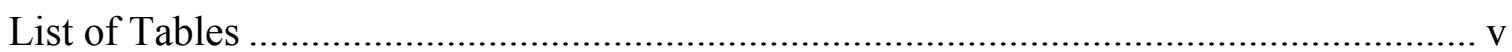

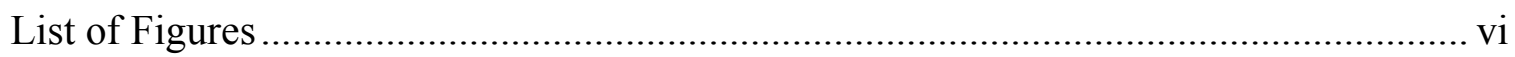

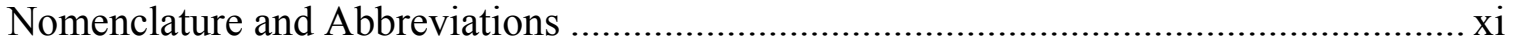

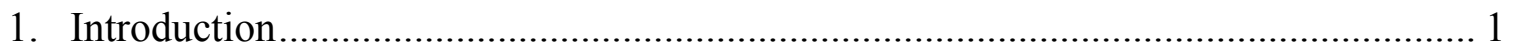

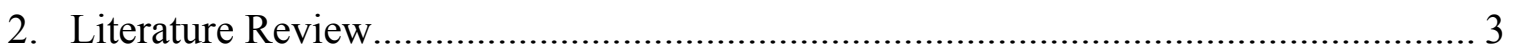

2.1. Particulate Matter......................................................................................... 3

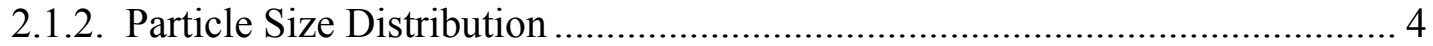

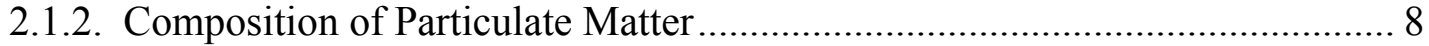

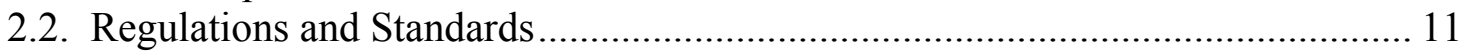

2.3. Health Effects of Particulate Matter .................................................................... 13

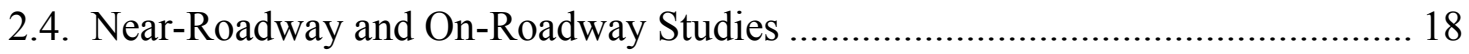

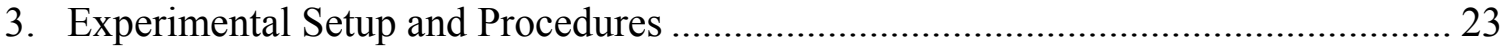

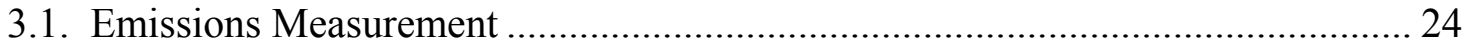

3.1.1. Cambustion DMS500 Fast Particle Spectrometer ...................................... 24

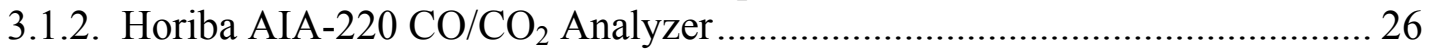

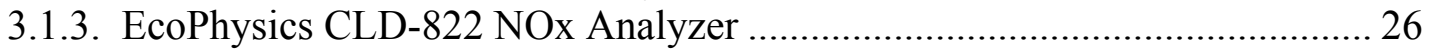

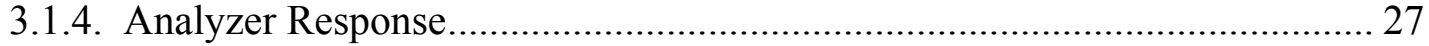

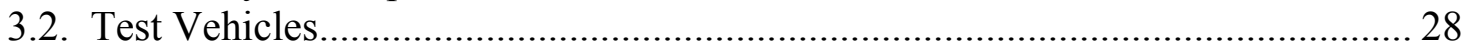

3.3. Engine Control Unit Data Logger....................................................................... 28

3.4. Pedestrian Surrogate and Sample Probe Support .............................................. 29

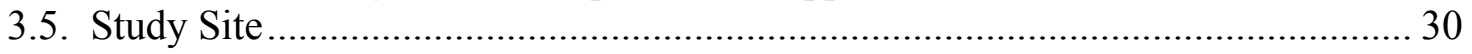

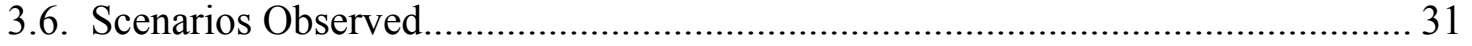

3.7. Pollution Monitoring.......................................................................................... 33

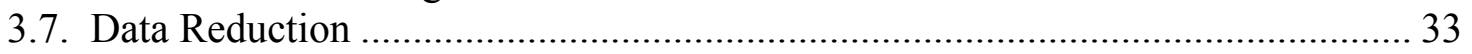

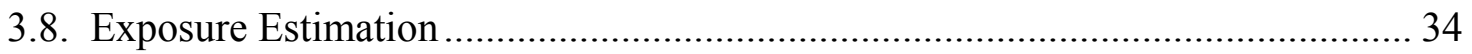

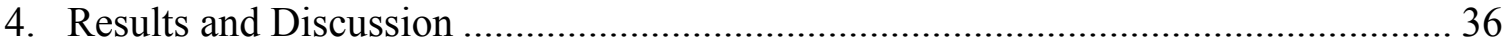

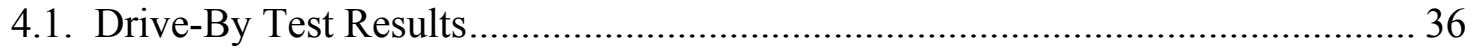

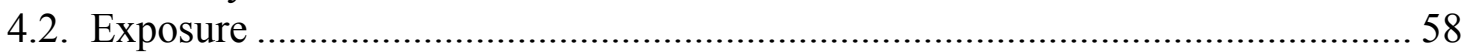

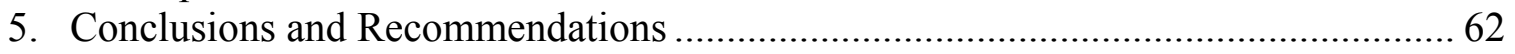

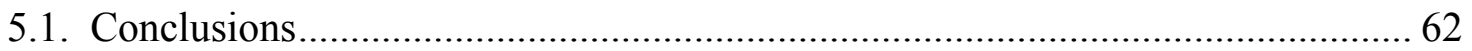

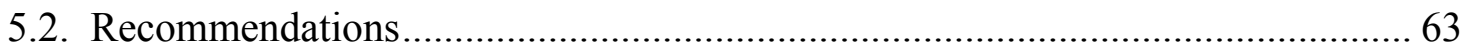

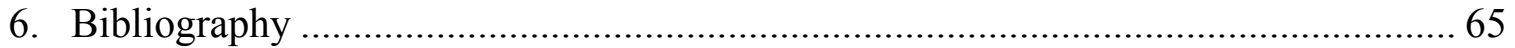

7. Appendix A: Gaseous analyzer regression equations ...................................... A1

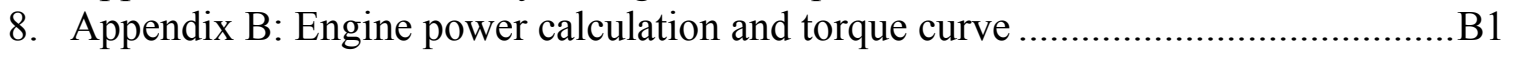

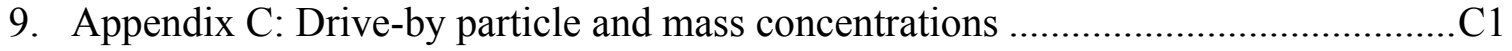

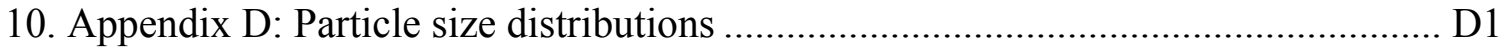




\section{List of Tables}

Table 2-1: National ambient air quality standards [31].

13

Table 4-1: Average incident PM concentrations, durations, and peak concentrations for hard acceleration diesel drive-bys

Table 4-2: Average incident PM concentrations, durations, and peak concentrations for medium acceleration diesel drive-bys.

Table 4-3: Average incident PM concentrations, durations, and peak concentrations for cruising diesel drive-bys

Table 4-4: Average incident PM concentrations, durations, and peak concentrations for hard acceleration gasoline drive-bys. 54

Table 4-5: Average incident PM concentration, durations, and peak concentrations for medium acceleration gasoline drive-bys. 55

Table 4-6: Average incident PM concentrations, durations, and peak concentrations for cruising gasoline drive-bys. 56

Table 4-7: Breathing rates for men, women, and children walking and standing [136]... 59 Table 4-8: Mass inhaled due to 264 average drive-by incidents and daily at different ambient conditions. 59

Table 4-9: Mass inhaled per breath during worst drive-by incident and at different ambient conditions. 60

Table 8-1: Torque vs. engine speed for Dodge Ram 2500. B1 


\section{List of Figures}

Figure 2-1: "Typical engine exhaust mass and number weighted size distribution shown

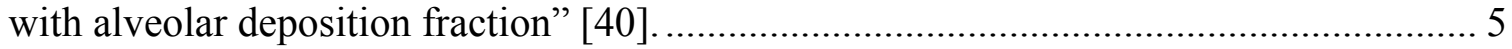

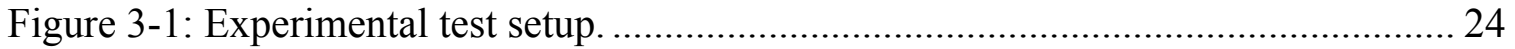

Figure 3-2: Cambustion DMS500 Fast Particle Spectrometer (cambustion.co.uk)......... 25

Figure 3-3: Test vehicles: Dodge Ram 2500 (left) and Dodge Ram 1500 (right)........... 28

Figure 3-4: AutoTap OBDII Diagnostic Scanner. ..................................................... 29

Figure 3-5: Pedestrian surrogate mannequin (left) mannequin's head simulation in stroller

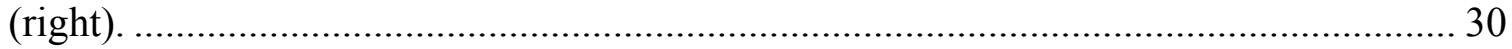

Figure 3-6: Mannequin's head with sample probes in mouth and attachment to sample

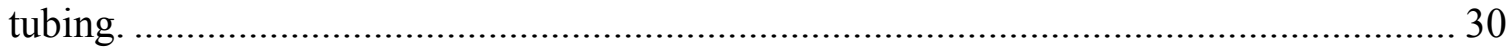

Figure 3-7: Diagram of drive-by experiment layout................................................ 32

Figure 4-1: Raw data from hard acceleration drive-by with mannequin. ....................... 37

Figure 4-2: Raw data from medium acceleration drive-by with mannequin................... 38

Figure 4-3: Raw data from cruising drive-by with mannequin..................................... 38

Figure 4-4: Comparison of mass concentration determination methods. ........................ 39

Figure 4-5: Particle number and mass concentrations for diesel and gasoline vehicle. ... 40

Figure 4-6: Normalized number weighted particle size distribution obtained with the

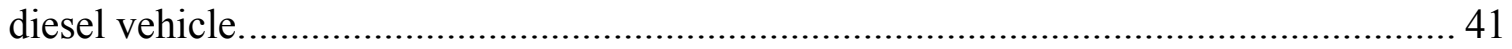

Figure 4-7: Normalized mass weighted particle size distribution obtained with the diesel

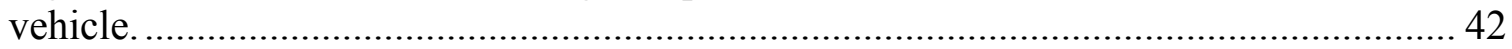

Figure 4-8: Normalized number weighted particle size distribution obtained with the

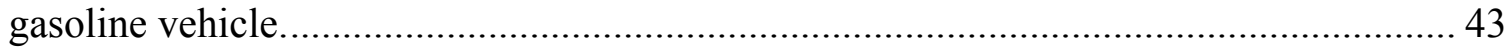

Figure 4-9: Normalized mass weighted particle size distribution obtained with the gasoline vehicle............................................................................................... 44

Figure 4-10: Number weighted particle size distribution obtained with the diesel vehicle.

Figure 4-11: Mass weighted particle size distribution obtained with the diesel vehicle. . 46 Figure 4-12: Number weighted particle size distribution obtained with the gasoline vehicle.

Figure 4-13: Mass weighted particle size distribution obtained with the gasoline vehicle.

Figure 4-14: Average incident PM concentrations for all full load acceleration tests..... 49

Figure 4-15: Average incident durations for all full load acceleration tests.................... 50

Figure 4-16: Comparison of mass inhaled due to 264 drive-bys and mass inhaled daily. 59

Figure 4-17: Comparison of mass inhaled per breath during drive-by and at ambient. ... 61

Figure 7-1: Calibration polynomial regression equation for $\mathrm{CO}$ analyzer. .................... A1

Figure 7-2: Calibration polynomial regression equation for $\mathrm{CO}_{2}$ analyzer.................. A2

Figure 7-3: Calibration polynomial regression equation for NOx analyzer. .................. A2

Figure 8-1: Torque curve for the Dodge Ram 2500. ..................................................

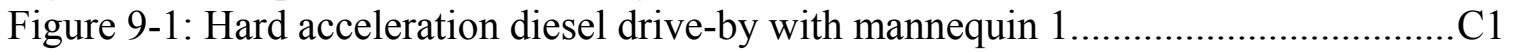

Figure 9-2: Hard acceleration diesel drive-by with mannequin 2 ...................................

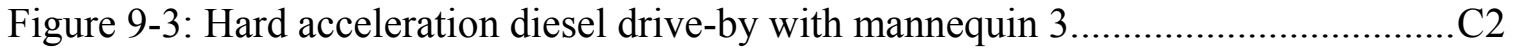

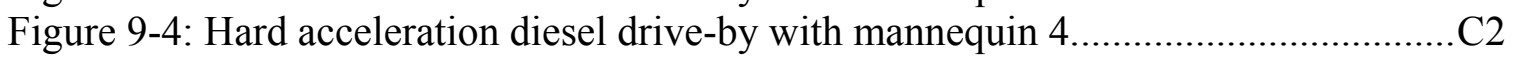

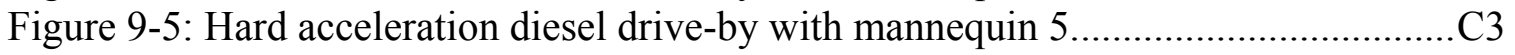


Figure 9-6: Hard acceleration diesel drive-by with mannequin 6....................................... 3

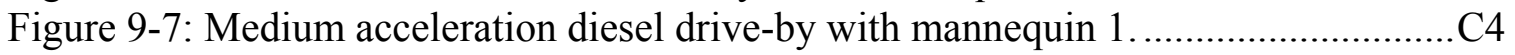

Figure 9-8: Medium acceleration diesel drive-by with mannequin 2...............................

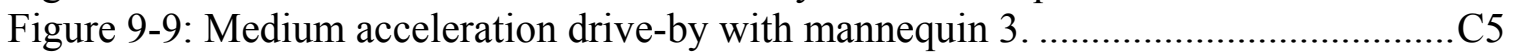

Figure 9-10: Medium acceleration diesel drive-by with mannequin 4.............................. 5

Figure 9-11: Medium acceleration diesel drive-by with mannequin 5............................... 6

Figure 9-12: Medium acceleration diesel drive-by with mannequin 6.............................. 6

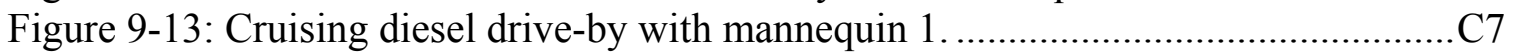

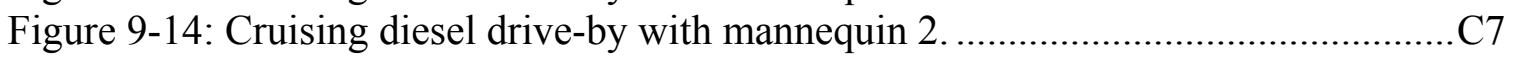

Figure 9-15: Cruising diesel drive-by with mannequin 3 ................................................ 8

Figure 9-16: Cruising diesel drive-by with mannequin 4 ............................................... 8

Figure 9-17: Cruising diesel drive-by with mannequin 5.............................................

Figure 9-18: Cruising diesel drive-by with mannequin 6.............................................. 9

Figure 9-19: Hard acceleration diesel drive-by without mannequin 1............................. 10

Figure 9-20: Hard acceleration diesel drive-by without mannequin 2............................ 10

Figure 9-21: Hard acceleration diesel drive-by without mannequin 3............................ 11

Figure 9-22: Hard acceleration diesel drive-by without mannequin 4. ............................ 11

Figure 9-23: Hard acceleration diesel drive-by without mannequin 5............................ 12

Figure 9-24: Hard acceleration diesel drive-by without mannequin 6............................. 12

Figure 9-25: Medium acceleration diesel drive-by without mannequin 1........................ 13

Figure 9-26: Medium acceleration diesel drive-by without mannequin 2....................... 13

Figure 9-27: Medium acceleration diesel drive-by without mannequin 3.....................C14

Figure 9-28: Medium acceleration diesel drive-by without mannequin 4........................ 14

Figure 9-29: Medium acceleration diesel drive-by without mannequin 5........................ 15

Figure 9-30: Medium acceleration diesel drive-by without mannequin 6......................... 15

Figure 9-31: Cruising diesel drive-by without mannequin 1............................................

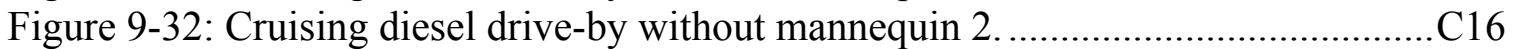

Figure 9-33: Cruising diesel drive-by without mannequin 3........................................ 17

Figure 9-34: Cruising diesel drive-by without mannequin 4........................................... 17

Figure 9-35: Cruising diesel drive-by without mannequin 5........................................... 18

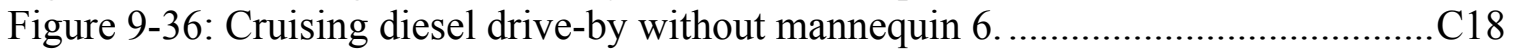

Figure 9-37: Hard acceleration diesel drive-by with baby 1 ............................................ 19

Figure 9-38: Hard acceleration diesel drive-by with baby 2......................................... 19

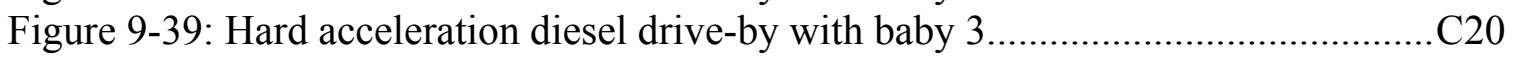

Figure 9-40: Hard acceleration diesel drive-by with baby 4 .............................................. 20

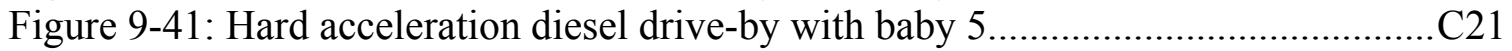

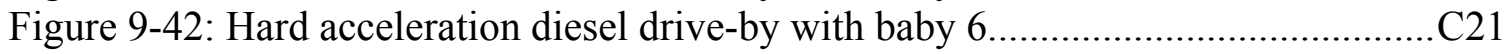

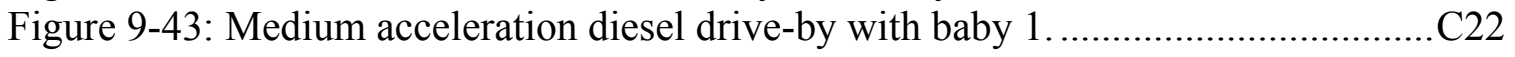

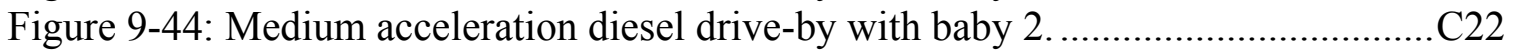

Figure 9-45: Medium acceleration diesel drive-by with baby 3 ........................................... 23

Figure 9-46: Medium acceleration diesel drive-by with baby 4 .............................................. 23

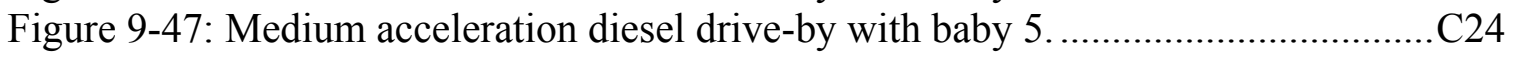

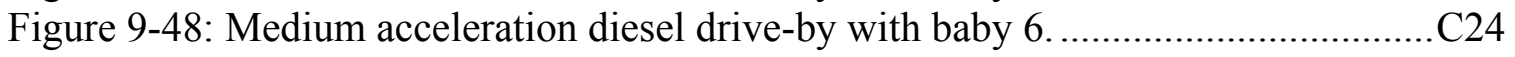

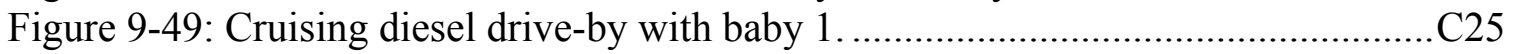

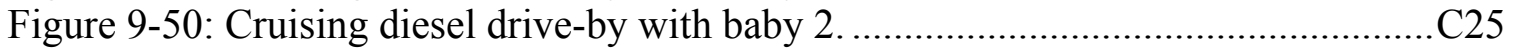

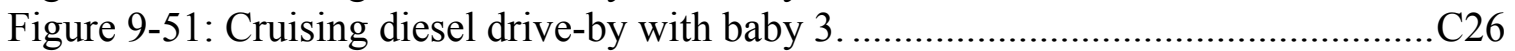




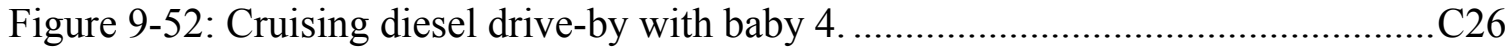

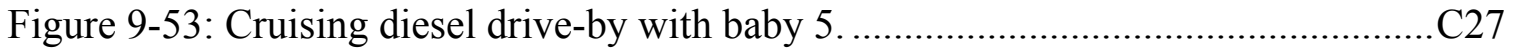

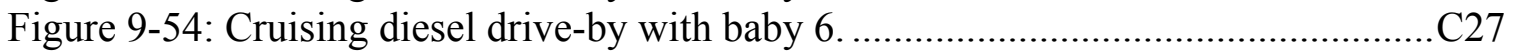

Figure 9-55: Hard acceleration gasoline drive-by with mannequin 1.................................. 28

Figure 9-56: Hard acceleration gasoline drive-by with mannequin 2............................... 28

Figure 9-57: Hard acceleration gasoline drive-by with mannequin 3............................... 29

Figure 9-58: Hard acceleration gasoline drive-by with mannequin 4....................................... 29

Figure 9-59: Hard acceleration gasoline drive-by with mannequin 5.............................. 30

Figure 9-60: Hard acceleration gasoline drive-by with mannequin 6............................. 30

Figure 9-61: Medium acceleration gasoline drive-by with mannequin 1....................... 31

Figure 9-62: Medium acceleration gasoline drive-by with mannequin 2........................ 31

Figure 9-63: Medium acceleration gasoline drive-by with mannequin 3........................ 32

Figure 9-64: Medium acceleration gasoline drive-by with mannequin 4......................... 32

Figure 9-65: Medium acceleration gasoline drive-by with mannequin 5......................... 33

Figure 9-66: Medium acceleration gasoline drive-by with mannequin 6.......................... 33

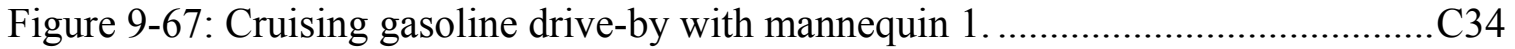

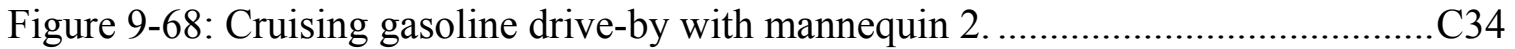

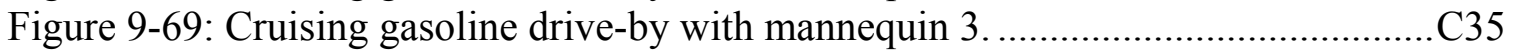

Figure 9-70: Cruising gasoline drive-by with mannequin 4 .............................................. 35

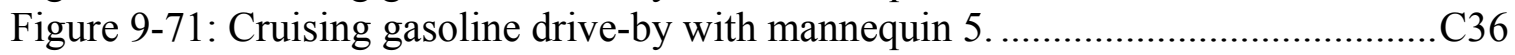

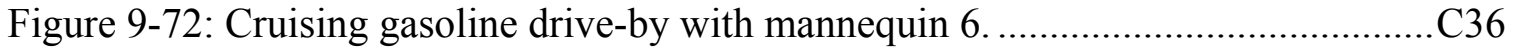

Figure 9-73: Hard acceleration gasoline drive-by without mannequin 1......................37

Figure 9-74: Hard acceleration gasoline drive-by without mannequin 2. ...................... 37

Figure 9-75: Hard acceleration gasoline drive-by without mannequin 3 ........................ 38

Figure 9-76: Hard acceleration gasoline drive-by without mannequin 4. .......................... 38

Figure 9-77: Hard acceleration gasoline drive-by without mannequin 5....................... 39

Figure 9-78: Hard acceleration gasoline drive-by without mannequin 6........................ 39

Figure 9-79: Medium acceleration gasoline drive-by without mannequin 1.................C40

Figure 9-80: Medium acceleration gasoline drive-by without mannequin 2..................... 40

Figure 9-81: Medium acceleration gasoline drive-by without mannequin 3.................... 41

Figure 9-82: Medium acceleration gasoline drive-by without mannequin 4.................C41

Figure 9-83: Medium acceleration gasoline drive-by without mannequin 5..................C42

Figure 9-84: Medium acceleration gasoline drive-by without mannequin 6..................C42

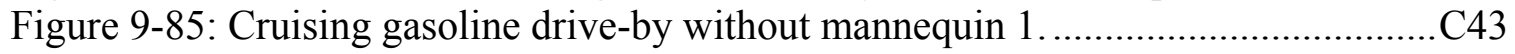

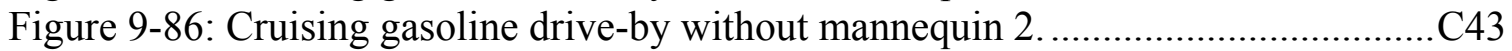

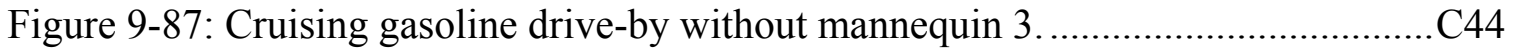

Figure 9-88: Cruising gasoline drive-by without mannequin 4.......................................... 44

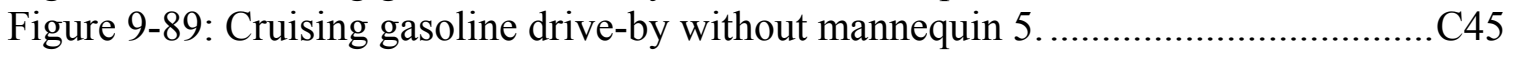

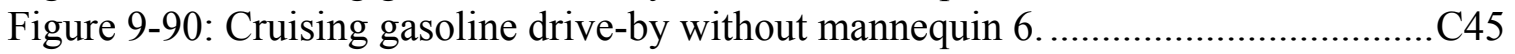

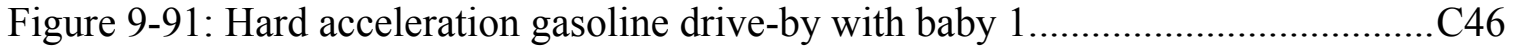

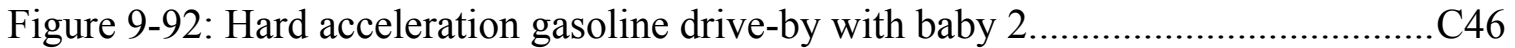

Figure 9-93: Hard acceleration gasoline drive-by with baby 3......................................... 47

Figure 9-94: Hard acceleration gasoline drive-by with baby 4............................................ 47

Figure 9-95: Hard acceleration gasoline drive-by with baby 5........................................... 48

Figure 9-96: Hard acceleration gasoline drive-by with baby $6 \ldots \ldots \ldots \ldots \ldots \ldots \ldots \ldots \ldots \ldots \ldots \ldots \ldots \ldots . . . \mathrm{C} 48$

Figure 9-97: Medium acceleration gasoline drive-by with baby 1................................ 49 


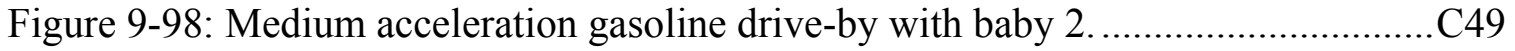

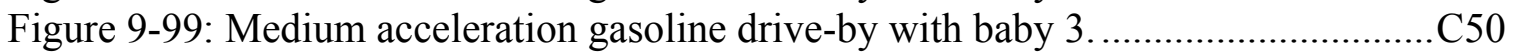

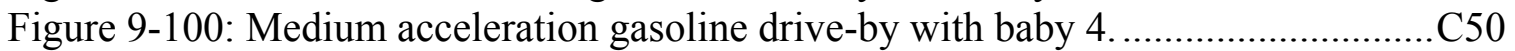

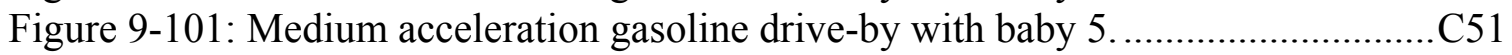

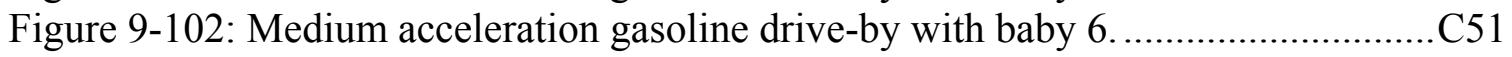

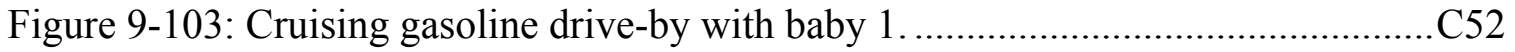

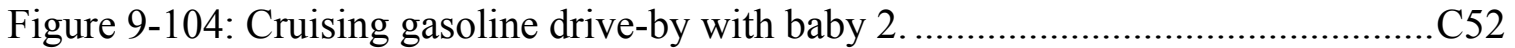

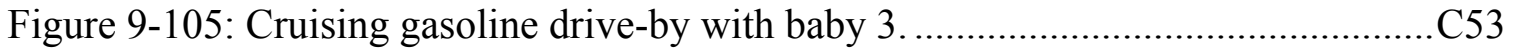

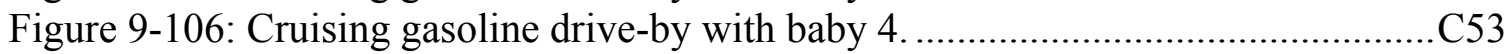

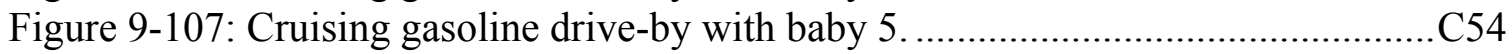

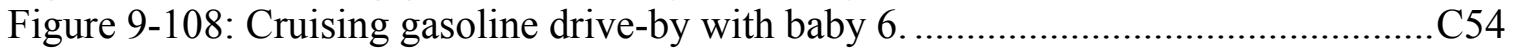

Figure 10-1: Lognormal particle size distribution for hard acceleration diesel vehicle tests

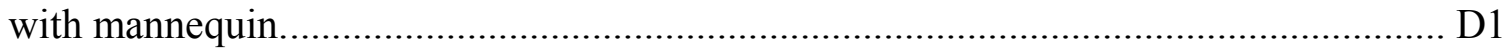

Figure 10-2: Lognormal particle size distribution for hard acceleration diesel vehicle tests with stroller. D1

Figure 10-3: Lognormal particle size distribution for hard acceleration diesel vehicle tests

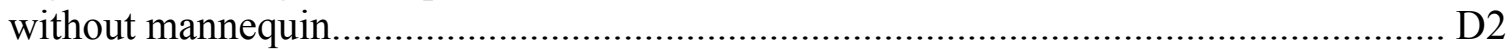

Figure 10-4: Lognormal particle size distribution for medium acceleration diesel vehicle tests with mannequin. D2

Figure 10-5: Lognormal particle size distribution for medium acceleration diesel vehicle tests with stroller. D3

Figure 10-6: Lognormal particle size distribution for medium acceleration diesel vehicle tests without mannequin. D3 Figure 10-7: Lognormal particle size distribution for cruising diesel vehicle tests with mannequin. D4 Figure 10-8: Lognormal particle size distribution for cruising diesel vehicle tests with stroller.

Figure 10-9: Lognormal particle size distribution for cruising diesel vehicle tests without mannequin... D5 Figure 10-10: Lognormal particle size distribution for hard acceleration gasoline vehicle tests with mannequin. D5 Figure 10-11: Lognormal particle size distribution for hard acceleration gasoline vehicle tests with stroller. D6 Figure 10-12: Lognormal particle size distribution for hard acceleration gasoline vehicle tests without mannequin. D6 Figure 10-13: Lognormal particle size distribution for medium acceleration gasoline vehicle tests with mannequin. D7 Figure 10-14: Lognormal particle size distribution for medium acceleration gasoline vehicle tests with stroller. D7 Figure 10-15: Lognormal particle size distribution for medium acceleration gasoline

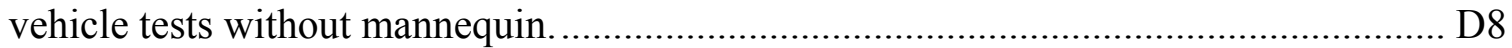
Figure 10-16: Lognormal particle size distribution for cruising gasoline vehicle tests with mannequin. D8 Figure 10-17: Lognormal particle size distribution for cruising gasoline vehicle tests with stroller. D9 
Figure 10-18: Lognormal particle size distribution for cruising gasoline vehicle tests without mannequin ................................................................................................. D9 


\section{Nomenclature and Abbreviations}

\begin{tabular}{|c|c|}
\hline ACEA & European Automobile Manufacturers' Association \\
\hline $\mathrm{cc}$ & Cubic Centimeter \\
\hline $\mathrm{CO}$ & Carbon Monoxide \\
\hline $\mathrm{CO}_{2}$ & Carbon Dioxide \\
\hline CARB & California Air Resources Board \\
\hline $\mathrm{CRC}$ & Coordinating Research Council \\
\hline DE & Diesel Exhaust \\
\hline DEARS & Detroit Exposure and Aerosol Study \\
\hline $\mathrm{DOC}$ & Diesel Oxidation Catalyst \\
\hline DPF & Diesel Particulate Filter \\
\hline DPM & Diesel Particulate Matter \\
\hline $\mathrm{EC}$ & Elemental Carbon \\
\hline ECU & Engine Control Unit \\
\hline EU & European Union \\
\hline $\mathrm{HC}$ & Hydrocarbon \\
\hline HEI & Health Effects Institute \\
\hline $\mathrm{hp}$ & Horsepower \\
\hline $\mathrm{Hz}$ & Hertz \\
\hline IARC & International Agency for Research on Cancer \\
\hline $\mathrm{L}$ & Liters \\
\hline $\mathrm{m}$ & Meters \\
\hline MPH & Miles Per Hour \\
\hline MSHA & Mine Safety and Health Administration \\
\hline NAAQS & National Ambient Air Quality Standards \\
\hline NDIR & Non-Dispersive Infrared \\
\hline $\mathrm{NIOSH}$ & National Institute for Occupational Safety and Health \\
\hline NIST & National Institute of Standards and Technology \\
\hline NOx & Oxides of Nitrogen \\
\hline OSHA & Occupational Safety and Health Administration \\
\hline PAH & Polycyclic Aromatic Hydrocarbon \\
\hline PEL & Permissible Exposure Limit \\
\hline PM & Particulate Matter \\
\hline PPM & Parts Per Million \\
\hline SOF & Soluble Organic Fraction \\
\hline SOL & Solid Fraction \\
\hline $\mathrm{SOx}$ & Oxides of Sulfur \\
\hline $\mathrm{SO}_{4}$ & Sulfate \\
\hline $\mathrm{TC}$ & Total Carbon \\
\hline TEM & Transmission Electron Microscope \\
\hline TPM & Total Particulate Matter \\
\hline UFP & Ultra Fine Particles \\
\hline US & United States \\
\hline EPA & Environmental Protection Agency \\
\hline VBA & Visual Basic for Applications \\
\hline VOF & Volatile Organic Fraction \\
\hline WHO & World Health Organization \\
\hline WVU & West Virginia University \\
\hline
\end{tabular}




\section{Introduction}

Particulate matter (PM) is a nonspecific term used to describe material suspended in the atmosphere in the form of small particles or liquid droplets. When inhaled into a human's respiratory system, the smallest of these particles, ultrafine particles (UFP), can deposit deep inside the lung. Those that are only several nanometers in aerodynamic diameter can even penetrate cellular boundary membranes to deposit in the blood stream [1]. There is extensive literature that supports the relationship between particulate matter (PM) and adverse human health effects [2-30]. Chronic and acute exposure to PM has been associated with lung cancer, increased morbidity leading to hospitalization and premature mortality, as well as respiratory inflammation, asthmatic-like symptoms, and irritation. There is also concern regarding PM pollution affecting visibility in cities and national parks as well as global climate forcing [30].

Consequently, the United States Environmental Protection Agency (EPA), which is an environmental regulatory body within the US government, and the Mine Safety and Health Administration (MSHA) set standards to regulate the allowable level of ambient particulate matter and limit the maximum concentration to which persons can be exposed in an occupational mine setting, respectively. In the US, airborne particulate matter less than 2.5 microns in size $\left(\mathrm{PM}_{2.5}\right)$ is required to be at or below $35 \mu \mathrm{g} / \mathrm{m}^{3}$ averaged over a 24 hour period and at or below an annual arithmetic mean of $15 \mu \mathrm{g} / \mathrm{m}^{3}$ [31]. However, those air quality standards address exposures averaged over large spatial (greater than 100 meters) and time (24 hours) scales. In addition, the MSHA set an exposure limit on diesel particulate matter within mines of $160 \mu \mathrm{g} / \mathrm{m}^{3}$ averaged over an 8 hour period, effective January 2006 [32]. Because it is estimated that $35 \%$ of ambient $\mathrm{PM}_{2.5}$ typically is contributed by mobile sources [33], there has been interest in the possibility of health effects due to elevated exposures near roadways [34,35]. This study is concerned with exposures that are even closer to the vehicle exhaust than the distances termed "near roadway" in the literature.

While most automobile exhausts are directed to the rear of the vehicle, many vehicles around the world, especially pickup trucks, employ tailpipes that direct exhaust towards the passenger side of the vehicle. Since right side tailpipes direct emissions towards sidewalks and roadsides in countries where vehicles drive on the right side of the 
road, there is concern regarding adults, children, and infants in strollers on sidewalks and near roadways being exposed to hazardous exhaust constituents at levels greater than typical "near roadway" levels. Additionally, there is concern regarding occupational exposures to persons who are required to be near roadways or diesel vehicles for long periods of time.

When exhaust is emitted from a diesel vehicle, it can be characterized as a plume of evolving particles and gaseous material. It is expected that within this plume particle concentrations may substantially exceed regulations for brief periods. Wind tunnel studies [36,37] show that there may not be any appreciable evolution of particle size within a plume and that dilution ratios can range from 75 to 125 at a distance of 8.5 meters downstream of the tailpipe. In contrast to these findings, a vehicle chase study [38] observed actual dilution ratios as large as 1,000:1 in two seconds. However, the chase study was conducted at freeway speeds of 40 to $55 \mathrm{mph}$, which is more than double the local street speeds (20 to $25 \mathrm{mph}$ ) tested in this study. Therefore, it is reasonable to consider the possibility that dilution rates would be far lower at local street speeds for two reasons: (i) the travel time to a sidewalk is very short, providing little time to dilute appreciably, and (ii) the turbulence imparted to surrounding air by the vehicle would be much lower. Hence, it is plausible that pedestrians may be exposed to particulate matter concentrations significantly higher than regulations allow, though perhaps for brief periods as each vehicle passes or idles at the sidewalk.

Although there is a limited amount of information regarding acute and short-term (e.g., less than 8 hours) exposures to diesel exhaust (DE), there is strong evidence from human and animal studies that exposures to low concentrations of diesel exhaust (300 $\mu \mathrm{g} / \mathrm{m}^{3}$ ) can cause pathophysiological symptoms, such as particle accumulation in the lungs; acute eye, nasal and throat irritation; neurophysiological symptoms such as lightheadedness and nausea; and respiratory symptoms, including cough and phlegm [30]. Since data from dilution tunnel measurements [39-41] can not accurately simulate this type of human exposure, drive-by experiments are necessary.

The objective of this study was to quantify the short, local exposures that may be experienced by pedestrians in the immediate vicinity of a vehicle's tailpipe during a vehicle drive-by incident. This objective was accomplished by quantifying number and 
mass concentration, observing particle size distributions, comparing exposures from diesel and gasoline fueled vehicles, and comparing different exposure scenarios. Additionally, correlations between exposure and specific vehicle engine operating conditions were developed. The concentrations of particulate matter that reached a "pedestrian's" mouth were quantified and compared to human dose studies. Furthermore, short-term exposure was determined and the mass of particulate matter potentially inhaled daily given a maximum number of drive-by incidents and the mass potentially inhaled per breath were quantified. The vehicles studied here were a diesel-fueled Dodge Ram 2500 pickup truck and a gasoline-fueled Dodge Ram 1500 pickup truck, which both have original tailpipes that discharge directly toward the sidewalk. Multiple exposure scenarios, including a simulated adult or a child in a stroller beside the road, were examined for different vehicle operating conditions during the "drive-by."

\section{Literature Review}

\subsection{Particulate Matter}

Diesel powered vehicles have historically been associated with a smoke-like, black exhaust plume which is caused by PM, possibly the most visibly distinctive of combustion engine emissions. Although it is much less noticeable, gasoline powered vehicles produce PM as well, though the particles are typically fewer and smaller. PM is a very complex emission which consists of many components. Despite a considerable amount of research, PM formation in the engine cylinder, its physical and chemical properties, and human health effects associated with exposure to it are not fully understood. Nevertheless, enough is known to determine that PM is an emission produced by combustion engines capable of harming humans. Consequently, particulate expulsion is subject to emission regulations worldwide and, along with oxides of nitrogen (NOx), have become the focus for emission control technology and research [42].

PM is the term used to refer to the particles present in combustion engine exhaust and is defined as any matter in the exhaust of an internal combustion engine that can be trapped on a sampling filter medium at $125^{\circ} \mathrm{F}\left(52^{\circ} \mathrm{C}\right)$ or less [43]. PM is typically composed of highly agglomerated, solid carbonaceous material, ash, and adsorbed 
organic compounds as well as small amounts of sulfates, nitrates, metals, and other trace elements [40]. While PM is a major component of engine exhaust, as described by the EPA, engine exhaust is a complex mixture of thousands of constituents in either gas or particle form [30]. The gaseous constituents found in engine exhaust include carbon monoxide $(\mathrm{CO})$, carbon dioxide $\left(\mathrm{CO}_{2}\right)$, $\mathrm{NOx}$, oxides of sulfur ( $\left.\mathrm{SOx}\right)$, and numerous lowmolecular-weight hydrocarbons (HC) [30]. Typically, the particle size distribution and composition are of most interest.

\subsubsection{Particle Size Distribution}

According to the Air Quality Criteria document for PM prepared by the US EPA [30], "particle size, as indexed by one of the "equivalent" diameters, is an important parameter in determining the properties, effects, and fate of atmospheric particles". The Stokes and aerodynamic diameters are strongly related to the deposition rates of particles and, correspondingly, the particles' residence times in the atmosphere [30]. Particle deposition in lungs is also a function of particle size. Even visibility and the climate are affected by atmospheric particle size distribution [30]. These arguments show that the particle size distribution is important because many effects caused by PM are influenced by particle size.

Many authors categorize ambient PM mass based on the aerodynamic diameter of the particles, which is defined as the diameter of an equivalent spherical particle of unit density with the same settling velocity in air as the measured particle. While aerodynamic diameter is widely used, there are many other equivalent diameters used to characterize particle size, such as optical diameter and electrical mobility diameter, which are used to determine particle count [30]. Electrical mobility takes into account the movement of a charged particle in an electric field as well as the settling velocity considered for aerodynamic mobility. $\mathrm{PM}_{10}$ is the term used for particulates of aerodynamic diameter less than $10 \mu \mathrm{m}$, fine particles have diameters below $2.5 \mu \mathrm{m}$, ultrafine particles have diameters less than $0.1 \mu \mathrm{m}$ or $100 \mathrm{~nm}$, and nanoparticles are characterized by diameters less than $50 \mathrm{~nm}$ [40]. "Typical engine exhaust mass and number weighted size distributions along with alveolar deposition fraction" are shown in Figure 2-1 [40]. It can 
be seen that nearly all of the particles have diameters much less than $1 \mu \mathrm{m}$. Therefore, PM represents a mixture of nanoparticles, ultrafine particles, and fine particles.

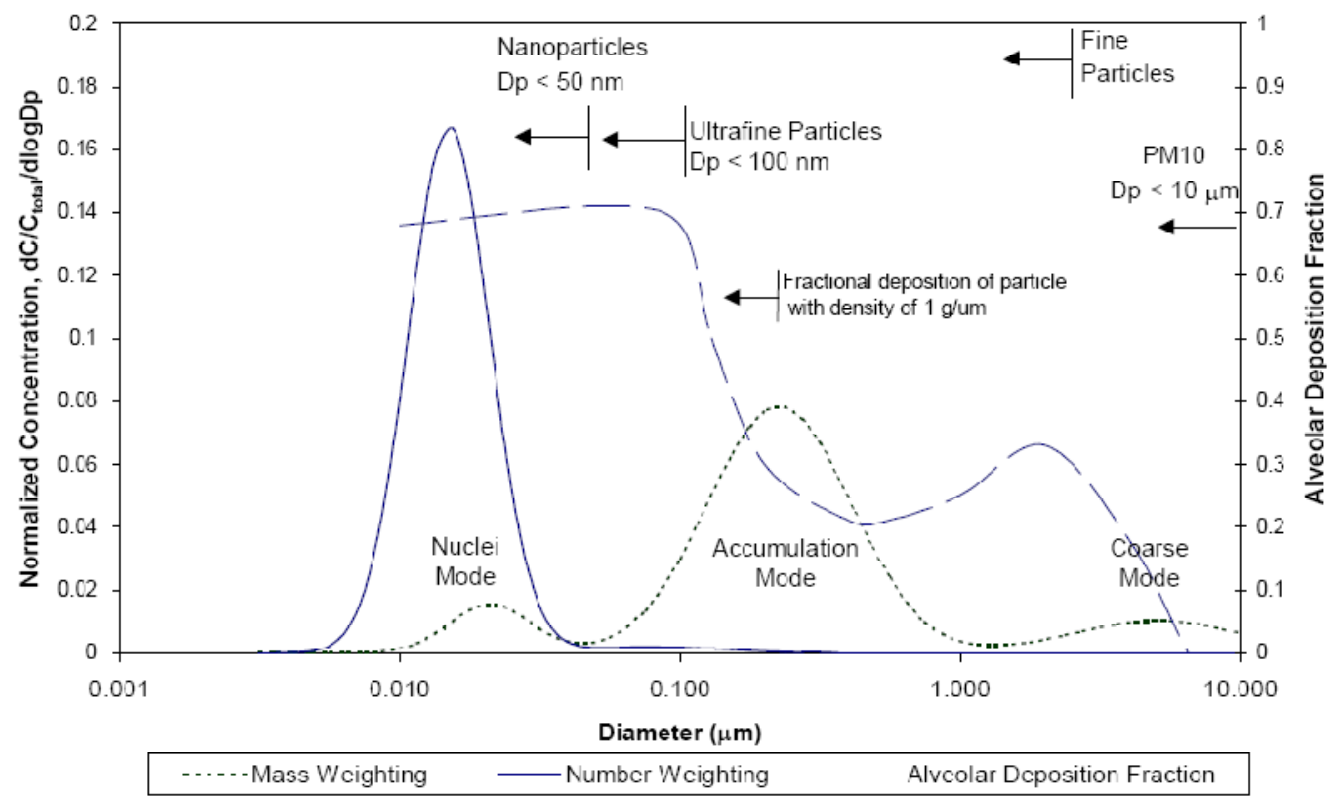

Figure 2-1: “Typical engine exhaust mass and number weighted size distribution shown with alveolar deposition fraction" [40].

The size distributions of PM typically have a well recognized lognormal, trimodal distribution which corresponds to the particle nucleation, agglomeration, and reentrainment mechanisms associated with nuclei, accumulation, and coarse mode particles, respectively [40]. Nevertheless, bimodal distributions are more commonly observed in actual vehicle exhaust measurements [38]. Usually, these modes have well defined peaks on a typical size distribution plot. However, this depends greatly on whether the plot is using particle mass or particle number weighting. If the distribution is plotted via particle number, the peak typically occurs around the nuclei mode while the peak is generally found near the accumulation mode when using particle mass.

The on-road nuclei mode has been postulated to account for the particle size range from 3 to $30 \mathrm{~nm}$ based on on-road diesel particle size research conducted in the 1990s $[38,44]$. This conclusion implies that the nuclei mode lies entirely within the nanoparticle range. Typically, the nuclei particles account for $0.1-10 \%$ of the PM mass and often more than $90 \%$ of the total particulate count [38]. Additionally, nuclei mode particles have been found to consist of little solid material. Rather, they are mostly composed of volatile condensates such as hydrocarbons and sulfuric acid [38]. The accumulation mode is 
believed to consist of particles with aerodynamic diameters in the range of 30-500 nm [38]. Thus, this mode extends from the upper half of the nanoparticle range through the ultrafine and fine particle ranges. Accumulation mode particles are typically composed of carbon agglomerate solids mixed with condensates and adsorbed material. The accumulation mode usually accounts for about $10 \%$ of the particle number and $80-90 \%$ of the PM mass [38]. The coarse mode includes particles with diameters above 1,000 nm and usually accounts for $5-20 \%$ of the total PM mass [38]. However, they add almost nothing to the total particle number. Coarse mode particles are not formed in the combustion process. Rather, they are produced through deposition and re-entrainment of particulate material from walls of the engine cylinder and exhaust system.

Because it is believed that fine and ultrafine particles are more toxic and can penetrate deeper into the lung than larger particles [30], internal combustion engine particle size distributions have been getting more attention since the mid-1990s. As regulations have decreased the allowable particulate mass emitted from engines, diesel emission control strategies using engine design or aftertreatment technologies are being reviewed and inspected to determine their effectiveness in the control and suppression of the smallest diesel particles and particle number emissions. However, note that the EPA has not yet set any regulations on particle number emissions. The European Union (EU), on the other hand, has passed new legislation which will regulate particle number emissions of certain diesel vehicle classes in addition to the already stringent particle mass standards [45].

A study by the Health Effects Institute (HEI) showed the possibility that those emission control strategies may actually increase fine and ultrafine particle number emissions [46]. Thus, in order to evaluate various control technologies, a definition and correct measurement techniques of the smallest particles must be agreed upon. This is because the measurement of particle sizes and numbers is much more sensitive to the techniques utilized than is the determination of particulate mass emissions. Dilution and sampling methods are important parameters that must also be considered to guarantee accurate and repeatable results. However, standardized measuring methods have not yet been developed in the US. The new legislation passed by the EU is supposed to introduce 
a new method for measuring PM mass which will be standardized and will account for differences in old techniques such that comparisons of measurements are valid [45].

As just discussed, many current emission control strategies are being reviewed and examined because their success in reducing particle number emissions is believed to be poor. This is because many current emission control strategies focus on reduction of particle mass rather than particle number. Consequently, newer model engines are believed to have higher numbers of fine and ultrafine particles than older model engines. The HEI, for example, conducted one of the first studies with this consideration in mind that compared the particulate emissions from a 1988 and a 1991 diesel Cummins engines, and the results suggested newer model engines may have higher fine and ultrafine particle number emissions than older model engines [47]. Data from that study showed that there was a 90\% reduction in PM mass in the accumulation mode from the 1991 engine relative to the 1988 engine, but that the nuclei mode had increased by as much as $40 \%$ of the total particle volume. However, note that the "new" vehicle in that study no longer represents "new" vehicles especially because the vehicle was not equipped with a diesel particulate filter (DPF), a diesel oxidation catalyst (DOC), or any other new emission reduction equipment.

In addition to the HEI study, a number of other studies have been conducted which compare newer and older model year engines. However, the observed emission differences were much less dramatic than the HEI study. For example, the VERT study compared two Liebherr diesel engines and found that particle number emissions increased by 15 to $50 \%$ over most operating conditions [48]. Another study, funded by the Coordinating Research Council (CRC), examined particle number emissions from heavy duty engines as part of a comprehensive diesel aerosol sampling study [38]. For this study, particle emissions data were collected from several vehicles with 1989 and 1999 model year heavy duty diesel engines via highway chase experiments using a mobile emissions laboratory. The nanoparticle emissions from those vehicles were found to be considerably less than that of the engine used in the HEI study. Several years before that study, chase experiments were also conducted on a vehicle with a 1979 model year diesel engine for a different CRC project known as (AP2) [49]. The particle number data from that study revealed particle number emissions that were higher than those in the HEI 
study as well as the more recent chase experiments conducted with newer model year engines. These results imply that nanoparticle emissions have been around for a long time and that they are not necessarily a new occurrence caused by new engine technologies.

Other studies have reported decreased particle number emissions with more advanced engine technology in Euro I, Euro II, and Euro III engines tested over regulatory emission test cycles [50,51]. Additional studies funded by ACEA found that the size distributions of particle number emissions were similar between older and newer diesel passenger cars and that particulate mass could be correlated with particle number [52]. While each of the studies mentioned have very different results showing that particle number emissions vary greatly depending on engine technologies, comparisons of data from those studies are invalid because no standard measurement method exists.

\subsubsection{Composition of Particulate Matter}

PM is a complex mixture composed of particles in both the solid and liquid form. Typically, it is divided into three main categories or fractions as well as several subcategories [42]. The first fraction is known as the solid fraction (SOL) which is a combination of elemental carbon and ash. Another fraction, referred to as the soluble organic fraction (SOF) or organic carbon, comes from organic material derived from engine lubricating oil and from fuel. The final fraction, composed of sulfate particles $\left(\mathrm{SO}_{4}\right)$, is a mixture of sulfuric acid and water. Together these three fractions make up the total particulate matter (TPM).

Elemental carbon, often identified as the "inorganic carbon", is the main constituent of the SOL of particulates and is the cause of the black smoke traditionally associated with diesel vehicles [42]. Because it is not chemically bound to other elements, this carbon is finely dispersed in the form of "soot" or "carbon black" which is the substance responsible for the black appearance of the smoke [42]. This carbonaceous PM fraction results from the heterogeneous combustion process in diesel engines, where solid particle precursors are formed in both the diffusion and premixed flames [42]. These particle precursors or primary particles begin to agglomerate in the cylinder and continue to agglomerate through the exhaust system and after expulsion into the atmosphere. For measurement purposes, the shape of these particles is desired. It has 
been shown in transmission electron microscope (TEM) pictures that nuclei particles are nearly spherical while agglomerated particles that make up the accumulation mode are not spherical. Rather, they often form extended chain-like structures [53]. This knowledge of the particles' shapes makes it difficult to size PM because different measurement principles require the use of various types of equivalent particle diameters to express particle sizes.

Although elemental carbon is the primary component of the SOL of particulate matter, metallic ash also is an important component. As the production of newer engines focuses on reducing carbon particulates, non-carbon solid particulate emissions, such as ash, are increasing in importance. A study concerning the emissions from US post-1994 heavy duty engines determined that the PM from these engines may contain up to $10 \%$ ash and in some cases even more [54]. As particulate regulations on newer engines become more stringent, this percentage may increase further. DPF material developers are the reason ash receives a lot of attention because the filter materials must be resistant to corrosion by ash compound [55]. Generally, ash from engine exhaust consists of a mixture of sulfates, phosphates, or oxides of calcium, zinc, magnesium, and other metals. These constituents are produced when additives in the engine lubricating oil are burned in the combustion chamber, metal oxide impurities from engine wear are carried into the cylinder by the lube oil, and iron oxides resulting from corrosion of exhaust system components are carried into the exhaust stream [55].

Solid phase, carbon material (SOL) is the chief constituent of particles leaving the engine mostly in the form of individual nuclei mode and agglomerated carbon particles formed in the engine cylinder [42]. Other solid material in diesel exhaust comes from metal ash compounds derived from lubricating oil additives in addition to engine wear. These ash nuclei, believed to nucleate during the expansion stroke in the engine cylinder, can agglomerate to form accumulation mode particles [42]. Depending on the temperature, the particles leaving the engine cylinder undergo partial oxidation and further agglomeration while in transport through the exhaust system [42]. During transport, thermophoretic forces (i.e., temperature difference driven mass transfer) cause some particles to deposit on the exhaust pipe walls. Additionally, PM precursors such as 
hydrocarbons, sulfur oxides, and water are present as gases or vapors in the hot diesel exhaust [42].

The SOF of particulates consists of hydrocarbons adsorbed onto the surface of carbon particles and in the form of fine droplets. The term soluble in the name of this fraction originated from the analytical technique of using solvents to isolate the organic fraction of particulates [42]. However, this fraction is occasionally referred to as the volatile organic fraction (VOF) which is typically measured with vacuum evaporation. In some instances, though, the evaporated fraction may contain water-soluble material such as sulfates. It should be mentioned that the SOF begins as mostly vapor in the hot engine exhaust and only becomes liquid after cooling to below $52^{\circ} \mathrm{C}$ usually during dilution [42]. This is important because the changes that occur greatly affect aftertreatment devices.

Because the percentage of SOF in PM may differ considerably between engines, terms were developed to imply high or low SOF content (high or low organic carbon content). Particulates with high SOF content are referred to as "wet" particulates while those with low SOF content are referred to as "dry" particulates [42]. The SOF may compose over $50 \%$ of the total PM for wet particulates while in dry particulates the percentage may be $10 \%$ or lower [42]. In contrast, the percentage of PM not composed of SOF is usually composed of elemental carbon. Thus, elemental carbon can be a good indicator of PM exposure in "dry" PM. Typically, light engine loads tend to produce the highest content of SOF because exhaust temperatures are low [42]. The SOF is mostly comprised of lube oil derived hydrocarbons with diesel fuel hydrocarbons having higher boiling points making up a very small portion. However, the percentages of unburned fuel and lubrication oil vary widely with test cycle and engine [56,57]. A study comparing chromatograms of diesel SOF with diesel fuel and lubrication oil showed that the chromatogram of diesel SOF was very similar to that of the lube oil [53].

Polynuclear (or polycyclic) aromatic hydrocarbons (PAHs) and nitro-PAHs discharged with DE are mostly found in the SOF [42]. PAHs are aromatic hydrocarbons with two or more benzene rings joined in various, clustered forms. They are of special concern because they have mutagenic and sometimes carcinogenic characteristics. Consequently, the US EPA has defined them as a class of air toxic compounds which 
includes seven PAHs identified as probable human carcinogens [42]. In addition to PAHs, dioxins are found in the SOF of particulates. Dioxin is a generic term used to refer to a specific group of chlorinated hydrocarbon compounds that are characterized by extremely high toxicity, probable carcinogenicity, and resistance to biological breakdown [42]. The US EPA reviewed existing literature concerning dioxins and provided estimates for dioxin emission factors from internal combustion engines in a draft dioxin health assessment document [58].

Sulfate particles consist mostly of hydrated sulfuric acid and, consequently, are mainly liquid. Sulfate particulates form by means of an interaction between sulfuric acid and water molecules. The process is theoretically modeled as heteromolecular nucleation, which states that both sulfuric acid and water vapor can produce particles even when undersaturated [47]. Nucleation begins with small molecular bunches that grow into more stable nuclei particles. When the molar ratio of water to sulfuric acid reaches $8: 3$, most stable sulfate particulates are produced.

\subsection{Regulations and Standards}

DE is known to be harmful to human health. However, some of the substances found in DE are benign while others cause adverse health effects. As such, the US EPA has identified several components of DE as pollutants. The components of DE that are presently regulated are $\mathrm{PM}, \mathrm{NOx}, \mathrm{HC}$, and $\mathrm{CO}$ [59]. Because most of the sources for these pollutants are mobile, there are two ways which the EPA monitors and regulates these emissions. The first way is through "tailpipe" emission standards. These standards are based on integrated cycle specific emissions discharged from an engine. To ensure that a vehicle abides by these standards, vehicles and engines must meet certification requirements. Another way the EPA monitors and regulates emissions is via ambient air quality standards. Cities, towns and areas all are required to meet specific air quality standards which the EPA sets. Adherence to these standards is monitored via a network of ambient emission monitoring stations located all over the country.

A vehicle or engine is emission certified by measuring the emissions from the vehicle or engine over a vehicle or engine test cycle and comparing the results to the appropriate standard. This is accomplished by attaching the vehicle or the engine to a 
chassis or engine dynamometer, respectively, and operating the vehicle or engine through a test cycle. Test cycles are designed to create repeatable emissions measurement conditions while simultaneously attempting to represent real world driving conditions for a given application. Additionally, the methods used to measure most emissions are also regulated by the standards such that comparisons between measurements taken at different facilities are valid. The procedure in addition to the specifications required by facilities, measurement equipment, and measurement methods are described in detail in the Code of Federal Regulations Title 40 Part 86 and 1065 [60]. In the US, emission standards for new engines and/or vehicles are separated into different categories; cars and light trucks are separated into Tier 1, Tier 2, and California, heavy duty trucks and buses are lumped together into one category, mobile non-road diesels have a single category, railway locomotives have a single category, marine engines have a single category, spark ignited engines are split into two categories, small and large spark ignited engines, and stationary diesel engines have a separate category [32]. However, it must be noted that in the past heavy duty trucks and buses had separate categories. In particular, the bus standards were typically more stringent.

The EPA uses a network of measurement facilities and devices to measure ambient concentrations of pollutants mentioned above at hundreds of locations across the country. The Clean Air Act, which was last amended in 1990, requires the EPA to set National Ambient Air Quality Standards (NAAQS) [61] for pollutants considered harmful to public health and the environment [31]. The Clean Air Act established two different types of standards, one to address public health, and the other to address public welfare including decreased visibility and damage to crops, vegetation, and buildings. A table showing the NAAQS for six principal pollutants also known as "criteria" pollutants can be seen below in Table 2-1. Using these measurements, the EPA can determine whether or not areas meet the NAAQS. 
Table 2-1: National ambient air quality standards [31].

\begin{tabular}{|c|c|c|c|c|}
\hline & \multicolumn{2}{|c|}{ Primary Standards } & \multicolumn{2}{|c|}{ Secondary Standards } \\
\hline Pollutant & Level & Averaging Time & Level & $\begin{array}{c}\text { Averaging } \\
\text { Time }\end{array}$ \\
\hline \multirow[t]{2}{*}{$\begin{array}{l}\text { Carbon } \\
\text { Monoxide }\end{array}$} & $\begin{array}{l}9 \mathrm{ppm} \\
\left(10 \mathrm{mg} / \mathrm{m}^{3}\right)\end{array}$ & 8 -hour 11 & \multirow{2}{*}{\multicolumn{2}{|c|}{ None }} \\
\hline & $\begin{array}{l}35 \mathrm{ppm} \\
\left(40 \mathrm{mg} / \mathrm{m}^{3}\right)\end{array}$ & 1 -hour (1) & & \\
\hline \multirow[t]{2}{*}{ Lead } & $0.15 \mu \mathrm{g} / \mathrm{m}^{3}(2)$ & $\begin{array}{l}\text { Rolling 3-Month } \\
\text { Average }\end{array}$ & \multicolumn{2}{|c|}{ Same as Primary } \\
\hline & $1.5 \mu \mathrm{g} / \mathrm{m}^{3}$ & Quarterly Average & \multicolumn{2}{|c|}{ Same as Primary } \\
\hline $\begin{array}{l}\text { Nitrogen } \\
\text { Dioxide }\end{array}$ & $\begin{array}{l}0.053 \mathrm{ppm} \\
\left(100 \mu \mathrm{g} / \mathrm{m}^{3}\right)\end{array}$ & $\begin{array}{l}\text { Annual } \\
\text { (Anithmetic Mean) }\end{array}$ & \multicolumn{2}{|c|}{ Same as Primary } \\
\hline \begin{tabular}{|l|} 
Particulate \\
Matter $\left(\mathrm{PM}_{10}\right)$
\end{tabular} & $150 \mu \mathrm{g} / \mathrm{m}^{3}$ & 24 -hour $\frac{(3)}{3}$ & \multicolumn{2}{|c|}{ Same as Primary } \\
\hline \multirow{2}{*}{$\begin{array}{l}\text { Particulate } \\
\text { Matter } \\
\left(\mathrm{PM}_{2.5}\right)\end{array}$} & $15.0 \mu \mathrm{g} / \mathrm{m}^{3}$ & $\begin{array}{l}\text { Annual } \frac{(4)}{4} \\
\text { (A-ithmetic Mean) }\end{array}$ & \multicolumn{2}{|c|}{ Same as Primary } \\
\hline & $35 \mu \mathrm{g} / \mathrm{m}^{3}$ & 24-hour (3) & \multicolumn{2}{|c|}{ Same as Primary } \\
\hline \multirow[t]{3}{*}{ Ozone } & $\begin{array}{l}0.075 \text { ppm (2008 } \\
\text { std) }\end{array}$ & 8 -hour $\frac{(6)}{2}$ & \multicolumn{2}{|c|}{ Same as Primary } \\
\hline & $\begin{array}{l}0.08 \mathrm{ppm}(1997 \\
\text { std) }\end{array}$ & 8 -hour $\frac{(7)}{2}$ & \multicolumn{2}{|c|}{ Same as Primary } \\
\hline & $0.12 \mathrm{ppm}$ & $\begin{array}{l}1 \text {-hour }(\overline{8}) \\
\text { (Applies only in } \\
\text { limited areas) }\end{array}$ & \multicolumn{2}{|c|}{ Same as Primary } \\
\hline \multirow[t]{2}{*}{$\begin{array}{l}\text { Sulfur } \\
\text { Dioxide }\end{array}$} & $0.03 \mathrm{ppm}$ & $\begin{array}{l}\text { Annual } \\
\text { (Áthmetic Mean) }\end{array}$ & \multirow[t]{2}{*}{$\begin{array}{l}0.5 \mathrm{ppm} \\
\left(1300 \mu \mathrm{g} / \mathrm{m}^{3}\right)\end{array}$} & \multirow[t]{2}{*}{ 3-hour $\frac{(1)}{2}$} \\
\hline & $0.14 \mathrm{ppm}$ & 24 -hour (1) & & \\
\hline
\end{tabular}

In addition to these standards which concern public health and welfare, there are regulations and standards that address occupational exposure to particulate matter and diesel exhaust for particular occupations. Currently, there are no legal non-mining occupational exposure limits for diesel particulate matter (DPM) in the US [32]. However, the Occupational Safety and Health Administration (OSHA), the Mine Safety and Health Administration (MSHA), and the National Institute for Occupational Safety and Health (NIOSH) have all set permissible exposure limits (PEL) on respirable dust which are applied to the particulate fraction of diesel exhaust. The OSHA's PEL for respirable dust is $5 \mathrm{mg} / \mathrm{m}^{3}$, while the NIOSH has not set a limit [8]. The MSHA, on the other hand, has a PEL for respirable dust in underground coal mines of $2 \mathrm{mg} / \mathrm{m}^{3}$ but does not have one for metal or non-metal mines [8]. The MSHA has established the only occupational exposure regulation for DPM in the US, which is $160 \mu \mathrm{g} / \mathrm{m}^{3}$ based on total carbon (TC), which includes both the elemental carbon and the organic carbon but excludes the inorganic ash and sulfates [32].

\subsection{Health Effects of Particulate Matter}

Over the years, many organizations that deal with health and safety have established evidence of a relationship between DPM and adverse health effects. In 1988, 
DE was classified as "a potential occupational carcinogen" by the NIOSH [8]. In 1989, diesel engine exhaust was considered to probably be carcinogenic to humans by the International Agency for Research on Cancer (IARC). The HEI and the World Health Organization (WHO) both found evidence showing connections between exposure to DE and lung cancer [62,63]. In 1998, the California Air Resources Board (CARB) declared DPM to be a Toxic Air Contaminant backed by a detailed investigation conducted by the California Office of Environmental Health Hazard Assessment (OEHHA) [9,10]. In 1999, the Health Effects Institute (HEI) correlated the association between the lung cancer risk and exposure to diesel emissions [11]. In 2002, the US EPA concluded that long-term exposure to DE may "pose a lung cancer hazard to humans" and short-term exposure "can cause irritation and inflammatory symptoms of a transient nature" [30].

When considering how a particular substance affects humans, both short-term and long-term exposure must be considered. As such, there have been a considerable number of scientific studies that attempted to quantify the carcinogenic and noncarcinogenic effects of exposure to DE and particulates. For example, several studies have indicated that "environmental exposure to DE may present a lung cancer hazard to humans" [30]. Based on limited human evidence and confirmatory animal evidence, the NIOSH classified DE as a potential occupational carcinogen [8]. Conclusions from this review showed that rats developed malignant and benign lung tumors due to overload after exposure to high concentrations of diesel particulates. However, the same was not true for other animals such as mice, hamsters, and monkeys. More recently, the HEI published a review of all human epidemiologic studies which stated that "long-term exposure to DE in a range of occupational settings is associated with small increases in the relative risk of lung cancer occurrence, mortality, or both" [11]. In particular, it was found that the particulate fraction of DE holds the most responsibility for its carcinogenic effects [30].

The non-cancer health effects of chronic occupational exposure to DE have been evaluated with several epidemiologic studies as well. One study examined the respiratory health status of 823 coal miners exposed to DE and 823 miners not exposed to DE [64]. It was observed that miners exposed to DE had higher occurrence rates of cough and phlegm than miners not exposed to DE. Another study reported diesel bus garage workers exposed to DE had a higher incidence of cough, phlegm and wheezing than workers not 
exposed to DE [65]. However, there have not been studies to date with scale large enough to look at consistent effects on pulmonary function with respect to chronic exposure to DE. Several studies were conducted, though, which compared the health effects of whole DE and filtered DPM [66-70]. Those studies evaluated the toxic effects of whole DE and filtered DPM on laboratory animals with the conclusion that DPM particles are the primary etiologic agents of noncancer health effects in laboratory animals. Chronic and long-term exposure to DPM is likely to pose a lung cancer hazard to humans, and it may also cause respiratory symptoms, possible neurological and behavioral effects, as well as liver effects.

In addition to chronic and long-term exposure to DE, many studies have focused on the adverse health effects associated with acute and short-term exposure to DE. Numerous studies considering effects of short-term exposure to DE on humans and animals have been carried out over the past few decades. Several studies [71-73] were conducted where human volunteers were exposed to diluted DE in an exposure chamber. Symptoms of irritation of the eyes and nose as well as an unpleasant smell were reported. Two other studies showed occupational short-term overexposure to DE could be responsible for symptoms workers developed such as persistent asthma, headache, heartburn as well as vomiting [74,75].

More recently, though, several studies have been conducted which focus on the more acute medical effects of short-term exposure to diesel exhaust. One study was conducted in which 15 healthy volunteers were exposed to diluted DE with a $\mathrm{PM}_{10}$ concentration of $300 \mu \mathrm{g} / \mathrm{m}^{3}$ for 1 hour during exercise. It was found that systemic and pulmonary inflammatory response is well-defined and clearly marked after that acute short-term exposure to DE [28]. Another study exposed 25 healthy and 15 asthmatic participants to DE with a PM concentration of $100 \mu \mathrm{g} / \mathrm{m}^{3}$ for 2 hours. A change in lung function (airway resistance) was observed in both healthy and asthmatic patients as were small changes in some markers of inflammation for the healthy participants but not the asthmatic ones [21]. A case-crossover study was conducted in which 691 patients who had myocardial infarction and survived at least 24 hours after the event were interviewed and asked to keep journals of the symptoms. The conclusion of the study was that susceptible persons may have an increased risk of myocardial infarction due to transient 
exposure to traffic [27]. In response to the case-crossover study mentioned above, the vascular and endothelial function effects in humans due to DE inhalation were investigated in another study. After exposing 30 healthy men to diluted DE with a PM concentration of $300 \mu \mathrm{g} / \mathrm{m}^{3}$ for 1 hour during intermittent exercise, it was concluded that inhalation of DE impairs the regulation of vascular tone and endogenous fibrinolysis [22].

In a very similar study, [20] men with prior myocardial infarction were exposed to the same concentration of $\mathrm{DE}$ as in the previous study for 1 hour during rest and moderate exercise. Conclusions of this study include the promotion of myocardial ischemia and inhibited fibrinolytic capacity in men with coronary heart disease due to exposure to diluted DE [23]. The findings also point to mechanisms which may explain the association between air pollution from combusted sources and unfavorable cardiovascular events. In a study which added to a previous study completed by the same researchers, 15 healthy men were exposed to diluted DE with a PM concentration of 300 $\mu \mathrm{g} / \mathrm{m}^{3}$ for 1 hour and observed effects 24 hours after the exposure. It was found that there is a "selective and persistent impairment of endothelium-dependent vasodilation which occurs in the presence of systemic inflammation" [29]. A different study was conducted in which 10 human volunteers were exposed to diluted DE with a PM concentration of $300 \mu \mathrm{g} / \mathrm{m}^{3}$ for 1 hour. It was found that DE exposure has a functional effect in the human brain, indicated by a cortical stress response [20]. In two studies by the same researchers, volunteers were exposed to 100 and $200 \mu \mathrm{g} / \mathrm{m}^{3}$ of fine PM for 2 hours. One study found no consistent effect of PM exposure on the autonomous control of the heart while the other study found acute endothelial response and vasoconstriction of a conductance artery to be associated with short-term exposure to DE [24,26].

The EPA produced a comprehensive document, the Air Quality Criteria for PM, which gives a full review of PM and how it relates to adverse health effects in humans and animals in addition to its effects on the environment [30]. Studies of short-term effects of DE on laboratory animals showed that overload of DPM particles can be lethal. However, little evidence exists which shows that short-term exposure to DE at lower levels can still impair lung function [30]. In summary, the adverse health effects of acute and short-term exposure to DE can be summarized as inducing irritations, inflammatory 
responses in the airways and the lungs typical of asthma, possible immunological and allergenic effects, and acute cardiopulmonary effects.

In order to determine how ambient particulate levels affect whole populations, many studies have been conducted all over the world which address the mortality rates at low exposure levels to daily variations in PM concentration. The risk estimated from 18 studies conducted in 18 different locations was summarized by WHO [76]. In those studies, particles near the size range of $\mathrm{PM}_{10}$ were actually measured and correlated to mortality data. The observed relationship was that for every $10 \mu \mathrm{g} / \mathrm{m}^{3}$ increase in 24-hour average $\mathrm{PM}_{10}$ concentration, there is a $0.74 \%$ increase in joint estimated risk on daily mortality. Even though the amount of increased risk linked to a slight increase in daily $\mathrm{PM}_{10}$ concentration may appear small, the number of deaths estimated to be associated with such increased risk can be significantly large on a yearly basis depending upon population size. One study investigated the health effects of air pollution in six different cities in the US [77]. The results showed that mortality rates were strongly associated with $\mathrm{PM}_{2.5}$ and $\mathrm{PM}_{10}$ levels rather than the particulate levels of particles with sizes between $\mathrm{PM}_{2.5}$ and $\mathrm{PM}_{10}$.

Other studies which focused on the correlations between hospital admissions and air pollution were also conducted over the last few decades. One such study found that the previous day's 24 hour average $\mathrm{PM}_{10}$ concentration levels had a significant impact on the number of hospital emergency room visits for asthma in Seattle, WA [78]. Additionally, no evidence was found that related sulfur dioxide and ozone levels to the emergency room visits for asthma. Another study investigated the increase of hospitalization rate and/or emergency room visit frequency for respiratory conditions associated with exposure to $\mathrm{PM}_{2.5}$ in California Central Valley [78]. The study suggested that every $10 \%$ increase of $\mathrm{PM}_{2.5}$ concentration was associated with a $7.5 \%$ and a $4.1 \%$ increase in chronic and acute respiratory hospitalizations, respectively, as well as a $6.5 \%$ and a $5.2 \%$ increase in chronic and acute respiratory emergency room visits, respectively.

Diesel engine laboratory emission studies show an increase in nanoparticle emissions from low PM emission engines, usually equipped with DOCs and/or DPFs [40]. With the enforcement of new emission regulations, special concerns have been raised regarding the health effects of DPM pollution in the ultrafine and nanoparticle size 
ranges. A carbon black and diesel exhaust particle bioreactivity study was conducted which found the smaller particles to be more toxic and have larger surface areas [79]. Another study illustrated that fine and ultrafine particles were associated with increased mortality. However, the ultrafine particles showed more delayed effects than the fine particles with a lag time of about four days [80]. In another study, it was shown that ultrafine particles damage macrophage phagocytosis more severely than fine particles compared on a mass basis [81]. A further study demonstrated ultrafine elemental carbon (EC) particles trans-located to the liver after one day's inhalation exposure in animal studies [82]. In summary, ultrafine particles and nanoparticles may be of more concern regarding health effects because they may be more toxic on a per mass basis, have a better correlation with hospitalizations, and can penetrate into the body more easily.

\subsection{Near-Roadway and On-Roadway Studies}

As levels of air pollution in the atmosphere have increased, governmental agencies and institutions such as the EPA, CARB, OSHA, MSHA, and NIOSH have regulated ambient emission concentrations and personal exposure. Because it is argued that $35 \%$ of ambient $\mathrm{PM}_{2.5}$ is contributed by mobile sources [33], there has been increasing interest in the possibility of negative health effects due to elevated exposures near roadways [34,35]. In order to investigate this suspicion further, much research has been performed to quantify near roadway exposures by measuring ambient pollution concentrations near roadways and by understanding how the vehicle exhaust plume reacts once it is discharged from a vehicle exhaust system.

In a recent document regarding ambient pollution monitoring strategies [35], the EPA stated that it recognizes the importance of near roadway exposure and is attempting to add near roadway pollution monitoring capabilities to the already existing ambient air monitoring network. In an effort to begin quantifying this type of exposure and understanding the near road environment, a traffic-related exposure (T-REX) study [83] and a Detroit Exposure and Aerosol Research Study (DEARS) [84] were conducted near major roadways. Those studies compared the concentrations of air toxics and PM at central sites located near major roadways in New York City and Detroit to numerous personal and residential locations nearby in addition to measuring pollutant 
concentrations at 10,50,100, and $300 \mathrm{~m}$ from the roadway. It was found that indoor and outdoor PM concentrations were very similar with some component levels being lower indoors. It was also determined that pollutant levels decreased rapidly with distance from the roadway and that they decreased to background mass concentrations beyond $250 \mathrm{~m}$ from the roadway. In Los Angeles, particle size distributions, mass and elemental carbon concentrations were measured near roadways $[85,86]$. A four-fold decrease in particle number concentrations from a distance of $20 \mathrm{~m}$ from a roadway to $300 \mathrm{~m}$ from the roadway was observed. Several other studies have also found similar correlations between decreasing particle number concentrations and distance from roadways $[87,88]$.

It has also been shown by near roadway monitoring that pollutant concentrations vary with vehicle type and operating conditions [87,89-92]. Some studies observed higher concentrations of ultrafine particles when vehicle speeds and the proportion of heavy-duty vehicles on the roadway increase [87,89]. Another study [92] observed that the highest particulate concentrations and emission rates were linked to heavy engine acceleration, high engine speed, and high torque.

Numerous other studies have been more concerned with personal exposure at locations even closer to roadways, such as exposure inside a vehicle or beside a road. For example, a study conducted by the EPA [93] monitored in-vehicle pollutant concentrations for 10 police cars from the North Carolina State Highway Patrol. Pollutant concentrations were found to be orders of magnitude greater than at an ambient location outside the patrol station. The CARB released a similar study in 1998 [94] where invehicle air toxic concentrations were measured for vehicles in Los Angeles and Sacramento, CA. This study also found pollutant concentrations higher inside vehicles than at ambient locations.

Other studies have been conducted which focus more on public transit such as transit and school buses. Several studies monitored pollutant concentrations in diesel and natural gas school buses in Los Angeles [95,96]. These studies also found pollutant levels to be higher inside the buses compared with a fixed urban background site in west Los Angeles. However, a high amount of exposure variability was observed likely due to bus type, traffic congestion, and encounters with other diesel vehicles. The same researchers also attempted to determine whether or not school bus-related microenvironments had 
much importance regarding children's exposure, such as inside school buses during commute, at bus stops along the route, at bus loading and unloading zones, and at nearby background locations [97]. Depending on the pollutant considered, mean exposures inside school buses were found to be 50 to 200 times greater than at school unloading and loading zones, and 20 to 40 times greater than at bus stops along the bus route. Additionally, a number of other studies which focus on the exposure to pollutants inside passenger cars during commute found that pollutant levels were higher inside the vehicles while they were on busy roads [93,98-102].

In addition to pollution monitoring studies regarding in-vehicle exposure, there have been a number of studies that focus on exposure to pedestrians and bicyclists. Although it may seem more likely that pedestrians and bicyclists would have high exposures because they are very close to the roadway, existing literature show that exposure to pollutants in vehicle exhaust is greater for people inside vehicles compared with exposure for people outside of vehicles and near roadways. For example, one study found concentrations of PM that were 16\% higher inside a car than for a person walking on the same route [103]. However, it was noted that the person walking may have a larger overall exposure due to the longer traveling time. Similarly, another study found that car drivers were exposed to higher concentrations of traffic-related pollutants than cyclists [102]. However, when cyclists' breathing rates were considered, intake of pollutants approached that of the car drivers.

Many other similar studies have been conducted in Europe and Asia. For example, in Northampton, UK, personal measurements of exposure to $\mathrm{PM}_{10}, \mathrm{PM}_{2.5}$, and $\mathrm{PM}_{1}$ were made during walking and inside a car along two suburban routes [103]. Although only background concentrations for $\mathrm{PM}_{10}$ were available, it was found that incar measurements were highest $\left(43.16,15.54\right.$, and $7.03 \mu \mathrm{g} / \mathrm{m}^{3}$ for $\mathrm{PM}_{10}, \mathrm{PM}_{2.5}$, and $\mathrm{PM}_{1}$, respectively) followed by walking $\left(38.18,15.06\right.$, and $7.14 \mu \mathrm{g} / \mathrm{m}^{3}$ for $\mathrm{PM}_{10}, \mathrm{PM}_{2.5}$, and $\mathrm{PM}_{1}$, respectively) with background concentrations being significantly lower (26.55 $\mu \mathrm{g} / \mathrm{m}^{3}$ for $\mathrm{PM}_{10}$ ). Even though the literature has shown that in-vehicle exposure to pollutants in vehicle exhaust is typically higher than for pedestrians and bicyclists near roadways, the exposure to people in close proximity to roadways is still significantly higher than background levels. 
In addition to these types of exposure studies, many have been conducted which focus on near roadway exposure to motor vehicle exhaust due to occupational proximity. For example, several studies sought to quantify the exposure experienced by toll booth workers $[104,105]$. A number of studies conducted in Taiwan measured toll collectors' exposures to PM and PAHs using air and biological monitoring. One study [104] focused on sampling during different work shifts in order to correlate traffic flow rate with PAH levels. Highest PAH levels were found during the work shift associated with the morning rush. Another study [105] measured elemental and organic carbon concentrations for different lanes of traffic moving through a toll plaza in Taiwan. As expected, it was found that the bus and truck lanes had higher concentrations of PM than did the car lanes. Recognizing the potential pollutant exposure and corresponding health risks presented by the previous studies, another study [106] set out to evaluate the effectiveness of current Baltimore tollbooth ventilation systems in protecting tollbooth workers from elevated exposure to pollutants in vehicle exhaust. The 2 to 4 fold reduction in outdoor to indoor pollutant concentrations led researchers to believe that the ventilation systems in the Baltimore Harbor Tunnel tollbooths were adequate in reducing exposure.

As mentioned above, some researchers have approached the issue of exposure to vehicle exhaust by measuring pollutant concentrations near roadways, in vehicles, and beside roads; others have approached the issue by trying to determine what happens to exhaust once it is discharged from a vehicle through exhaust plume characterization and understanding. Some of the first research in this area was conducted by the Particle Technology Laboratory at the University of Minnesota with the measurement of particle distributions near the Harbor Freeway in Los Angeles, CA [107]. Using the Air Resources Board Mobile Air Pollution Laboratory, it was found that a significant amount of aerosol was contributed by the passing traffic on the freeway and a large nuclei mode referred to as a combustion mode was observed.

Other research in this area was launched in 1987 with the development of a remote sensing technology by the University of Denver [108]. This technology originally used an infrared sensor to emulate a typical non-dispersive infrared (NDIR) analyzer. The first sensor developed could detect only CO. Using this sensor, the researchers were able to determine what percentage of the ambient $\mathrm{CO}$ was due to the passing vehicles and 
consequently, determine whether or not the vehicles were running stoichiometrically. The sensor was used to measure CO emissions from on-road vehicles in Denver, Colorado Springs, and Chicago over several years [108]. Later, the ability to detect $\mathrm{CO}_{2}, \mathrm{HC}$, NOx, SOx, and other compounds found in vehicle exhaust were added to the original sensor created by the University of Denver and emissions were measured for fleets of on-road vehicles in additional US cities over 8 years from 1990 to 1998 [109-111]. Funded by the CRC under the E-23 program, the sensor was also used to measure emissions from onroad vehicles in four US cities several times each over the course of 8 years from 1998 to 2006 [112]. Thus, over the course of 19 years the sensor designed by D. Stedman and G. Bishop at the University of Denver measured emissions from on-road vehicle fleets in over 21 countries and 25 US locations.

At roughly the same time as the initial studies conducted by the University of Denver, the University of Minnesota conducted several studies measuring the on-road particle number, mass, and volume concentration, particle size distribution, and other quantities in the plumes of highway tractors [113]. The findings from those studies have become the basis for what is known about the evolution of exhaust plumes as they are discharged from tailpipes or stacks. The University of Minnesota continued this research as part of the E-43 program, funded by the CRC, by setting out to better understand the nature of emissions in the tailpipe to nose process occurring on and near roadways [40].

In that program, the University of Minnesota used a variety of measurement techniques to complete vehicle chase studies with a mobile emissions laboratory, wind tunnel studies, and chassis and engine dynamometer studies. Through those studies it was found that a stronger correlation existed between local traffic patterns and particle number concentration than with particle mass concentration [38]. Additionally, more nanoparticles were observed when traffic speeds were high. From on-road chase studies, it was found that all of the vehicles tested had bimodal particle distributions. Old and new technology vehicles had similar nuclei modes but the new technology vehicles had fewer accumulation mode particles. On-road dilution was on the order of 1000:1 in 2 seconds, and volatile compounds composed the bulk of the aerosols [38]. Wind tunnel studies [36], conducted in conjunction with West Virginia University, showed much lower dilution ratios, in addition to a unimodal particle distribution rather than a bimodal 
distribution. In a subcontract with Carnegie Melon University, on-road particle emission data were used with a dispersion model to simulate evolution of the plume particle distribution as it diluted and mixed with ambient air. It was computed that the time required for a plume to disperse $90 \%$ and $99 \%$ of the initial particle number took 20 to 30 minutes and the distance these particles can travel in this time ranged from $100 \mathrm{~m}$ to 10 $\mathrm{km}[40]$.

In addition to those studies, several studies were conducted which exposed rats in varying degrees of health to vehicle exhaust emissions during on-road chase experiments [114-116]. It was found that on-road exposure experiments such as these were indeed feasible, and it was noted that there did not appear to be a difference between responses from rats exposed to gas phase emissions and response from rates exposed to the gas phase/particle mixture [116]. It was also determined that many of the symptoms observed in the rats agree with those found in human exposure studies [114]. Additional reports based on the many studies performed using the University of Minnesota's mobile emissions laboratory reported the findings from all on-road diesel and spark ignited engine experiments [117,118].

\section{Experimental Setup and Procedures}

The experimental test setup used to carry out this study consisted of several pollutant measurement analyzers, a diesel fueled truck, a gasoline fueled truck, a device to record engine control unit (ECU) data, two laptop computers to record ECU data and data from the measurement equipment, and a mannequin and stroller used as pedestrian surrogates to observe adult and child exposure scenarios. The pollutant analyzers were used to measure concentrations of $\mathrm{PM}, \mathrm{CO}_{2}, \mathrm{CO}$, and NOx continuously as one of the two test vehicles was driven past the sampling site under known operating conditions. The particulate analyzer and the air pump it requires were strapped to a wooden pallet for easy transportation to and from the sampling site. The gaseous analyzers were strapped to a rolling cart for easy transportation to and from the sampling site also. Several gas bottles used for calibration were fixed to dollies for ease of transport to and from the sampling site. These arrangements were set around a foldable table where one data 
logging computer and the power supply were located. The entire arrangement can be seen in Figure 3-1.

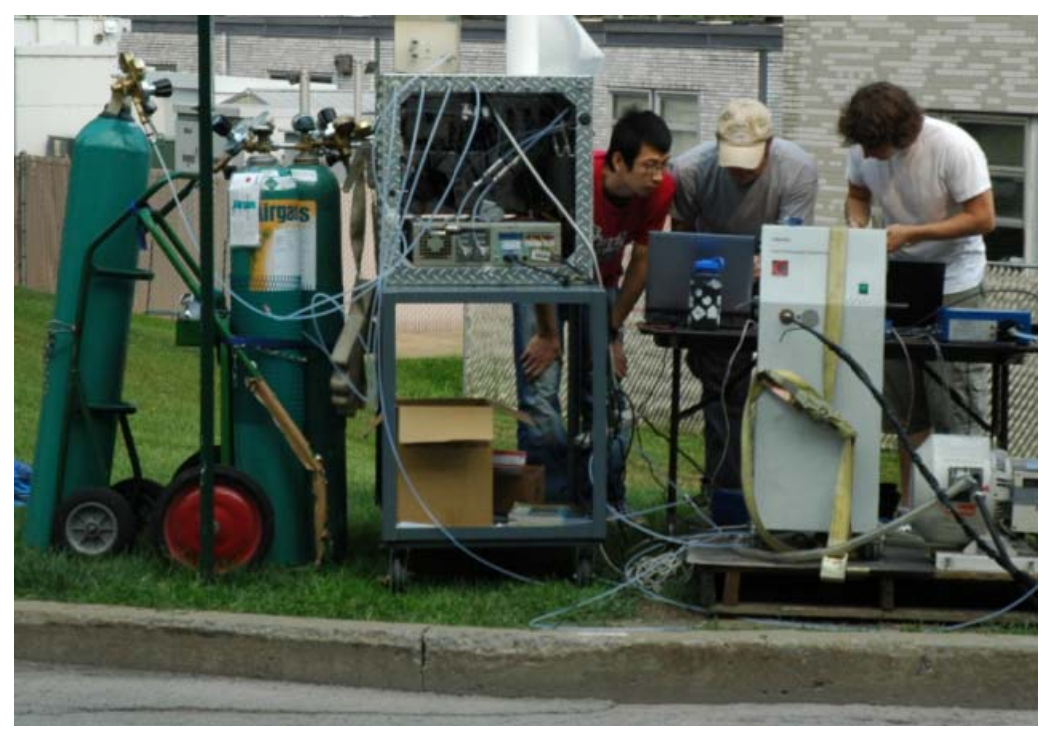

Figure 3-1: Experimental test setup.

\subsection{Emissions Measurement}

In order to mimic pedestrian exposure to near tailpipe exhaust emissions due to a vehicle drive-by accurately, gaseous and particulate emissions data were collected using non-heated sampling lines attached to the mouth of a lifelike mannequin. $\mathrm{PM}, \mathrm{CO}, \mathrm{CO}_{2}$, and NOx were measured continuously using a Cambustion DMS500 Fast Particle Spectrometer, a Horiba AIA-220 CO/CO 2 analyzer, and an EcoPhysics CLD-822 NOx analyzer, respectively.

\subsubsection{Cambustion DMS500 Fast Particle Spectrometer}

The Cambustion DMS500 Fast Particle Spectrometer, shown in Figure 3-2, is a mobility-based particle sizing instrument used to count particles between 5 and 1,000 nanometers in electrical mobility diameter. The DMS500 operates by charging each particle precisely using a corona discharge as it flows into a strong electrical field contained inside a classifier column. This electrical field deflects the charged particles towards the electrometer detectors depending upon each particle's aerodynamic drag/charge ratio (electrical mobility). When the particles contact the detectors at 
different points throughout the column, the increase in current due to each particle's charge is measured. The outputs from the 22 electrometers are then processed in real time to provide spectral equivalent diameter data and other desired particle parameters.

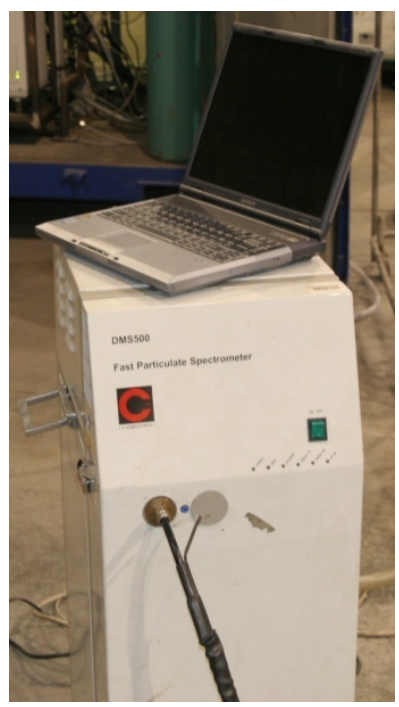

Figure 3-2: Cambustion DMS500 Fast Particle Spectrometer (cambustion.co.uk).

Although the particles in diesel exhaust do not have a uniform density and are not always spherical, both spherical shape and constant density are typically assumed when estimating mass using sample data from instruments designed to measure particle number-weighted size distribution via particle mobility [119]. For example, the TSI Engine Exhaust Particle Sizer ${ }^{\mathrm{TM}}$, a mobility-based instrument that operates on the same principle as the DMS500, requires the assumption of spherical particles of unit density to calculate mass [120]. Mobility-based instruments are based on Stokes' law which can be used to determine each particle's equivalent spherical diameter. The equivalent spherical diameter defines the equivalent diameter of a spherical particle of unit density with the same settling velocity as the collected particles [119].

Though this approach has been used by some [120], others have instead developed more empirical relationships between the electrical mobility diameter of the particles and the mass of the particles. A recent study [121], which compared the DMS500 and a Scanning Mobility Particle Sizer (SMPS), described the development of the following relationship between particle size and mass for the DMS500:

$$
\operatorname{Mass}(\mu \mathrm{g})=1.54 \times 10^{-16} \cdot D_{\text {eme }}^{3.19} \quad \text { Eq. } 1
$$


where $D_{\text {eme }}$ is the electrical mobility equivalent diameter in nanometers. Both of these approaches for calculating mass from DMS500 particle number data were applied to the data from this study and compared to each other.

\subsubsection{Horiba AIA-220 $\mathrm{CO} / \mathrm{CO}_{2}$ Analyzer}

The Horiba AIA-220 analyzer, installed in a custom measurement system, measured $\mathrm{CO}$ and $\mathrm{CO}_{2}$ continuously using nondispersive infrared (NDIR) technology. The custom measurement system consisted of an aluminum container about $2 \mathrm{ft}$ on all sides which housed the analyzer, a pump to control and provide adequate sample flow, a particulate filter to remove any particles that may damage the instrument, a cooler to remove all water vapor from the sample, and stainless steel transport tubing wrapped in heated insulating wire. Including the analyzer, the measurement system used a sample flow rate of about 7 liters per minute.

Before testing began, the Horiba AIA-220 analyzer was calibrated using NIST traceable $\mathrm{CO}_{2}$ and $\mathrm{CO}$ gases and linear regression using 11 points over a range from 0 to $2.003 \%$ for $\mathrm{CO}_{2}$ and 0 to $99.8 \mathrm{ppm}$ for $\mathrm{CO}$. To do this, both the measured concentrations and the corresponding analog voltage outputs were recorded. Second order quadratic polynomial regression equations were developed from these calibrations and used to predict concentrations from recorded voltages. The calibration data and the plotted quadratic regression equations can be seen in Appendix A. The concentration range used for calibration of $\mathrm{CO}$ was chosen based on expected concentrations in the vehicle exhaust. The concentration range used for calibration of $\mathrm{CO}_{2}$ was chosen because it was the lowest concentration available at the laboratory. During testing, the analyzer was zeroed and spanned every hour. Zeroing the analyzer was accomplished by flooding the analyzer probe with pure nitrogen gas. Similarly, the analyzer was spanned by flooding the analyzer probe with NIST traceable gases.

\subsubsection{EcoPhysics CLD-822 NOx Analyzer}

The EcoPhysics CLD-822 analyzer measured NOx continuously using the principle of chemiluminescence. This analyzer was used separately, without a custom measurement system using only the internal pump of the analyzer and had a flow rate of approximately two liters per minute. Before testing began, the EcoPhysics CLD-822 
analyzer was calibrated using NIST traceable NOx gas and linear regression using 11 points over a range from 0 to $101 \mathrm{ppm}$. To do this, both the measured concentrations and their corresponding analog voltage outputs were recorded. A linear regression equation was developed from the calibrations and used to predict concentrations from recorded voltages. The calibration data and the plotted linear regression equations can be seen in Appendix A. The concentration range used for calibration was chosen because it was the lowest concentration available at the laboratory. During testing, the analyzer was zeroed and spanned every hour. Zeroing the analyzer was accomplished by flooding the analyzer probe with pure nitrogen gas. Similarly, the analyzer was spanned by flooding the analyzer probe with NIST traceable gas.

\subsubsection{Analyzer Response}

Although the Horiba and EcoPhysics analyzers are considered to be continuously integrated and nearly instantaneous by manufacturer specifications, in practice there is a substantial lag time. In a typical emissions measurement laboratory setup, these analyzers have been found to have response delays up to 15 seconds which are well known and documented [122]. However, the bulk of these delays are not caused by the analyzers alone, rather, they are also due to the flow of exhaust through a vehicle's exhaust system and travel time through the emission sampling system. According to manufacturer user manuals, the Horiba and EcoPhysics analyzers have delays of 0.5 to $10 \mathrm{~s}$ and less than 1 $\mathrm{s}$, respectively, from the time a sample enters the analyzer until it is detected by the sensor. The sample tubing used in this study was approximately $5 \mathrm{~m}$ long with an inner diameter of $3.2 \mathrm{~mm}$. The transport delays for the analyzer sampling systems were calculated using this information and Equation 2:

$$
T_{D}=\frac{V}{F}
$$

where $T_{D}$ is the transport delay, $V$ is the volume of the sample line and sampling system, and $\mathrm{F}$ is the sampling system flow rate. The transport delays were calculated to be approximately 3 and 11 seconds for the Horiba and EcoPhysics sampling systems, respectively. The time delays between sample collection at the probe inlet and sensor 
response are given by the analyzer specifications stated previously and are in addition to the transport delays presently discussed.

\subsection{Test Vehicles}

The diesel test vehicle was a 2006 Dodge Ram 2500 with a 5.9 L, 325-hp, 6 cylinder diesel engine that discharges its combustion products through an exhaust system outfitted with an oxidation catalytic converter. The gasoline test vehicle was a 2001 Dodge Ram 1500 with a 5.2 L, 230-hp, 8 cylinder gasoline engine that discharges its combustion products through an exhaust system outfitted with a three-way catalytic converter. The tailpipes of both vehicles discharge towards the passenger side of the vehicle behind the rear wheel. More test vehicles were not considered due to a lack of resources. The test vehicles can be seen in Figure 3-3.
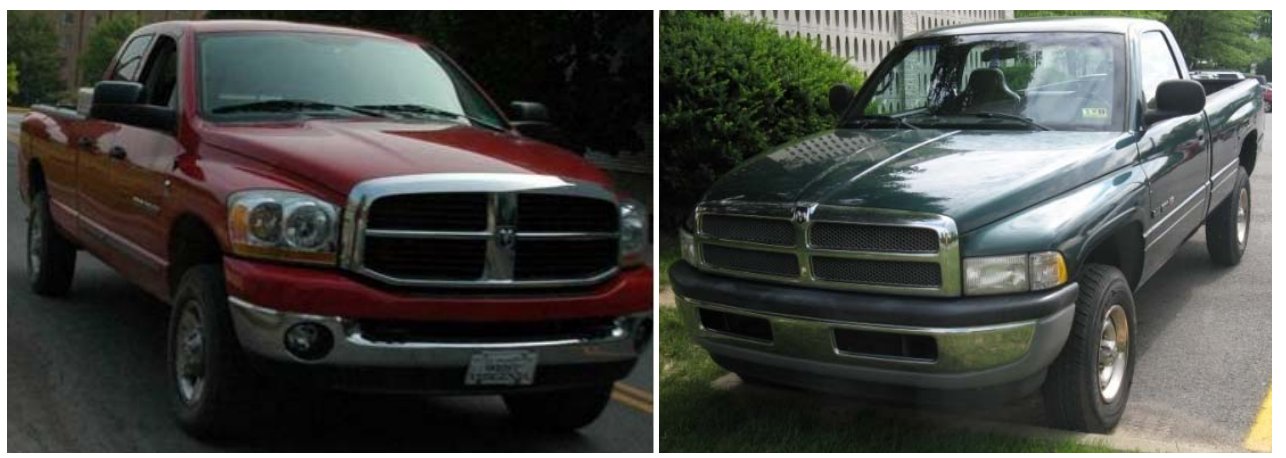

Figure 3-3: Test vehicles: Dodge Ram 2500 (left) and Dodge Ram 1500 (right).

\subsection{Engine Control Unit Data Logger}

An AutoTap OBDII Diagnostic Scanner, show in Figure 3-4, connected to an on board laptop computer running AutoTap software was used to monitor and record broadcast engine control unit (ECU) data. The broadcast ECU parameters recorded include the vehicle speed, engine speed, calculated percentage load, engine coolant temperature, and percent throttle position angle. Note that calculated percentage load is a broadcast parameter and was not calculated by the author. Also, note that throughout the results the engine power is referred to often. This parameter was calculated by the author using a torque versus engine speed curve for the engines of the test vehicles. The torque vs. engine speed curve and procedure used to compute the engine power are shown in Appendix B. The software allowed the vehicle operator to monitor in real-time the 
calculated percentage load which made it possible to maintain the desired engine loads of $100 \%, 50 \%$, and $0 \%$ when passing the sampling location. These loads were chosen to simulate real world driving conditions such as hard acceleration, medium acceleration, and vehicle cruising.

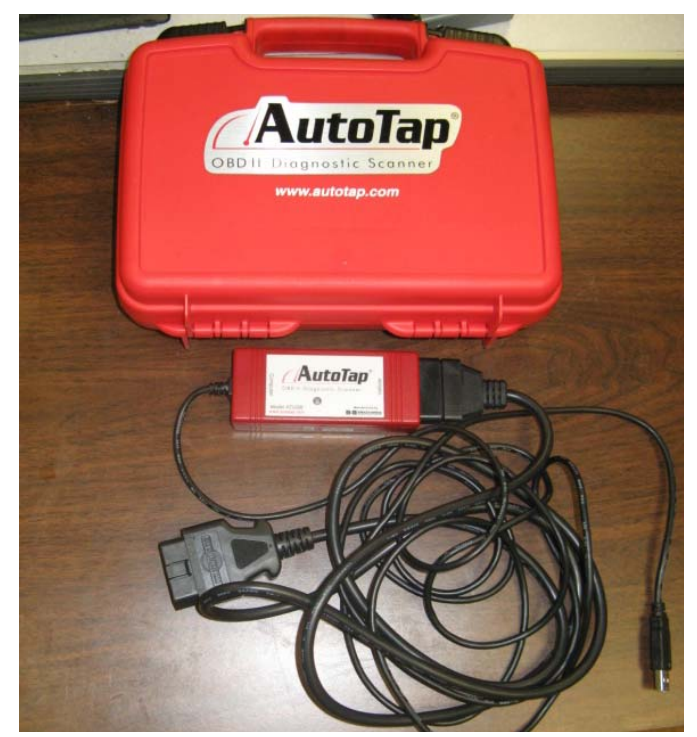

Figure 3-4: AutoTap OBDII Diagnostic Scanner.

\subsection{Pedestrian Surrogate and Sample Probe Support}

At the sampling location, a lifelike mannequin with a detachable Styrofoam ${ }^{\mathrm{TM}}$ head was used as a surrogate for human pedestrians. Note that the neck was extraordinarily long due to the detachable head. However, this abnormality was not expected to have a great effect on the results of the study. The mannequin can be seen in Figure 3-5. Including the head, the mannequin was about $1.8 \mathrm{~m}$ tall (i.e., approximately $1.65 \mathrm{~m}$ above the ground at the mouth). It had a rigid stance with no moving body parts except for detachable limbs and was held in a standing position by a metal stand which attached to the mannequin's calf. As shown in Figure 3-5, during testing the mannequin wore a maroon turtleneck long sleeve shirt and gray sweatpants. The mannequin was a custom model manufactured by Fusion Specialties and was purchased at a flea market.

Sample probes located in the mouth of the mannequin, Figure 3-6, extended though the back of the Styrofoam ${ }^{\mathrm{TM}}$ head and attached to Teflon ${ }^{\circledR}$ transport tubing $5 \mathrm{~m}$ in length connected to the analyzers. To mimic a child in a stroller, the mannequin's head, sample probes and all, were placed inside a stroller and positioned near the roadway. The full mannequin and the stroller setup can be seen in Figure 3-5. During this setup, the 
sample probes were approximately 0.85 meters from the ground. To support the Teflon tubing at the mannequin's head, a support stand constructed from 1" square cross sectional strut channel. The support stand was not only used to support the transport tubing; it was also used to compare the effects of the mannequin's body on measurements.
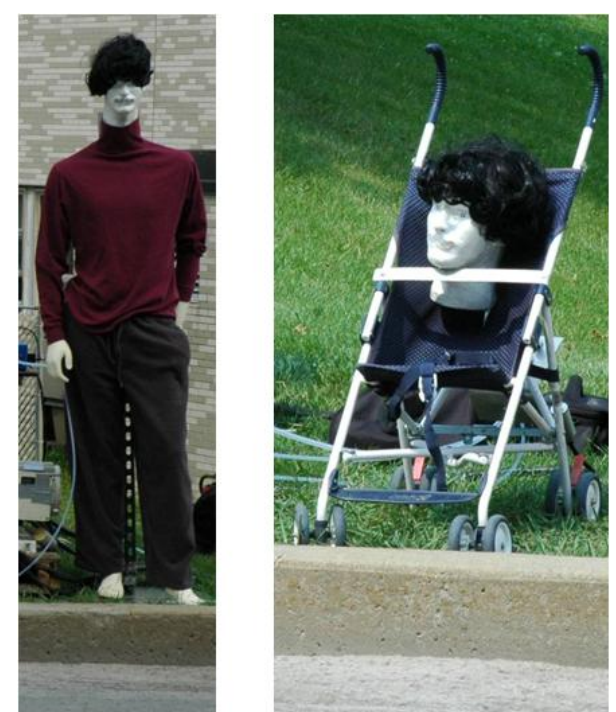

Figure 3-5: Pedestrian surrogate mannequin (left) mannequin's head simulation in stroller (right).
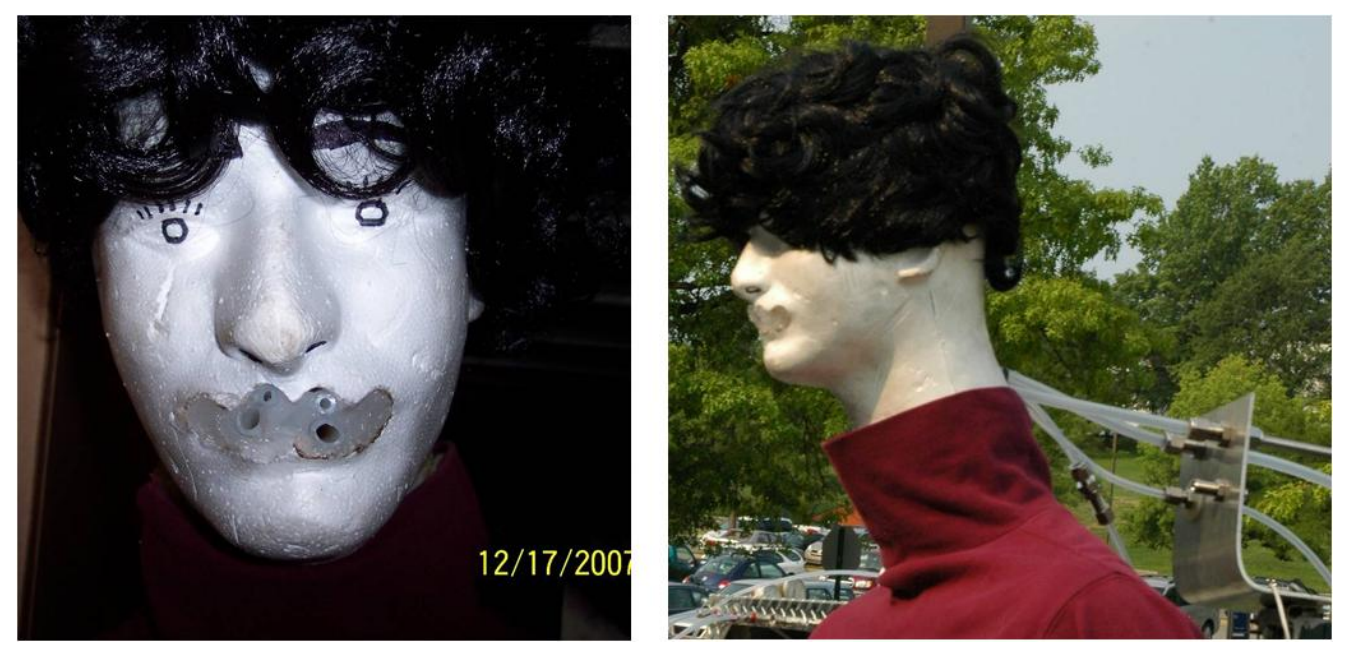

Figure 3-6: Mannequin's head with sample probes in mouth and attachment to sample tubing.

\subsection{Study Site}

The study was conducted during August 2007 in Morgantown, West Virginia on a two-lane road bisecting the WVU Evansdale campus. The study location was chosen for its minimal traffic congestion and the presence of an adequate power supply. 
Additionally, the land on one side of the road was relatively flat for about $5 \mathrm{~m}$, providing a simple topography that should be comparable to many urban or suburban sites. There was a single building nearby that could have caused a small urban canyon effect. However, preliminary measurements revealed that background concentrations of pollutants of interest were very low. Thus, it is unlikely that the building would prevent dispersion of the vehicle exhaust or affect measurements.

\subsection{Scenarios Observed}

Although the term "pedestrian" typically refers to a person walking or traveling on foot, for the purposes of this study the term was broadened to include persons who are near roadways and either walking or standing. Also, the term does not distinguish between individuals who are nearby for occupational or for personal reasons. Analyzers were placed along the test road to measure exhaust constituents at the mouth of a surrogate pedestrian. During periods of sampling, a diesel fueled vehicle was driven past the mannequin and the analyzers under different operating conditions.

Though there have been several studies to analyze near-tailpipe vehicle exhaust or near-roadway exposure [123-126], few have instrumented the vehicle to record ECU data during the drive-by incidents, as was done in this study. The instrumented mannequin was placed beside the roadway to simulate an adult male standing on the sidewalk next to a roadway. In addition, the mannequin's head was removed and placed in a stroller to simulate a child in a stroller. For the third test scenario, the mannequin was removed and the sample probes were attached to the support stand described earlier to allow for comparison of samples taken at the same height with and without the presence of the simulated human. This setup was considered because the shape and location of a person could affect the sample due to factors such as eddies caused by air flow around a body.

The three operating conditions that were varied when the test vehicle passed the sampling location were (i) acceleration at nearly $100 \%$ (full) load, (ii) acceleration at about 50\% (part) load, and (iii) cruising at a constant velocity with high engine speed. These operating conditions were chosen because all three commonly occur near pedestrians and could be expected to produce very different levels of particulate matter expulsion. Several studies [90-92] have shown that the formation of particulate matter 
and other diesel exhaust constituents varies greatly with engine operation. The highest particulate concentrations and emission rates observed in the third study were linked to heavy engine acceleration, high engine speed, and high torque [92].

The acceleration tests were accomplished by accelerating the vehicle from a rolling start $(5 \mathrm{mph})$ past the sampling location and reaching about $25 \mathrm{mph}$ while monitoring the engine load to ensure that the proper load was maintained while the vehicle passed the sampling location. The cruising tests were accomplished by accelerating the vehicle to approximately $25 \mathrm{mph}$ and maintaining a constant speed for at least 30 meters before passing the sampling location. During these drive-by tests, the vehicle operator attempted, when near the sample locations, to keep the vehicle at a distance of about $0.5 \mathrm{~m}$ from the curb and thus about $0.75 \mathrm{~m}$ horizontally from the sample probes. Four to six drive-bys were conducted for each combination of scenarios.

The drive-by incidents were conducted according to the diagram shown in Figure 3-7. The adult and child scenarios were setup such that the adult's mouth was approximately $1.02 \mathrm{~m}$ above the tailpipe of the passing vehicle, and the child's mouth was about $0.254 \mathrm{~m}$ above the tailpipe. Additionally, the pedestrians were approximately $0.152 \mathrm{~m}$ above the roadway as if they were on a sidewalk, typical of urban or suburban settings. Although it was not necessarily kept constant during testing, the edge of the vehicle's tailpipe was usually about $0.762 \mathrm{~m}$ horizontally from the plane in which the edges of the sampling tubes were located.

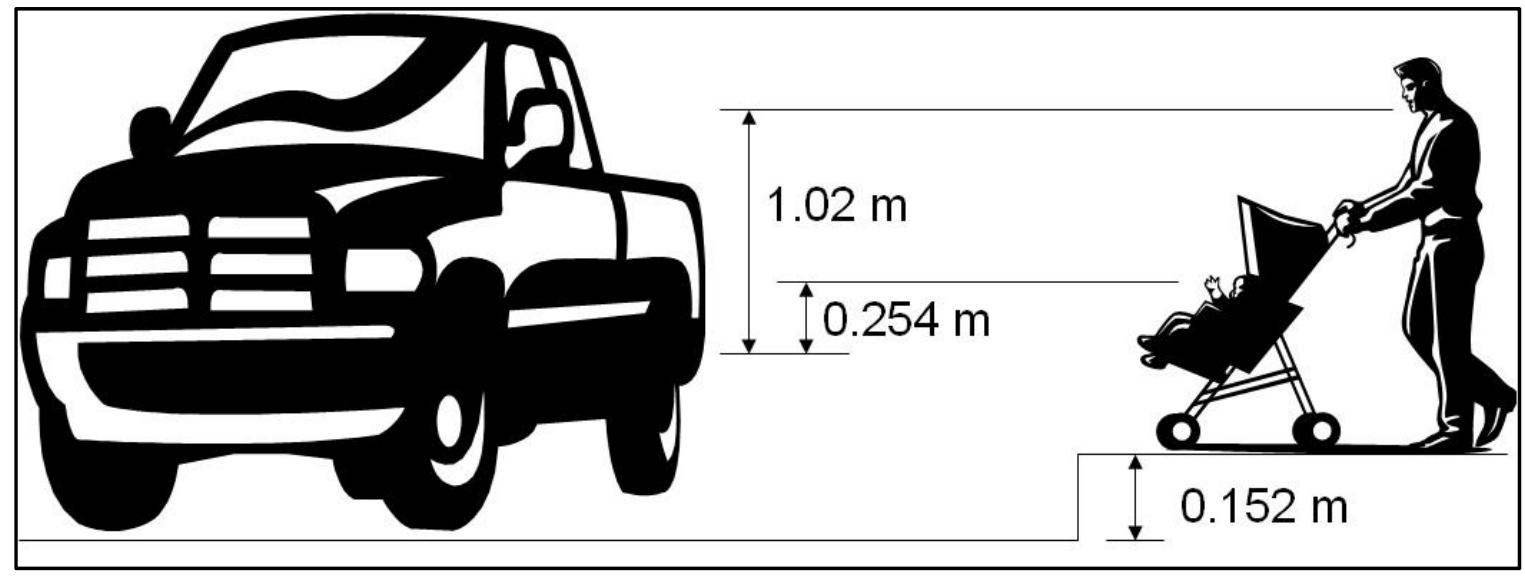

Figure 3-7: Diagram of drive-by experiment layout. 


\subsection{Pollution Monitoring}

Teflon sample lines from the analyzers were connected directly to the mannequin's mouth and run roughly $5 \mathrm{~m}$ to the sampling devices. Air was sampled continuously providing continuous emissions data as the test vehicle passed the mannequin during each test run. Data from all of the analyzers were recorded simultaneously by connecting the analog outputs of the Horiba and EcoPhysics analyzers to the analog inputs on the Cambustion DMS500 via modified coaxial cable. A program, supplied with the Cambustion DMS500, was used both to control the DMS500 instrument and to record the $\mathrm{PM}, \mathrm{CO}, \mathrm{CO}_{2}$, and $\mathrm{NOx}$ measurements. The resulting measurements were associated with specific test vehicle engine conditions by synchronizing the computer used to record analyzer measurements with the computer recording engine data. The time at which the vehicle passed the sampling point was recorded using a Microsoft Visual Basic ${ }^{\mathrm{TM}}$ program custom-written for this study by the author. Using these time-stamped data, the engine conditions during each test could be correlated with the emissions measured during each test. To ensure that emissions linked with the test vehicle were not affected by other vehicles, the drive-by runs were conducted when there were no other operating vehicles nearby.

Because the particle size range of the DMS500 typically accounts for 80 to 95 percent of the total particulate matter mass found in diesel exhaust [127], the resulting measurements can be assumed to approximate $\mathrm{PM}_{2.5}$ concentrations. Thus, PM measurements were taken to estimate $\mathrm{PM}_{2.5}$ exposure, $\mathrm{CO}$ was measured as an attempt to correlate $\mathrm{CO}$ concentrations with PM concentrations (see [128]), and $\mathrm{CO}_{2}$ and $\mathrm{NOx}$ were measured to help quantify the dilution ratio of the exhaust exiting the test vehicle's tailpipe.

\subsection{Data Reduction}

In order to be sure the data acquired during testing was adequate, the data were reviewed periodically throughout testing. After testing was complete and all the data had been acquired from testing, a program custom-written in Microsoft Visual Basic for Applications (VBA) ${ }^{\mathrm{TM}}$ was used to extract the desired data from text files and import it into a Microsoft Excel ${ }^{\mathrm{TM}}$ file. During extraction, the gaseous sample concentrations were 
calculated from the observed voltages using the corresponding calibration equations. In addition, the concentration of particulate matter in micrograms per cubic meter of air $(\mu \mathrm{g} / \mathrm{m} 3)$ was computed using the previously mentioned analyses correlating particle diameter and mass.

A vehicle's exhaust system and the emission sampling system both have time delays (up to 12 seconds combined) [122,129,130], although raw exhaust measurements, such as obtained in this study, typically have shorter time delays than standard dilution tunnel measurements [130]. Consequently, all figures containing this gaseous data had to be corrected based on the analyzer specifications as well as the measured sampling delays. By combining the transport delays and manufacturer specified delays mentioned previously, the delay for the $\mathrm{CO}$ and $\mathrm{CO}_{2}$ data was estimated to be approximately 3.5 seconds, and the delay for the NOx data was estimated to be about 12 seconds. These delays were required in order to correlate the emissions with specific engine operating conditions. However, the instantaneous pollutant concentration data obtained from analyzers are diffused in time [130] because they do not represent the instantaneous emissions that may arise due to a short lived engine operating condition. No measures were taken to rectify the data because the nature of the diffusion was unknown.

\subsection{Exposure Estimation}

Human exposure to diesel exhaust is typically considered as an average particulate matter concentration over a certain amount of time. For example, the MSHA exposure limit is a concentration averaged over an 8 hour period [32]. During drive-by incidents, the measured concentration of particulate matter is initially equivalent to the background concentration but quickly increases to a maximum as the exhaust plume reaches the sample lines. It then decreases back to the background concentration levels as the exhaust is diluted by mixing with ambient air.

In order to estimate the exposure pedestrians may experience, the instantaneous sample concentrations of particulate matter obtained at $5 \mathrm{~Hz}$ from the DMS500 were mathematically averaged over the duration of each drive by incident. For this study, the duration of an incident was defined as the time interval beginning when the exhaust plume from the tailpipe produced a noticeable increase in particulate matter concentration 
at the computer and ending when the exhaust plume had diluted sufficiently that the measured concentration of particulate matter was near background levels again. The noticeable increase in concentration or beginning of an event was determined by first computing the standard deviation of the background concentration for two to four seconds starting at the events time stamp. A three point ( 0.3 second $)$ running average of the concentration was then computed. If this value was greater than the average background concentration plus 10 times the standard deviation of the background concentration, the time associated with the second point in the three point average was considered to be the time the event began. The end of the event was similarly determined to be when the value of the running average was less than the average background concentration plus 10 times the standard deviation of the background concentration.

To compare the estimated mass inhaled during the drive-by incidents with the estimated mass inhaled at ambient conditions, maximum and minimum ambient conditions were specified. The ambient concentrations considered were equivalent to (i) the National Ambient Air Quality Standard and (ii) the ambient concentration in Darrington, Washington, a city which the EPA considers to have good air quality. These ambient concentrations of 35 and $5 \mu \mathrm{g} / \mathrm{m}^{3}$, respectively, represent reasonable maximum and minimum expected ambient concentrations of particulate matter.

Since the number of drive-bys in the study was fewer than the number pedestrians may experience on city sidewalks, the estimated exposures for typical pedestrians were determined by multiplying the average test values by a reasonable estimate of typical frequency of drive-bys. For the latter, it should be noted that the theoretical maximum traffic volume a single lane road can support is given by the ratio of vehicle speed and vehicle spacing [131]. Assuming a speed limit of $25 \mathrm{mph}$, such as that of the test road, and a vehicle spacing of 12.2 meters (approximately two car lengths), the maximum traffic volume that can be obtained is 3,300 vehicles per hour. Of course, not all vehicles are diesel-powered and gasoline engines also emit particles at measurable mass and number levels [132,133]; particle mass and number emissions from gasoline vehicles are orders of magnitude smaller than from diesel vehicles. Using the assumptions made in the EPA's MOBILE6 emissions model, it was determined that $1.02 \%$ of all light duty vehicles and class $2 \mathrm{~B}$ and class 3 heavy duty vehicles produced in the US in 2008 were 
diesel fueled [134]. This percentage implies that on average 33 diesel fueled vehicles pass a single point on a road, such as described above, every hour. Therefore, over an 8 hour period, a typical incident count could be as high as 264. Note that this 8 hour time period comes from an occupational exposure standard. Also, note that the diesel test vehicle used in this study is representative of class $2 \mathrm{~B}$ heavy duty diesel vehicles.

Another type of short-term exposure considered to be applicable to drive-by incidents, such as explored in this study, is exposure per inhalation. This type of shortterm exposure deals with single inhalations of very high concentrations. To determine this exposure as a worst case scenario, an incident from each scenario with the highest instantaneous concentrations was aligned with the inspiration of a breath. Inspiration lengths of 2.5 and 1.5 seconds were used to imitate walking and standing breathing rates, respectively. This maximum amount of particulate matter inhaled in a single breath during a drive-by incident was then compared to the amount of particulate matter inhaled in a single breath at the same ambient conditions stated previously.

\section{Results and Discussion}

\subsection{Drive-By Test Results}

The raw data from typical drive-by tests with the diesel pickup truck can be seen in Figure 4-1, Figure 4-2, and Figure 4-3. Figure 4-1 represents the case where the test vehicle accelerated past the mannequin at nearly full load. Figure 4-2 corresponds to the case where the test vehicle accelerated past the mannequin at part load. Figure 4-3 shows the case in which the test vehicle was driven past the mannequin at a constant velocity with high engine speed and low load. In these figures, the driving conditions of the test vehicle, namely engine speed, calculated percentage load, and power, as well as the concentrations of the desired exhaust constituents at the mouth of the mannequin were plotted versus time.

Although Figure 4-1, Figure 4-2, and Figure 4-3 show only one typical plot obtained from each driving condition considered with the mannequin, it can be seen that the curves representing instantaneous particulate matter concentration are quite different in each plot. This is because peak concentrations and durations of exposure incidents 
varied widely from test to test and because truck operation differed from case to case. Wind speed and direction greatly affected the peak concentrations measured and the durations of the exposure incidents by affecting the exhaust travel time from the tailpipe to the sample lines and varying the exhaust dilution and amount of residual entrainment in eddies. The vehicle operator also affected the repeatability of tests due to varied pedal commands and vehicle positioning with respect to the sampling point.

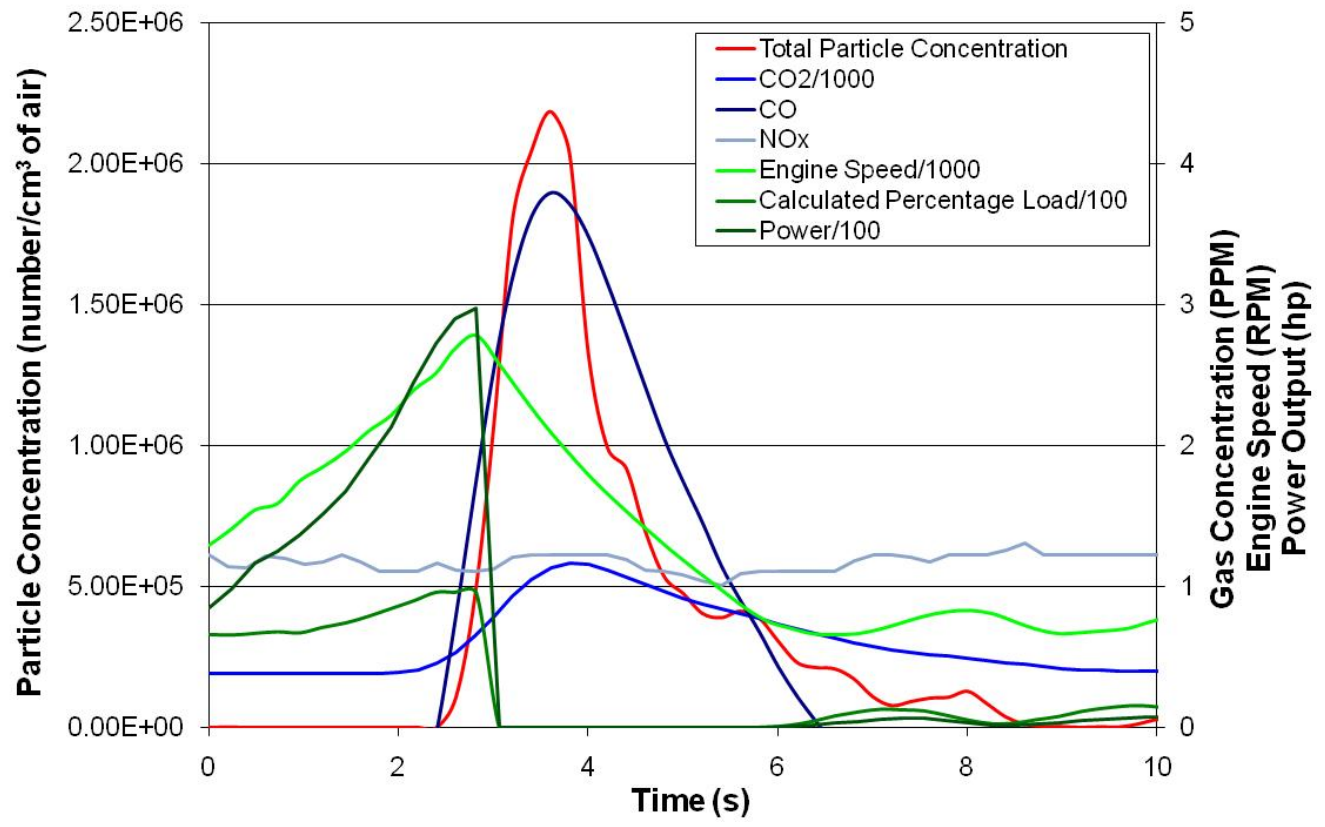

Figure 4-1: Raw data from hard acceleration drive-by with mannequin.

In Figure 4-1, the engine speed, calculated percentage load, and the power are all near the maximum of their respective ranges. As such, notice that the total particle concentration peaks in the low 2 millions. Also, notice that the $\mathrm{CO}$ and $\mathrm{CO}_{2}$ concentrations peak and return to background concentrations while there appears to be no peak NOx concentration. 


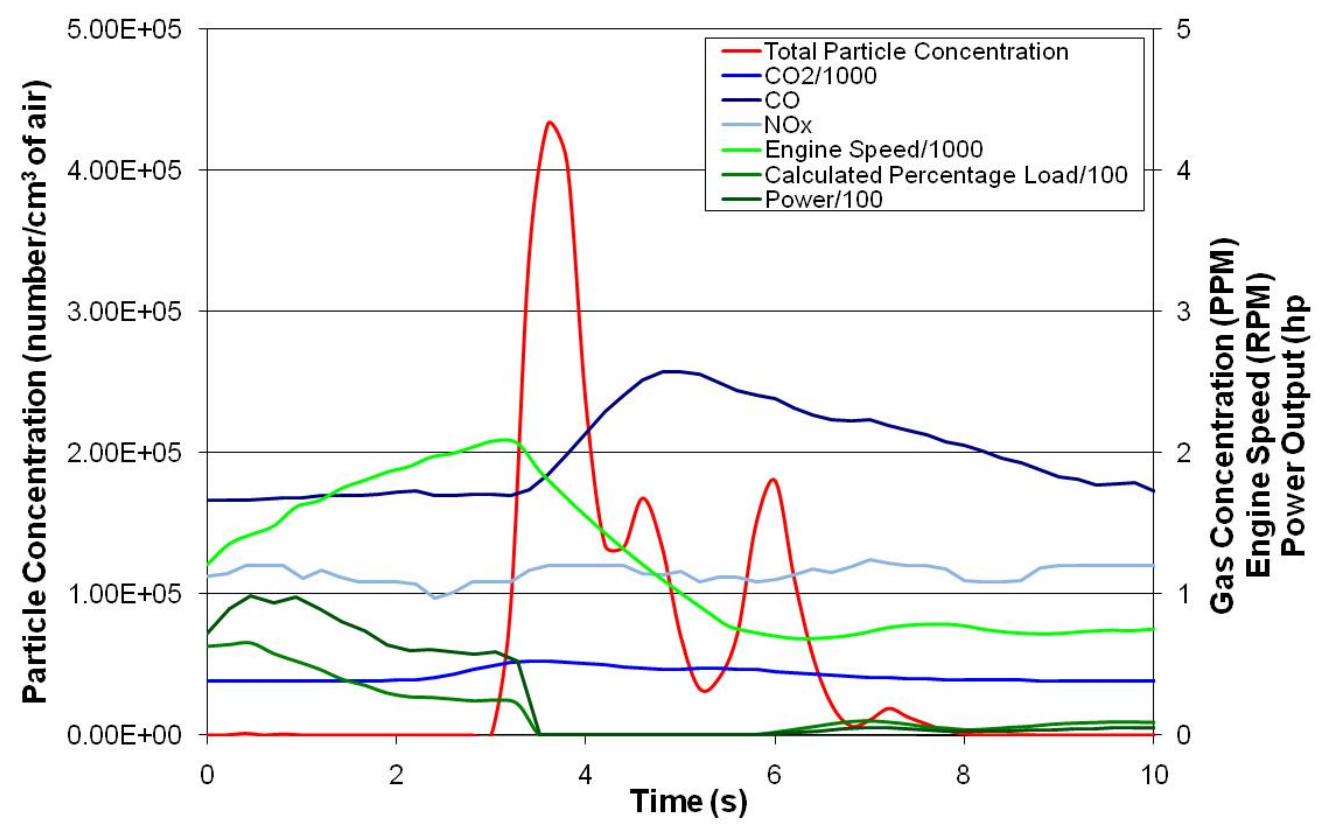

Figure 4-2: Raw data from medium acceleration drive-by with mannequin.

In Figure 4-2, it can be seen that the peak total particle concentration is much lower than the peak shown in Figure 4-1 as are the engine speed, calculated percentage load, and power. Additionally, the peak concentrations of $\mathrm{CO}$ and $\mathrm{CO}_{2}$ are also lower. However, the NOx concentration again appears to have no noticeable peak.

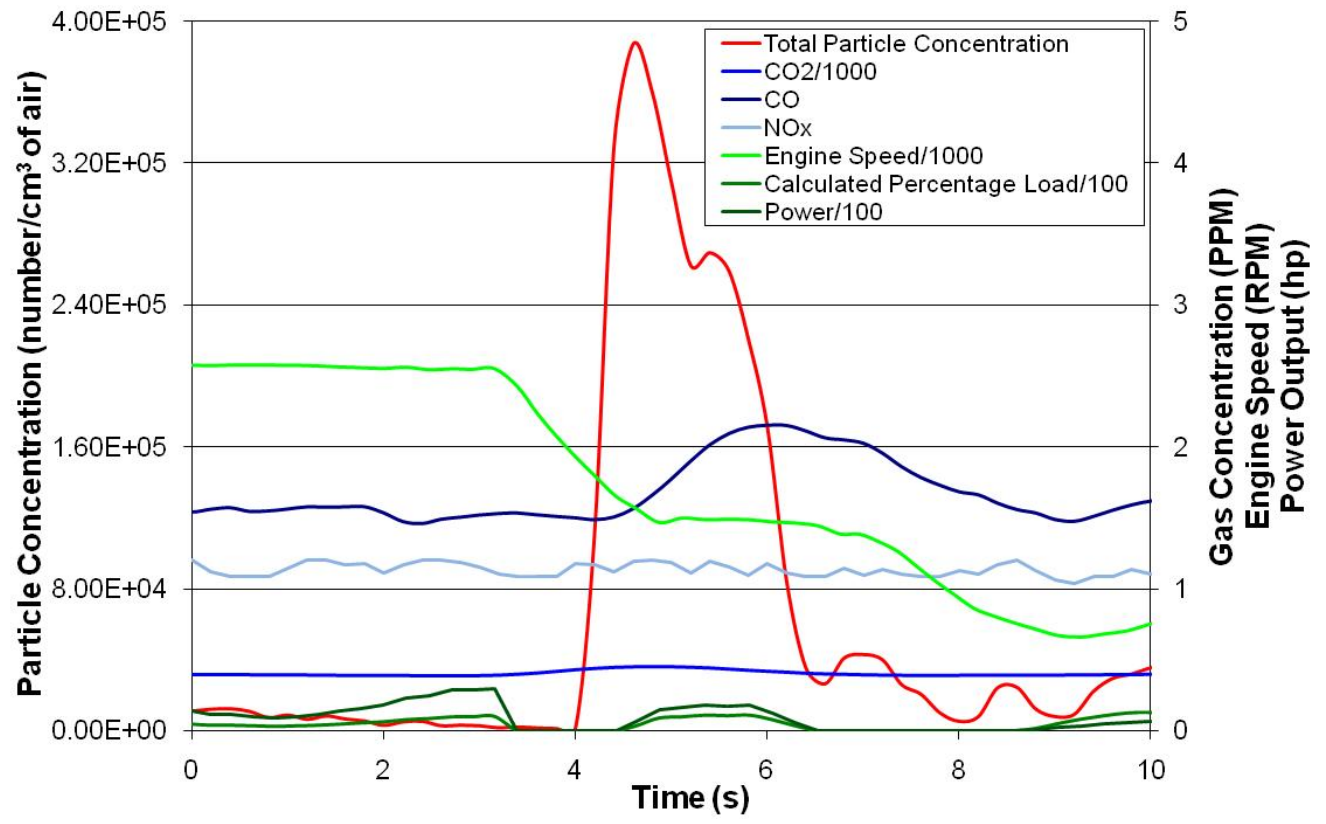

Figure 4-3: Raw data from cruising drive-by with mannequin. 
In Figure 4-3, it can be seen that the peak total particle concentration is even lower than in the previous to figures. However, in this case, only the calculated percentage load and the power are low. The engine speed is near the maximum of its range. Figure 4-1, Figure 4-2, and Figure 4-3 are significant because they show how that exhaust pollutant concentrations are dependent on vehicle engine operating conditions. In particular, high engine power and hard vehicle acceleration produce the highest levels of PM.

As discussed previously, two methods for computing the particulate mass were utilized in this study. One method was specifically developed for the DMS500 while the other was typical of mobility based particle sizing instruments assuming spherical particles of unit density. When comparing the results using these two methods, Figure $4-4$, it was found that the mobility based method was consistently 1.4 times higher than the DMS specific method. Despite this difference, the DMS specific method for calculating particulate mass was used for computational ease.

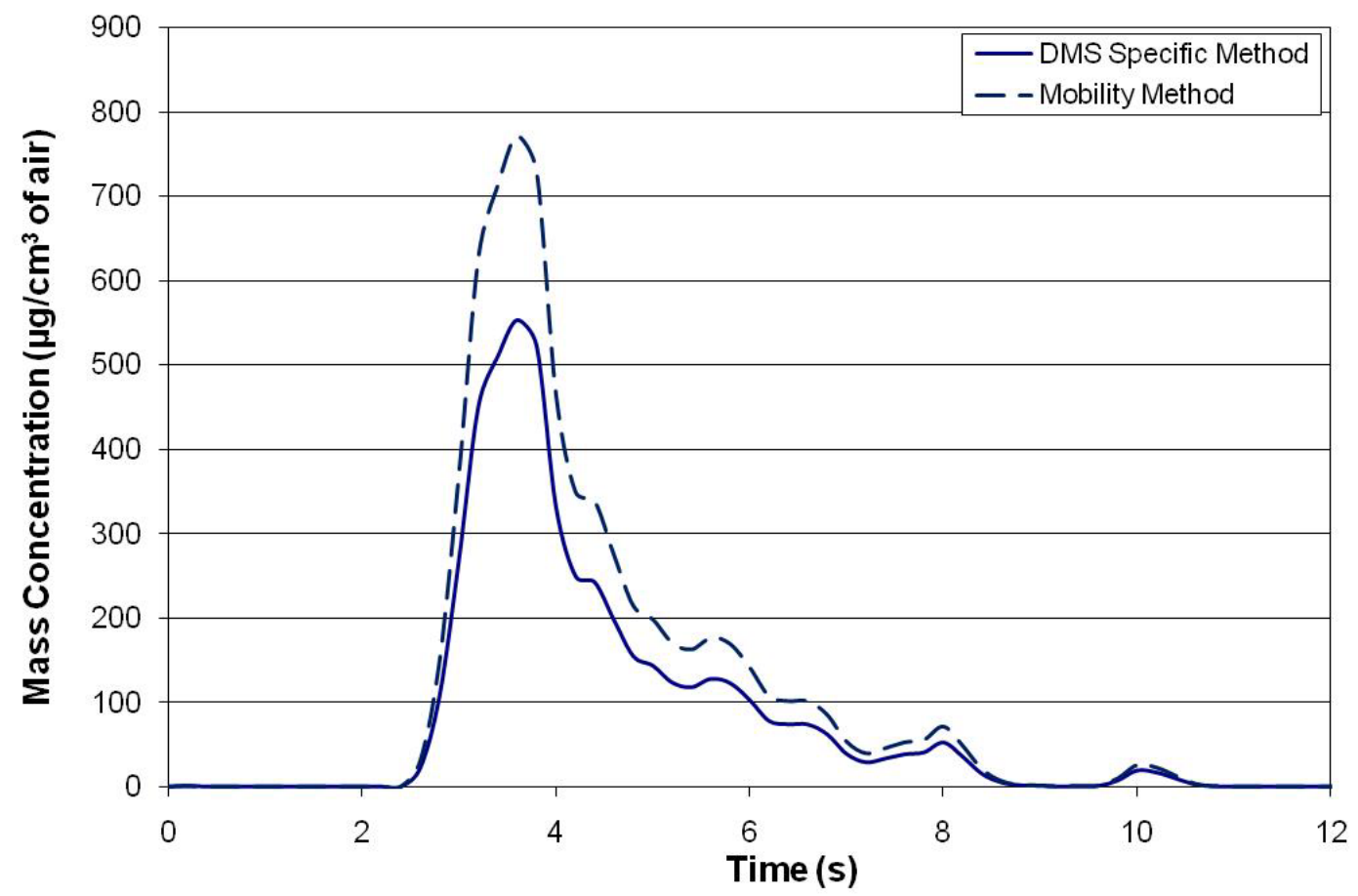

Figure 4-4: Comparison of mass concentration determination methods.

Using the method just discussed, the particle number and mass concentrations for all drive-by test runs performed were plotted and can be seen in Appendix C. In order to 
compare the diesel and gasoline vehicles, the number and mass concentrations from a full load acceleration test with each vehicle were plotted together in Figure 4-5. Note that the concentrations obtained with the diesel vehicle are some of the lowest encountered during full load acceleration tests with the diesel vehicle. Also, note that the concentrations shown for the gasoline vehicle are some of the highest observed during full load acceleration tests with the gasoline vehicle.

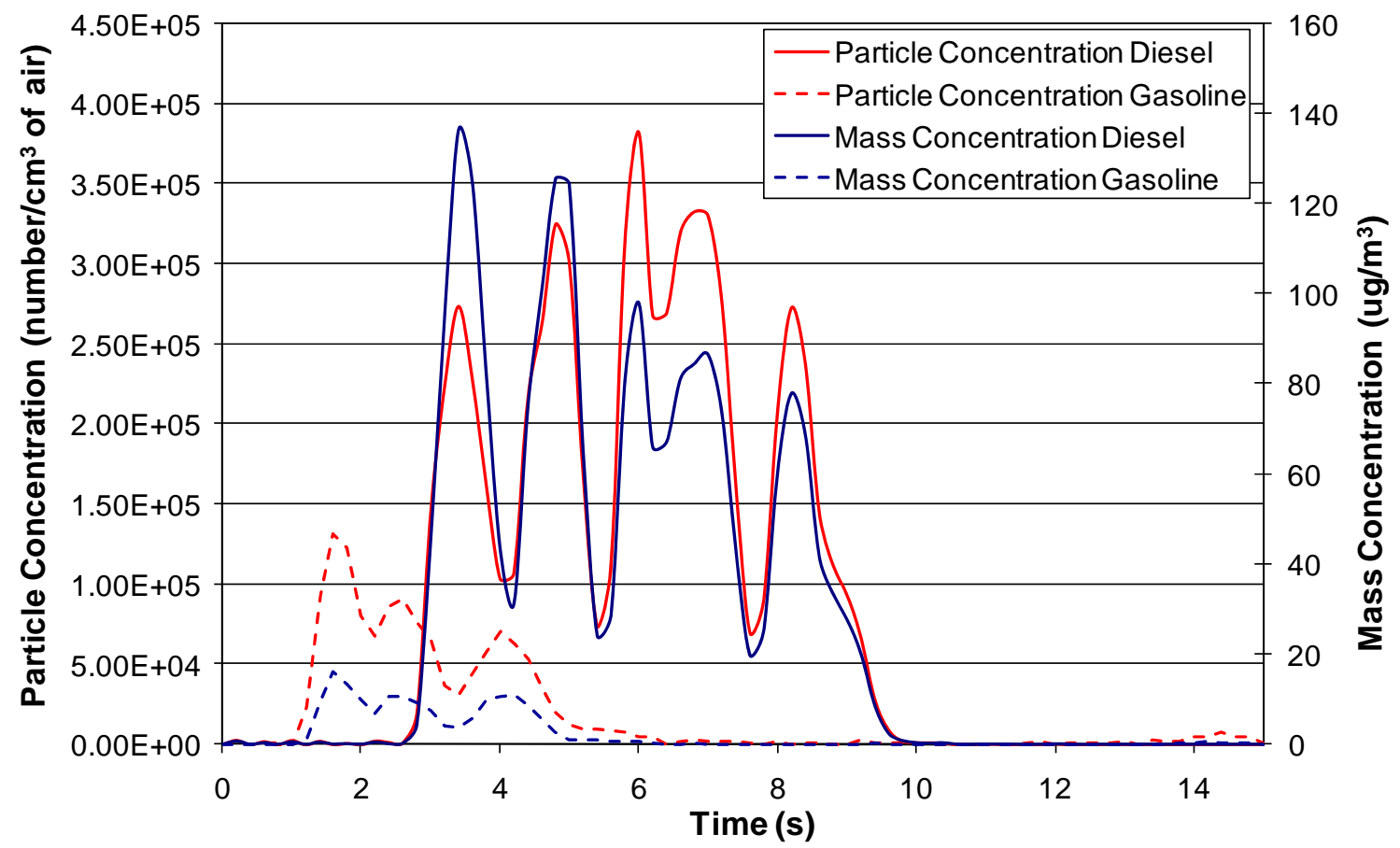

Figure 4-5: Particle number and mass concentrations for diesel and gasoline vehicle.

In Figure 4-5, it is apparent that the number concentration measured during the full load acceleration test with the diesel vehicle is on average four times higher than that obtained using the gasoline vehicle. It is also apparent that the mass concentration obtained during the diesel vehicle drive-by is on average an order of magnitude greater than that acquired with the gasoline vehicle. This shows that the lowest average concentrations attained with the diesel test vehicle are higher than the highest average concentration obtained with the gasoline vehicle. Unless a significant difference exists between the way in which exhaust exits the gasoline test vehicle and the diesel test vehicle, these results imply that the diesel test vehicle emits higher concentrations of both particle number and particle mass. 
In order to see which particle mode was most prominent (i.e. had the highest number of particles) and which particle mode was responsible for the most mass, the normalized particle number and mass concentration size distributions were plotted for Figure 4-5. The normalized number and mass weighted size distributions for the diesel vehicle can be seen in Figure 4-6 and Figure 4-7, respectively. The normalized number and mass weighted size distributions for the gasoline vehicle can be seen in Figure 4-8 and Figure 4-9, respectively.

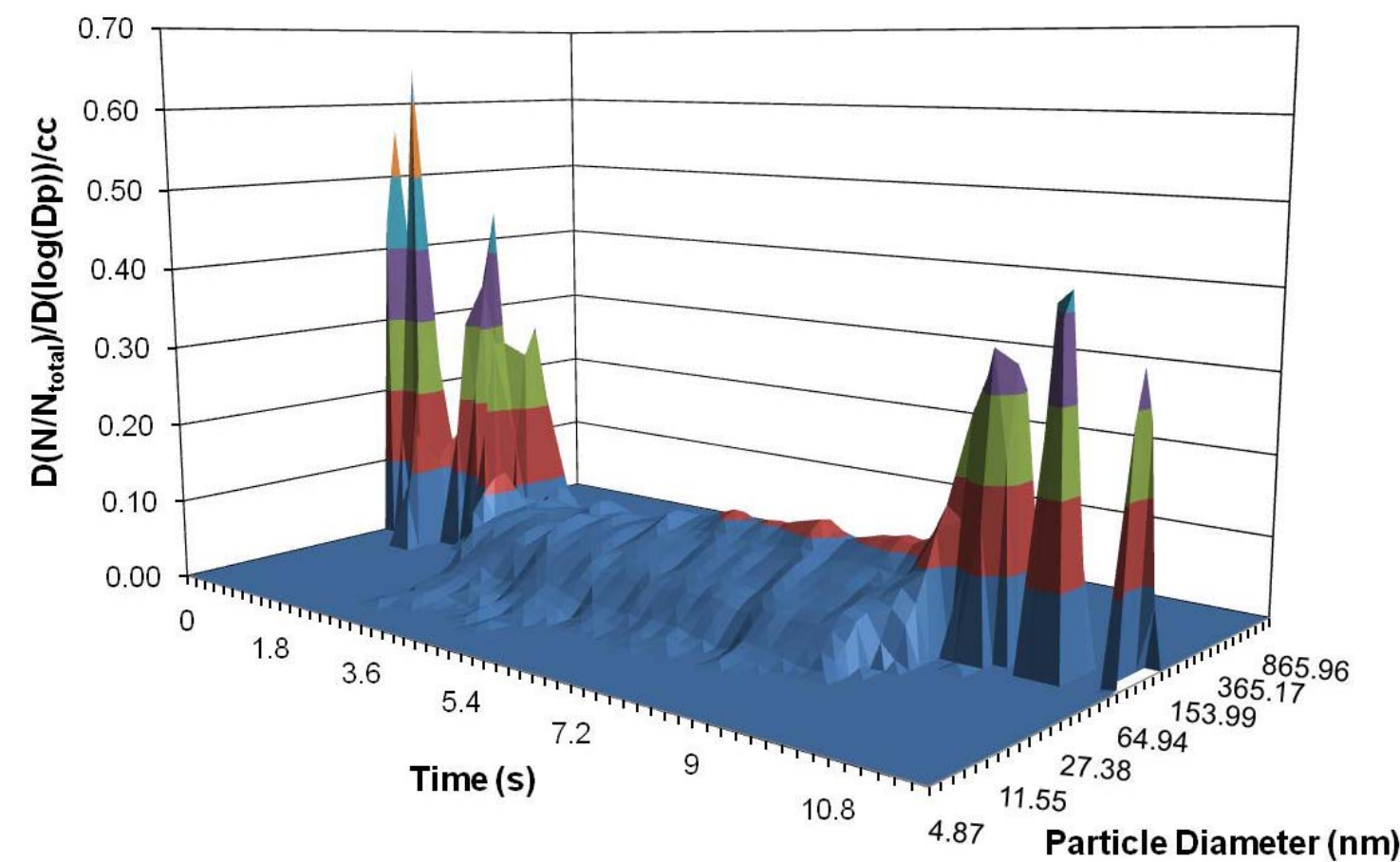

Figure 4-6: Normalized number weighted particle size distribution obtained with the diesel vehicle.

In Figure 4-6, it can be seen that before and after the diesel drive-by incident the normalized number weighted particle size distribution is dominated by accumulation mode particles about $100 \mathrm{~nm}$ in electrical mobility diameter. However, during the driveby incident (about 2 to 9 seconds) the normalized number weighted size distribution is composed of a combination of accumulation mode and nuclei mode particles. The absence of the typical bimodal distribution implies that the exhaust may have not been highly diluted. 


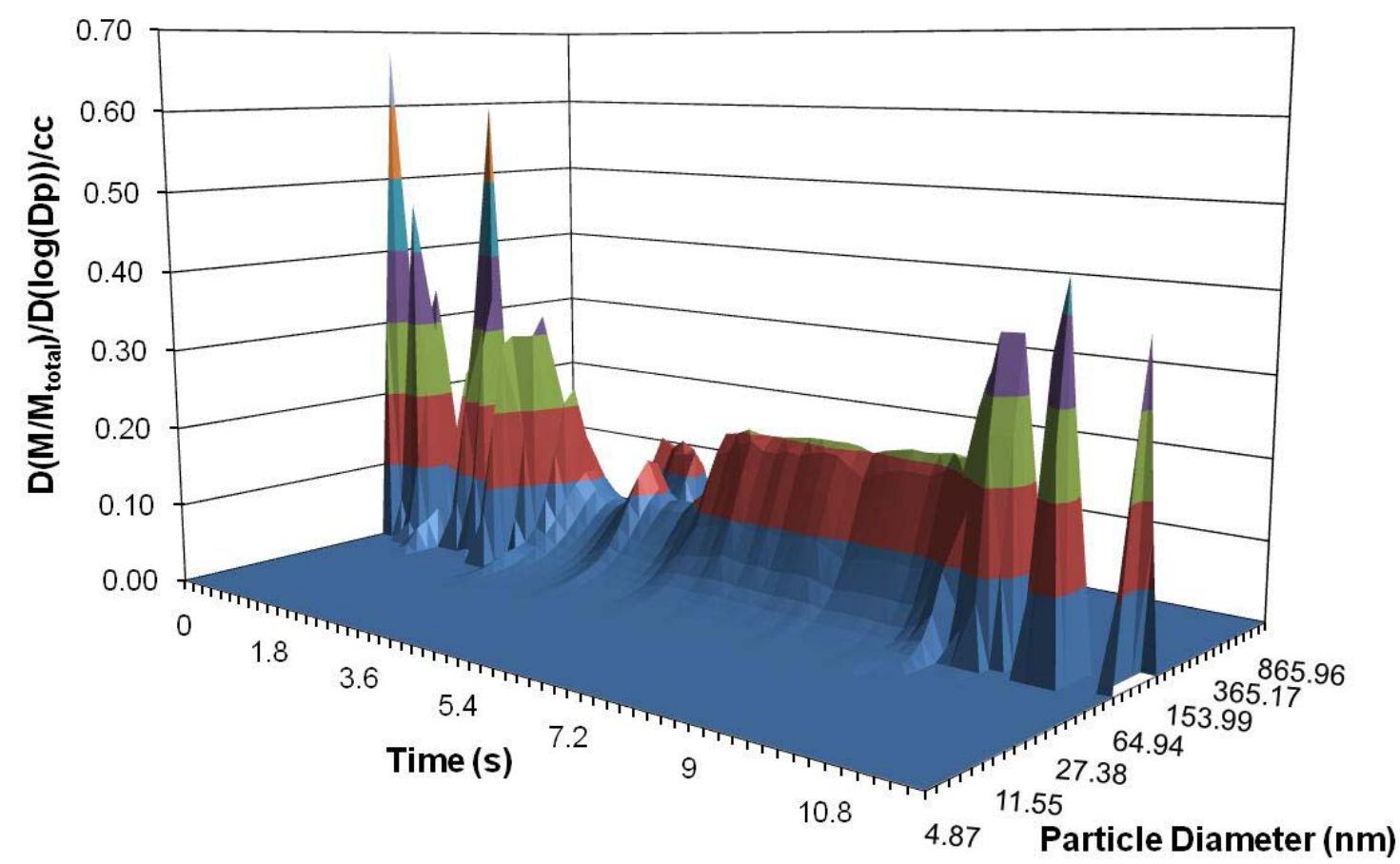

Figure 4-7: Normalized mass weighted particle size distribution obtained with the diesel vehicle.

In Figure 4-7, it can be seen that the mass weighted size distribution during the diesel drive-by incident is dominated by a marginally wider accumulation mode than before and after the drive-by incident. This could be significant to exposure because most of the particles are just above the range typically associated with UFPs which have an alveolar deposition fraction of about 0.7 . 


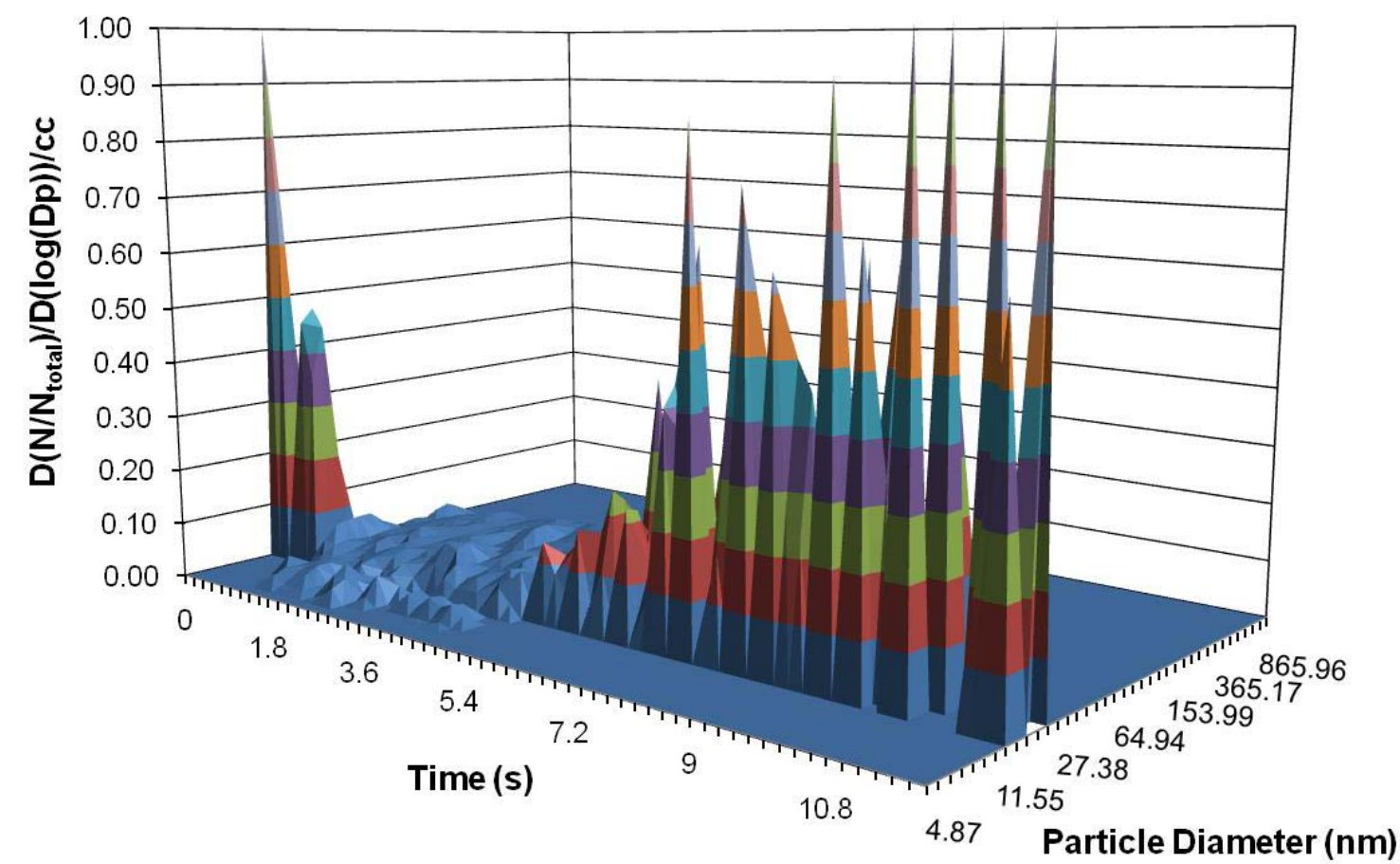

Figure 4-8: Normalized number weighted particle size distribution obtained with the gasoline vehicle.

In Figure 4-8, it can be seen that before and after the gasoline drive-by incident the normalized number weighted particle size distribution is dominated by the smaller accumulation mode particles. However, during the drive-by incident (about 2 to 4 seconds) the normalized number weighted size distribution is composed of a combination of accumulation mode and nuclei mode particles. 


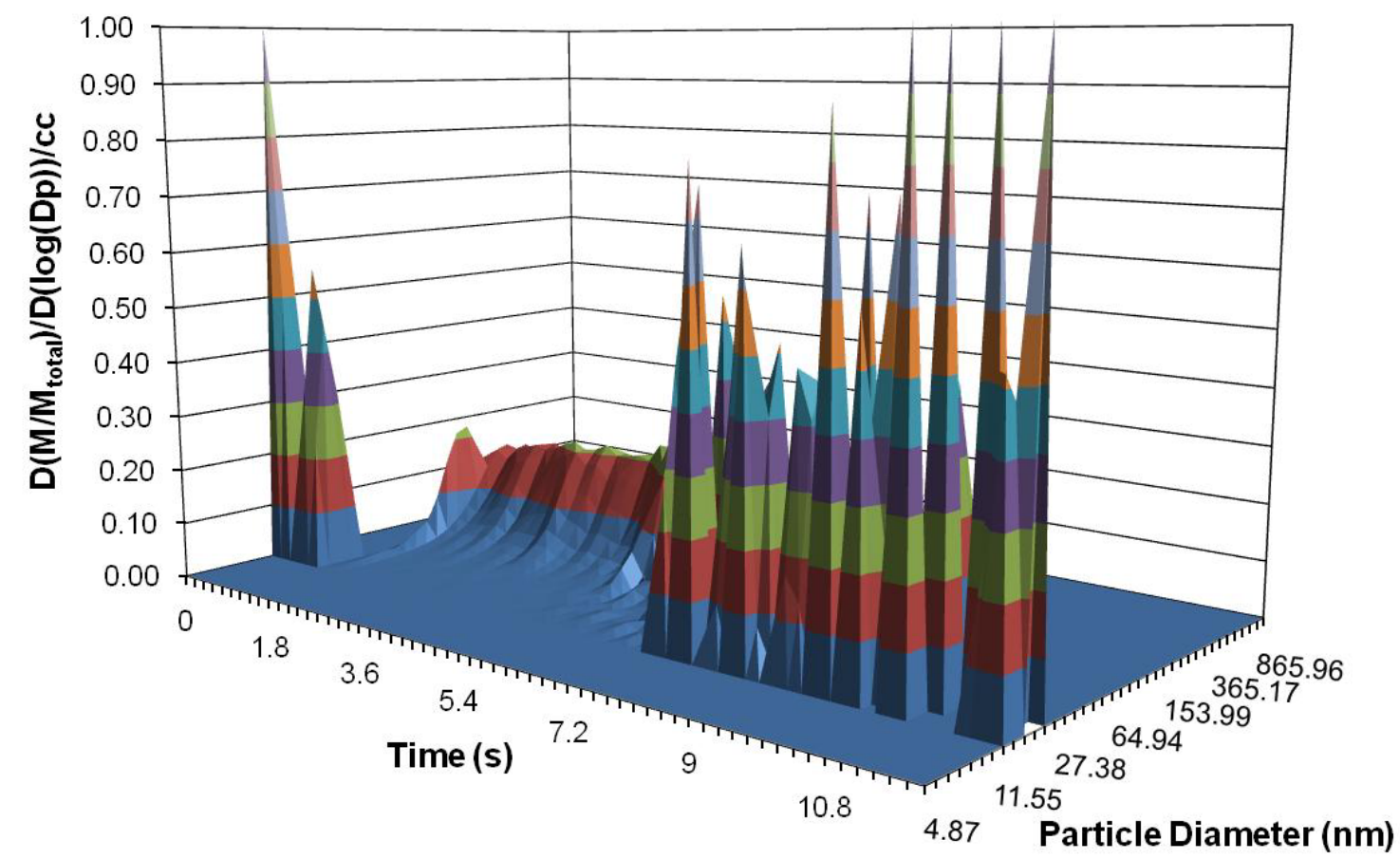

Figure 4-9: Normalized mass weighted particle size distribution obtained with the gasoline vehicle.

In Figure 4-9, the normalized mass weighted particle size distribution obtained during the gasoline vehicle drive-by incident is similar to that of the diesel vehicle in that it is also dominated by accumulation mode particles. However, before and after the main plume from the incident is observed, the normalized mass weighted particle size distribution is dominated by smaller accumulation mode particles. Although the normalized number and mass weighted particle size distributions during full load acceleration tests with both the diesel and gasoline vehicle are similar, the nonnormalized particle size distributions are not. The differences can be seen in Figure 4-10, Figure 4-11, Figure 4-12, and Figure 4-13. For contrast, the lognormal particle size distributions for both vehicles for all scenarios during each test run can be seen in Appendix D. From the figures in Appendix D, it can be seen that the particle counts obtained with the diesel vehicle are typically an order of magnitude greater those obtained with the gasoline vehicle. 


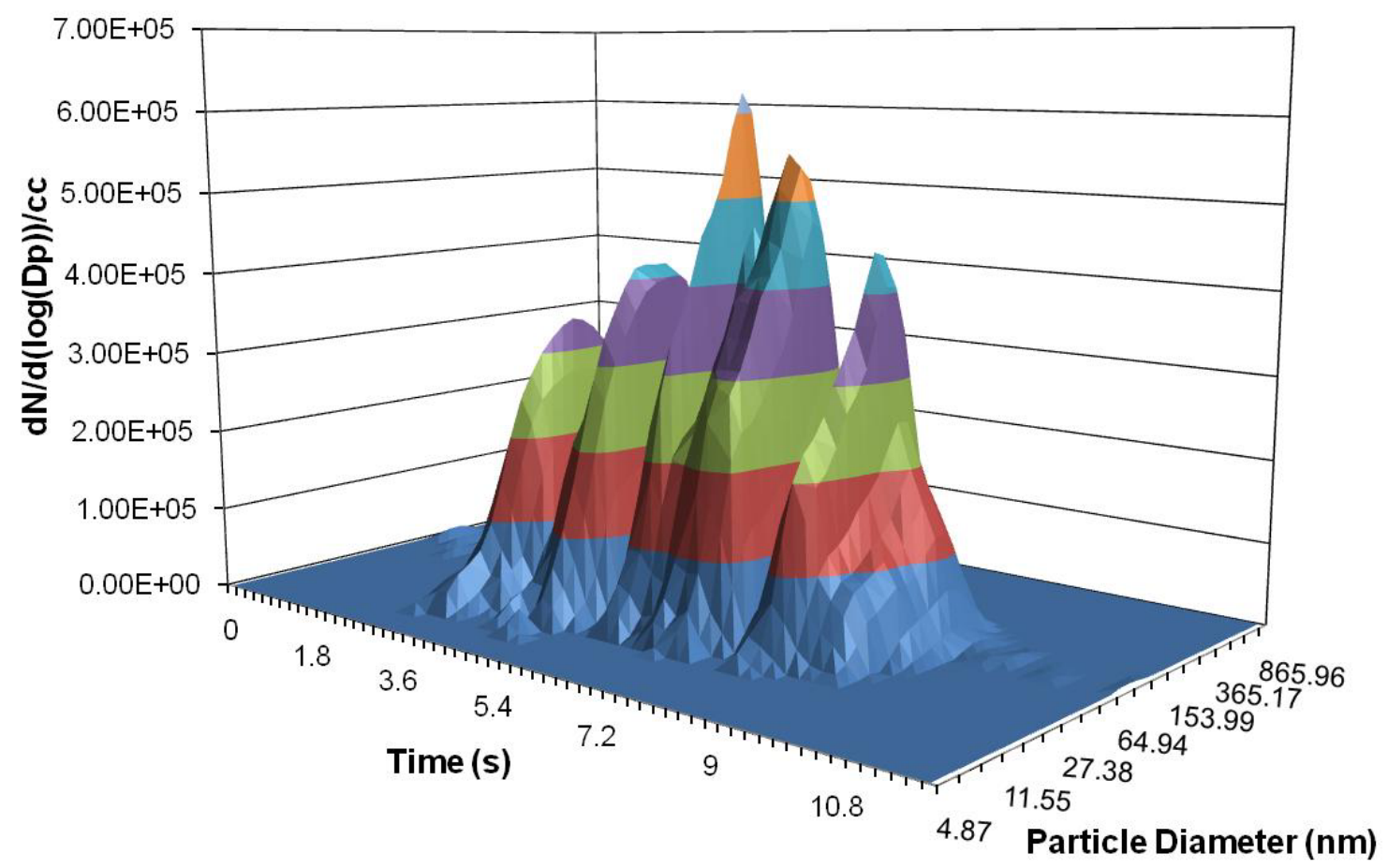

Figure 4-10: Number weighted particle size distribution obtained with the diesel vehicle.

In Figure 4-10, the number weighted size distribution obtained with the diesel vehicle during a full load acceleration test reached a maximum of about 600,000 particles/cc. However, note that this maximum is one of the lowest observed during the diesel drive-by acceleration tests. This maximum occurs at a particle diameter of about 86 $\mathrm{nm}$ which definitely lies within the accumulation mode. 


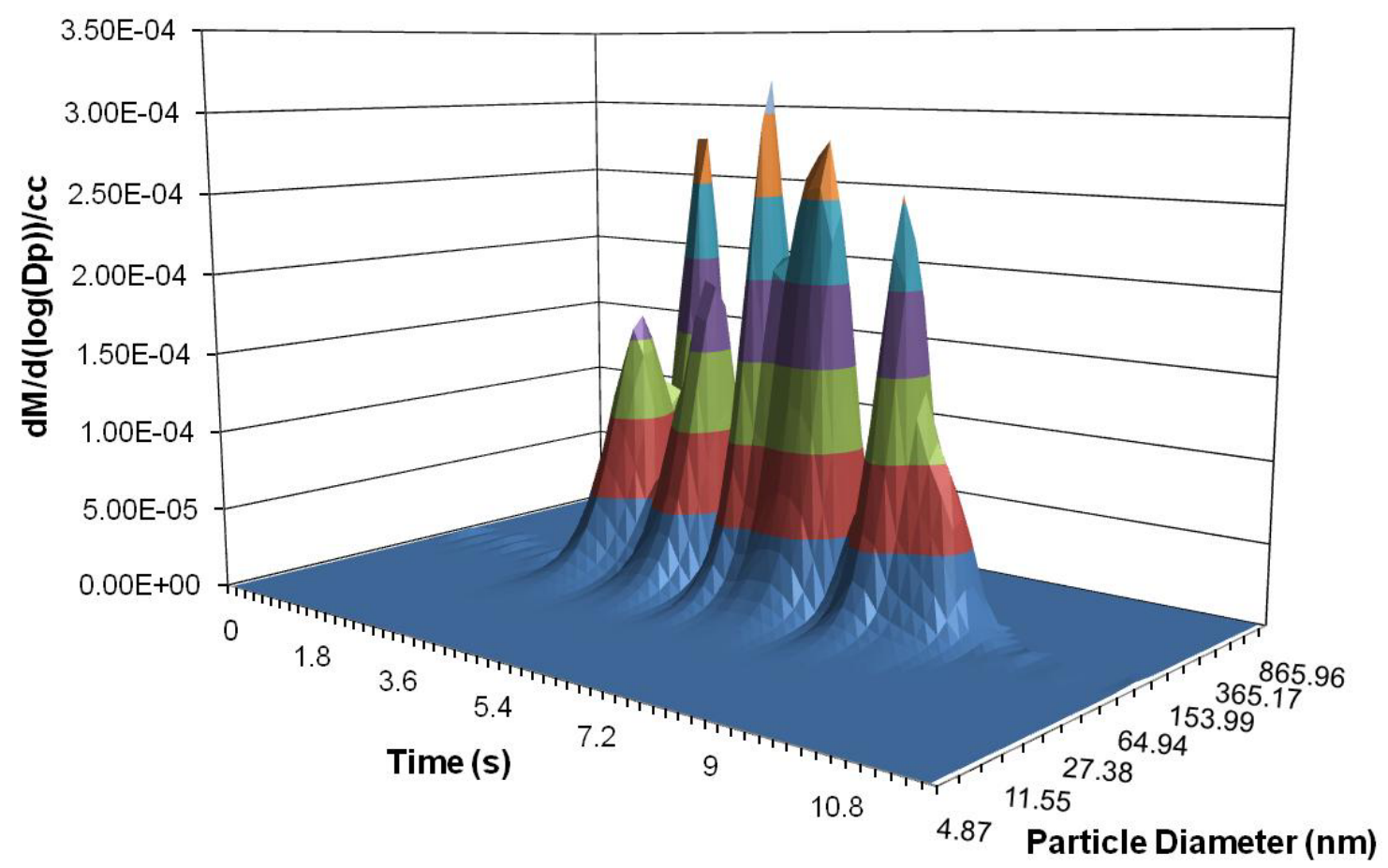

Figure 4-11: Mass weighted particle size distribution obtained with the diesel vehicle.

In Figure 4-11, the mass weighted particle size distribution obtained with the diesel vehicle during a full load acceleration test reached a maximum of about 0.0003 $\mu \mathrm{g} / \mathrm{cc}$. Again, note that this maximum is one of the lowest observed during the diesel drive-by acceleration tests. Although the maximum particle concentration had a particle diameter of around $86 \mathrm{~nm}$, the maximum of the mass weighted distribution had a particle diameter of about $133 \mathrm{~nm}$ which also lies within the accumulation mode. 


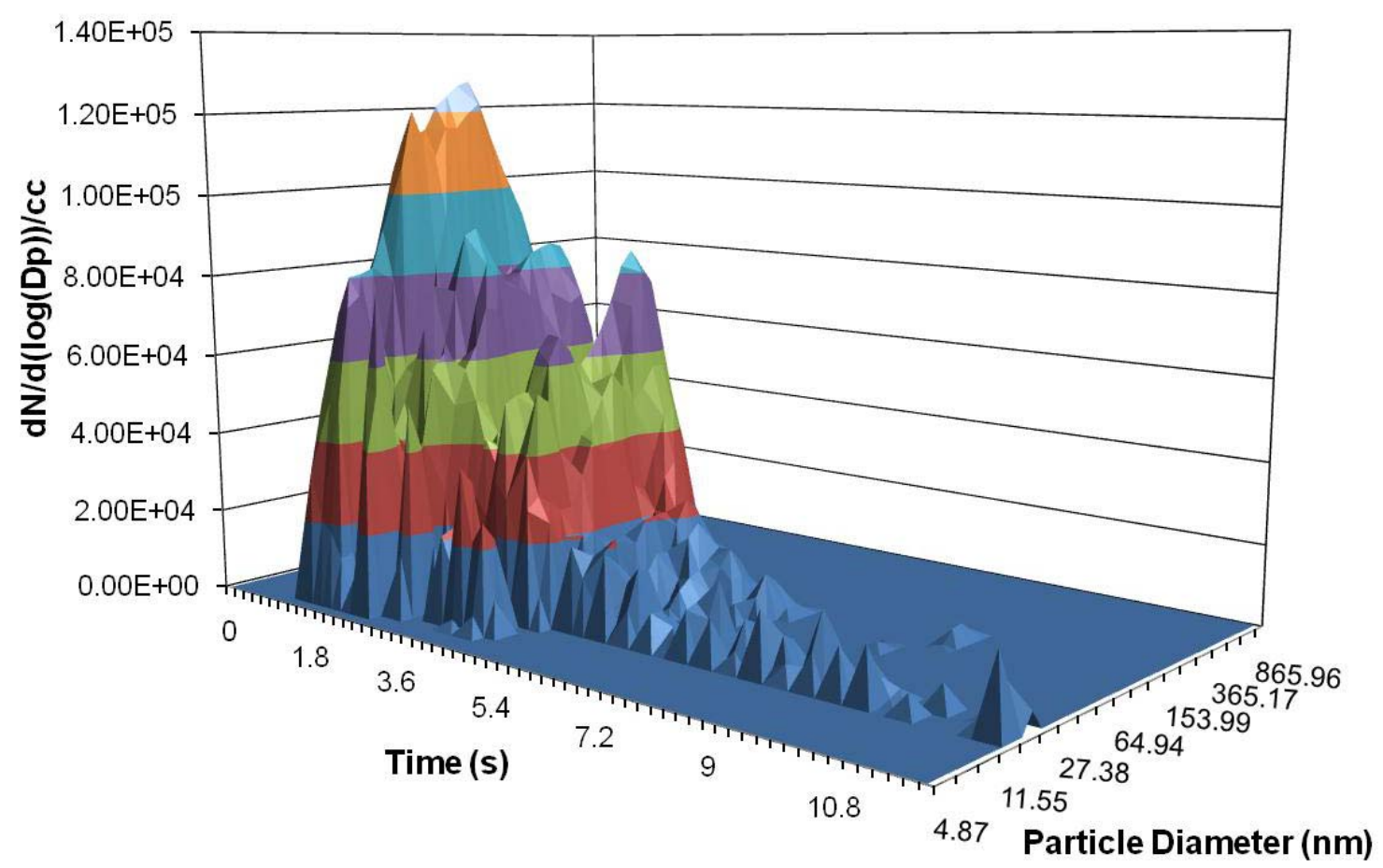

Figure 4-12: Number weighted particle size distribution obtained with the gasoline vehicle.

In Figure 4-12, the number weighted size distribution obtained with the gasoline vehicle during a full load acceleration test reached a maximum of about 128,000 particles/cc. Although this particle concentration is a maximum of the gasoline vehicle tests, it is four times lower than the lowest maximum of the diesel vehicle tests. However, while the particle concentrations of the gasoline vehicle are lower, the size distribution obtained with the gasoline vehicle is comprised of nuclei and accumulation mode particles which is smaller than the size distribution from the diesel vehicle which is comprised primarily of accumulation mode particles. The highest number of particles occurs in particles having diameters around $48.7 \mathrm{~nm}$. 


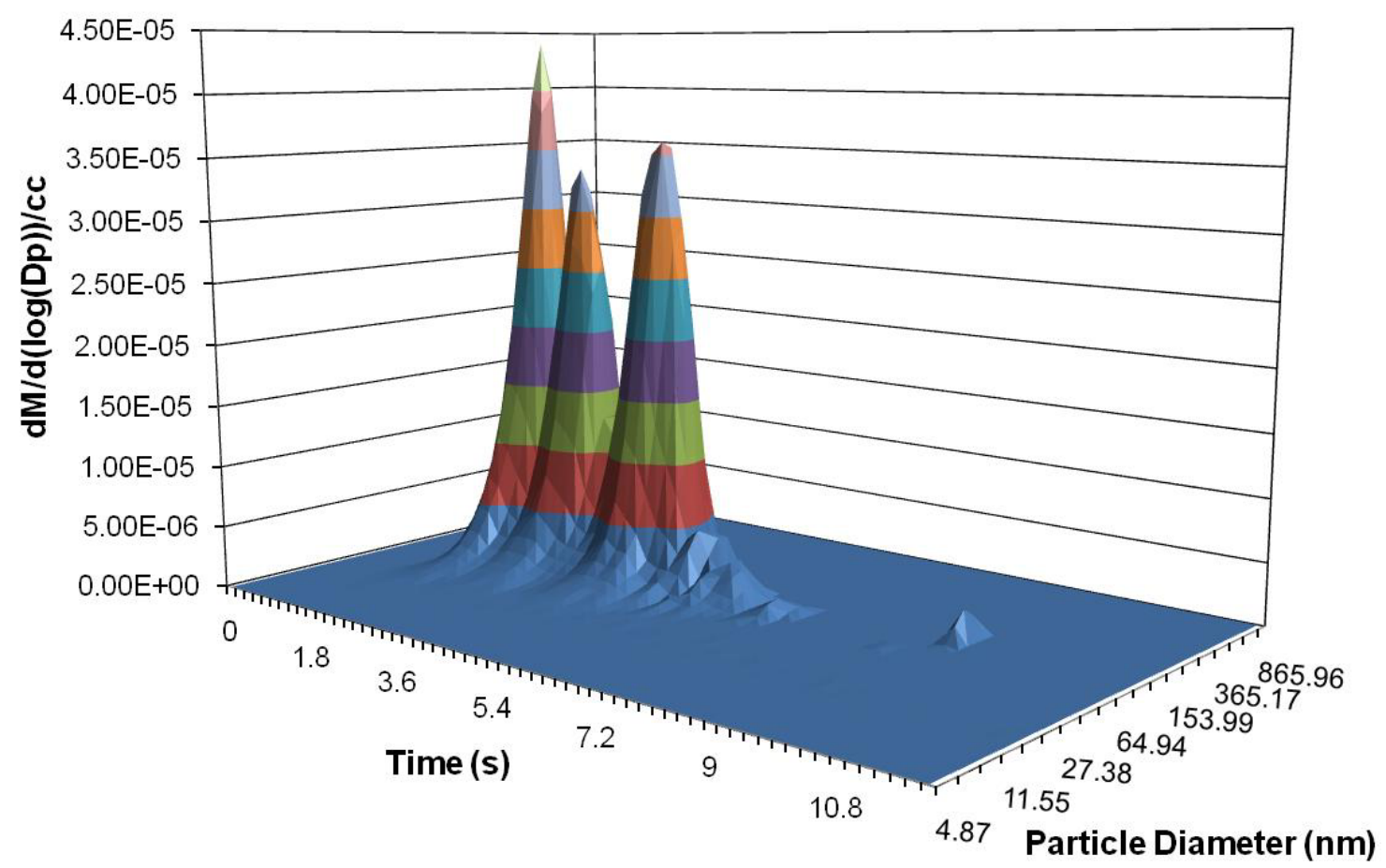

Figure 4-13: Mass weighted particle size distribution obtained with the gasoline vehicle.

In Figure 4-13, the mass weighted particle size distribution obtained with the gasoline vehicle during a full load acceleration test reached a maximum of about $0.000044 \mu \mathrm{g} / \mathrm{cc}$. Although this mass concentration is a maximum of the gasoline vehicle tests, it is almost seven times lower than the lowest maximum of the diesel vehicle tests. However, unlike with the particle concentrations, the mass concentrations obtained with the gasoline and diesel vehicles have similar size distributions which are comprised primarily of accumulation mode particles. The largest amount of particle mass occurs in particles having diameters around $133.35 \mathrm{~nm}$.

The average incident particulate matter concentrations observed and the corresponding incident durations for the hard acceleration, medium acceleration, and cruising tests with the diesel test vehicle can be seen in Table 4-1, Table 4-2, and Table 4-3, respectively. The average incident particulate matter concentrations observed and the corresponding incident durations for the hard acceleration, medium acceleration, and cruising tests with the gasoline test vehicle can be seen in, Table 4-5, and Table 4-6, respectively. In these tables, the durations were determined mathematically by the methods described previously and the particulate matter concentrations were computed 
using the DMS specific method. Because the hard acceleration tests are of much more concern due to the amount of PM discharged, the results from Table 4-3 and Table 4-4 are shown visually in Figure 4-14 and Figure 4-15.

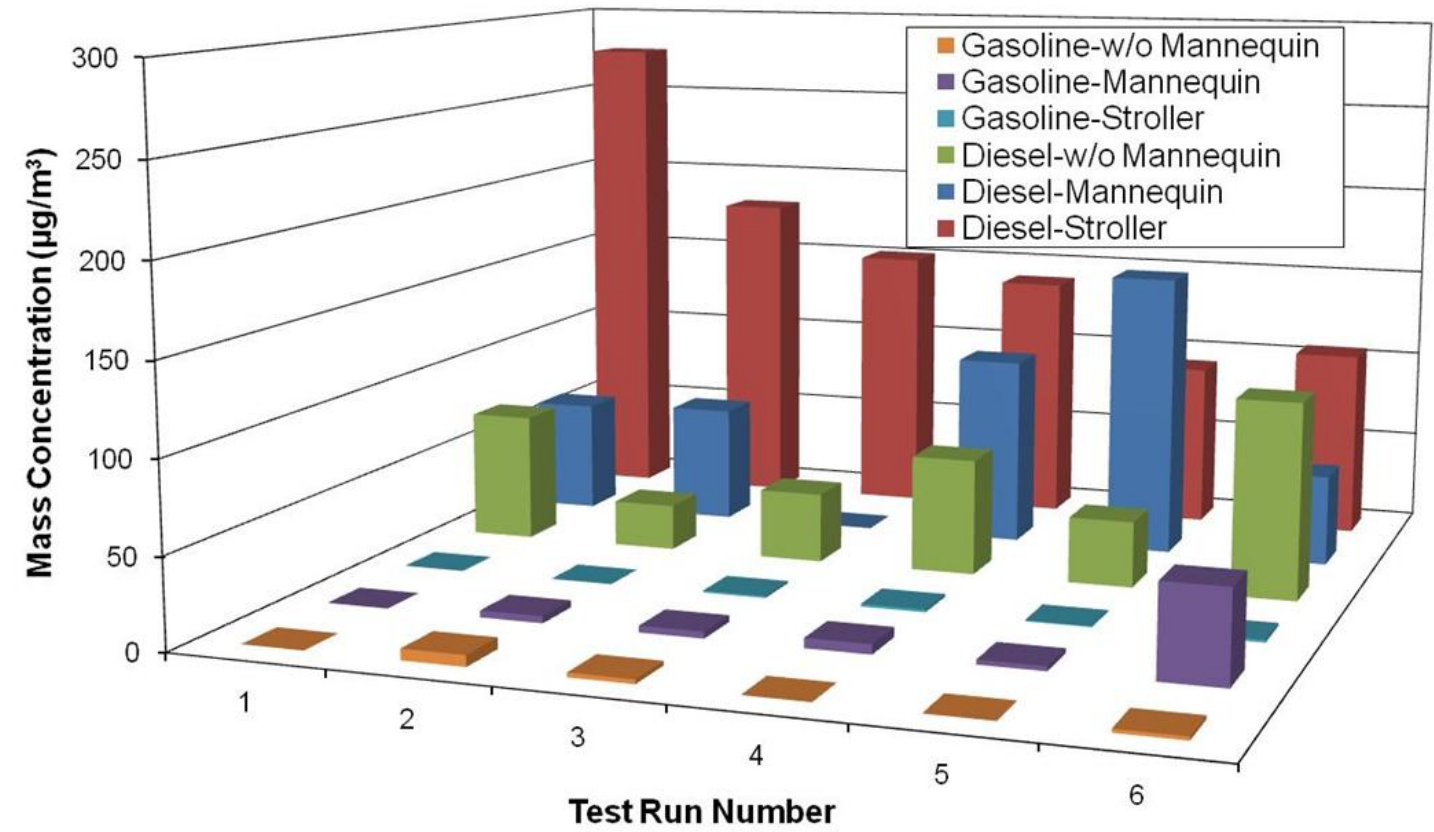

Figure 4-14: Average incident PM concentrations for all full load acceleration tests.

Figure 4-14 shows the comparison between the gasoline and diesel test vehicles on a larger scale. In this figure, the average incident PM concentrations for each test run and each scenario can be seen. Except for a few exceptions (the anomaly in test run 6 for the gasoline-mannequin case), it is obvious that the PM concentrations obtained with the diesel vehicle are orders of magnitude greater than those obtained with the gasoline vehicle. Additionally, it can be seen that within the diesel test runs, the child exposure scenario referred to as the "stroller" scenario has higher concentrations than the other exposure scenarios considered. This has implications about where exposure is greatest (notice the drive-by diagram shown in Figure 3-7). This figure also shows that the average incident PM concentrations obtained with the diesel vehicle can approach the same order of magnitude as concentrations used in human dose studies. It must be kept in mind though, that current in-use diesel vehicles could potentially produce PM 
concentrations that are an order of magnitude or more, greater than those obtained in this study.

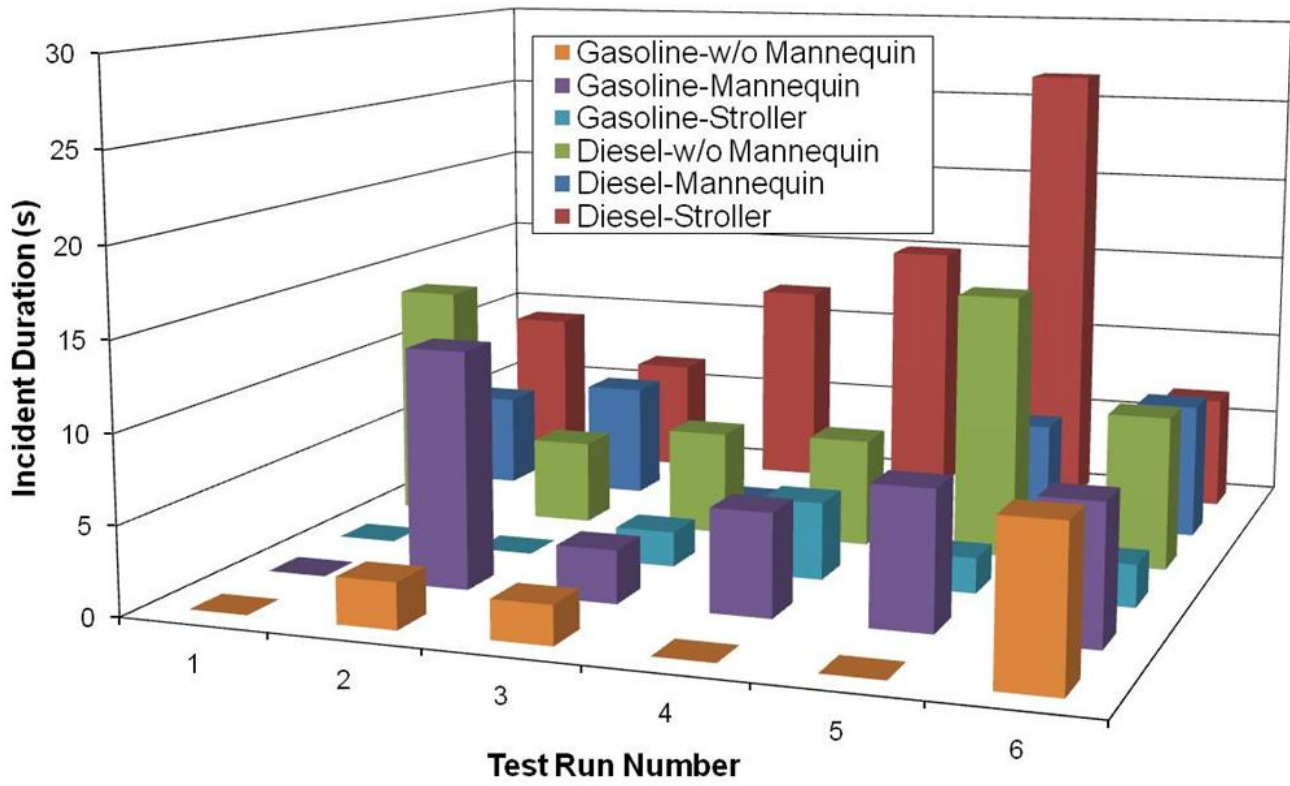

Figure 4-15: Average incident durations for all full load acceleration tests.

In Figure 4-15, the average incident durations associated with the concentrations shown in Figure 4-14 are shown. Although the durations appear to be random, in general, the durations of the diesel drive-by incidents are longer. However, because of numerous factors affecting these results, they are inconclusive. 
Table 4-1: Average incident PM concentrations, durations, and peak concentrations for hard acceleration diesel drive-bys.

\begin{tabular}{|l|c|c|c|c|}
\hline Scenario & Run & $\begin{array}{c}\text { Average Incident } \\
\text { Concentration }\left(\boldsymbol{\mu g} / \mathbf{m}^{\mathbf{3}}\right)\end{array}$ & $\begin{array}{c}\text { Incident Duration } \\
(\mathbf{s})\end{array}$ & $\begin{array}{c}\text { Peak Concentration } \\
\left(\boldsymbol{\mu} \mathbf{g} / \mathbf{m}^{\mathbf{3}}\right)\end{array}$ \\
\hline Mannequin & 1 & 62.95 & 5.4 & 267.10 \\
\hline & 2 & 65.66 & 6.6 & 136.44 \\
\hline & 3 & 0.00 & 0 & 0.00 \\
\hline & 4 & 105.75 & 1.4 & 427.31 \\
\hline & 5 & 159.07 & 6 & 554.03 \\
\hline & 6 & 50.64 & 7.8 & 164.65 \\
\hline Stroller & 1 & 276.26 & 9.2 & 1338.97 \\
\hline & 2 & 180.31 & 6.6 & 814.23 \\
\hline & 3 & 151.22 & 12 & 1297.43 \\
\hline & 4 & 139.77 & 15 & 821.56 \\
\hline & 5 & 92.26 & 26.6 & 975.70 \\
\hline & 6 & 105.52 & 6.6 & 322.31 \\
\hline w/o Mannequin & 1 & 71.67 & 13.6 & 340.31 \\
\hline & 2 & 25.52 & 4.8 & 170.13 \\
\hline & 3 & 38.89 & 6 & 207.42 \\
\hline & 4 & 64.85 & 6.2 & 183.65 \\
\hline & 5 & 36.82 & 15.2 & 220.37 \\
\hline & 6 & 109.20 & 8.8 & 263.16 \\
\hline
\end{tabular}

The results from Table 4-1 imply that average incident particulate matter concentrations near a roadway during a drive-by incident with the diesel test vehicle under heavy acceleration (100\% load) are near levels used in human clinical studies (e.g. $300 \mu \mathrm{g} / \mathrm{m}^{3}$ ). Furthermore, the peak concentrations observed during these drive-by incidents can reach more than 4 times those concentrations used in human studies. Low particulate concentrations such as these have been documented to cause acute health effects including accumulation of particulate matter in the lungs. However, accumulation occurs over longer periods of time or numerous exposure incidents. It must be considered, though, that these results were obtained via a simplistic model that represents an in-use minimum for medium and heavy duty diesel vehicle PM expulsion. In reality, a large number of drive-bys would have a more significant effect on exposure because ambient levels of pollutants would build as not all of the pollutants are carried away or dispersed. Additionally, it must be noted that the study was not attempting to map the exhaust plumes and as such, measurements were not necessarily taken at the center of the exhaust plume. This implies that particulate matter concentrations could be significantly higher. Also, note that during the full load acceleration tests, the child exposure scenario experienced the highest PM concentrations which were almost 2 and 3 times higher than the mannequin and no mannequin cases. Furthermore, the mannequin case had concentrations which were $50 \%$ higher than the no mannequin case. 
Table 4-2: Average incident PM concentrations, durations, and peak concentrations for medium acceleration diesel drive-bys.

\begin{tabular}{|l|c|c|c|c|}
\hline Scenario & Run & $\begin{array}{c}\text { Average Incident } \\
\text { Concentration }\left(\boldsymbol{\mu g} / \mathbf{m}^{\mathbf{3}}\right)\end{array}$ & $\begin{array}{c}\text { Incident Duration } \\
(\mathbf{s})\end{array}$ & $\begin{array}{c}\text { Peak Concentration } \\
\left(\boldsymbol{\mu g} / \mathbf{m}^{\mathbf{3}}\right)\end{array}$ \\
\hline Mannequin & 1 & 29.22 & 5 & 57.77 \\
\hline & 2 & 39.37 & 4.6 & 137.08 \\
\hline & 3 & 19.82 & 2 & 96.78 \\
\hline & 4 & 0.00 & 0 & 0.00 \\
\hline & 5 & 18.97 & 7.2 & 125.04 \\
\hline & 6 & 10.94 & 10.4 & 79.60 \\
\hline Stroller & 1 & 19.63 & 2.2 & 397.78 \\
\hline & 2 & 3.38 & 0.4 & 206.12 \\
\hline & 3 & 0.00 & 0 & 123.18 \\
\hline & 4 & 16.93 & 4 & 27.97 \\
\hline & 5 & 0.00 & 0 & 0.00 \\
\hline & 6 & 0.00 & 0 & 116.05 \\
\hline w/o Mannequin & 1 & 40.06 & 11.6 & 43.89 \\
\hline & 2 & 46.92 & 8.6 & 4.45 \\
\hline & 3 & 41.58 & 19.8 & 0.00 \\
\hline & 4 & 7.17 & 10.6 & 66.22 \\
\hline & 5 & 0.00 & 0 & 0.00 \\
\hline & 6 & 57.74 & 3.4 & 0.00 \\
\hline
\end{tabular}

It can be seen in Table 4-2 that the average incident PM concentrations obtained during part load acceleration tests with the diesel vehicle are lower than those obtained during full load acceleration tests with the diesel vehicle. Similarly, the peak concentrations observed are much lower as well. These average concentrations are lower than those used in human dose studies, but these peak concentrations still approach those used in human dose studies. Additionally, note that there were many incidents with average concentrations of zero implying background level measurements. Also, note that during the part load acceleration tests, the without mannequin case observed $60 \%$ higher exposure than the mannequin scenario, and both were at least $70 \%$ higher than the stroller case. This result is opposite from the result obtained during the hard acceleration tests. These results do make sense, though, because the exhaust flow rate was less during medium acceleration, it was more likely that the buoyancy of the exhaust carried it up before it reached the child. Consequently, the adult experienced a greater exposure. Similarly, during hard acceleration tests, the exhaust flow rate was high enough to push the exhaust to the point of the baby stroller before buoyancy caused it to rise. These results imply that exposure is dependent on location with respect to the passing vehicle's tailpipe as well as the operating conditions of the vehicle. 
Table 4-3: Average incident PM concentrations, durations, and peak concentrations for cruising diesel drive-bys.

\begin{tabular}{|l|c|c|c|c|}
\hline Scenario & Run & $\begin{array}{c}\text { Average Incident } \\
\text { Concentration }\left(\boldsymbol{\mu g} / \mathbf{m}^{\mathbf{3}}\right)\end{array}$ & $\begin{array}{c}\text { Incident Duration } \\
(\mathbf{s})\end{array}$ & $\begin{array}{c}\text { Peak Concentration } \\
\left(\boldsymbol{\mu g} / \mathbf{m}^{\mathbf{3}}\right)\end{array}$ \\
\hline Mannequin & 1 & 9.60 & 16.6 & 60.32 \\
\hline & 2 & 3.82 & 1.6 & 6.41 \\
\hline & 3 & 11.82 & 0.6 & 15.98 \\
\hline & 4 & 21.62 & 1.6 & 29.62 \\
\hline & 5 & 5.79 & 4.4 & 20.97 \\
\hline & 6 & 3.02 & 5.8 & 7.13 \\
\hline & 1 & 15.66 & 8 & 283.44 \\
\hline & 2 & 16.73 & 1.4 & 262.00 \\
\hline & 3 & 12.48 & 15.2 & 70.91 \\
\hline & 4 & 0.00 & 0 & 126.62 \\
\hline & 5 & 15.38 & 9.6 & 36.69 \\
\hline & 6 & 10.02 & 12.4 & 42.21 \\
\hline & 1 & 23.20 & 15 & 60.32 \\
\hline & 2 & 149.59 & 2 & 6.41 \\
\hline & 3 & 37.46 & 3.4 & 15.98 \\
\hline & 4 & 13.13 & 17.6 & 29.62 \\
\hline & 5 & 5.78 & 18 & 20.97 \\
\hline & 6 & 11.57 & 8.4 & 7.13 \\
\hline
\end{tabular}

Table 4-3 shows the average incident PM concentrations and durations from the diesel vehicle cruising tests. It can be seen that the average and peak concentrations are typically even lower than those observed during part load acceleration tests, and consequently, are lower than those concentrations used in human dose studies. However, the peak concentrations do approach the same order of magnitude as those used in human dose studies. Additionally, during the cruising tests, there were fewer "zero" incidents where PM concentrations did not increase above background levels. During the cruising drive-by tests, the highest PM concentrations were observed for the without mannequin tests which were 30\% higher than the stroller case and $96 \%$ higher than the mannequin case. These results exclude the abnormal concentrations such as observed in test run 2 for the without mannequin case. 
Table 4-4: Average incident PM concentrations, durations, and peak concentrations for hard acceleration gasoline drive-bys.

\begin{tabular}{|l|c|c|c|c|}
\hline Scenario & Run & $\begin{array}{c}\text { Average Incident } \\
\text { Concentration }\left(\boldsymbol{\mu g} / \mathbf{m}^{\mathbf{3}}\right)\end{array}$ & $\begin{array}{c}\text { Incident Duration } \\
(\mathbf{s})\end{array}$ & $\begin{array}{c}\text { Peak Concentration } \\
\left(\boldsymbol{\mu g} / \mathbf{m}^{\mathbf{3}}\right)\end{array}$ \\
\hline Mannequin & 1 & 0.00 & 0 & 0.00 \\
\hline & 2 & 3.81 & 13.4 & 17.67 \\
\hline & 3 & 3.63 & 3 & 11.62 \\
\hline & 4 & 5.45 & 5.8 & 16.15 \\
\hline & 5 & 2.57 & 7.8 & 6.23 \\
\hline & 6 & 50.64 & 7.8 & 0.00 \\
\hline Stroller & 1 & 0.00 & 0 & 0.00 \\
\hline & 2 & 0.00 & 0 & 0.00 \\
\hline & 3 & 1.05 & 2 & 2.10 \\
\hline & 4 & 1.45 & 4.4 & 7.74 \\
\hline & 5 & 0.44 & 2 & 0.79 \\
\hline & 6 & 1.48 & 2.4 & 5.81 \\
\hline w/o Mannequin & 1 & 0.00 & 0 & 0.00 \\
\hline & 2 & 6.14 & 2.6 & 12.12 \\
\hline & 3 & 2.42 & 2.2 & 4.66 \\
\hline & 4 & 0.00 & 0 & 0.00 \\
\hline & 5 & 0.00 & 0 & 0.00 \\
\hline & 6 & 1.90 & 8.8 & 3.57 \\
\hline
\end{tabular}

In Table 4-4, the average incident PM concentrations and durations obtained during the full load acceleration gasoline vehicle drive-by tests are shown. Except for an occasional anomaly, it can be seen that these average and peak concentrations are orders of magnitude lower than those obtained with the diesel vehicle. This implies that these concentrations are less likely to cause acute health effects. Also, excluding the abnormally high concentration in test run 6 of the mannequin case, the concentrations are too low to determine which scenario experienced a higher exposure. 
Table 4-5: Average incident PM concentration, durations, and peak concentrations for medium acceleration gasoline drive-bys.

\begin{tabular}{|l|c|c|c|c|}
\hline Scenario & Run & $\begin{array}{c}\text { Average Incident } \\
\text { Concentration }\left(\boldsymbol{\mu g} / \mathbf{m}^{\mathbf{3}}\right)\end{array}$ & $\begin{array}{c}\text { Incident Duration } \\
(\mathbf{s})\end{array}$ & $\begin{array}{c}\text { Peak Concentration } \\
\left(\boldsymbol{\mu g} / \mathbf{m}^{\mathbf{3}}\right)\end{array}$ \\
\hline Mannequin & 1 & 0.00 & 0 & 0.00 \\
\hline & 2 & 0.00 & 0 & 0.00 \\
\hline & 3 & 0.00 & 0 & 0.00 \\
\hline & 4 & 0.00 & 0 & 0.00 \\
\hline & 5 & 0.00 & 0 & 0.00 \\
\hline & 6 & 0.00 & 0 & 0.00 \\
\hline Stroller & 1 & 0.55 & 0.4 & 1.85 \\
\hline & 2 & 0.00 & 0 & 0.00 \\
\hline & 3 & 0.00 & 0 & 0.00 \\
\hline & 4 & 0.00 & 0 & 0.00 \\
\hline & 5 & 0.00 & 0 & 0.00 \\
\hline & 6 & 0.49 & 1.6 & 0.00 \\
\hline w/o Mannequin & 1 & 0.68 & 0.6 & 1.06 \\
\hline & 2 & 0.00 & 0 & 0.00 \\
\hline & 3 & 0.00 & 0 & 0.00 \\
\hline & 4 & 0.00 & 0 & 0.00 \\
\hline & 5 & 0.00 & 0 & 0.00 \\
\hline & 6 & 0.00 & 0 & 1.16 \\
\hline
\end{tabular}

In Table 4-5, the average incident PM concentrations and durations obtained during the part load acceleration gasoline vehicle drive-by tests are shown. It can be seen that the average and peak concentrations are all nearly zero implying no increase above background concentrations. Because nearly all of the average and peak incident concentrations are zero, it is difficult to judge which if any exposure scenario experienced a higher exposure. 
Table 4-6: Average incident PM concentrations, durations, and peak concentrations for cruising gasoline drive-bys.

\begin{tabular}{|l|c|c|c|c|}
\hline Scenario & Run & $\begin{array}{c}\text { Average Incident } \\
\text { Concentration }\left(\boldsymbol{\mu g} / \mathbf{m}^{\mathbf{3}}\right)\end{array}$ & $\begin{array}{c}\text { Incident Duration } \\
(\mathbf{s})\end{array}$ & $\begin{array}{c}\text { Peak Concentration } \\
\left(\boldsymbol{\mu g} / \mathbf{m}^{\mathbf{3}}\right)\end{array}$ \\
\hline Mannequin & 1 & 0.00 & 0 & 0.00 \\
\hline & 2 & 0.00 & 0 & 0.00 \\
\hline & 3 & 0.97 & 8.6 & 20.07 \\
\hline & 4 & 0.00 & 0 & 0.00 \\
\hline & 5 & 0.00 & 0 & 0.00 \\
\hline & 6 & 0.00 & 0 & 0.00 \\
\hline & 1 & 0.00 & 0 & 0.00 \\
\hline & 2 & 0.00 & 0 & 0.00 \\
\hline & 3 & 0.00 & 0 & 0.00 \\
\hline & 4 & 0.00 & 0 & 0.00 \\
\hline & 5 & 0.00 & 0 & 0.00 \\
\hline & 6 & 0.00 & 0 & 0.00 \\
\hline & 1 & 0.00 & 0 & 0.00 \\
\hline & 2 & 0.00 & 0 & 0.00 \\
\hline & 3 & 0.00 & 0 & 0.00 \\
\hline & 4 & 0.00 & 0 & 0.00 \\
\hline & 5 & 0.00 & 0 & 0.00 \\
\hline & 6 & 0.00 & 0 & 0.00 \\
\hline
\end{tabular}

In Table 4-6, the average incident PM concentrations and durations obtained during the cruising gasoline vehicle drive-by tests are shown. It can be seen that the average and peak concentrations are all nearly zero implying no increase above background concentrations. Because nearly all of the average incident concentrations are zero, it is difficult to judge which if any exposure scenario experienced a higher exposure.

As expected, the average incident particulate matter concentrations for the medium acceleration (50\% load) and cruising scenarios are lower than when the vehicle accelerated at nearly $100 \%$ load. These average concentrations while lower were still high enough to cause acute health effects over a long period of time (many hours or exposures). This occurrence is also observed for the gasoline tests. However, the average incident particulate matter concentrations for the acceleration at 50\% load and cruising scenarios with the gasoline vehicle were either zero or equivalent to background levels. These results again show that the diesel test vehicle typically emits orders of magnitude more particulate matter than the gasoline test vehicle.

The average particulate concentrations measured throughout the full load acceleration tests with the diesel test vehicle were 74.01 and $157.56 \mu \mathrm{g} / \mathrm{m}^{3}$ for the adult pedestrian and child in stroller scenarios, respectively. The average duration of these incidents was determined to be approximately 4.5 and 12.67 seconds, respectively. The 
average particulate concentrations measured throughout the full load acceleration tests with the gasoline test vehicle were 3.09 and $0.74 \mu \mathrm{g} / \mathrm{m}^{3}$ for the adult pedestrian and child in stroller scenarios, respectively. The average duration of these incidents was determined to be approximately 6.3 and 1.8 seconds, respectively. These concentrations which represent the average of the incident average concentrations were used to simulate exposure which will be discussed below.

The maximum concentrations averaged over 1.5 seconds to simulate a poorly timed breath observed for the adult pedestrian and child in stroller scenarios during a drive-by with the diesel test vehicle were 370.39 and $877.1 \mu \mathrm{g} / \mathrm{m}^{3}$, respectively. The maximum concentrations averaged over 1.5 seconds to simulate a poorly timed breath observed for the adult pedestrian and child in stroller scenarios during a drive-by with the gasoline test vehicle were 14.19 and $2.78 \mu \mathrm{g} / \mathrm{m}^{3}$, respectively. The maximum concentrations averaged over 2.5 seconds for a similar breath observed for the adult pedestrian and child in stroller scenarios during a drive-by with the diesel test vehicle were 297.92 and $663.84 \mu \mathrm{g} / \mathrm{m}^{3}$, respectively. The maximum concentrations averaged over 2.5 seconds for a similar breath observed for the adult pedestrian and child in stroller scenarios during a drive-by with the gasoline test vehicle were 11.64 and $2.26 \mu \mathrm{g} / \mathrm{m}^{3}$, respectively.

While the particulate matter concentrations averaged over incident durations for both test vehicles are lower than the concentrations used in many human clinical studies, the maximum concentrations observed for 1.5 and 2.5 seconds (i.e. the length of a breath) were as much as 3 times higher for the diesel test vehicle and as much as 40 times lower for the gasoline test vehicle. This result implies that particulate concentrations from diesel vehicle drive-by incidents can easily reach levels that cause acute health affects while gasoline vehicle drive-bys may not. However, the duration is still very small compared to the 1 or 2 hour exposures used in clinical studies. Although numerous particulate exposure studies have been conducted on humans [20-30], due to the difficulties faced in assessing the exposure and the corresponding health effects, there has not been enough information to develop standards that address such short exposures. Furthermore, there is not enough information regarding human dose studies to elicit a 
threshold concentration, beyond which health effects are certain to occur. More research is necessary in each of these areas.

Although the results from this study show that curbside particulate concentrations can easily surpass those used in clinical studies, the fact that the test vehicle is a new model vehicle with a catalytic converter must be taken into consideration. In-use fleet particulate matter emissions vary from vehicle to vehicle. For example, in the E-55/59 California truck emissions inventory program, medium duty trucks were exercised through a transient test cycle, termed MHDTLO, and it was found that a 1990 model year truck emitted particulate matter at a level that was 10.2 times higher than one model year 2000 truck, and 15.3 times higher than another model year 2000 truck [135]. A 1995 truck yielded particulate ratios of 4.5 and 6.3 relative to the two model year 2000 trucks [135]. The 2006 truck used in the present study had low mileage, and it is assumed that the particle emissions represent an in-use minimum for medium and heavy duty diesel vehicles prior to 2007 models with exhaust filtration. It is reasonable to believe that many in-use trucks would yield particulate levels substantially higher than those yielded by the 2006 diesel pickup.

\subsection{Exposure}

Breathing rates for men, women, and children both walking slowly and standing still, (see Table 4-7), were obtained from a study [136] produced by the California Air Resources Board and multiplied by the average incident concentrations. In this way, the mass of particulate matter inhaled per drive-by incident by men, women, and children could be computed. To see if the mass inhaled due to 264 drive-bys, a theoretical maximum computed previously, would be significant relative to the amount normally inhaled over a 24 hour period, the daily mass of particulate matter inhaled by pedestrians at different ambient concentrations was calculated. These values in addition to the values of mass inhaled due to 264 drive-bys with both vehicles by men, women, and children both walking and standing can be seen in Table 4-8. Figure 4-16 shows the results from Table 4-8 in a more visual form. It shows the percentage of mass inhaled daily corresponding to the mass inhaled due to 264 drive-by incidents. 
Table 4-7: Breathing rates for men, women, and children walking and standing [136].

\begin{tabular}{|c|c|c|c|}
\hline Activity & Adult Male & Adult Female & Children \\
\hline Walking & 24 & 20 & 14 \\
\hline Standing & 11 & 8 & 7 \\
\hline
\end{tabular}

Table 4-8: Mass inhaled due to 264 average drive-by incidents and daily at different ambient conditions.

\begin{tabular}{|c|c|c|c|c|c|c|c|c|}
\hline \multirow{2}{*}{$\begin{array}{l}\text { Scenario } \\
\text { Mannequin }\end{array}$} & \multicolumn{2}{|c|}{ Diesel Drive-by } & \multicolumn{2}{|c|}{ Gasoline Drive-by } & \multicolumn{2}{|c|}{ NAAQS } & \multicolumn{2}{|c|}{ Darrington, WA } \\
\hline & $\begin{array}{l}\text { Adult } \\
\text { Male }\end{array}$ & $\begin{array}{c}\text { Adult } \\
\text { Female }\end{array}$ & $\begin{array}{l}\text { Adult } \\
\text { Male }\end{array}$ & $\begin{array}{l}\text { Adult } \\
\text { Female }\end{array}$ & $\begin{array}{l}\text { Adult } \\
\text { Male }\end{array}$ & $\begin{array}{c}\text { Adult } \\
\text { Female }\end{array}$ & $\begin{array}{l}\text { Adult } \\
\text { Male }\end{array}$ & $\begin{array}{c}\text { Adult } \\
\text { Female }\end{array}$ \\
\hline Walking & 35.17 & 29.31 & 2.06 & 1.71 & 1209.60 & 1008.00 & 172.80 & 144.00 \\
\hline Standing & 16.12 & 11.72 & 0.94 & 0.69 & 554.40 & 403.20 & 79.20 & 57.60 \\
\hline Stroller & \multicolumn{2}{|c|}{ Child } & \multicolumn{2}{|c|}{ Child } & \multicolumn{2}{|c|}{ Child } & \multicolumn{2}{|c|}{ Child } \\
\hline Walking & \multirow{2}{*}{\multicolumn{2}{|c|}{122.97}} & \multicolumn{2}{|c|}{0.08} & \multicolumn{2}{|c|}{705.60} & \multicolumn{2}{|c|}{100.80} \\
\hline Standing & & & \multicolumn{2}{|c|}{0.04} & \multicolumn{2}{|c|}{352.80} & \multicolumn{2}{|c|}{50.40} \\
\hline
\end{tabular}

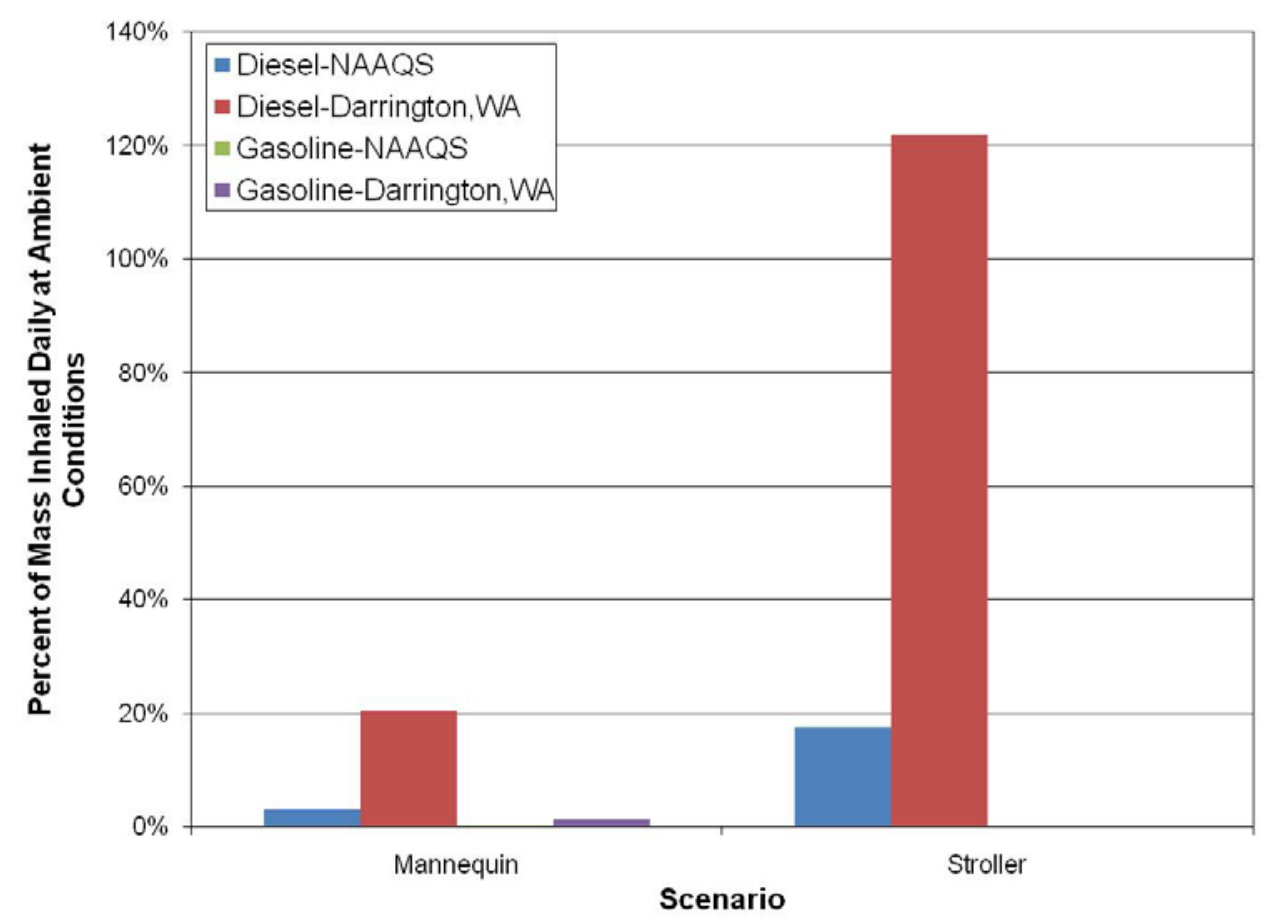

Figure 4-16: Comparison of mass inhaled due to 264 drive-bys and mass inhaled daily.

It can be seen from Table 4-8 and Figure 4-16 that in a relatively dirty city, where the ambient pollution level is equivalent to the National Ambient Air Quality Standard of $35 \mu \mathrm{g} / \mathrm{m}^{3}, 264$ average diesel vehicle drive-by incidents can increase the mass inhaled by an adult and a child by as much as $3 \%$ and 17\%, respectively. Additionally, 264 average gasoline vehicle drive-by incidents can increase the mass inhaled by an adult and a child by as much as $0.17 \%$ and $0.01 \%$, respectively. In a relatively clean city, where the 
ambient pollution level is equivalent to $5 \mu \mathrm{g} / \mathrm{m}^{3}$ which is the ambient PM concentration in Darrington, WA, 264 average diesel drive-by incidents can increase the mass inhaled by an adult and a child by as much as $20 \%$ and $122 \%$, respectively. The same number of drive-by incidents with a gasoline vehicle ranges from $0 \%$ to $2 \%$. These results show that while 264 drive-by incidents with the diesel test vehicle is much more significant than the same number of drive-by incidents with the gasoline vehicle. However, because there are many cities that have ambient PM concentrations that are greater than the NAAQS for PM, the increased mass inhaled daily due to drive-bys with the test vehicles used in this study isn't much more than would be the case in one of those cities. Therefore, the mass inhaled due to 264 drive-by incidents with test vehicles is small compared to typical daily inhalation. Furthermore, more particulate matter was measured for the stroller scenario as opposed to the adult pedestrian scenario during the diesel vehicle drive-bys. However, the opposite was true for the gasoline vehicle drive-bys.

In addition to effects of multiple average drive-by incidents on daily inhalation, the effects of per inhalation exposure were considered. For this analysis, incidents from each scenario with the highest particulate concentrations observed were aligned with the inspiration of a breath. To determine if this inhaled mass was significant, it was compared to the calculated mass of PM inhaled per breath at the same ambient conditions mentioned previously. These values are shown in Table 4-9 where walking and standing correspond to inspiration lengths of 2.5 and 1.5 seconds, respectively. This data can be seen more visually in Figure 4-17.

Table 4-9: Mass inhaled per breath during worst drive-by incident and at different ambient conditions.

\begin{tabular}{|c|c|c|c|c|c|c|c|c|}
\hline Scenario & \multicolumn{2}{|c|}{ Diesel Drive-by } & \multicolumn{2}{|c|}{ Gasoline Drive-by } & \multicolumn{2}{|c|}{ NAAQS } & \multicolumn{2}{|c|}{ Darrington, WA } \\
\hline Mannequin & $\begin{array}{l}\text { Adult } \\
\text { Male }\end{array}$ & $\begin{array}{c}\text { Adult } \\
\text { Female }\end{array}$ & $\begin{array}{l}\text { Adult } \\
\text { Male }\end{array}$ & $\begin{array}{l}\text { Adult } \\
\text { Female }\end{array}$ & $\begin{array}{c}\text { Adult } \\
\text { Male }\end{array}$ & $\begin{array}{c}\text { Adult } \\
\text { Female }\end{array}$ & $\begin{array}{l}\text { Adult } \\
\text { Male }\end{array}$ & $\begin{array}{l}\text { Adult } \\
\text { Female }\end{array}$ \\
\hline Walking & 0.2979 & 0.2480 & 0.0116 & 0.0097 & 0.0350 & 0.0291 & 0.0050 & 0.0042 \\
\hline Standing & 0.1017 & 0.0741 & 0.0039 & 0.0028 & 0.0096 & 0.0070 & 0.0014 & 0.0010 \\
\hline Stroller & \multicolumn{2}{|c|}{ Child } & \multicolumn{2}{|c|}{ Child } & \multicolumn{2}{|c|}{ Child } & \multicolumn{2}{|c|}{ Child } \\
\hline Walking & \multicolumn{2}{|c|}{0.4426} & \multirow{2}{*}{\multicolumn{2}{|c|}{0.0015}} & \multirow{2}{*}{\multicolumn{2}{|c|}{0.0233}} & \multirow{2}{*}{\multicolumn{2}{|c|}{$\frac{0.0033}{0.0009}$}} \\
\hline Standing & \multicolumn{2}{|c|}{0.1534} & & & & & & \\
\hline
\end{tabular}




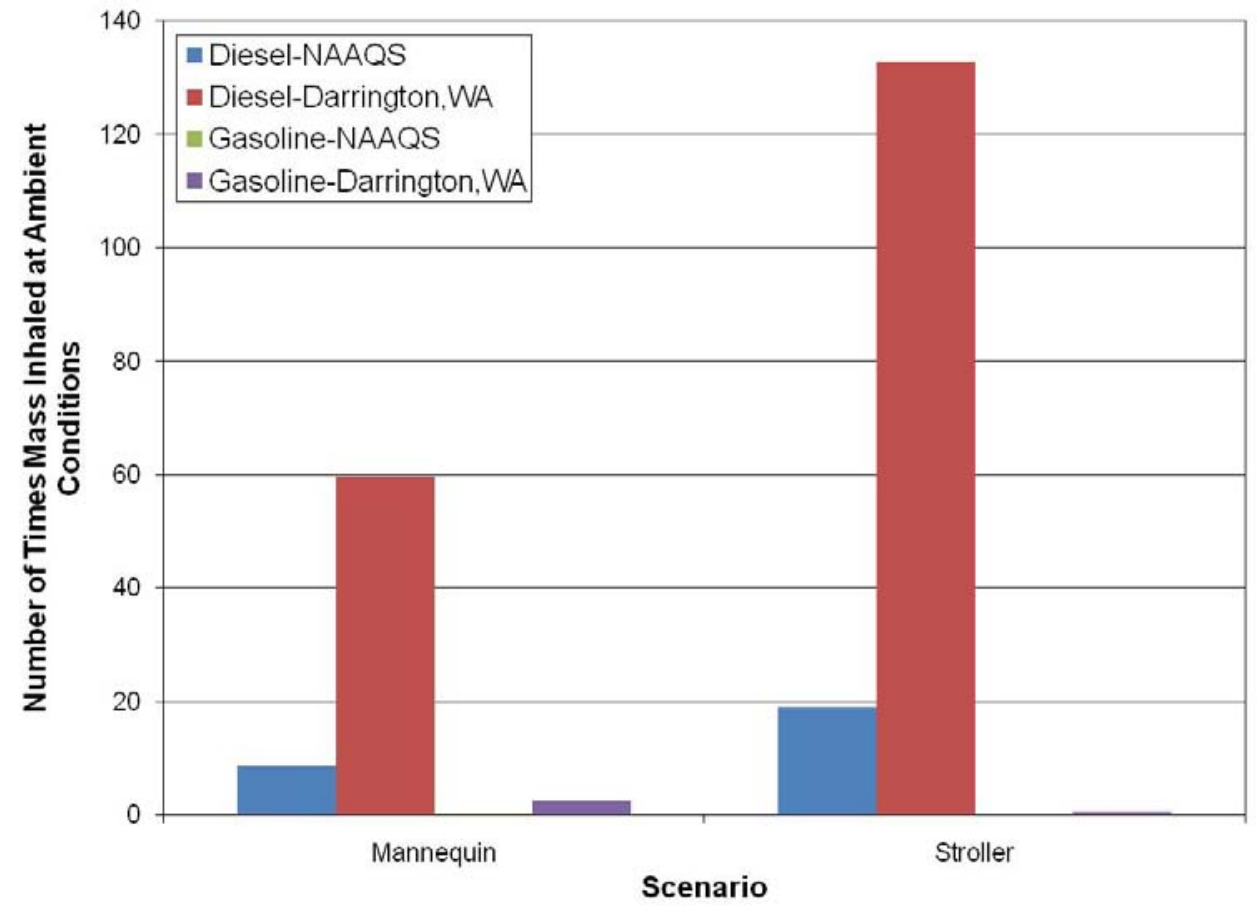

Figure 4-17: Comparison of mass inhaled per breath during drive-by and at ambient.

As shown in Table 4-9 and Figure 4-17, at the same ambient particulate concentrations an adult and a child could inhale as much as 10 and 25 times more particulate matter mass, respectively, in a single inhalation during a diesel vehicle driveby incident from the diesel test vehicle than at ambient conditions. Similarly, a drive-by from the gasoline test vehicle could only increase the mass inhaled per breath by a maximum of 0.5 times. In a clean city, however, the same exposure by the diesel test vehicle can increase mass inhaled by as much as $42 \%$ and $116 \%$ and as much as 82 and 149 times more mass per breath than at ambient conditions. Likewise, in a clean city, the gasoline test vehicle would increase daily mass inhaled by $1.2 \%$ and $0.1 \%$ and as much as 2 times more mass per breath. However, without short-term health effects understanding, it is not possible to project the effect of brief, highly elevated particulate matter levels on a roadside pedestrian. 


\section{Conclusions and Recommendations}

\subsection{Conclusions}

The results from this study indicate that the PM number and mass concentrations near a roadway during a drive-by incident can easily reach number and mass concentrations of several million particles/cc and hundreds of $\mu \mathrm{g} / \mathrm{m}^{3}$, respectively. The highest concentrations observed with the diesel test vehicle were 2.2 million particles/cc and $1400 \mu \mathrm{g} / \mathrm{m}^{3}$, respectively. The average incident mass concentrations can be as high as those used in human dose studies $\left(300 \mu \mathrm{g} / \mathrm{m}^{3}\right)$ while peak concentrations can be up to 4 times higher than those concentrations. Additionally, these average incident concentrations can have durations as long as 25 seconds.

During hard acceleration, the diesel and gasoline test vehicles used in this study had similar particle size distributions. However, the diesel test vehicle emitted at least 6 times as many particles and at least 7 times more PM mass than the gasoline test vehicle. While the diesel test vehicle emitted a higher number of particles, the particles from the gasoline test vehicle were usually smaller (i.e., particle diameter of about $48 \mathrm{~nm}$ for the gasoline vehicle and $86 \mathrm{~nm}$ for the diesel vehicle). Nevertheless, the particles that carried most of the mass in each test vehicle's exhaust were typically about the same size with the peak mass occurring in particles of about $133 \mathrm{~nm}$ in diameter.

The results from this study show that particulate matter concentrations near roadways during drive-by incidents with the diesel test vehicle easily reached or exceeded for brief periods of time those low PM concentrations $\left(300 \mu \mathrm{g} / \mathrm{m}^{3}\right)$ which are capable of causing acute health effects. Because the diesel test vehicle used in this study represents an in-use minimum PM expulsion for medium and heavy duty diesel vehicles, actual in-use vehicles could emit PM concentrations which are an order of magnitude greater than those observed in this study. Particulate matter concentrations due to drivebys with the gasoline test vehicle, on the other hand, did not reach those same concentrations and were typically more than 15 times less than those of the diesel vehicle. This does not, however, imply that all gasoline vehicles will have such low PM emissions. 
PM exposure to children, infants, or people near roadways during drive-by incidents has a direct relationship with the position of the pedestrian with respect to the tailpipe of the passing vehicle as well as the operating condition of the passing vehicle. In this study, the results obtained with the diesel test vehicle showed higher exposure at the height of a stroller during hard acceleration events where as higher concentrations of PM were measured at the height of an adult's mouth during medium acceleration and cruising events. Additionally, the results showed that for some incidents the mannequin case had higher exposure than the without mannequin case, and vice versa. This implies that the results are inconclusive as to whether or not a body increases or decreases exposure. However, external factors such as meteorological conditions could affect all of these results significantly.

PM exposure to children, infants, and people near roadways during drive-by incidents has a direct relationship with the operating conditions of the passing vehicle. It was shown that lower PM concentrations result when the power, load, and engine speed of the passing vehicle are lower. The highest PM concentrations (exposures) are attributed to acceleration of the vehicle with high percent power and load.

For the case of a particularly well-maintained 2006 diesel fueled Dodge Ram 2500 , the mass of particulate matter inhaled during a theoretical maximum number of drive-by incidents (264) by a pedestrian near a roadway is small (3\% to $17 \%)$ compared to the mass inhaled daily at ambient conditions. However, on a per breath basis the mass of particulate matter inhaled is large ( 8.5 to 19 times) compared to the mass inhaled at ambient conditions. For the case of a particularly well-maintained 2001 gasoline fueled Dodge Ram 1500, the mass of particulate matter inhaled due to 264 drive-by incidents is orders of magnitude smaller $(0.01 \%$ to $0.17 \%)$ than the mass inhaled daily at ambient conditions. On a per breath basis, the mass inhaled due to a drive-by with the gasoline test vehicle is equivalent to or less than the mass inhaled at ambient conditions ( 0.5 to 1 times).

\subsection{Recommendations}

Based on the results obtained in this study, it is recommended that future research in this area attempt to map the exhaust plume near a roadway as a vehicle drives past 
under different operating conditions. Being able to determine the dilution ratio is recommended because it can give insight as to the reason for the observed particle size distributions. A larger number of vehicles having different exhaust configurations, utilizing different fuels, and having different model years should be considered as well. It is also recommended that different locations for exposure, such as inside a following vehicle, be considered. Additionally, future research should put more emphasis on the comparison between near tailpipe and near roadway exposure/emissions. Future research should also be more consistent in measuring NOx emissions and exposure in addition to determining a correlation between carbon monoxide and particulate matter. It is also recommended that the flow rate of exhaust through the test vehicles' tailpipes be measured or known. This will help determine a comparison between the results from different vehicles as well as be beneficial in mapping exhaust plumes. 


\section{Bibliography}

1. Geiser, M., Rothen, B., Kapp, N., Schürch, S., Kreyling, W., Schulz, H., Semmler, M., Hof, V., Heyder, J., Gehr, P. "Ultrafine Particles Cross Cellular Membranes by Nonphagocytic Mechanisms in Lungs and in Cultured Cells." Environmental Health Perspectives, Vol. 113, No. 11, 2005.

2. Dawson, S.V., Alexeeff, G.V., Budroe, J.D., Campleman, S., Collins, J.F., Fowles, J.R., Holtzman, D.A., Lam, R.F., Lewis, D.C., Lipsett, M.J., Marty, M.A., Mycroft, F.J., Ostro, B.D., Polakoff, J.S., Vork, K. "Proposed Identification of Diesel Exhaust as a Toxic Air Contaminant." California Environmental Protection Agency Office of Environmental Health Hazard Assessment Air Toxicology and Epidemiology Section. May 1998.

3. Geiser, M., Rothen, B., Kapp, N., Schürch, S., Kreyling, W., Schulz, H., Semmler, M., Hof, V., Heyder, J., Gehr, P. "Ultrafine Particles Cross Cellular Membranes by Nonphagocytic Mechanisms in Lungs and in Cultured Cells." Environmental Health Perspectives, Vol. 113, No. 11, 2005.

4. "Particulate Matter Heath Risk Assessment for Selected Urban Areas." EPA 452/R-05007a, 2005.

5. Roholm, K. "The Fog Disaster in the Meuse Valley, 1930: A Fluorine Intoxication.” The Journal of Industrial Hygiene and Toxicology, Vol. 19, 1937:126-137.

6. Camps, F.E.. “Gradwohl's Legal Medicine, 3rd Edition.” Bristol: John Wright \& Sons Ltd, 1976:236.

7. Hunt, A., Abraham, J.L., Judson, B., Berry, C.L. "Toxicologic and Epidemiologic Clues from the Characterization of the 1952 London Smog Fine Particulate Matter in Archival Autopsy Lung Tissues - Research.” Environmental Health Perspectives, Vol. 111, No. 9, 2003.

8. "Carcinogenic Effects of Exposure to Diesel Exhaust." NIOSH Current Intelligence Bulletin 50. DHHS (NIOSH) Publication No. 88-116. Centers for Disease Control, Atlanta, GA, 1988.

9. "Proposed Identification of Diesel Exhaust as a Toxic Air Contaminant, as Approved by the Scientific Review Panel on April 22, 1998 and Part B: Health Risk Assessment for Diesel Exhaust." CARB/OEHHA, 1998.

10. "Proposed Identification of Diesel Exhaust as a Toxic Air Contaminant: Initial Statement of Reasons for Rulemaking." CARB/OEHHA, 1998.

11. "Diesel Emissions and Lung Cancer: Epidemiology and Quantitative Risk Assessment A Special Report of the Institute's Diesel Epidemiology Expert Panel.” HEI, 1999. 
12. Woodruff, T., Grillo, J., Schoendorf, K. "The Relationship Between Selected Causes of Postneonatal Infant Mortality and Particulate Air Pollution in the United States."

Environmental Health Perspectives, Vol. 105, 1997:608-612.

13. Janssen, N.A.H., Lanki, T., Hoek, G., Vallius, M., Hartog, J.J., Grieken, R., Pekkanen, J., Brunekreef, B. "Associations Between Ambient, Personal, and Indoor Exposure to Fine Particulate Matter Constituents in Dutch and Finnish Panel of Cardiovascular Patients." Occupational and Environmental Medicine, Vol. 62, 2005:868-877.

14. Peters, A., Pope, A.C. "Cardiopulmonary Mortality and Air Pollution." Lancet, Vol. 360, 2002:1184.

15. Hong, Y., Lee, J., Kim, D.H., Ha, E., Schwartz, J., Christiani, D.C. "Effects of Air Pollutants on Acute Stroke Mortality." Environmental Health Perspectives, Vol. 110, No. $2,2002$.

16. Kilburn, K.H. "Effects of Diesel Exhaust on Neurobehavioral and Pulmonary Functions." Environmental Health, Vol. 55, No. 1, 2000:11-17.

17. Yin, X.J., Shafer, R., Ma J.Y.C., Antonini, J.M., Weissman, D.D., Siegel, P.D., Barger, M.W., Roberts, J.R., and Ma, K.H. "Alteration of Pulmonary Immunity to Listeriamonocytogenes by Diesel Exhaust Particles (DEPS). I. Effects of DEPS on Early Pulmonary Responses.” Environmental Health Perspectives, Vol. 110, No. 11, 2002.

18. Rudell, B., Ledin, M.C., Hammarstrom, U., Stjernberg, N., Lundback, B., Sandstrom, T. "Effects on Symptoms and Lung Function in Humans Experimentally Exposed to Diesel Exhaust." Occupational and Environmental Medicine, Vol. 53, 1996:658-662.

19. Lloyd, A.C., Cackette, T.A. "Diesel Engines: Environmental Impact and Control." Journal of Air Waste Management, Vol. 51, 2001:809-847.

20. Cruts, B., van Etten, L., Tornqvist, H., Blomberg, A., Sandstrom, T., Mills, N.L., Borm, P.J. "Exposure to Diesel Exhaust Induces Changes in EEG in Human Volunteers." Particle and Fibre Toxicology, Vol. 5, 2008:4.

21. Holgate, S.T. "Health Effects of Acute Exposure to Air Pollution." Research Report 112. Health Effects Institute, Boston MA, 2003.

22. Mills, N.L., Tornqvist, H., Robinson, S.D., Gonzalez, M., Darnley, K., MacNee, W., Boon, N.A., Donaldson, K., Blomberg, A., Sandstrom, T., Newby, D.E. "Diesel Exhaust Inhalation Causes Vascular Dysfunction and Impaired Endogenous Fibrinolysis." Circulation, Vol. 112, 2005:3930-3936.

23. Mills, N.L., Tornqvist, H., Gonzalez, M.C., Vink, E., Robinson, S.D., Soderberg, S., Boon, N.A., Donaldson, K., Sandstrom, T., Blomberg, A., Newby, D.E. "Ischemic and Thrombotic Effects of Dilute Diesel-Exhaust Inhalation in Men with Coronary Heart Disease." The New England Journal of Medicine, Vol. 357, 2007:1075-1082. 
24. Peretz, A., Kaufman, J.D., Trenga, C.A., Allen, J., Carlsten, C., Aulet, M.R., Adar, S.D., Sullivan, J.H. "Effects of Diesel Exhaust Inhalation on Heart Rate Variability in Human Volunteers." Environmental Research, Vol. 107, 2008:178-184.

25. Peretz, A., Peck, E.C., Bammler, T.K., Beyer, R.P., Sullivan, J.H., Trenga, C.A., Srinouanprachnah, S., Farin, F.M., Kaufman, J.D. "Diesel Exhaust Inhalation and Assessment of Peripheral Blood Mononuclear Cell Gene Transcription Effects: An Exploratory Study of Healthy Human Volunteers." Inhalation Toxicology, Vol. 19, 2007:1107-1119.

26. Peretz, A., Sullivan, J.H., Leotta, D.F., Trenga, C.A., Sands, F.N., Allen, J., Carlsten, C., Wilkinson, C.W., Gill, E.A., Kaufman, J.D. "Diesel Exhaust Inhalation Elicits Acute Vasoconstriction in Vivo." Environmental Health Perspectives, Vol. 116, 2008:937-942.

27. Peters, A., von Klot, S., Heier, M., Trentinaglia, I., Hormann, A., Wichmann, H.E., Lowel, H. "Exposure to Traffic and the Onset of Myocardial Infarction." The New England Journal of Medicine, Vol. 351, 2004:1721-1730.

28. Salvi, S., Blomberg, A., Rudell, B., Kelly, F., Sandstrom, T., Holgate, S.T., Frew, A. "Acute Inflammatory Responses in the Airways and Peripheral Blood After Short-term Exposure to Diesel Exhaust in Healthy Human Volunteers." American Journal of Respiratory and Critical Care Medicine, Vol. 159, 1999:702-709.

29. Tornqvist, H., Mills, N.L., Gonzalez, M., Miller, M.R., Robinson, S.D., Megson, I.L., Macnee, W., Donaldson, K., Soderberg, S., Newby, D.E., Sandstrom, T., Blomberg, A. "Persistent Endothelial Dysfunction in Humans After Diesel Exhaust Inhalation." American Journal of Respiratory and Critical Care Medicine, Vol. 176, 2007:395-400.

30. US EPA. Air Quality Criteria for Particulate Matter. US Environmental Protection Agency, Washington, DC, EPA 600/P-99/002aF-bF, 2004.

31. "National Ambient Air Quality Standards (NAAQS)." $<\mathrm{http} / / /$ www.epa.gov/air/criteria.html\#5>.

32. “Occupational Health Regulations.” $<$ http://www.dieselnet.com/standards/us/ohs.html $>$.

33. Tamura, T.M., Eisinger, D.S., Hafner, H.R., Chinkin, L.R., Roberts, P.T., Black, K.N., Clark, N.N., McMurry, P.H., Winer, A.M. "Transportation and Particulate Matter: Assessment of Recent Literature and Ongoing Research.” Report STI-904751-2669-FR. FHWA: Washington, DC, 2005.

34. McCarthy, M.C., Eisinger, D.S., Hafner, H.R., Chinkin, L.R., Roberts, P.T., Black, K.N., Clark, N.N., McMurry, P.H., Winer, A.M. "Particulate Matter: A Strategic Vision for Transportation-Related Research.” Environmental Science and Technology Online, Vol. 40, No. 18, 2007:5593-5599.

$<$ http://pubs.acs.org/subscribe/journals/esthag/40/118/html/091506feature_eisinger.html>.

35. "Ambient Air Monitoring Strategy for State, Local, and Tribal Air Agencies." Office of Air Quality Planning and Standards, Research Triangle Park, NC, 2008. 
36. Clark, N.N., Gautam, M., Boyce, J., Xie, W., Mehta, S., Jarrett, R., Rapp, B. "HeavyDuty Vehicle Exhaust Plume Study in the NASA/Langley Wind Tunnel." SAE/JSAE Spring Fuels \& Lubricants Meeting, Yokohama, Japan 2003. JSAE Paper 20030290.

37. Gautam, M., Clark, N.N., Mehta, S., Boyce, J. "Concentrations and Size Distributions of Particulate Matter Emissions from a Class-8 Heavy-duty Diesel Truck Tested in a Wind Tunnel." SAE/JSAE Spring Fuels \& Lubricants Meeting, Yokohama, Japan 2003, JSAE Paper 20030291.

38. Kittelson, D.B., W.F. Watts, J. Johnson. "Diesel Aerosol Sampling Methodology - CRC E-43: Final Report." University of Minnesota, Report for the Coordinating Research Council, 19 August 2002. <http://www.crcao.com/reports/recentstudies0002/UMN\%20Final\%20E-43\%20Report.pdf>.

39. Brown, J.E. "Development and Application of On-Road Measurement Capabilities." National Risk Management Research Laboratory, Research Triangle Park, NC 2001.

40. Kittleson, D.B., Johnson, J., Watts, W.F., Wei, Q., Drayton, M., Paulsen, D., Bukowiecki, N. "Diesel Aerosol Sampling in the Atmosphere." Government/Industry Meeting, Washington D.C. 2000. SAE 2000-01-2212.

41. Maricq, M.M., Chase, R.E., Podsiadlik, D.H., Vogt, R. "Vehicle Exhaust Particle Size Distributions: A Comparison of Tailpipe and Dilution Tunnel Measurements." International Spring Fuels and Lubricants Meeting, Dearborn, MI 1999. SAE 1999-011461.

42. Majewski, W.A. "Diesel Particulate Matter." $<$ http://www.dieselnet.com/tech/dpm.html $>$.

43. Khair, M.K., Jääskeläinen, H. "Emission Formation in Diesel Engines." $<\mathrm{http}: / /$ dieselnet.com/tech/diesel_emiform.html\#pm>.

44. Hall, D.E., Stradling, R.J., Rickeard, D.J., Martini, G., Morato-Meco, A., Hagemann, R., Szendefi, J., Rantanen, L., Zemroch, P.J., Heinze, P., Thompson, N. "Measurement of the number and mass weighted size distributions of exhaust particles emitted from european heavy duty engines." CONCAWE Report 01/51, January 2001.

45. Parkin, C. "Update on the UN-ECE Particle Measurement Programme (PMP)." UK Department of Transport. <http://wwwdiva.eng.cam.ac.uk/energy/nickparticle/particle_meeting_08/CParkin(DfT)_PMP.pdf>.

46. Bagley, S.T., Baumgard, K.J., Gratz, L.D., Johnson, J.J., Leddy, D.G. "Characterization of Fuel and After-Treatment Device Effects on Diesel Emissions." Health Effects Institute, Cambridge, MA, Report 76, 1996. <http://healtheffects.org/Pubs/st76.htm>.

47. Baumgard, K.J., Johnson, J.H. "The Effect of Fuel and Engine Design on Diesel Exhaust Particle Size Distribution.” SAE Technical Paper 960131.

48. Mayer, A. "VERT: Diesel Nano-Particulate Emissions: Properties and Reduction Strategies." SAE Technical Paper 980539. 
49. Kittelson, D.B. "Characterization of Diesel Particles in the Atmosphere." AP-2 Project Group Final Report, Coordinating Research Council, Alpharetta, GA, 1998.

50. Wedekind, B., Andersson, J., Hall, D., Stradling, R., Barnes, C., Wilson, G. "DETR/SMMT/CONCAWE Particle Research Program: Heavy-Duty Results." SAE Technical Paper 2000-01-2851.

51. Andersson, J.D., Wedekind, B. "DETR/SMMT/CONCAWE Particulate Research Programme.” Ricardo Consulting Engineers, Summary Report DP01/0515, May 2001. $<$ http://www.ricardo.com/downloads/SummaryReport.pdf $>$.

52. ACEA. "ACEA programme on emissions of fine particles from passenger cars." European Automobile Manufacturers Association, Brussels, Belgium, July 2002, $<$ http://www.acea.be/ACEA/20020702PublicationPMReport.pdf $>$.

53. Dreher, W., Harscher, A., Nisch, W., Burkhardt, C. "TEM analysis of diesel nanoparticle emission to get a geometric correlation to SMPS mobility diameter." Proc. of the 6th ETH Conference on Nanoparticle Measurement, Zurich, August 2002.

54. Abdul-Khalek, I.S. "Diesel Exhaust Particle Size: Measurement Issues and Trends." SAE Technical Paper 980525.

55. Merkel, G.A., Cutler, W.A., Warren, C.J. "Thermal Durability of Wall-Flow Ceramic Diesel Particulate Filters.” SAE Technical Paper 2001-01-0190.

56. Abbass, M.K., Andrews, G.E., Ishaq, R.B., Williams, P.T., Bartle, K.D. “A Comparison of the Particulate Composition Between Turbocharged and Naturally Aspirated DI Diesel Engines." SAE Technical Paper 910733.

57. Wachter W.F. "Analysis of Transient Emission Data of a Model Year 1991 Heavy Duty Diesel Engine." SAE Technical Paper 900443.

58. EPA. "Exposure and Human Health Reassessment of 2,3,7,8-Tetrachlorodibenzo-pDioxin (TCDD) and Related Compounds." Draft Document, US Environmental Protection Agency, September 2000.

$<$ http://www.epa.gov/ncea/pdfs/dioxin/part1 and2.htm>.

59. "Six Common Air Pollutants." <http://www.epa.gov/air/urbanair/>.

60. Code of Federal Regulations, Title 40, Part 86. "Protection of Environment - Control of Emissions from New and In-Use Highway Vehicles and Engines." US Government Printing Office, Washington DC, 2006.

61. Code of Federal Regulations, Title 40, Part 50. "Protection of Environment - National Primary and Secondary Ambient Air Quality Standards." US Government Printing Office, Washington DC, 2006. 
62. "Diesel Exhaust: a Critical Analysis of Emissions, Exposure and Health Effects. A Special Report of Institute's Diesel Working Group.” Health Effects Institute, Cambridge, MA, 1995.

63. "Diesel Fuel and Exhaust Emissions - Environmental Health Criteria 171." World Health Organization, Geneva, Switzerland, 1996.

64. Reger, R., Hancock, J., Hankinson, J., Hearl, F., Merchant, J. “Coal Miners Exposed to Diesel Exhaust Emissions.” Annals of Occupational Hygiene, Vol. 26, 1982:799-815.

65. Gamble, J., Jones, W., Minshall, S. "Epidemiological-Environmental Study of Diesel Bus Garage Workers: Chronic Effects of Diesel Exhaust on the Respiratory System." Environmental Research, Vol. 44, 1987:6-17.

66. Heinrich, U., Peters, L., Funcke, W., Pott, F., Mohr, U., Stöber, W. "Investigation of Toxic and Carcinogenic Effects of Diesel Exhaust in Long-Term Inhalation Exposure of Rodents." Developments in Toxicology and Environmental Science, Vol. 10, 1982:225242.

67. Heinrich, U., Muhle, H., Takenaka, S., Ernst, H., Fuhst, R., Mohr, U., Pott, F., Stöber, W. "Chronic Effects on the Respiratory Tract of Hamsters, Mice and Rats After LongTerm Inhalation of High Concentrations of Filtered and Unfiltered Diesel Engine Emissions." Journal of Applied Toxicology, Vol. 6, 1986:383-395.

68. Heinrich, U., Fuhst, R., Rittinghausen, S., Creutzenberg, O., Bellmann, B., Koch, W., Levsen, K. "Chronic Inhalation Exposure of Wistar Rats and Two Different Strains of Mice to Diesel Engine Exhaust, Carbon Black, and Titanium Dioxide." Inhalation Toxicology, Vol. 7, 1995:533-556.

69. Brightwell, J., Fouillet, X., Cassano-Zoppi, A.L., Gatz, R., Duchosal, F. "Neoplastic and Functional Changes in Rodents After Chronic Inhalation of Engine Exhaust Emissions." Developments in Toxicology and Environmental Science, Vol. 13, 1986:471-485.

70. Ishinishi, N., Kuwabara, N., Nagase, S., Suzuki, T., Ishiwata, S., Kohno, T. "Long-Term Inhalation Studies of Diesel Exhaust on F344 SPF Rats." Developments in Toxicology and Environmental Science, Vol. 13, 1986:349-360.

71. Rudell, B., Sandstrom, T., Stjernberg, N., Kolmodin-Hedman, B. "Controlled Diesel Exhaust Exposure in an Exposure Chamber: Pulmonary Effects Investigated With Bronchoalveolar Lavage.” Journal of Aerosol Science, Vol. 21, Supplement 1, 1990:411414.

72. Rudell, B., Sandström, T., Hammarström, U., Ledin, M.L., Hörstedt, P., Stjernberg, N. "Evaluation of an Exposure Setup for Studying Effects of Diesel Exhaust in Humans." International Archives of Occupational and Environmental Health, Vol. 66, 1994:77-83.

73. Rudell, B., Ledin, M.L., Hammarström, U., Stjernberg, N., Sandström, T. "Effects on Symptoms and Lung Function in Humans Experimentally Exposed to Diesel Exhaust." Occupational Environmental Medicine, Vol. 53, 1996:658-662. 
74. Kahn, G., Orris, P., Weeks, J. “Acute Overexposure to Diesel Exhaust: Report of 13 Cases.” American Journal of Industrial Medicine, Vol. 13, 1988:405-406.

75. Wade III, J.F., Newman, L.S. "Diesel Asthma: Reactive Airways Disease Following Overexposure to Locomotive Exhaust.” Journal of Occupational Medicine, Vol. 35, 1993:149-154.

76. “Air Quality Guidelines - Second Edition.” WHO Regional Office for Europe, Copenhagen, Denmark, 2000.

77. Schwartz, J., Dockery, D.W., Neas, L.M. "Is Daily Mortality Associated Specifically With Fine Particles?” Journal of Air Waste and Management, Vol. 46, No. 10, 1996:927939.

78. Schwartz, J., Slater, D., Larson, T.V., Pierson, W.E., Koenig, J.Q. "Particulate Air Pollution and Hospital Emergency Room Visits for Asthma in Seattle." American Review of Respiratory Disease, Vol. 147, 1993:826-831.

79. Murphy, S.A.M., Berube, K.A., Richards, R. J. "Bioreactivity of Carbon Black and Diesel Exhaust Particles to Primary Clara and Type Ii Epithelial Cell Cultures." Occupational and Environmental Medicine, Vol. 56, No. 12, 1999:813-819

80. Wichmann, H.E., Peters, A. "Epidemiological Evidence of the Effects of Ultrafine Particle Exposure." Philosophical Transactions: Mathematical, Physical and Engineering Sciences, Ultrafine Particles in the Atmosphere, Vol. 358, No. 1775, 2000:2751-2768.

81. Renwick, L.C., Donaldson, K., Clouter, A. "Impairment of Alveolar Macrophage Phagocytosis by Ultrafine Particles." Toxicology and Applied Pharmacology, Vol. 172, No. 2, 2001:119-127.

82. Oberdorster, G., Sharp, Z. "Extrapulmonary Translocation of Ultrafine Carbon Particles Following Whole-Body Inhalation Exposure of Rats." Journal of Toxicology and Environmental Health, Part A, Vol. 65, No. 20, 2002:1531-1543.

83. Chen, F., Phousongphouang, P., Baldauf, R.W., Wiener, R.W., Dimmick, F., Wheeler, M. "Particulate Matter Concentrations Measured in a Residential Neighborhood in Brooklyn, New York City During the Traffic-Related Exposure Study (T-Rex)." Presented at AAAR 2006, St Paul, MN, September 11-15, 2006.

84. Williams, R., Rea, A., Vette, A., Croghan C., Whitaker, D., Wilson, H., Stevens, C., McDow, S., Burke, J., Fortmann, R., Sheldon, L., Thornburg, J., Phillips, M., Lawless, P., Rodes, C., Daughtrey, H. "The design and field implementation of the Detroit Exposure and Aerosol Research Study (DEARS)." Journal of Exposure Science and Environmental Epidemiology 2008. Published online at $<\mathrm{http}: / /$ www.nature.com/jes/journal/vaop/ncurrent/abs/jes200861a.html $>$.

85. Zhu, Y., Hinds, W.C., Kim, S. "Study of ultrafine particles near a major highway with heavy-duty diesel traffic." Atmospheric Environment, Vol. 36, 2002:4323-4335. 
86. Zhu, Y., Hinds, W.C., Kim, S., Sioutas, C. "Concentration and size distribution of ultrafine particles near a major highway." Journal of Air and Waste Management Association, Vol. 52, 2002:1032-1042.

87. Kittelson, D.B., Watts, W.F., Johnson, J.P. "Nanoparticle emissions on Minnesota highways.” Atmospheric Environment, Vol. 38, 2004:9-19.

88. Reponen, T., Grinshpun, S.A., Trakumas, S. "Concentration gradient patterns of aerosol particles near interstate highways in the Greater Cincinnati airshed." Journal of Environmental Monitoring, Vol. 5, 2003:557-562.

89. Westerdahl, D., Fruin, S., Sax, T., Fine, P.M., Sioutas, C. "Mobile platform measurements of ultrafine particles and associated pollutant concentrations on freeways and residential streets in Los Angeles.” Atmospheric Environment, Vol. 39, 2005:35973610 .

90. Shah, S.D., Cocker III, D.R., Miller, J.W., Norbeck, J.M. "Emission Rates of Particulate Matter and Elemental and Organic Carbon from In-Use Diesel Engines." Environmental Science Technology, Vol. 38, No. 9, 2004:2544-2550.

91. Sharma, M., Agarwal, A.K., Bharathi, K.V.L. "Characterization of Exhaust Particulates from Diesel Engine.” Atmospheric Environment, Vol. 39, No. 17, 2005:3023-3028.

92. Wang, J., Storey, J., Domingo, N., Huff, S., Thomas, J., West, B. "Studies of Diesel Engine Particle Emissions During Transient Operations Using an Engine Exhaust Particle Sizer." Aerosol Science and Technology, Vol. 40, No. 11, 2006:1002-1015.

93. Riediker, M., Williams, R., Devlin, R. "Exposure to particulate matter, volatile organic compounds, and other air pollutants inside patrol cars." Environmental Science and Technology, Vol. 37, 2003:2084-2093.

94. Rodes, C., Sheldon, L., Whitaker, D. "Measuring concentrations of selected air pollutants inside California vehicles." Final report to California Air Resources Board, 1998. Contract No. 95-339.

95. Fitz, D. R., Winer, A. M., Colome, S. "Characterizing the Range of Children's Pollutant Exposure During School Bus Commutes." Final Report Prepared for the California Resources Board, 2003. This document is available in Docket EPA-HQ-OAR-2005-0036.

96. Sabin, L.D., Behrentz, E., Winer, A.M. "Characterizing the range of children's air pollutant exposure during school bus commutes." Journal of Exposure Analysis and Environmental Epidemiology, Vol. 15, 2005:377-387.

97. Behrentz, E., Sabin, L.D., Winer, A.M. "Relative importance of school bus-related microenvironments to children's pollutant exposure." Journal of Air and Waste Management Association, Vol. 55, 2005:1418-1430.

98. Fruin, S.A., Winer, A.M., Rodes, C.E. "Black carbon concentrations in California vehicles and estimation of in-vehicle diesel exhaust particulate matter exposures." Atmospheric Environment, Vol. 38, 2004:4123-4133. 
99. Adams, H.S., Nieuwenhuijsen, M.J., Colvile, R.N. "Determinants of fine particle (PM2.5) personal exposure levels in transport microenvironments, London, UK." Atmospheric Environment, Vol. 35, 2001:4557-4566.

100. Leung, P.-L., Harrison, R.M. "Roadside and in-vehicle concentrations of monoaromatic hydrocarbons." Atmospheric Environment, Vol. 33, 1999:191-204.

101. Weinhold, B. "Pollutants lurk inside vehicles." Environmental Health Perspectives 2001, 109:A422-A427.

102. Van Wijnen, J.H., Verhoeff, A.P., Jans, H.W.A., Van Bruggen, M. "The exposure of cyclists, car drivers and pedestrians to traffic-related air pollutants." International Archives of Occupational and Environmental Health, Vol. 67, 1995:187-193.

103. Gulliver, J., Briggs, D.J. "Personal exposure to particulate air pollution in transport microenvironments." Atmospheric Environment, Vol. 38, 2004:1-8.

104. Tsai P.-J., Shih T.-S., Chen H.-L., Lee W.-J., Lai C.-H., Liou S.-H. “Assessing and predicting the exposures of polycyclic aromatic hydrocarbons (PAHs) and their carcinogenic potencies from vehicle engine exhausts to highway toll station workers." Atmospheric Environment, Vol. 38, 2004:333-343.

105. Shih, T.-S., Lai, C.-H., Hung, H.-F., Ku, S.-Y., Tsai, P.-J., Yang, T., Liou, S.-H., Loh, C.H., Jaakkola, J.J.K. "Elemental and organic carbon exposure in highway tollbooths: A study of Taiwanese toll station workers." Science of the Total Environment, Vol. 402, 2008:163-170.

106. Sapkota, A., Williams, D., Buckley, T.J. “Tollbooth Workers and Mobile Source-Related Hazardous Air Pollutants: How Protective Is the Indoor Environment?" Environmental Science and Technology, Vol. 59, 2005:2936-2943.

107. Whitby, K.T., Clark, W.E., Marple, V.A., Sverdrup, G.M., Sem, G.J., Willeke, K., Liu, B.Y.H., Pui, D.Y.H. "Characterization of California Aerosols - 1. Size Distributions of Freeway Aerosol.” Atmospheric Environment, Vol. 9, 1975:463-482.

108. Stedman, D.H., Bishop, G.A. "An Analysis of On-Road Remote Sensing As A Tool For Automobile Emissions Control." Final Report to the Illinois Department of Energy and Natural Resources, ILENR/RE-AQ-90/05, 1990.

109. Stedman, D.H., Bishop, G.A., Guenther, P.L., Peterson, J.E., Beaton, S.P., McVey, I.F. "Remote Sensing of On-Road Vehicle Emissions." Final Report to Coordinating Research Council under Contract No. VE-8-1, 1992.

110. Butlet, J.W., Gierczak, C.A., Jesion, G., Stedman, D.H., Lesko, J.M. “On-Road NO Emissions Intercomparison of On-Board Measurements and Remote Sensing." Final Report (Ford Motor Co.), CRC Report No. VE-11-6, Feb., 1994. 
111. Moris, J.A., Bishop, G.A., Stedman, D.H. “On-Road Remote Sensing of Heavy-duty Diesel Truck Emissions in the Austin-San Marcos Area: August 1998." Report prepared for Espey, Huston \& Assoc., Inc., Nov., 1998.

112. Slott, R.S. "E-23 Remote Sensing in Four Cities to Determine the Change in On-Road Vehicle Fleet Emissions Over Time." Prepared for the Coordinating Research Council. Contract No. E-23.

113. Kittelson, D. B., Kadue, P.A., Scherrer, H.C., and Lovrien, R.E. "Characterization of Diesel Particles in the Atmosphere.” AP-2 Project Group Final Report, Coordinating Research Council, 1988.

114. Elder, A., J.P. Couderc, R. Gelein, S. Eberly, C. Cox, X. Xia, W. Zareba, P. Hopke, W. Watts, D. Kittelson, M. Frampton, M. Utell, and G. Oberdörster. "Effects of On-Road Highway Aerosol Exposures on Autonomic Responses in Aged, Spontaneously Hypertensive Rats.” Inhalation Toxicology, Vol. 19, 2007:1-12.

115. Kittelson, D. B., W. F. Watts, J. P. Johnson, M. L. Remerowki, E. E. Ische, G. Oberdörster, R. M. Gelein, A. C. Elder, and P. K. Hopke. "On-Road Exposure to Highway Aerosols: 1. Aerosol and Gas Measurements." Inhalation Toxicology, Vol. 16, 2004:31-39.

116. Elder, A., R. Gelein, J. Finkelstein, R. Phipps, M. Frampton, M. Utell, D. Kittelson, W. Watts, P. Hopke, C-H. Jeong, E. Kim, W. Liu, W. Zhao, L. Zhuo, R. Vincent, P. Kumarathasan, and G. Oberdörster. "On-Road Exposure to Highway Aerosols: 2. Exposures of Aged, Compromised Rats," Inhalation Toxicology, Vol. 16, 2004:41-53.

117. Kittelson, D. B., W. F. Watts, and J. P. Johnson. "On-road and Laboratory Evaluation of Combustion Aerosols Part 1: Summary of Diesel Engine Results." Journal of Aerosol Science, Vol. 37, 2006:913-930.

118. Kittelson, D. B., W. F. Watts, J. P. Johnson, J. Schauer, and D. R. Lawson. "On-road and Laboratory Evaluation of Combustion Aerosols Part 2: Summary of Spark Ignition Engine Results.” Journal of Aerosol Science, Vol. 37, 2006:931-949.

119. "National Ambient Air Quality Objectives for Particulate Matter. Part 1: Science Assessment Document." CEPA/FPAC Working Group on Air Quality Objectives and Guidelines.

120. Khalek, I.A. “2007 Diesel Particulate Measurement Research. Final Report Project E-66Phase 3.” Southwest Research Institute, San Antonio, Texas, 2007.

121. Symunds, J.P.R., Reavell, K.S.J., Olfert, J.S., Campbell, B.W., Swift, S.J. "Diesel Soot Mass Calculation in Real-Time with a Differential Mobility Spectrometer." Journal of Aerosol Science, Vol. 38, 2007:52-68.

122. Messer, J.T., Clark, N.N. "Measurement Delays and Modal Analysis for a Transportable Heavy Duty Vehicle Emissions Testing Laboratory." SAE Congress, Detroit 1995, SAE Paper 950218. 
123. Burgess, W.A., Diberardinis, L., Speizer, F.E. "Health Effects of Exposure to Automobile Exhaust -V. Exposure of Toll Booth Operators to Automobile Exhaust." American Industrial Hygiene Association Journal, Vol. 38, No. 4, 1977:184-191.

124. Stedman, D.H., Bishop, G.A., Guenther, P.L., Peterson, J.E., Beaton, S.P., McVey, I.F. "Remote Sensing of On-road Vehicle Emissions." Prepared by the University of Denver for the Coordinating Research Council, Contract No. VE-8-1, 1992.

125. Tsang, H., Kwok, R., Miguel, A.H. "Pedestrian Exposure to Ultrafine Particles in Hong Kong Under Heavy Traffic Conditions.” Aerosol and Air Quality Research, Vol. 8, No. 1, 2008:19-27.

126. Watt, M., Godden, D., Cherrie, J., Seaton, A. "Individual Exposure to Particulate Air Pollution and Its Relevance to Thresholds for Health Effects: A Study of Traffic Wardens." Occupational and Environmental Medicine, Vol. 52, 1995:790-792.

127. Kittleson, D.B., Watts, W.F., Arnold, M.J. "Review of Particulate Matter Sampling Methods." University of Minnesota, 1999.

128. Jarrett, R.P., Clark, N.N. "Evaluation of Methods for Determining Continuous Particulate Matter from Transient Testing of Heavy Duty Diesel Engines." SAE Technical Paper 2001-013-575, 2001.

129. Ajtay, D., Weilenmann, M. "Compensation of the Exhaust Gas Transport Dynamics for Accurate Instantaneous Emission Measurements." Environmental Science and Technology, Vol. 38, No. 19, 2004:5141-5148.

130. Madireddy, R.M., Clark, N.N. "Sequential Inversion Technique and Differential Coefficients Approach for Accurate Instantaneous Measurement." International Journal of Engine Research, Vol. 7, No. 6, 2006:437-446.

131. McLean, J.R. “Operations: Theory and Practice.” Published by Taylor \& Francis US, 1989. p. 11.

132. Kittleson, D.B., Watts, W.F., Johnson, J.P., Zarling, D., Schauer, J.J., Kasper, A., Baltensperger, U., Burtscher, H. "Gasoline Vehicle Exhaust Particle Sampling Study." Proceedings of the US Department of Energy $9{ }^{\text {th }}$ Diesel Engine Emissions Reduction Conference (DEER 2003), Newport, RI, August 2003.

133. Zielinska, B., Sagebiel, J., McDonald, J.D., Whitney, K., Lawson, D.R. "Emissions Rates and Comparative Chemical Composition from Selected In-use Diesel and Gasoline Fueled Vehicles." Journal of Air and Waste Management Association, Vol. 54, 2004:1138-1150.

134. Jackson, T.R. "Fleet Characterization Data for MOBILE6: Development and Use of Age Distributions, Average Annual Mileage Accumulation Rates, and Projected Vehicle Counts for Use in MOBILE6." United States Environmental Protection Agency. September 2001. 
135. Clark, N.N., Gautam, M., Wayne, S.W., Lyons, D.W., Thompson, G. "Heavy Duty Vehicle Chassis Inventory, Air Quality Modeling, Source Apportionment and Air Toxics Emissions Inventory. Project E-55/59: Phase 2 Final Report.” Submitted to Coordinating Research Council by West Virginia University Research Corporation, Morgantown, WV, July 2005.

136. Adams, W.C. "Measurement of Breathing Rate and Volume in Routinely Performed Daily Activity." Final Report Contract No. A033-205. Prepared for the California Air Resources Board Research Division, Sacramento, CA. Submitted by Human Performance Laboratory, Davis, CA. 


\section{Appendix A: Gaseous analyzer regression equations}

The gaseous analyzers were calibrated using polynomial regression with 11 points. The $\mathrm{CO}$ analyzer was calibrated over a range from 0 to $99.8 \mathrm{ppm}$. The $\mathrm{CO}_{2}$ analyzer was calibrated over a range from 0 to $2.003 \%$. The NOx analyzer was calibrated over a range from 0 to $101 \mathrm{ppm}$. Both the measured concentrations and their corresponding analog voltage outputs were recorded. As shown, in Figure 7-1, Figure $7-2$, and Figure $7-3,3^{\text {rd }}$ order polynomial regression equations were developed for the $\mathrm{CO}$ and $\mathrm{CO}_{2}$ calibrations and a $2^{\text {nd }}$ order polynomial regression equation was developed from the NOx calibrations. These equations were incorporated into the Visual Basic for Applications data reduction program written by the author in order to predict concentrations from the recorded voltages.

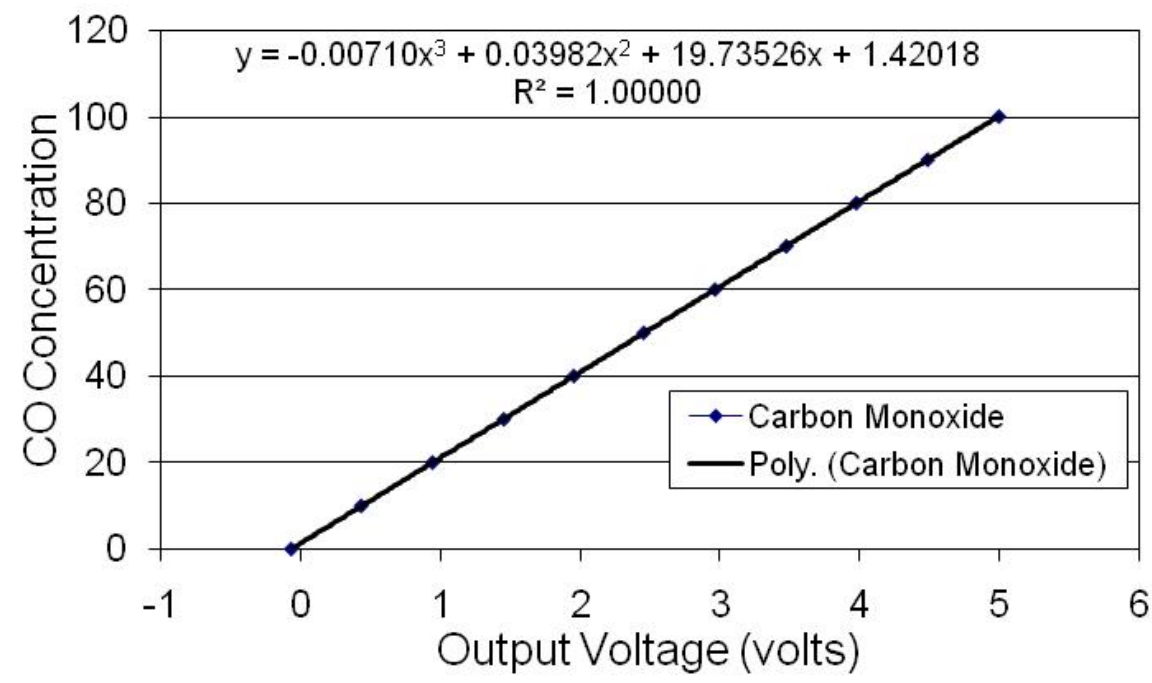

Figure 7-1: Calibration polynomial regression equation for $\mathrm{CO}$ analyzer. 


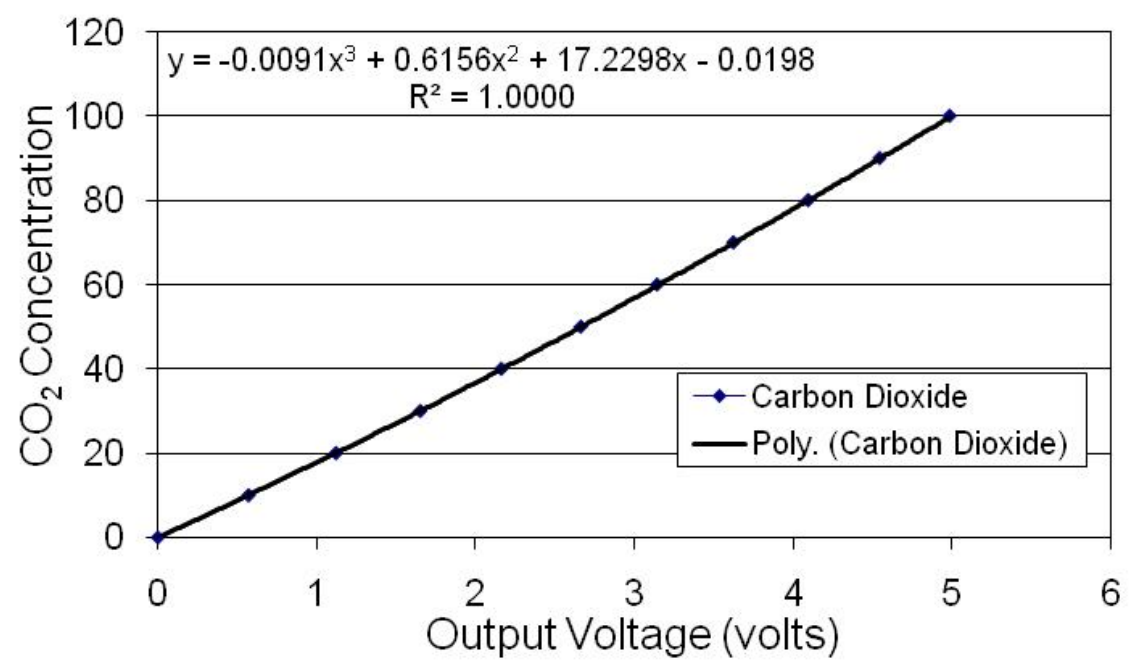

Figure 7-2: Calibration polynomial regression equation for $\mathrm{CO}_{2}$ analyzer.

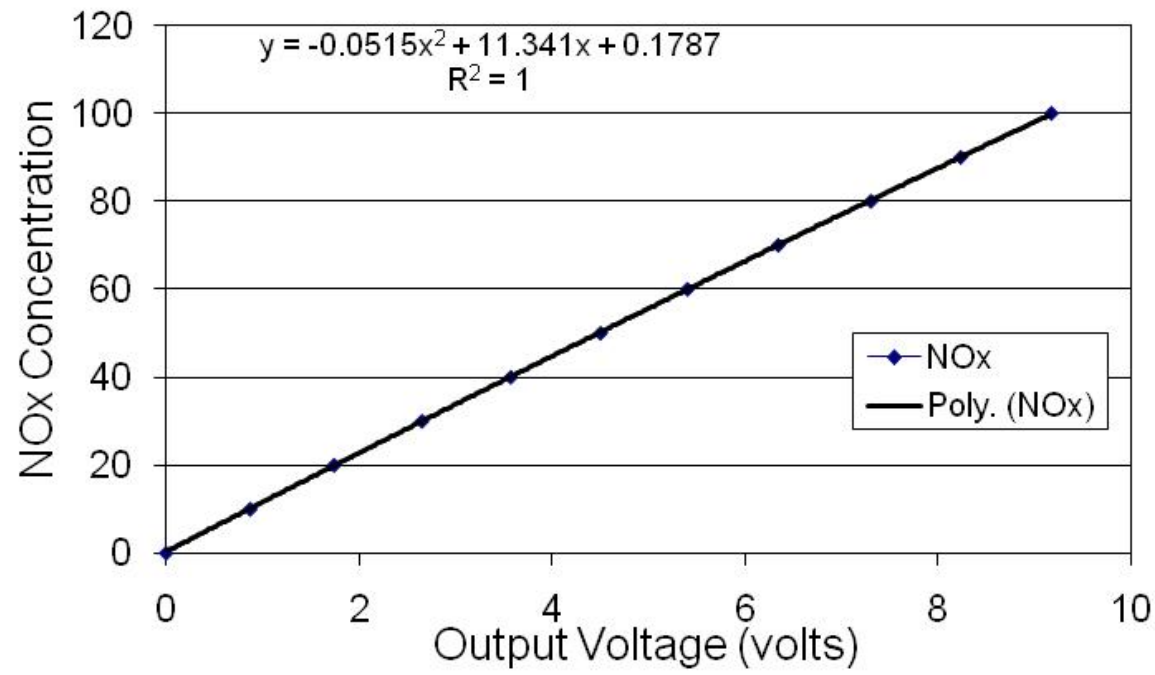

Figure 7-3: Calibration polynomial regression equation for NOx analyzer. 


\section{Appendix B: Engine power calculation and torque curve}

In order to obtain engine power, it was calculated using the vehicle's engine torque curve and the recorded engine parameters: engine speed and calculated percentage load. Using the recorded engine speed, the maximum torque the engine could produce at that engine speed, shown at numerous engine speeds in Table 8-1 and plotted in Figure 8-1, was determined by interpolation. The actual torque of the engine was then determined by multiplying this interpolated maximum torque value with the recorded calculated percentage load. The power was then calculated using Equation 3:

$$
\text { Power }=\frac{\text { Torque } \cdot \text { EngineSpeed }}{5252}
$$

where the power is in units of horsepower, the torque is in units of $\mathrm{ft}-\mathrm{lbs}$, engine speed is in units of revolutions per minute (RPM), and 5252 is a conversion factor.

Table 8-1: Torque vs. engine speed for Dodge Ram 2500.

\begin{tabular}{|c|c|}
\hline Speed & Torque \\
\hline$(\mathrm{rpm})$ & $(\mathrm{lb}-\mathrm{ft})$ \\
\hline 0 & 0 \\
\hline 200 & 96.25 \\
\hline 400 & 192.5 \\
\hline 600 & 288.75 \\
\hline 800 & 385 \\
\hline 1000 & 440 \\
\hline 1200 & 497 \\
\hline 1400 & 555 \\
\hline 1600 & 610 \\
\hline 1800 & 607 \\
\hline 2000 & 604 \\
\hline 2200 & 600 \\
\hline 2400 & 597 \\
\hline 2600 & 594 \\
\hline 2800 & 591 \\
\hline 2900 & 589 \\
\hline 3000 & 491 \\
\hline 3100 & 393 \\
\hline 3200 & 295 \\
\hline 3500 & 0 \\
\hline
\end{tabular}




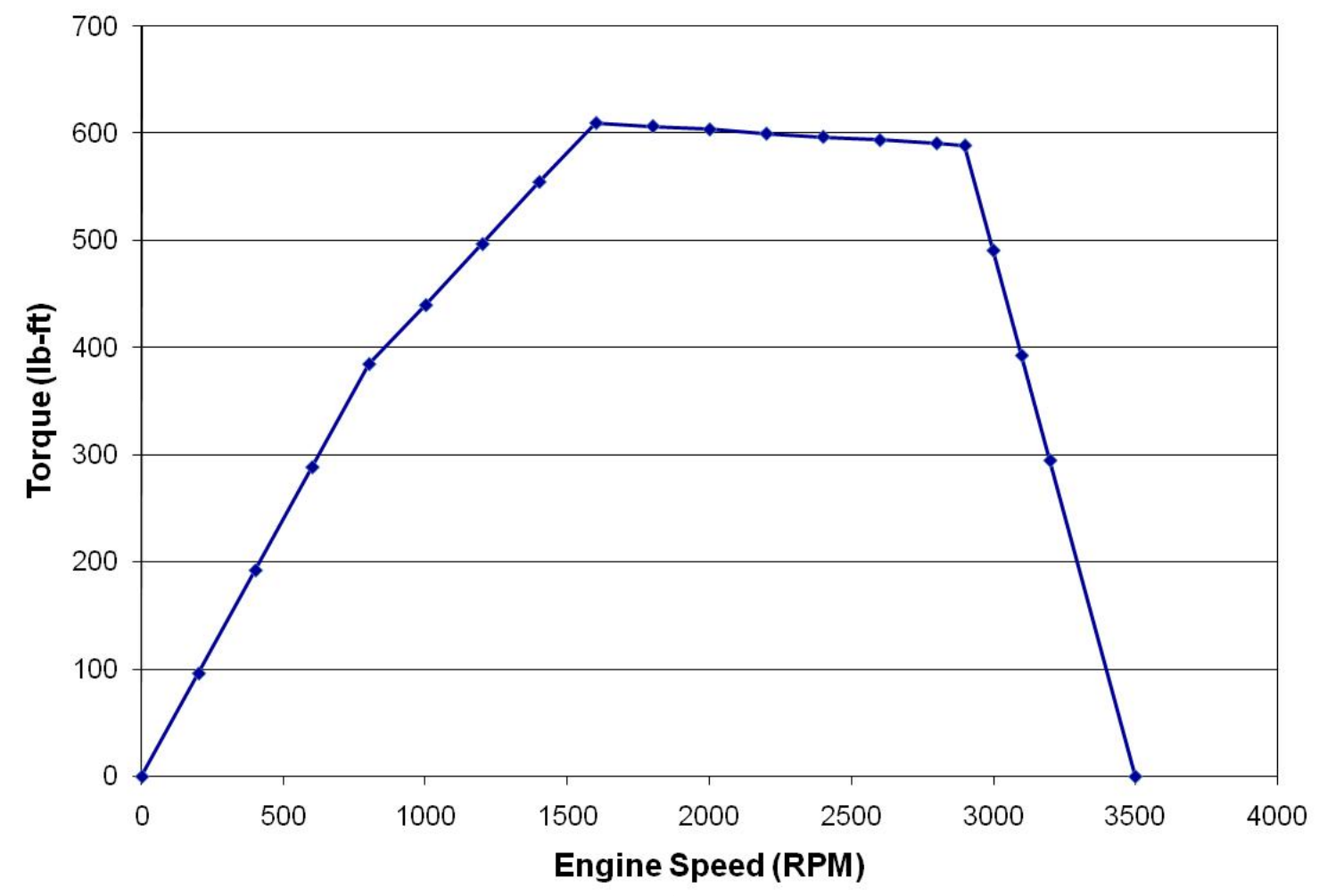

Figure 8-1: Torque curve for the Dodge Ram 2500. 


\section{Appendix C: Drive-by particle and mass concentrations}

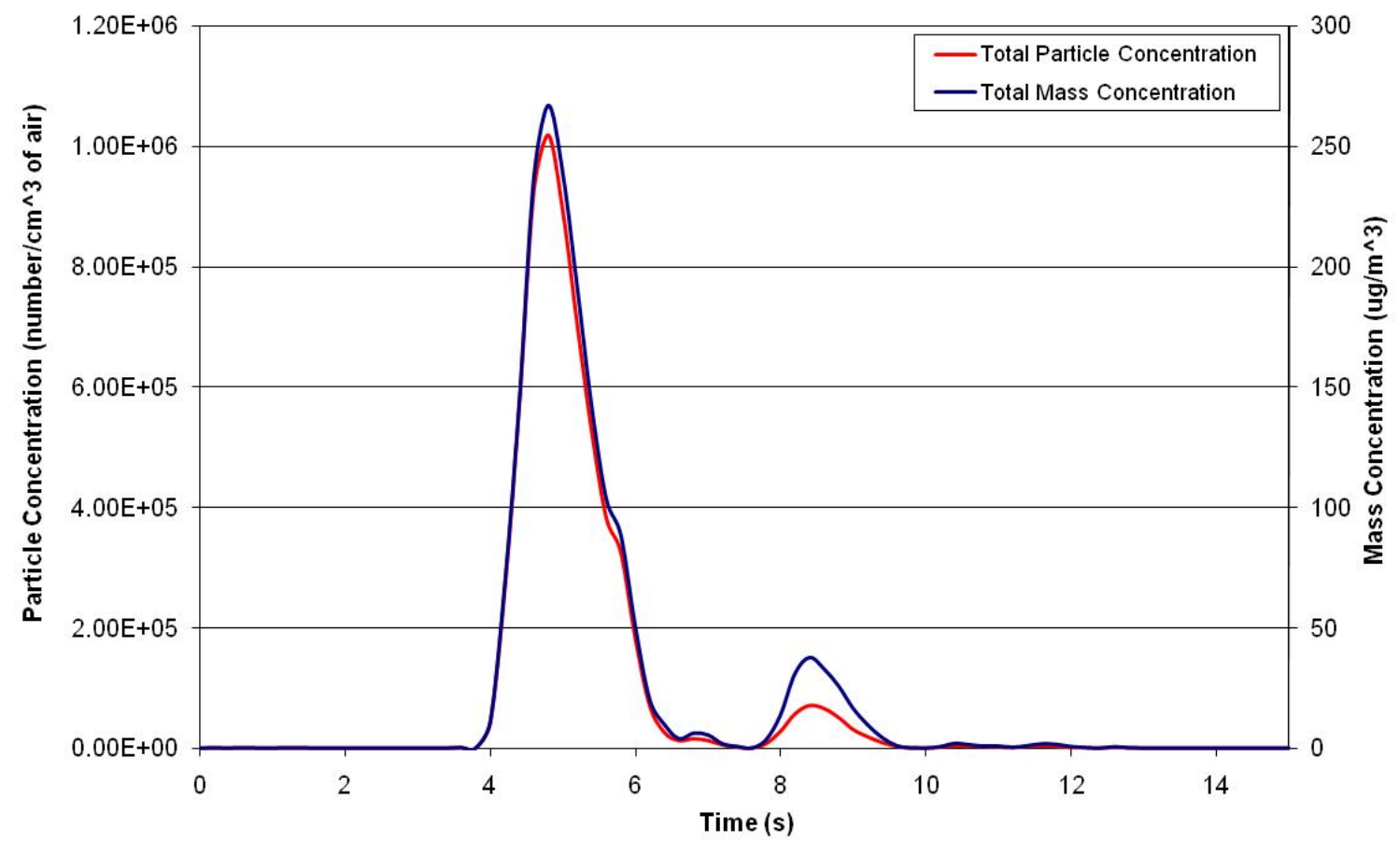

Figure 9-1: Hard acceleration diesel drive-by with mannequin 1.

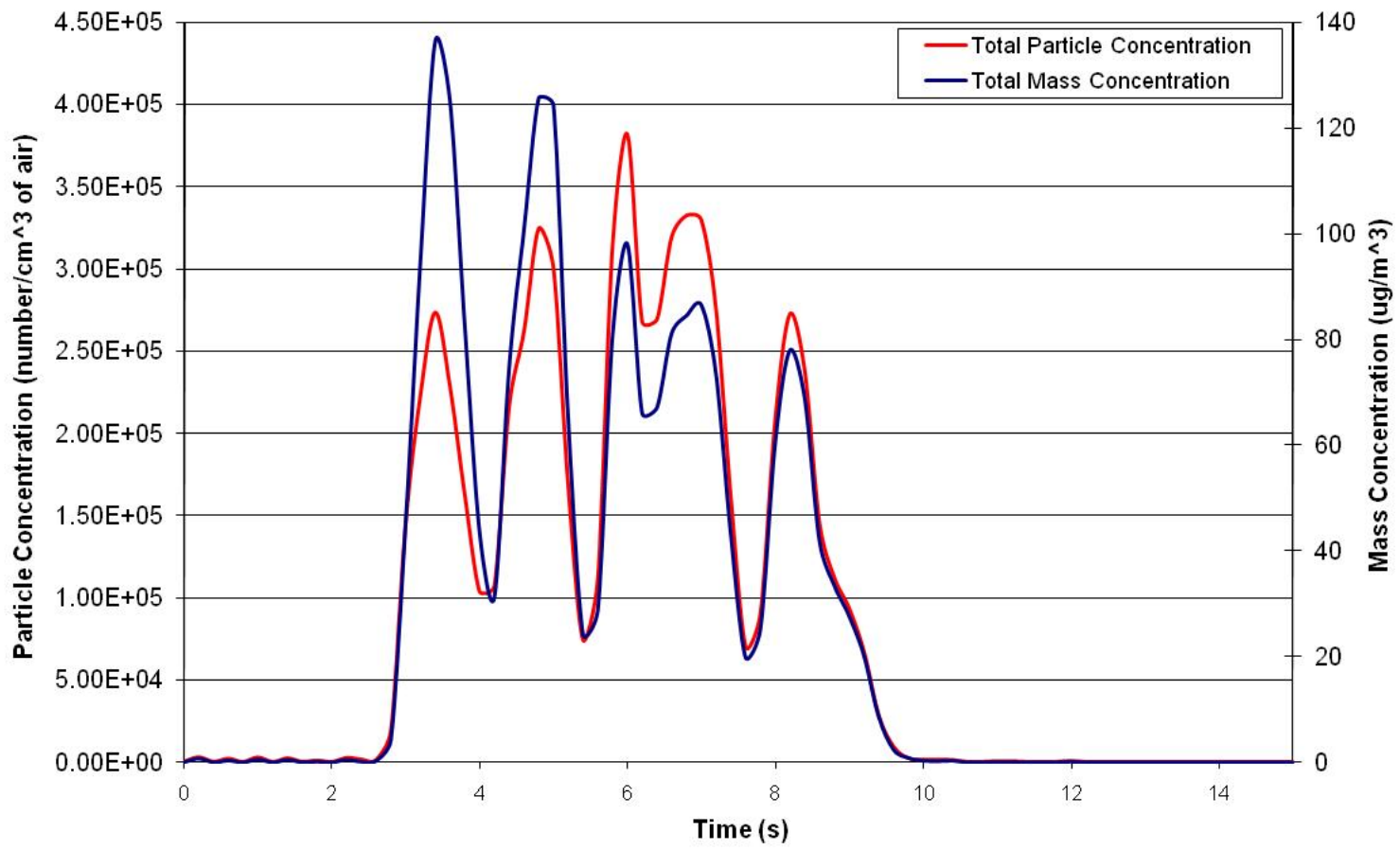

Figure 9-2: Hard acceleration diesel drive-by with mannequin 2. 


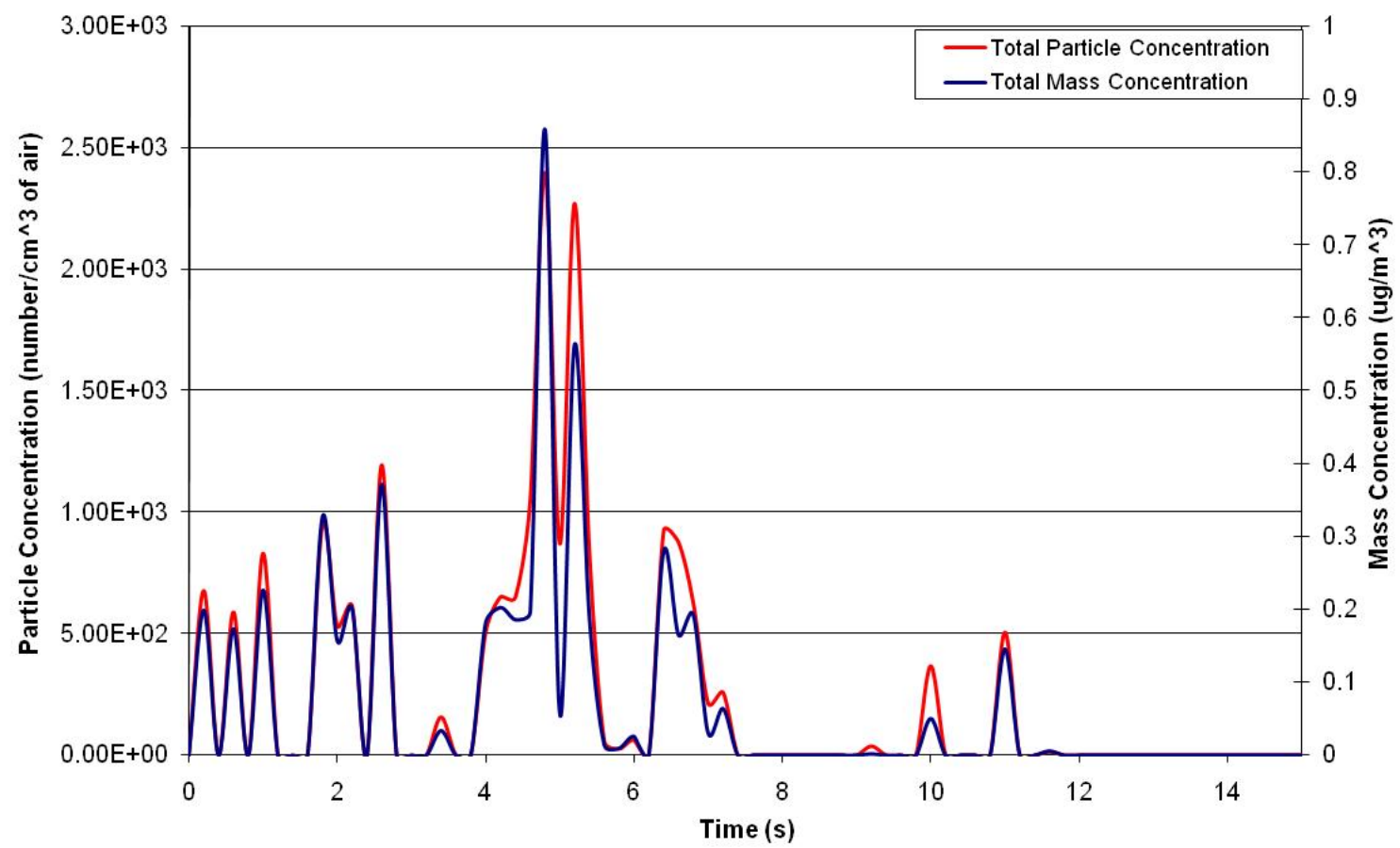

Figure 9-3: Hard acceleration diesel drive-by with mannequin 3.

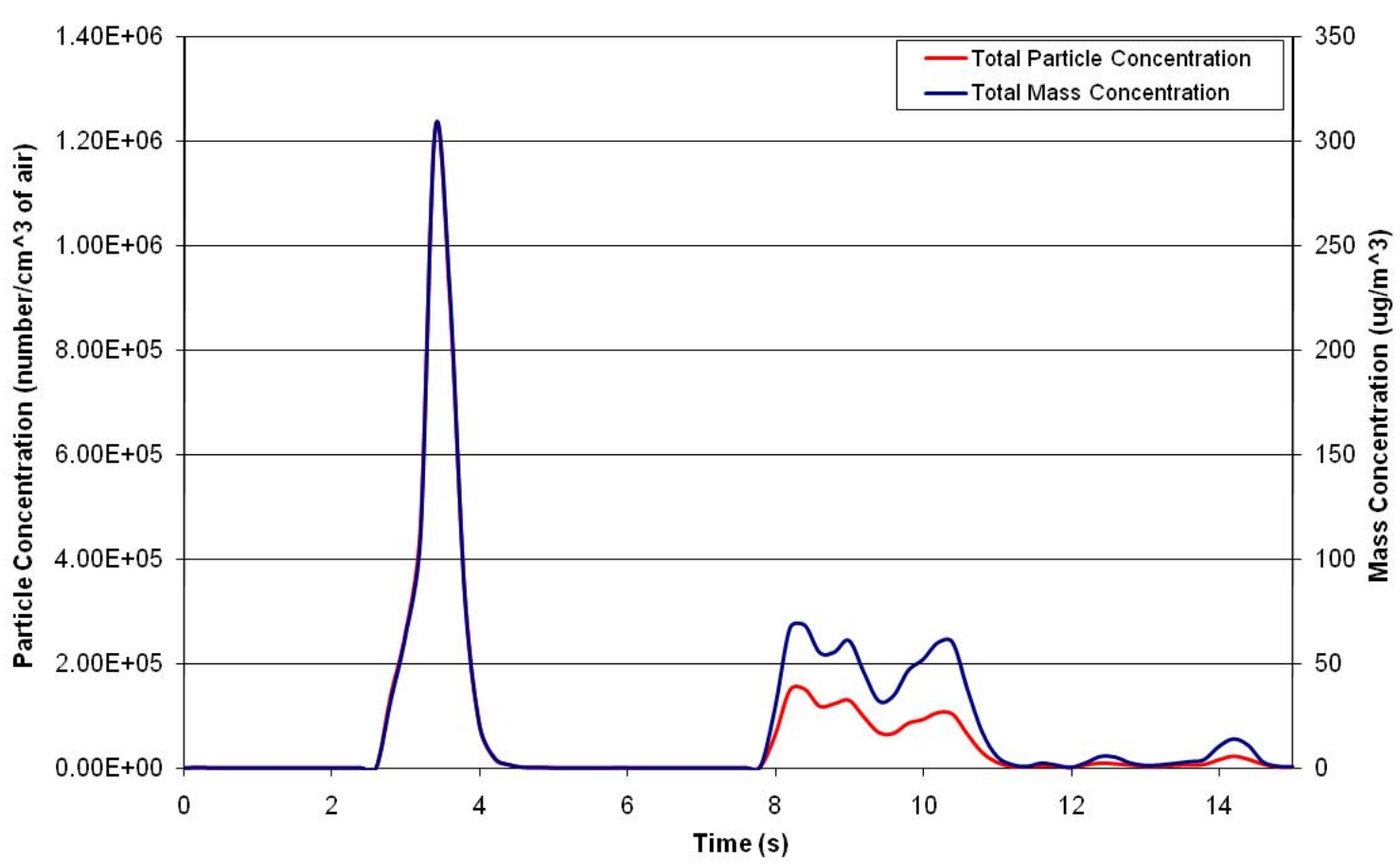

Figure 9-4: Hard acceleration diesel drive-by with mannequin 4. 


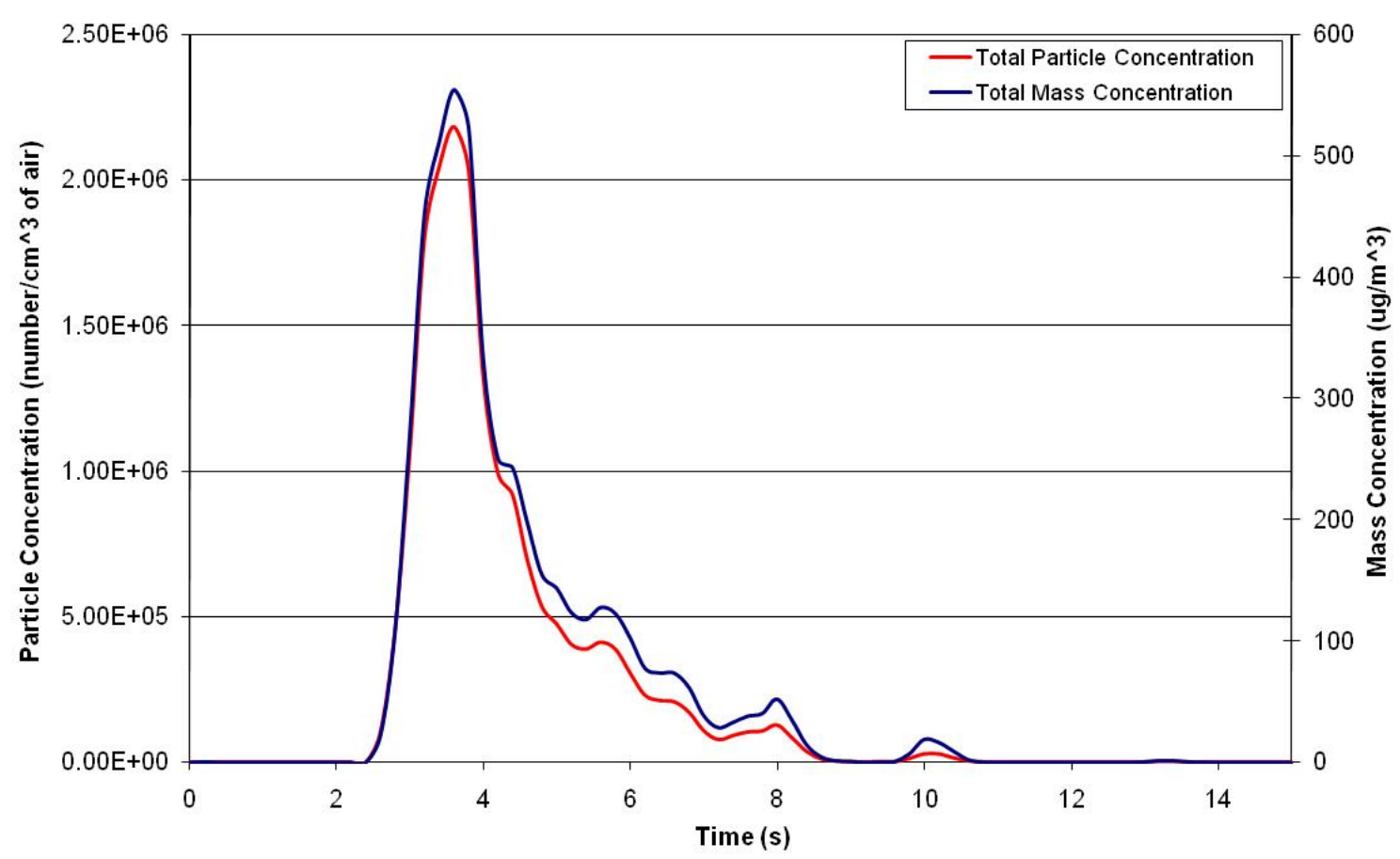

Figure 9-5: Hard acceleration diesel drive-by with mannequin 5.

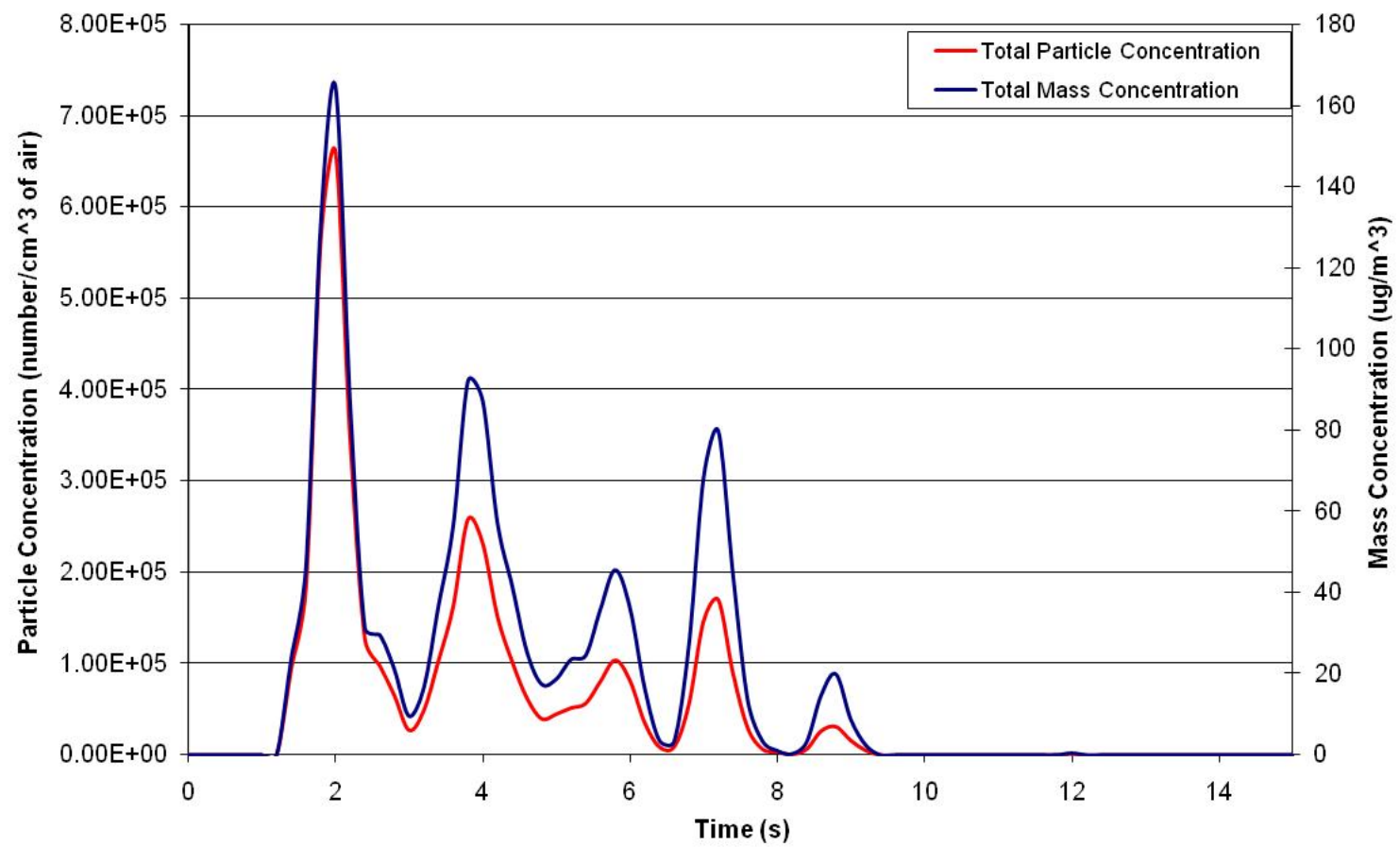

Figure 9-6: Hard acceleration diesel drive-by with mannequin 6. 


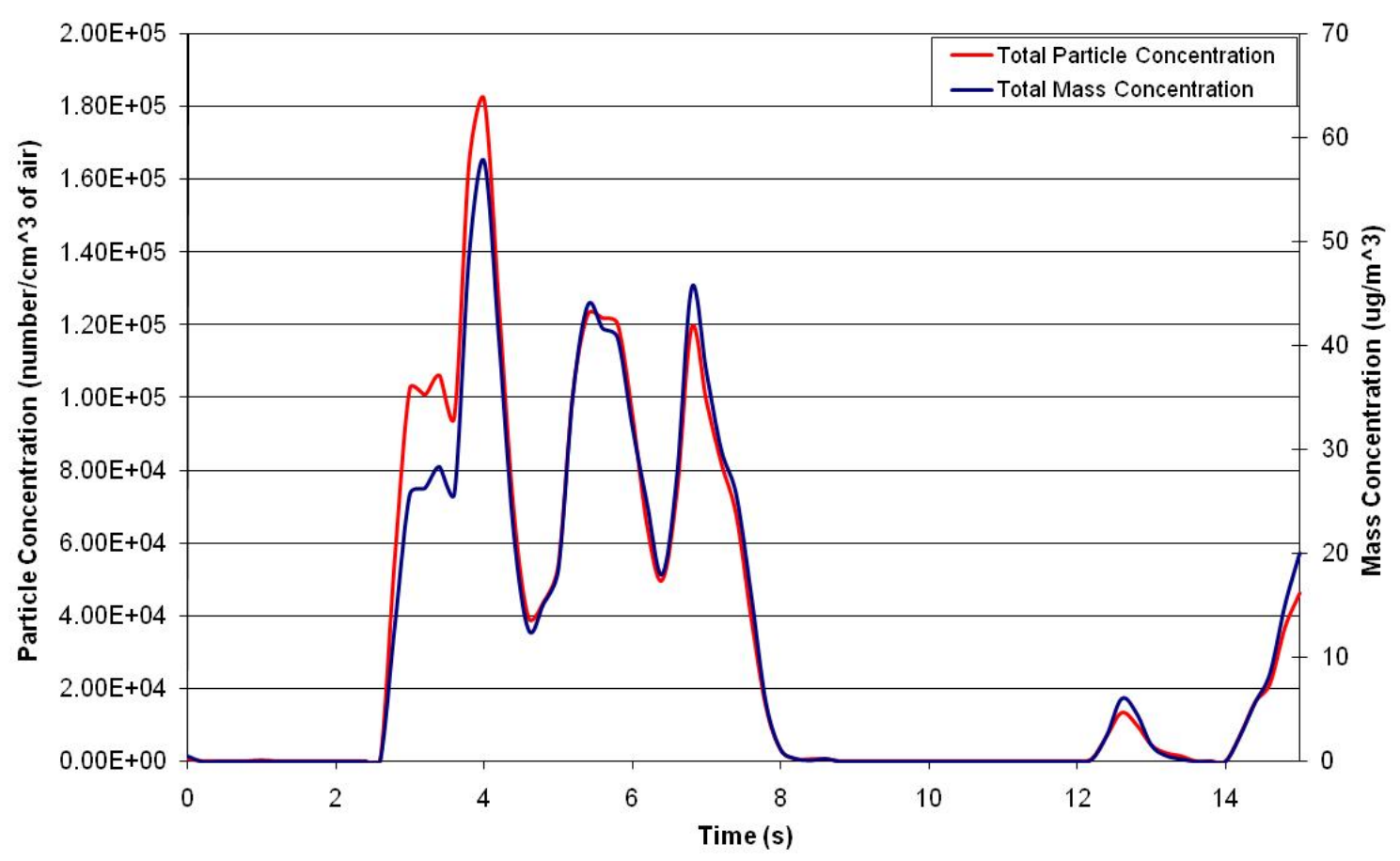

Figure 9-7: Medium acceleration diesel drive-by with mannequin 1.

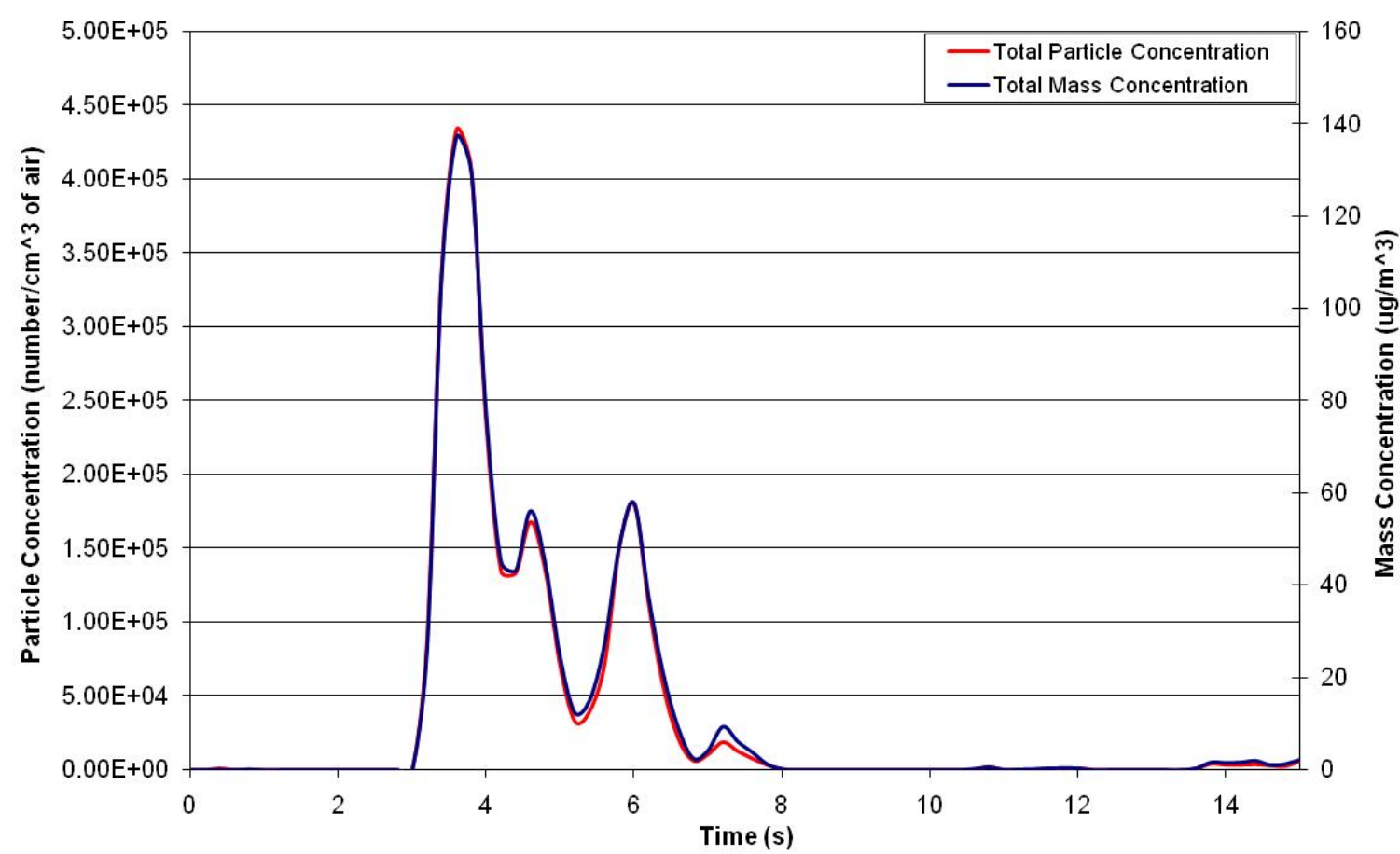

Figure 9-8: Medium acceleration diesel drive-by with mannequin 2. 


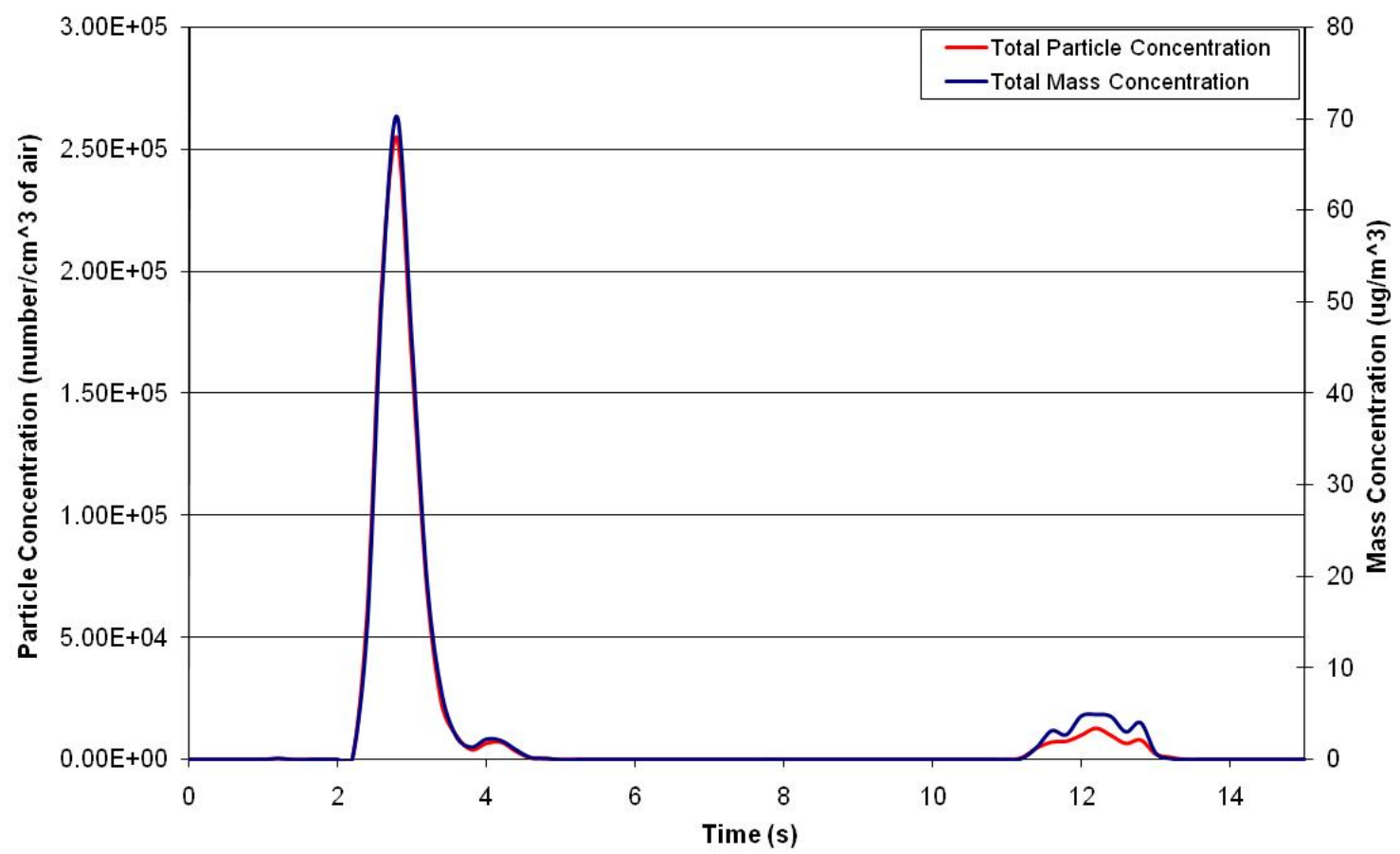

Figure 9-9: Medium acceleration drive-by with mannequin 3.

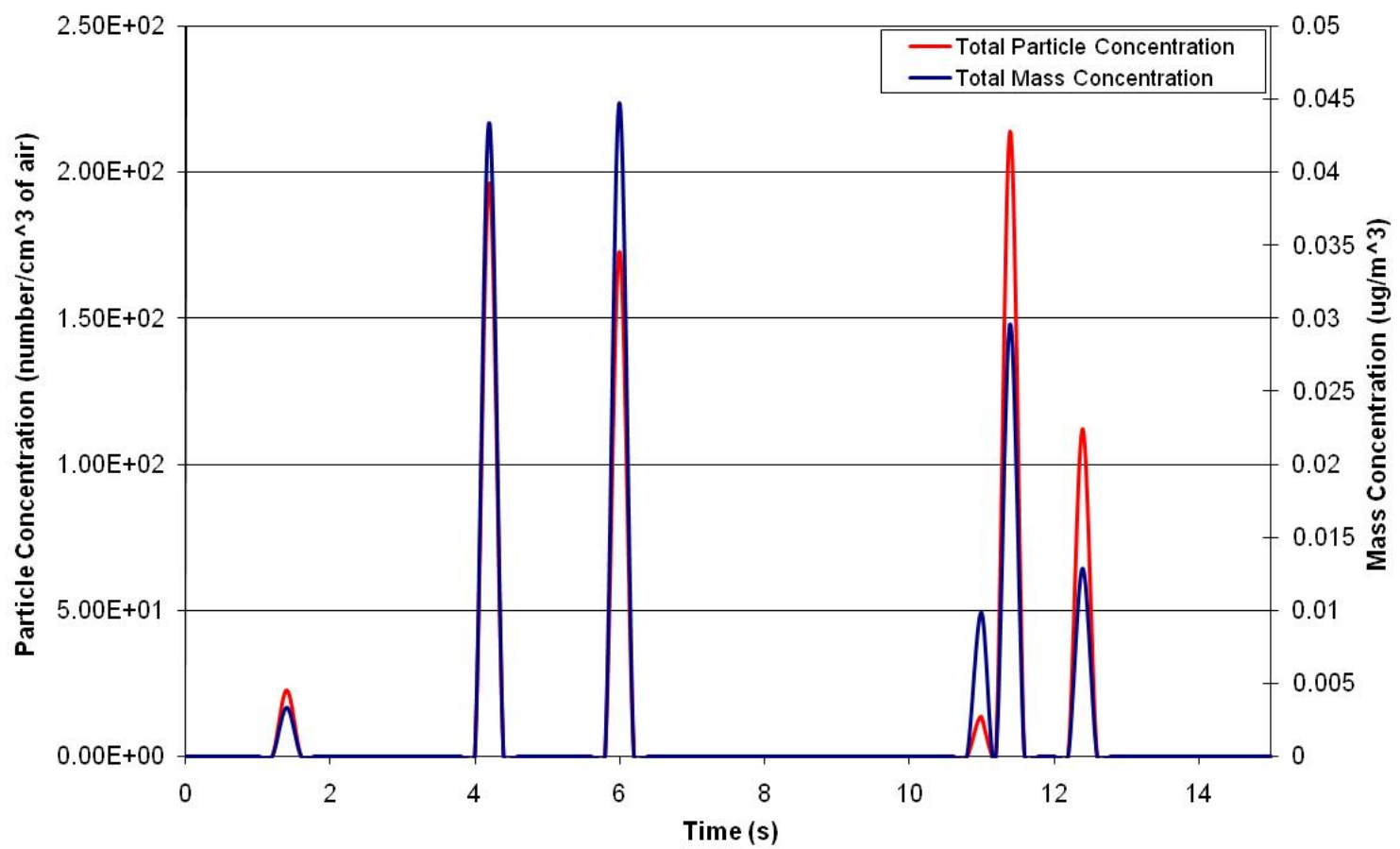

Figure 9-10: Medium acceleration diesel drive-by with mannequin 4. 


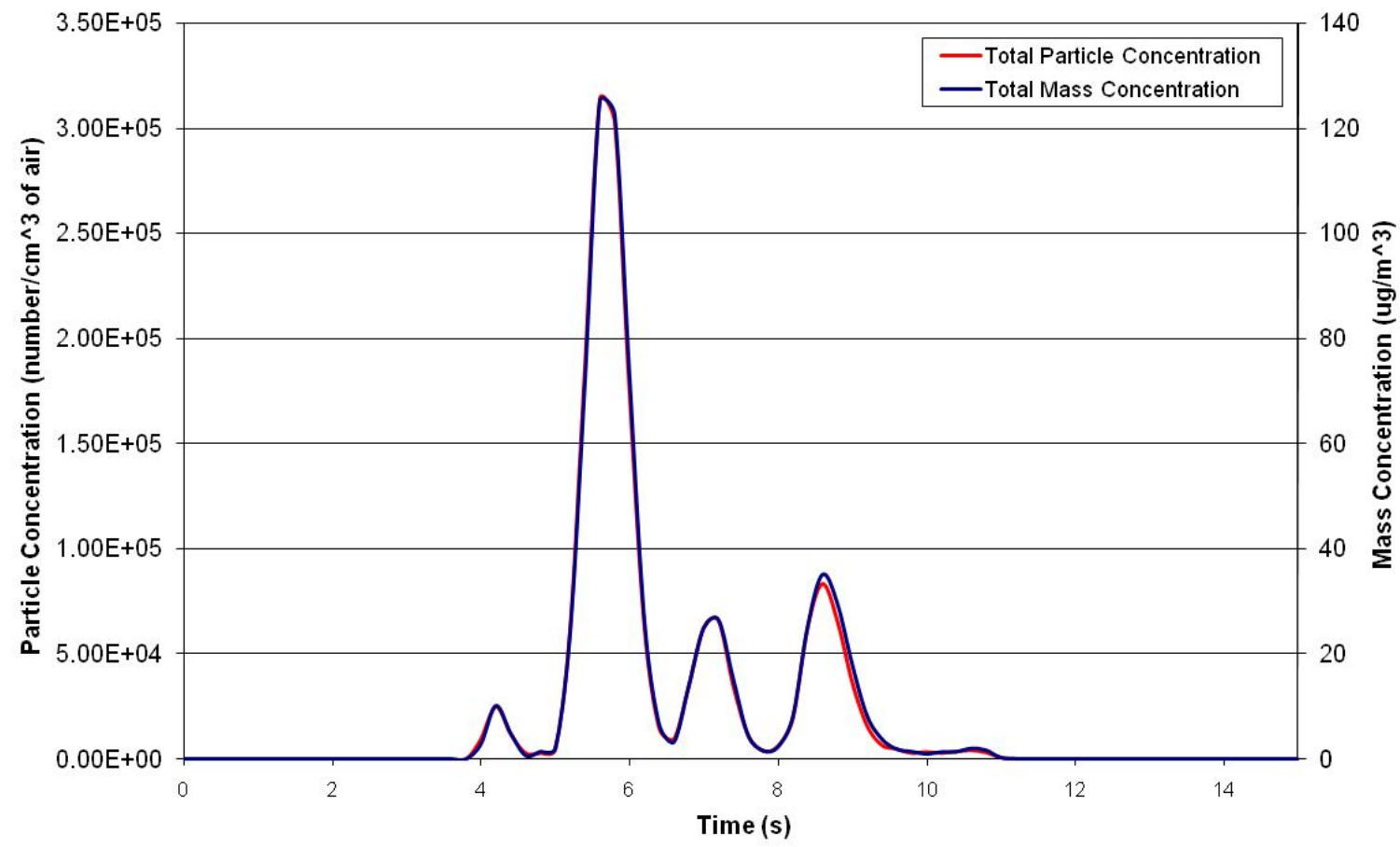

Figure 9-11: Medium acceleration diesel drive-by with mannequin 5.

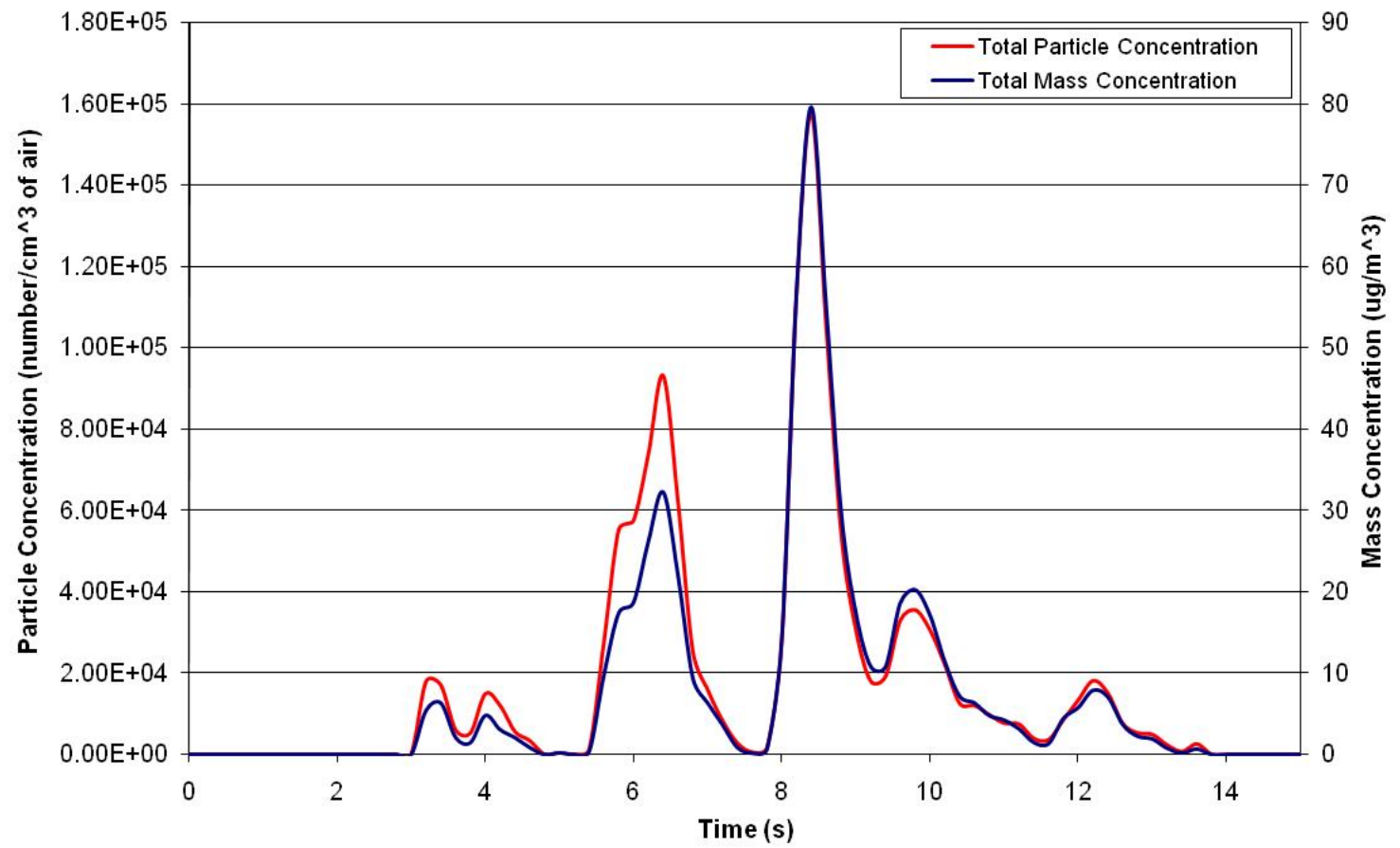

Figure 9-12: Medium acceleration diesel drive-by with mannequin 6. 


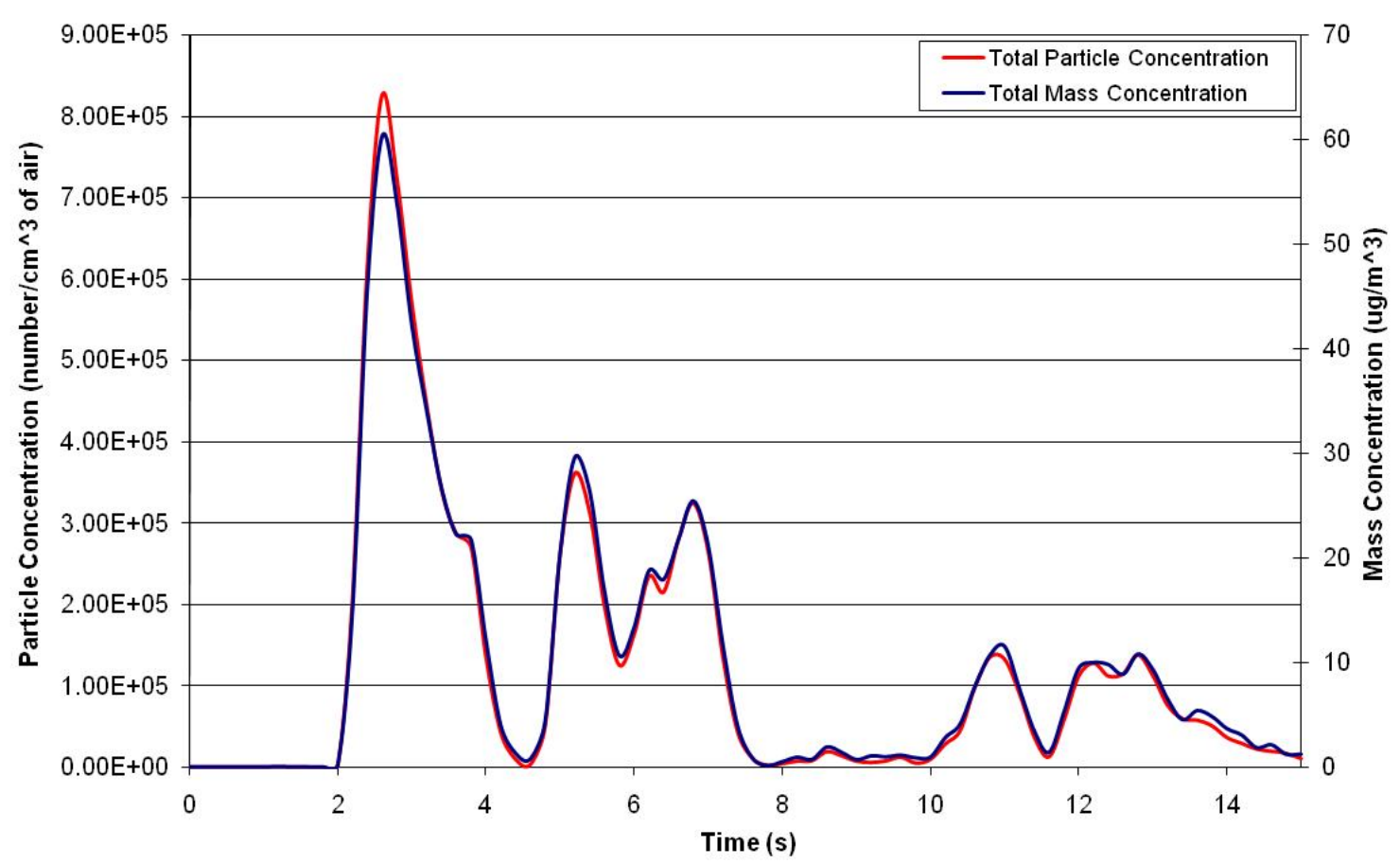

Figure 9-13: Cruising diesel drive-by with mannequin 1.

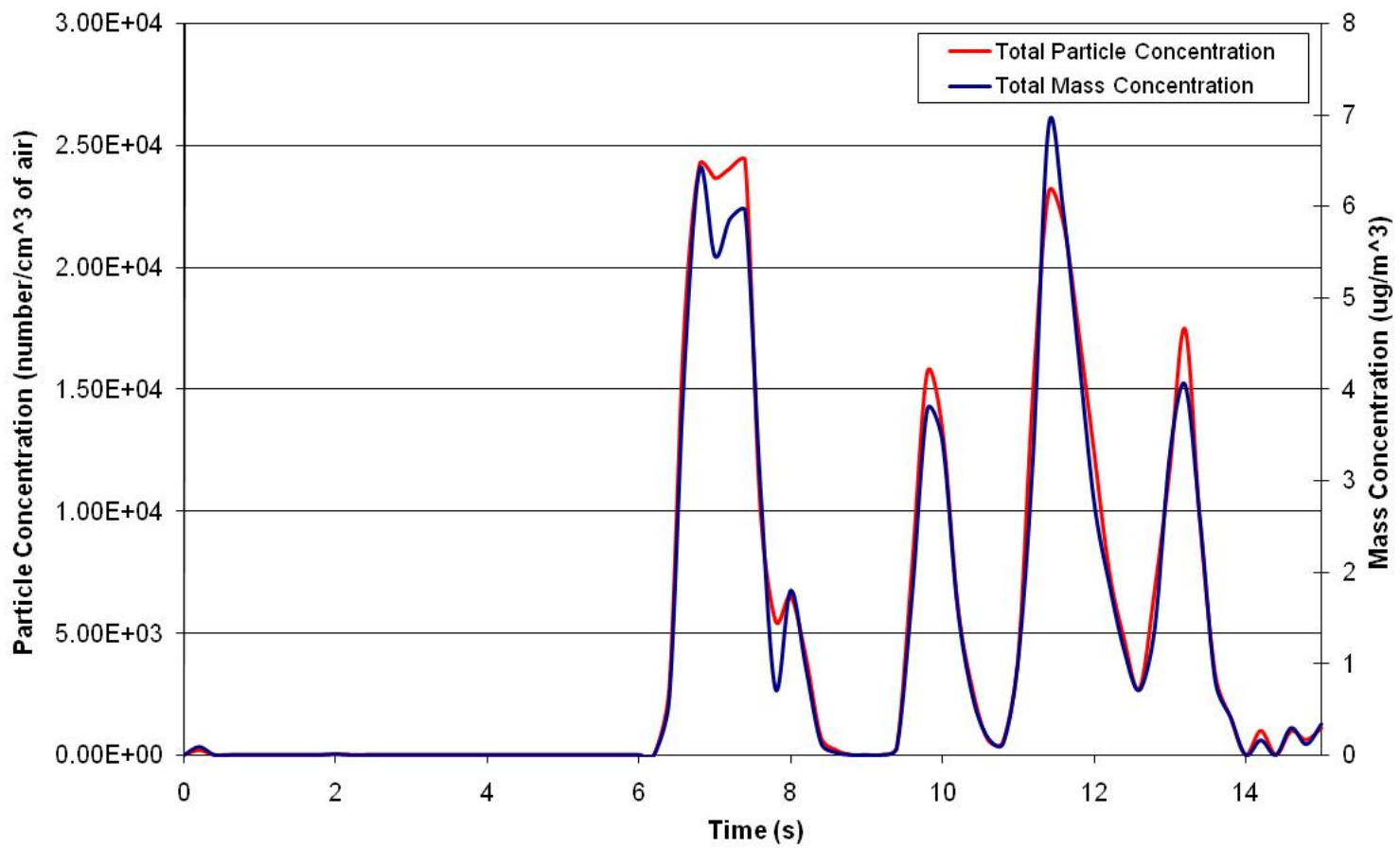

Figure 9-14: Cruising diesel drive-by with mannequin 2. 


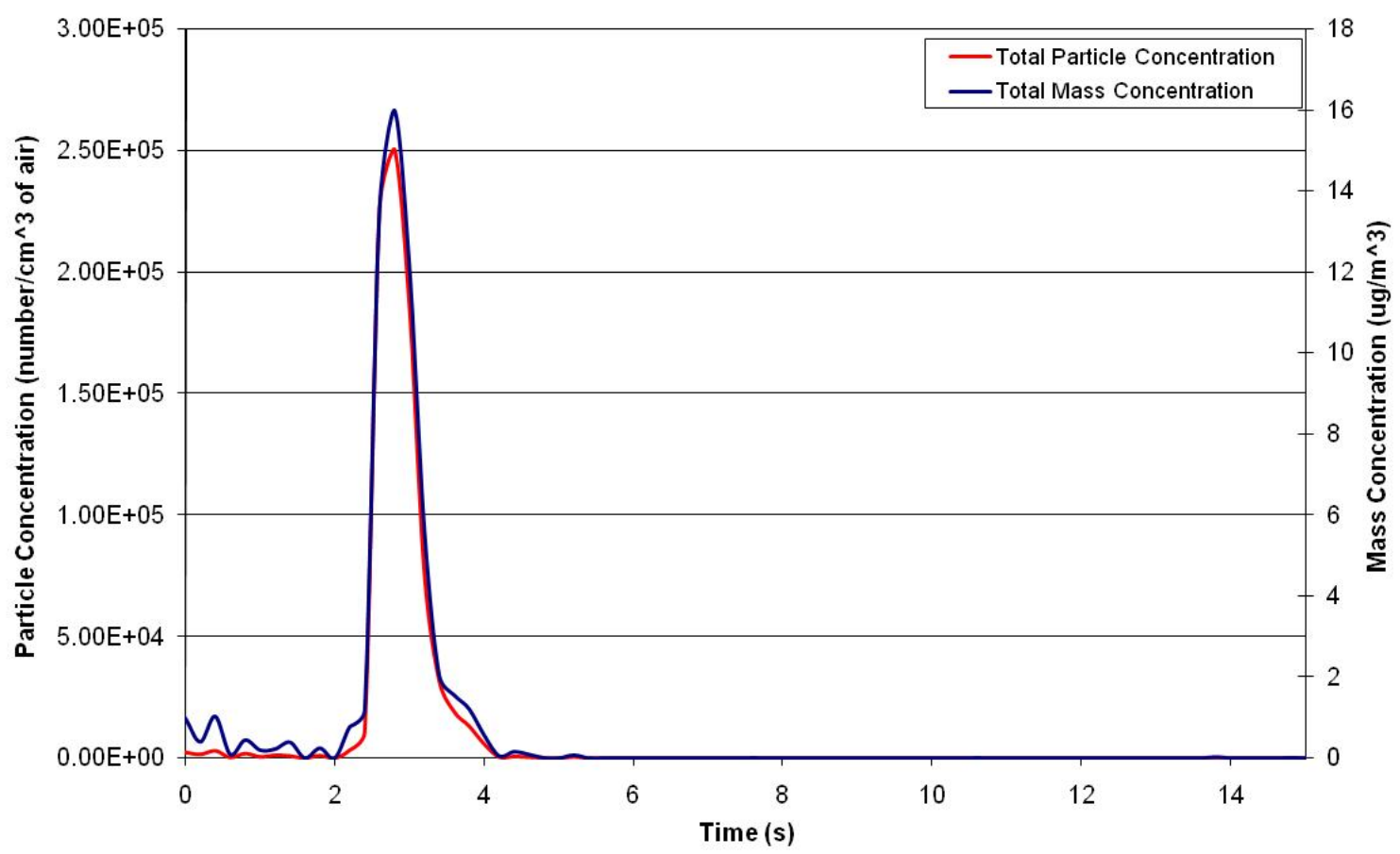

Figure 9-15: Cruising diesel drive-by with mannequin 3.

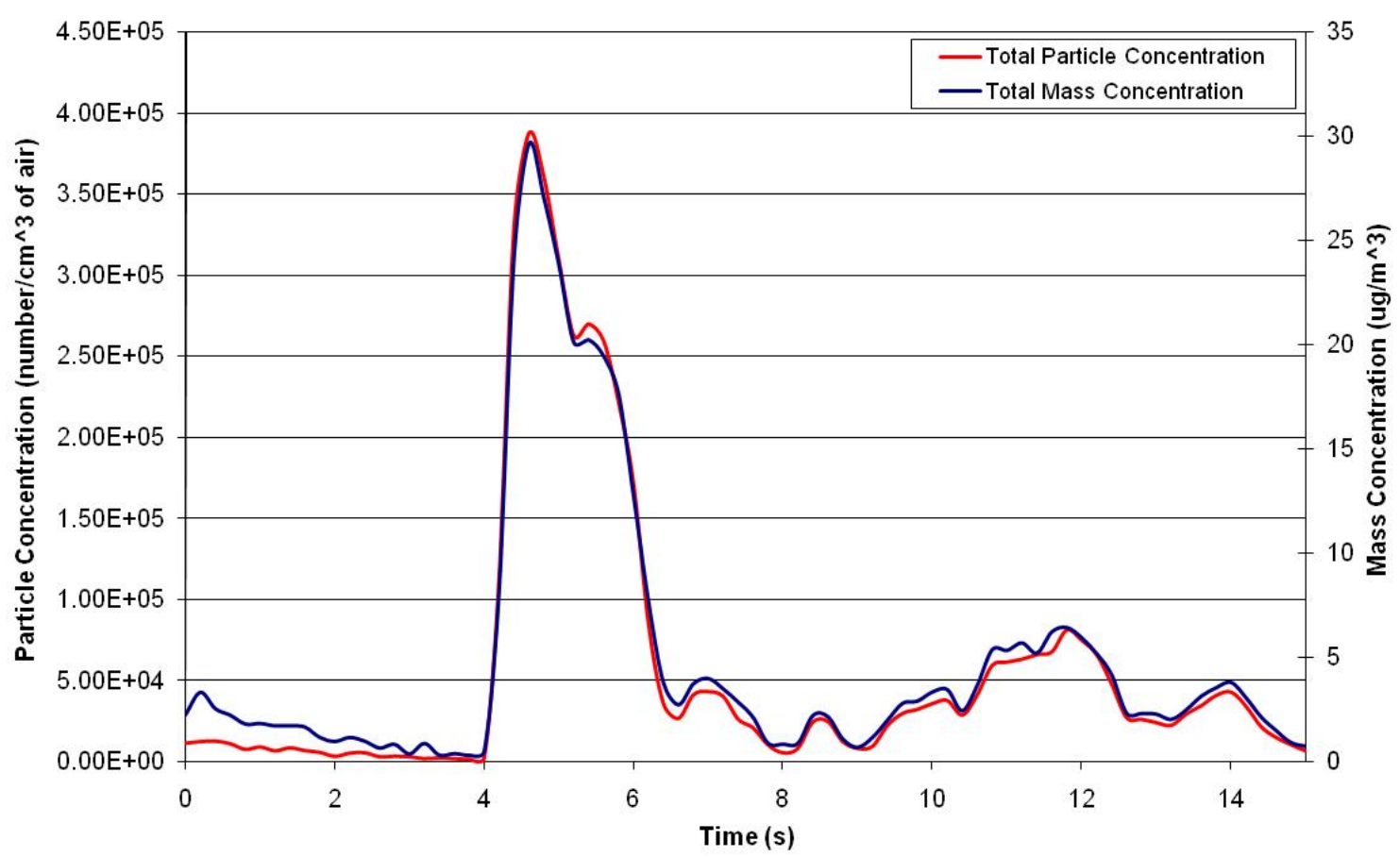

Figure 9-16: Cruising diesel drive-by with mannequin 4. 


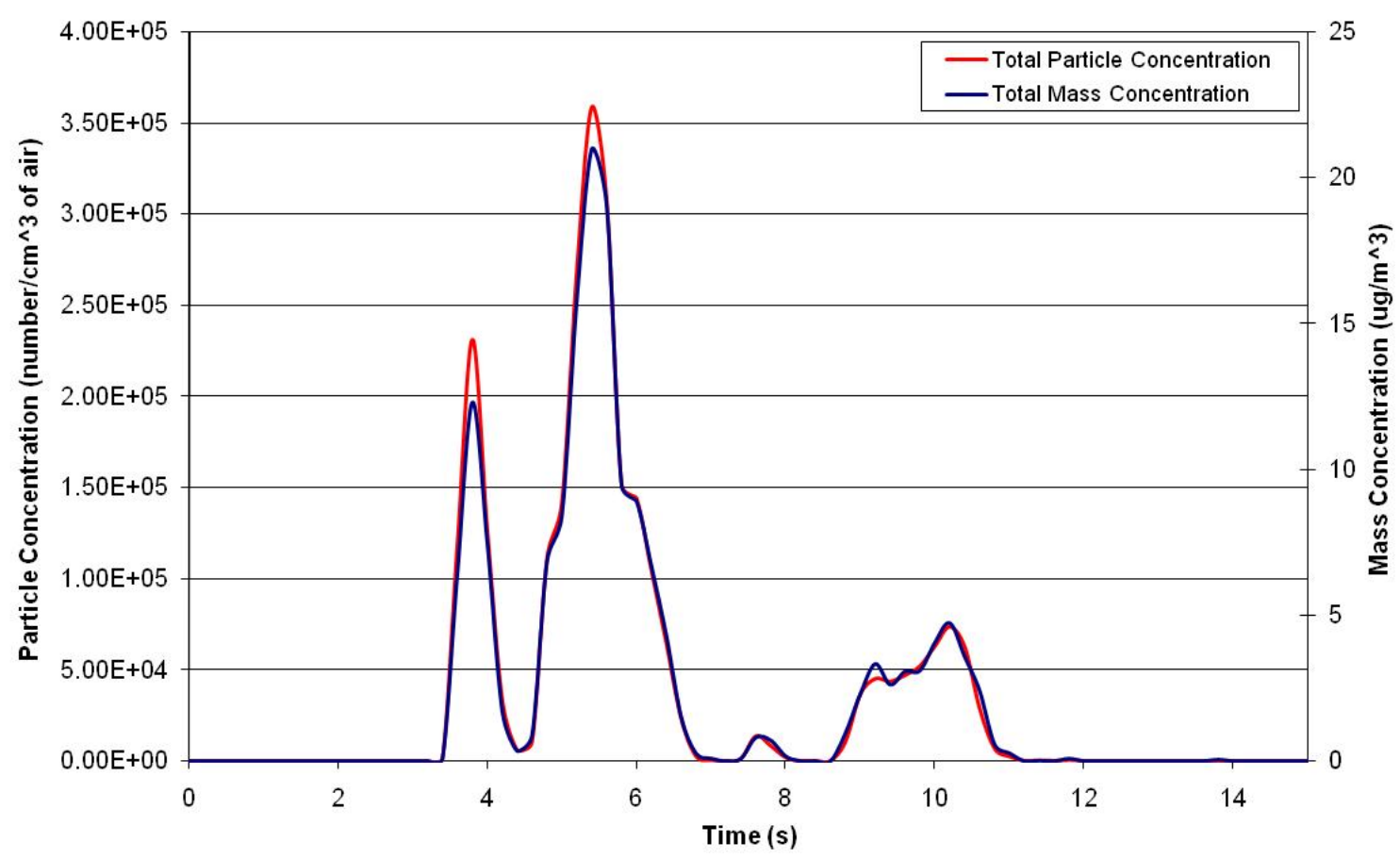

Figure 9-17: Cruising diesel drive-by with mannequin 5.

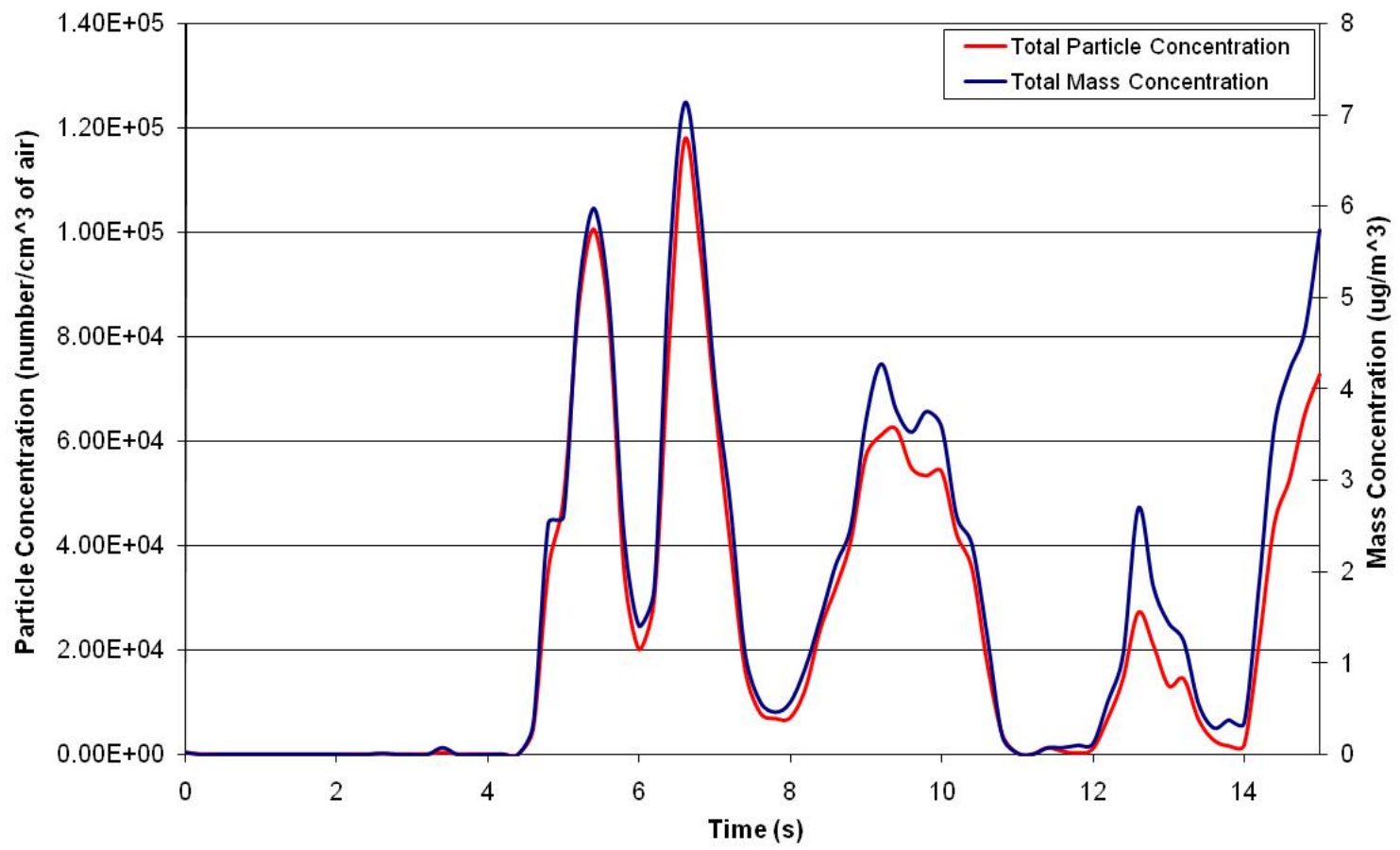

Figure 9-18: Cruising diesel drive-by with mannequin 6. 


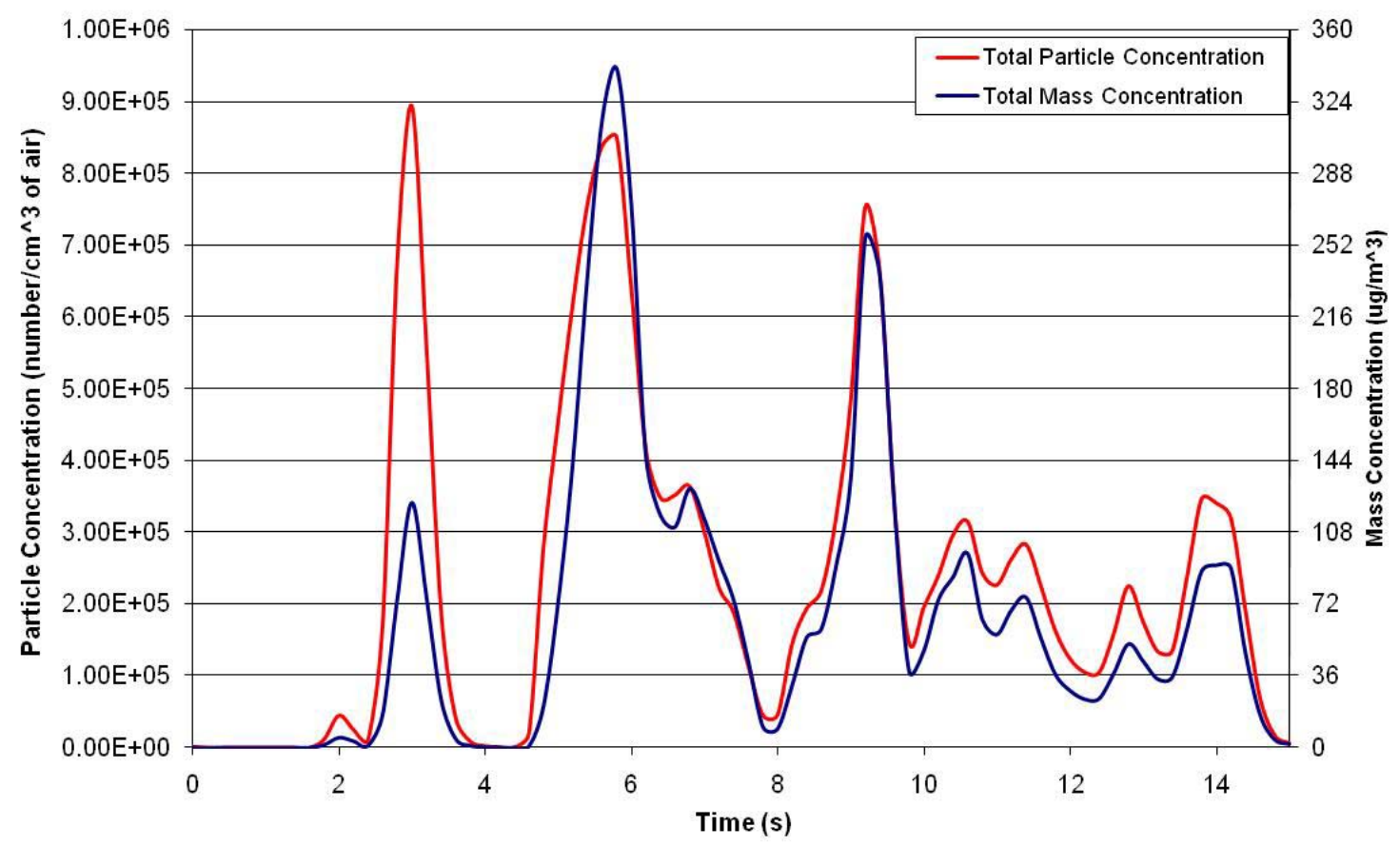

Figure 9-19: Hard acceleration diesel drive-by without mannequin 1.

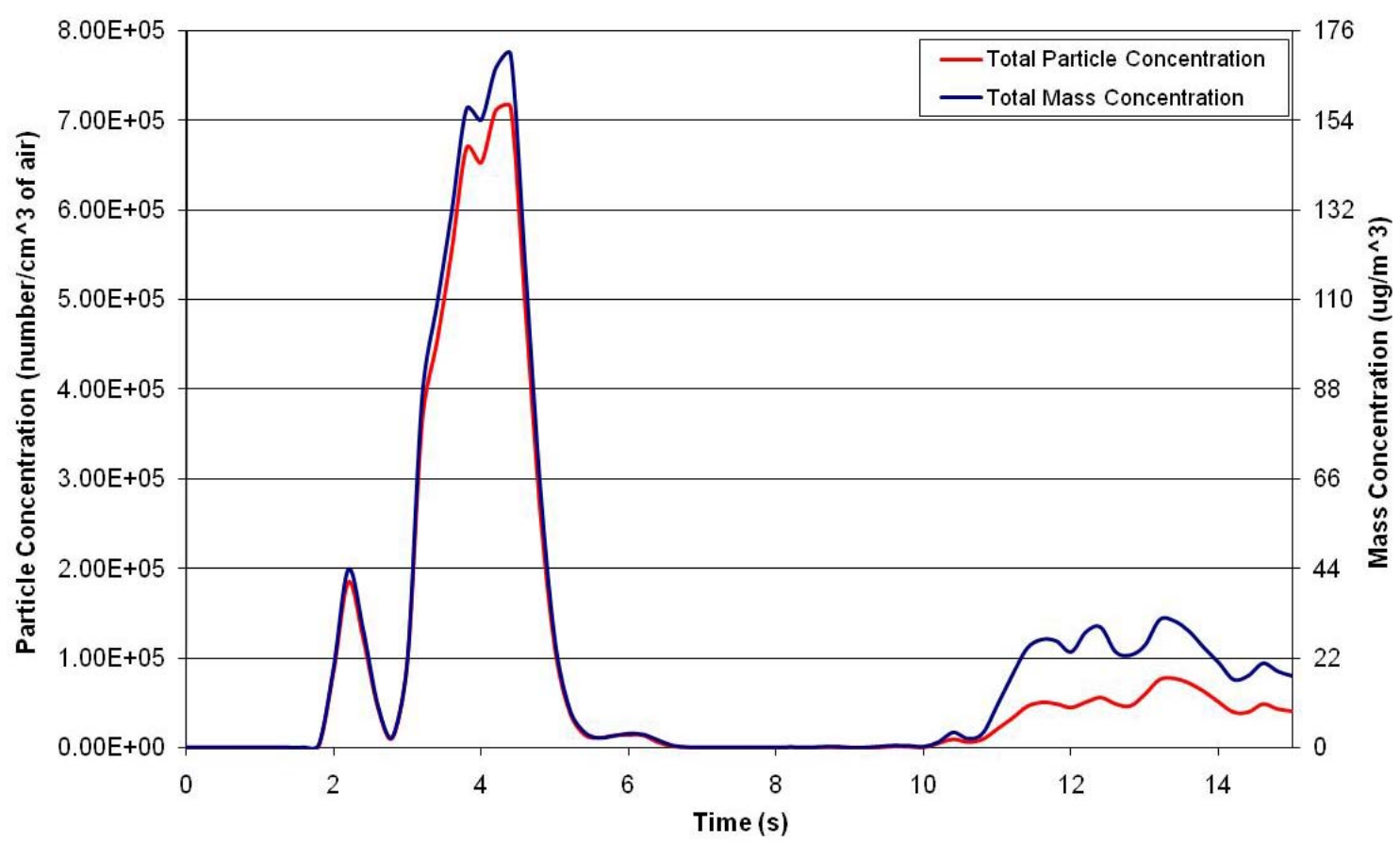

Figure 9-20: Hard acceleration diesel drive-by without mannequin 2. 


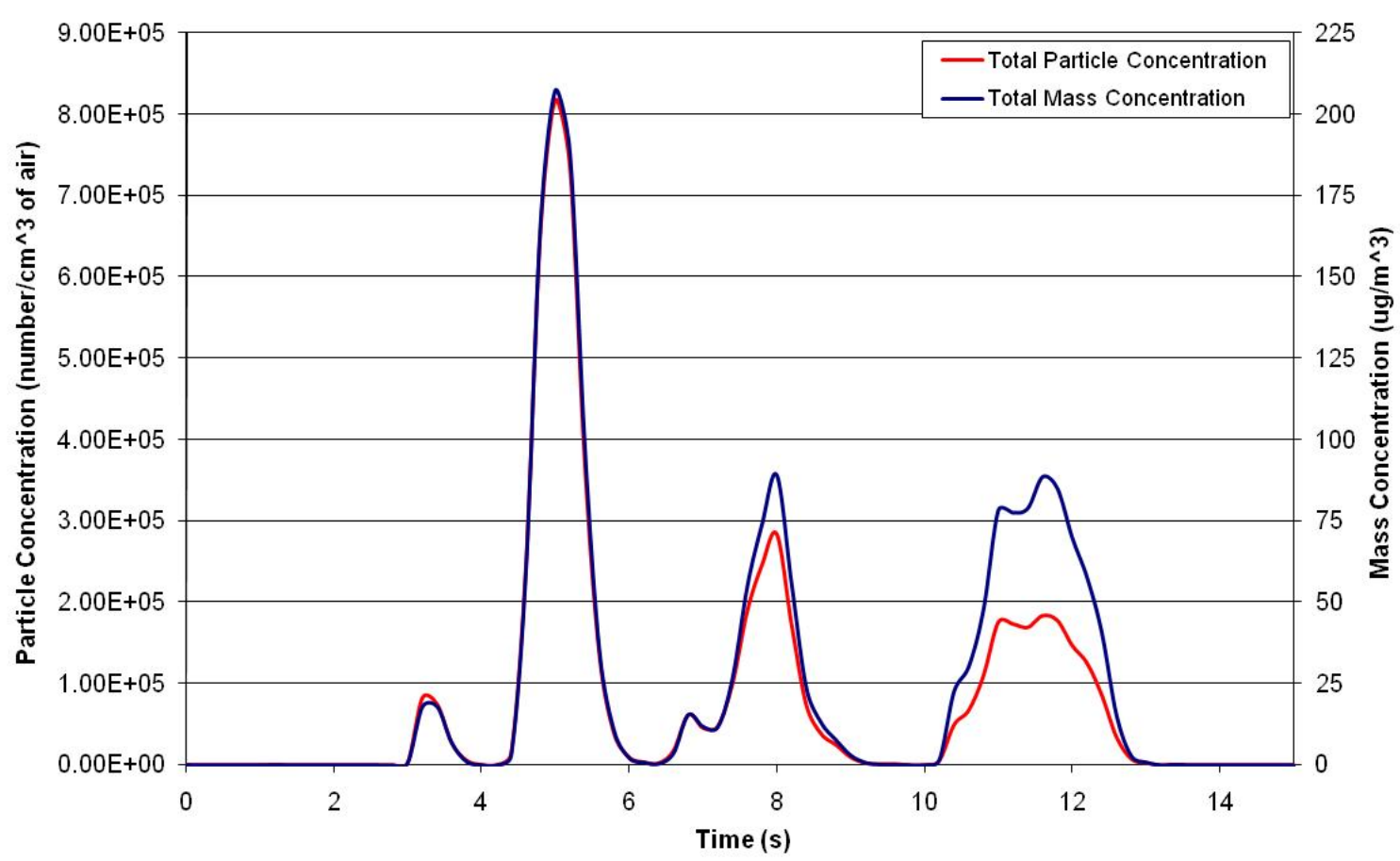

Figure 9-21: Hard acceleration diesel drive-by without mannequin 3.

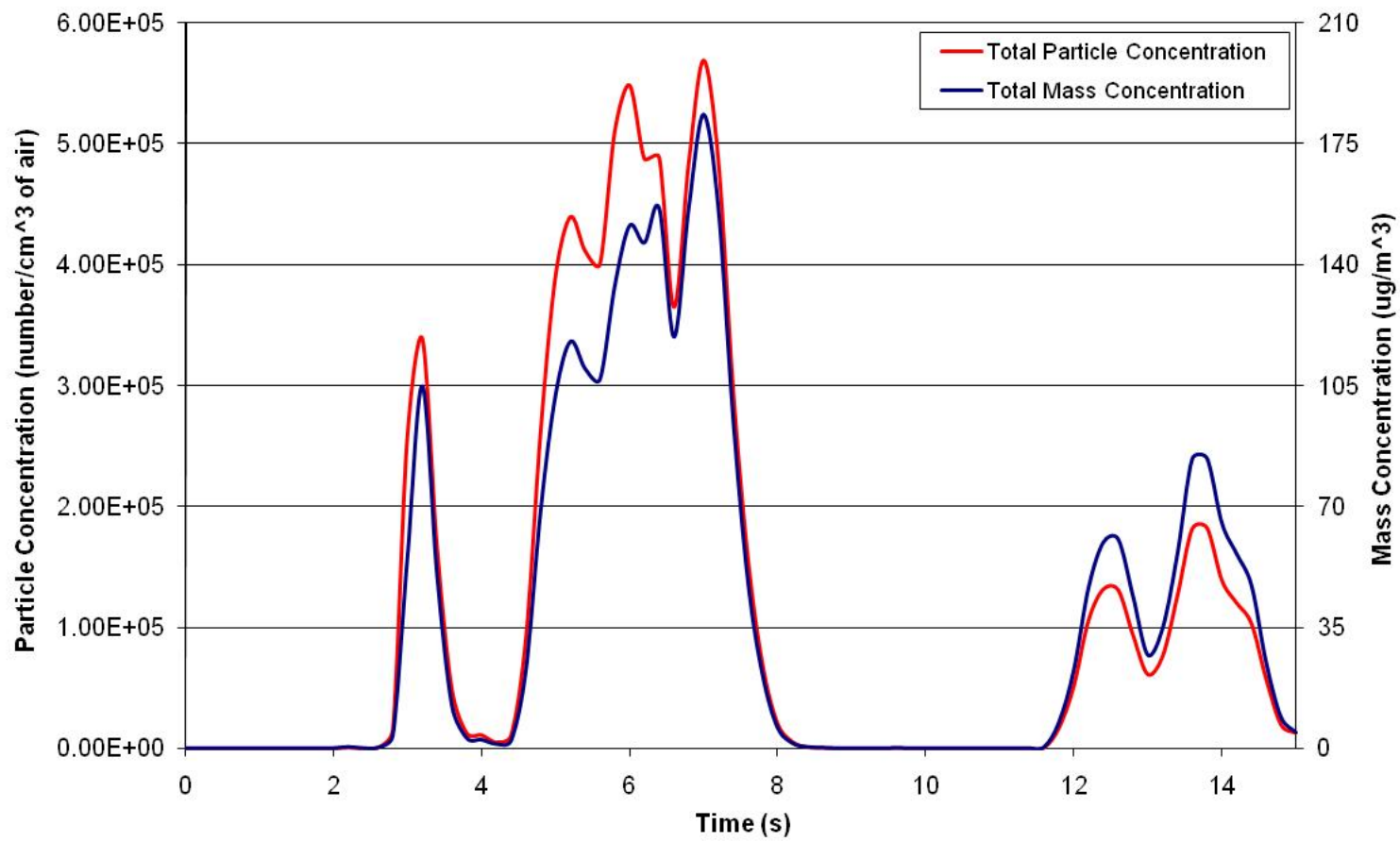

Figure 9-22: Hard acceleration diesel drive-by without mannequin 4. 


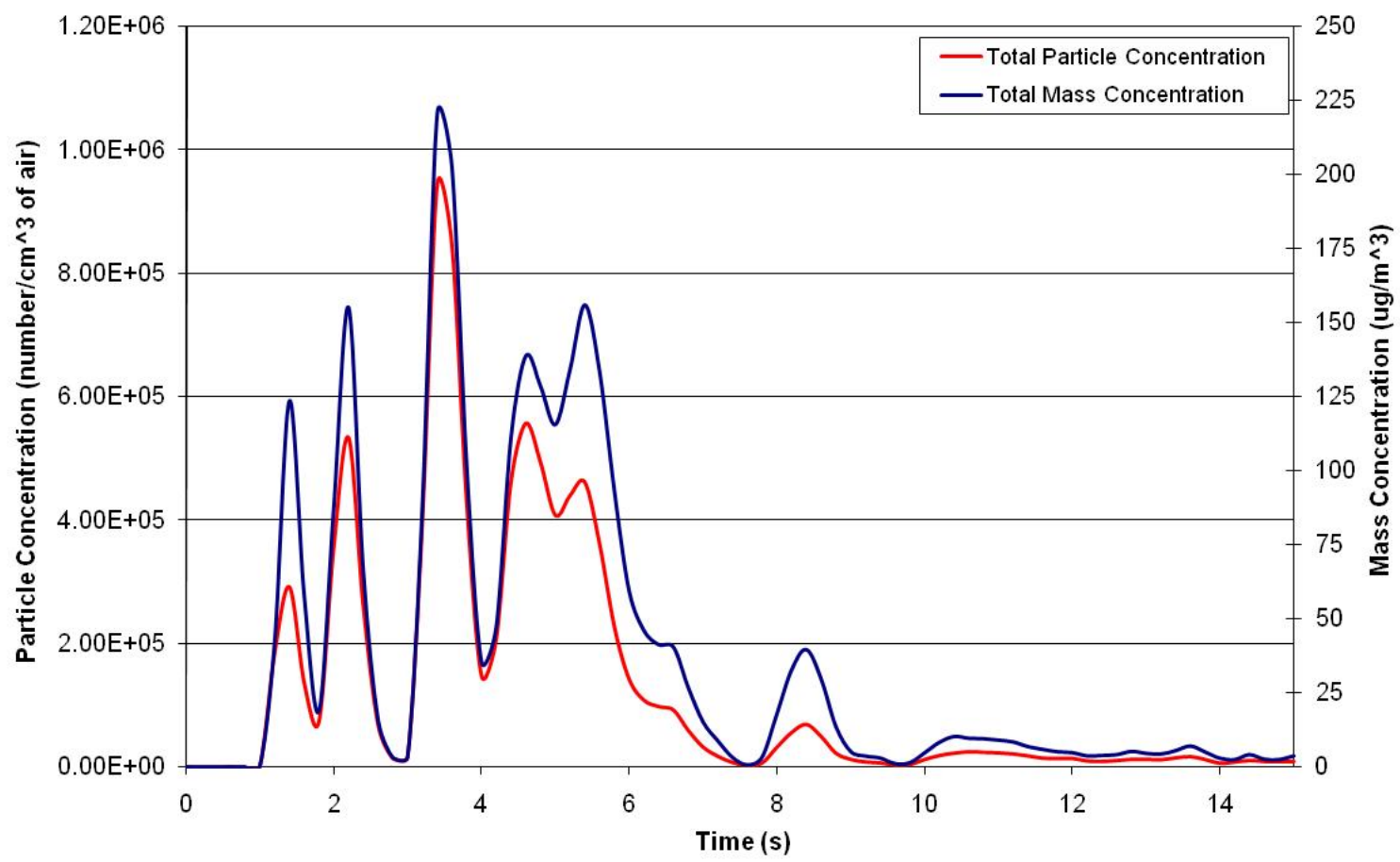

Figure 9-23: Hard acceleration diesel drive-by without mannequin 5.

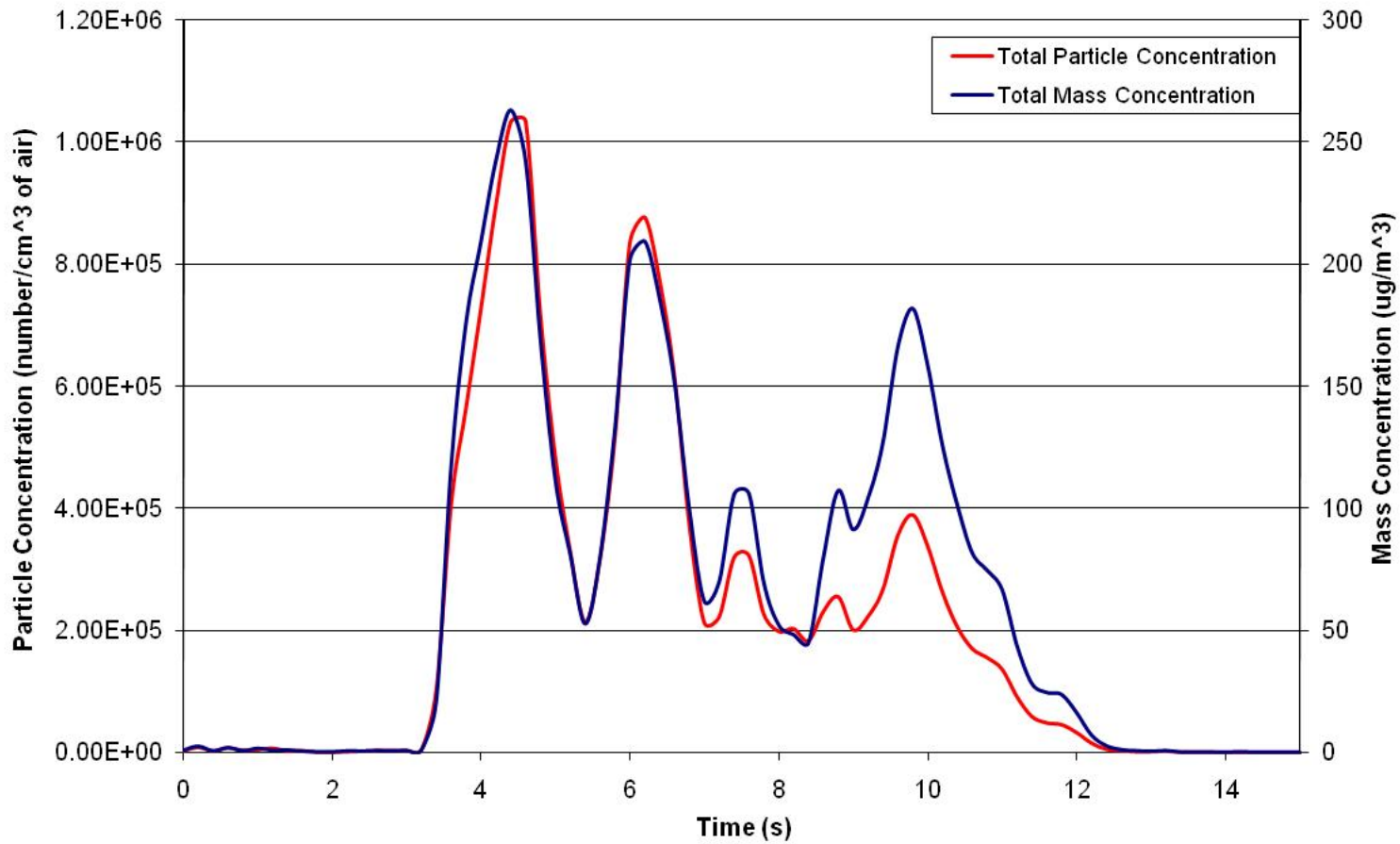

Figure 9-24: Hard acceleration diesel drive-by without mannequin 6. 


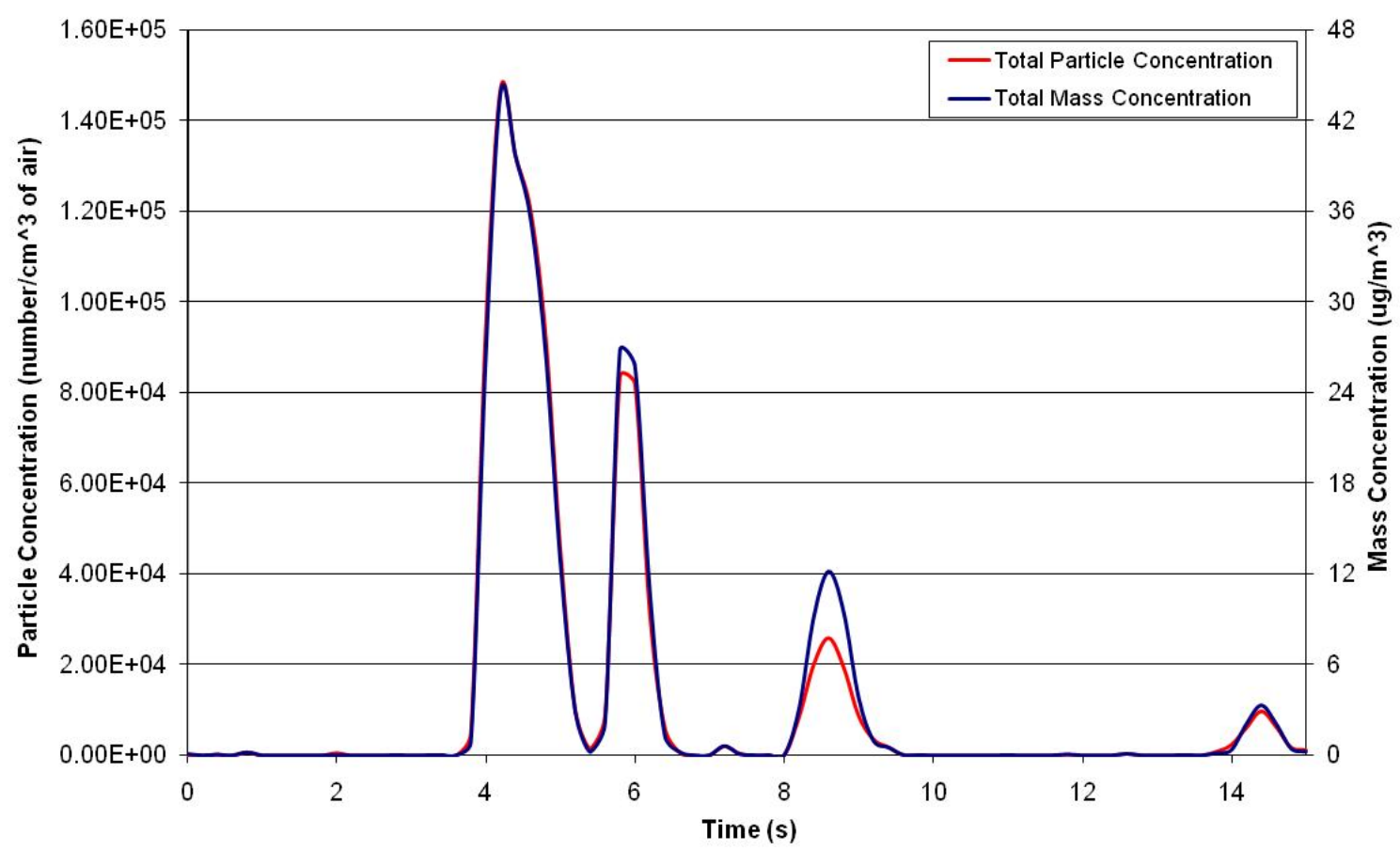

Figure 9-25: Medium acceleration diesel drive-by without mannequin 1.

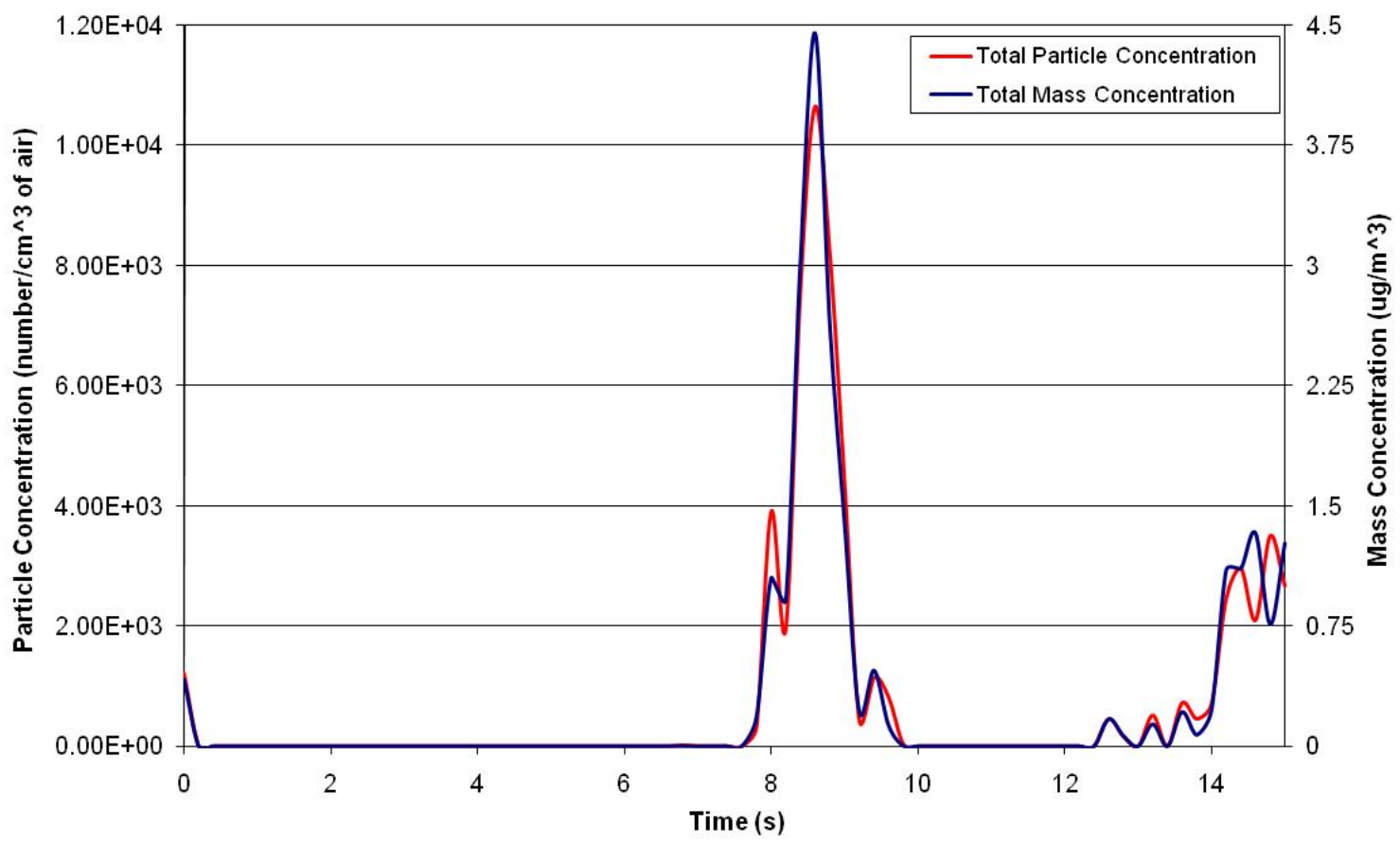

Figure 9-26: Medium acceleration diesel drive-by without mannequin 2. 


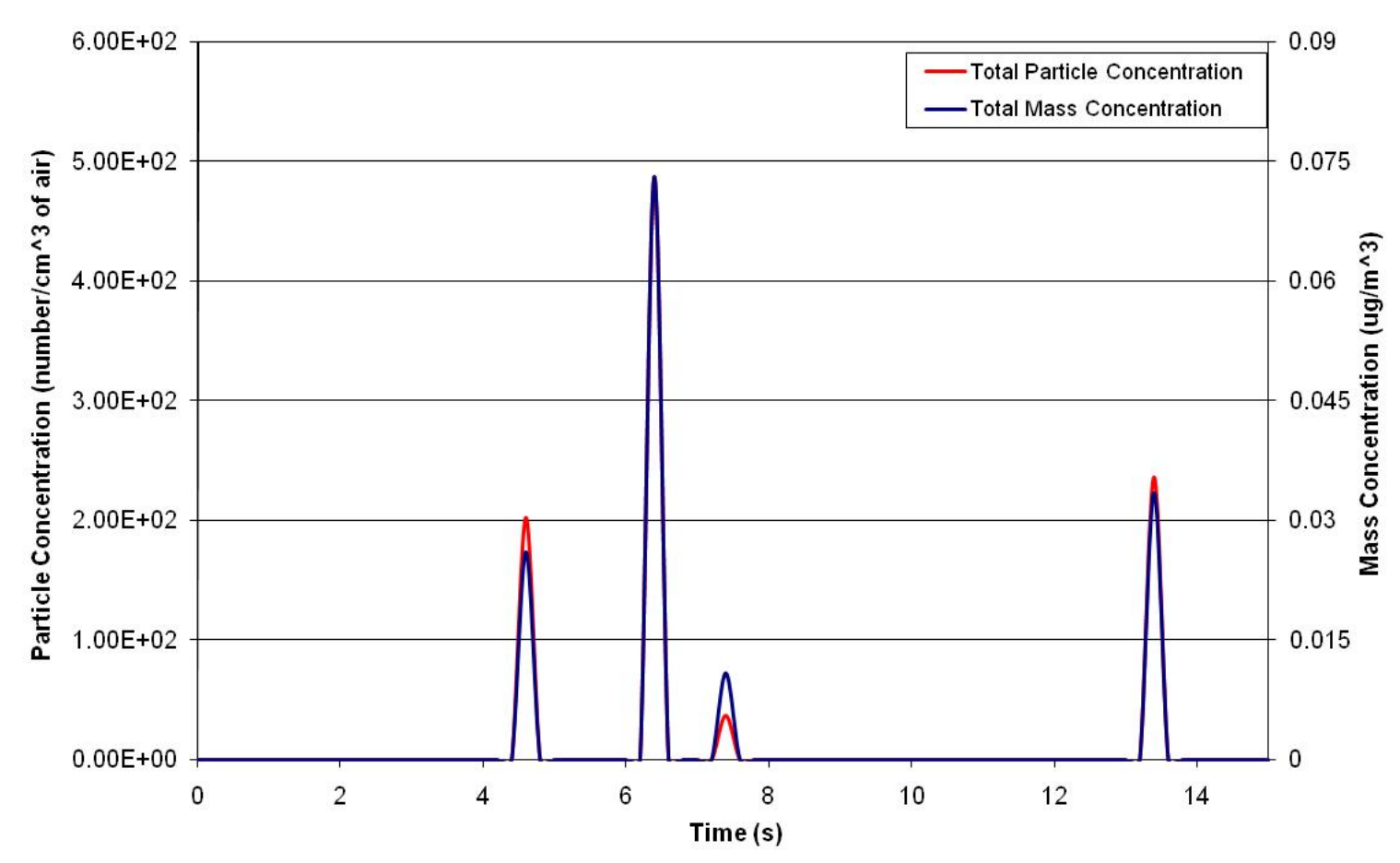

Figure 9-27: Medium acceleration diesel drive-by without mannequin 3.

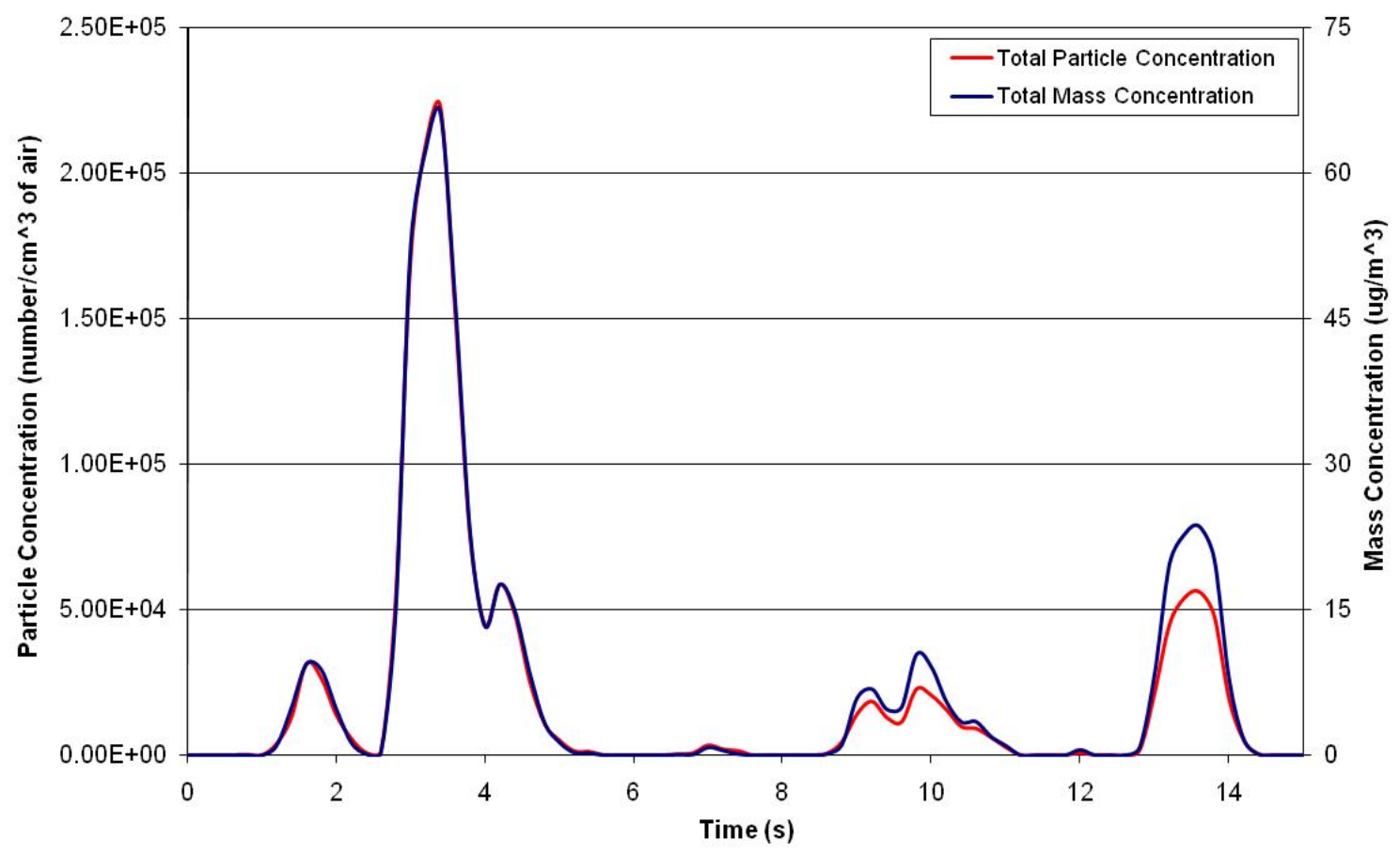

Figure 9-28: Medium acceleration diesel drive-by without mannequin 4. 


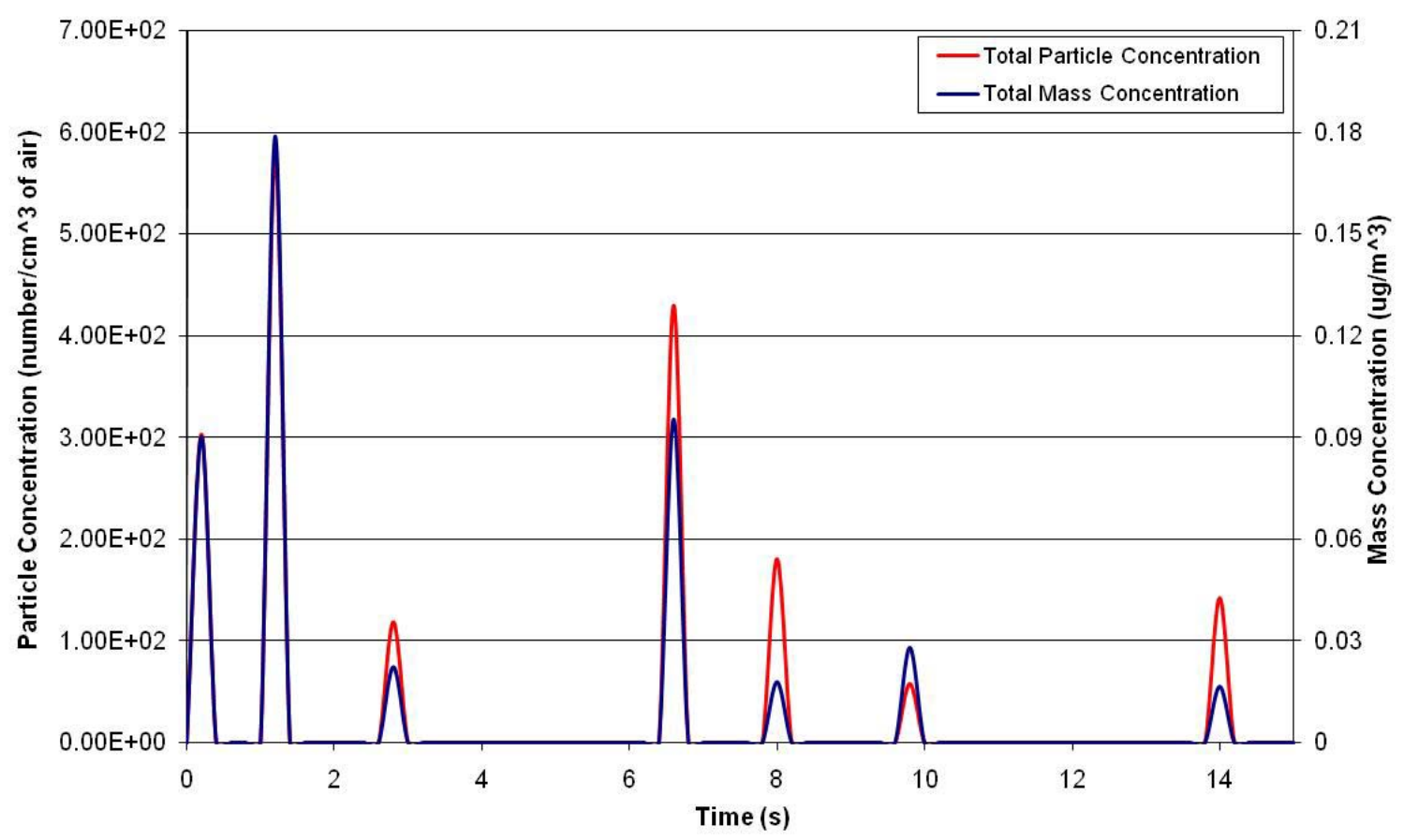

Figure 9-29: Medium acceleration diesel drive-by without mannequin 5.

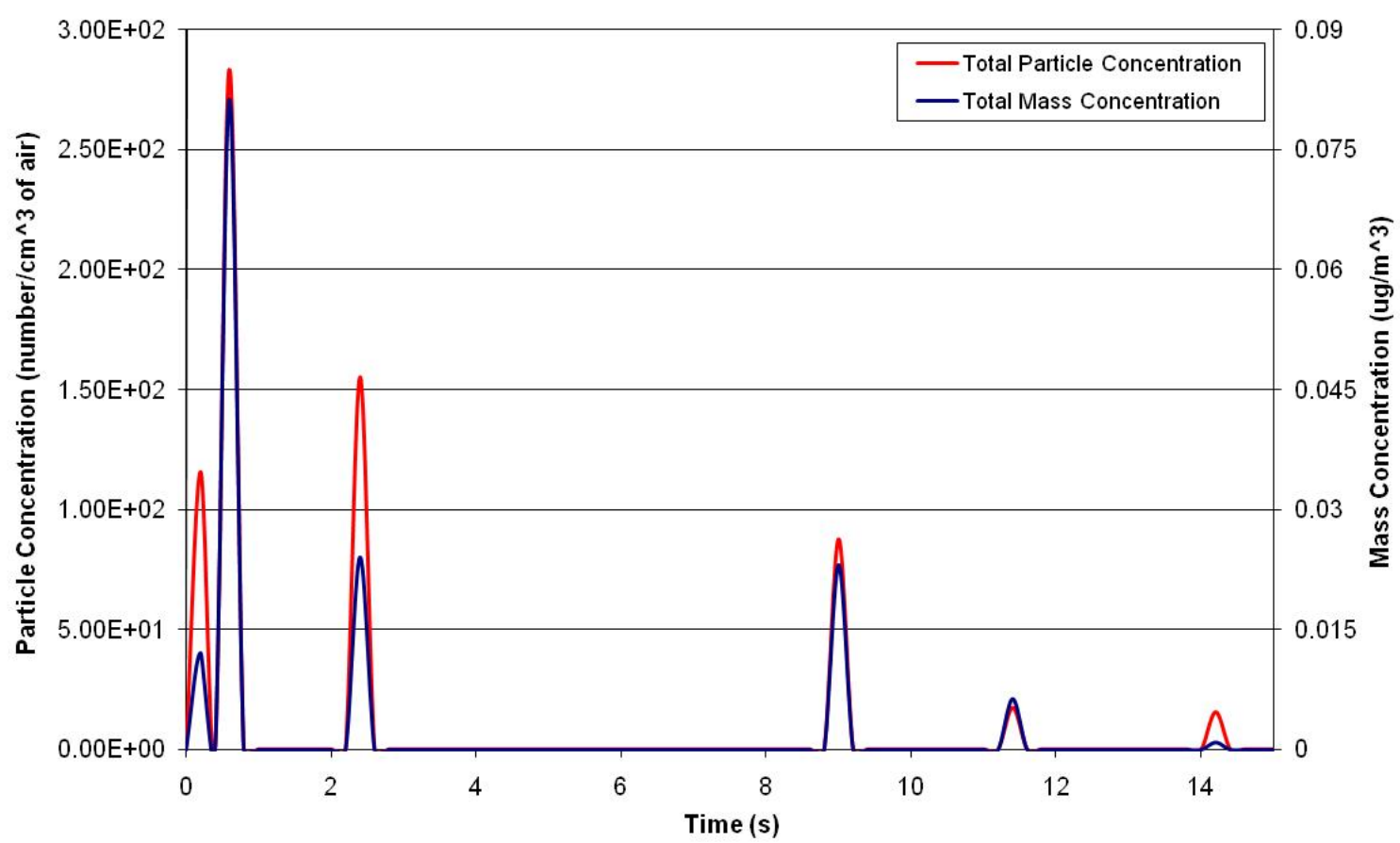

Figure 9-30: Medium acceleration diesel drive-by without mannequin 6. 


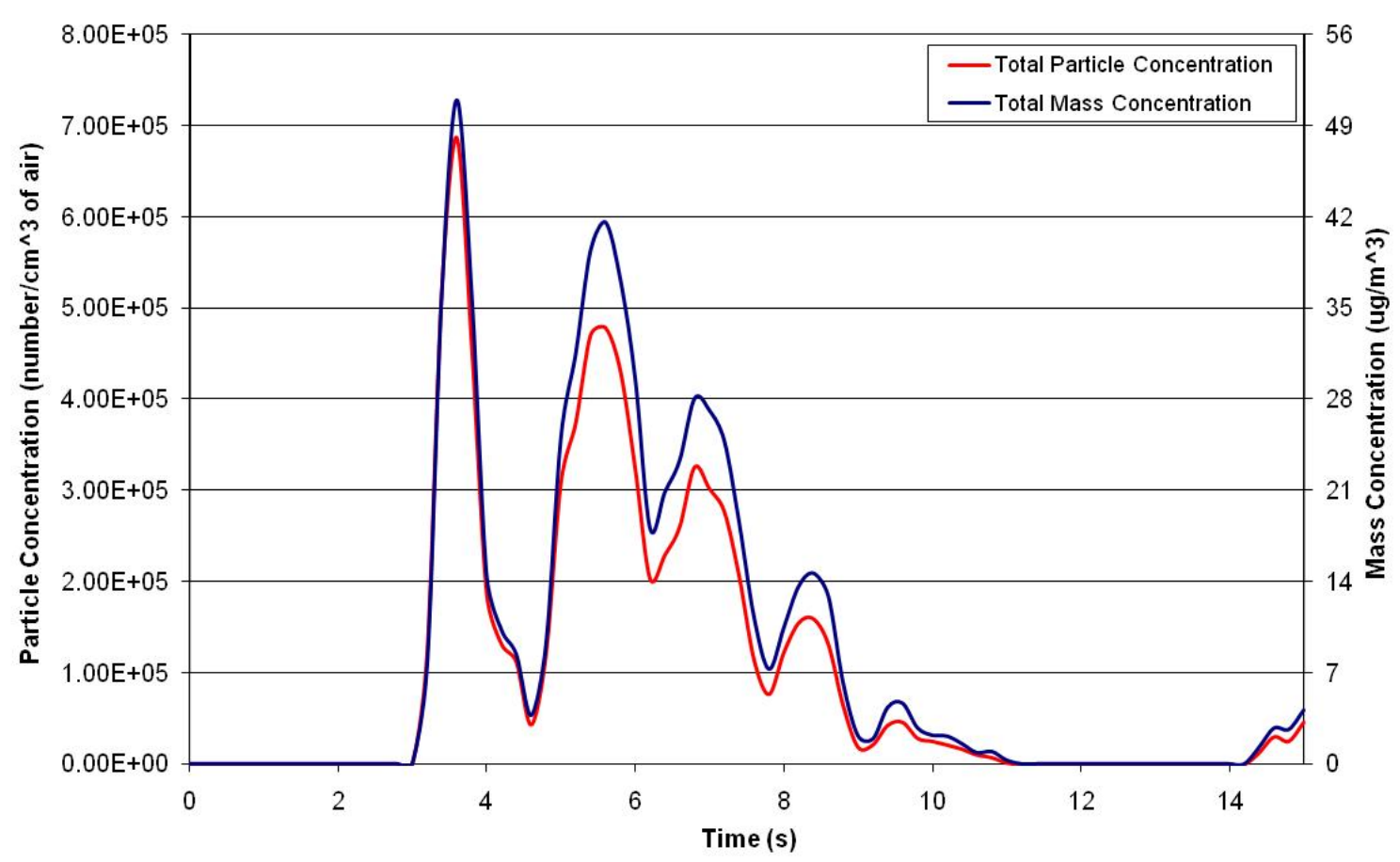

Figure 9-31: Cruising diesel drive-by without mannequin 1.

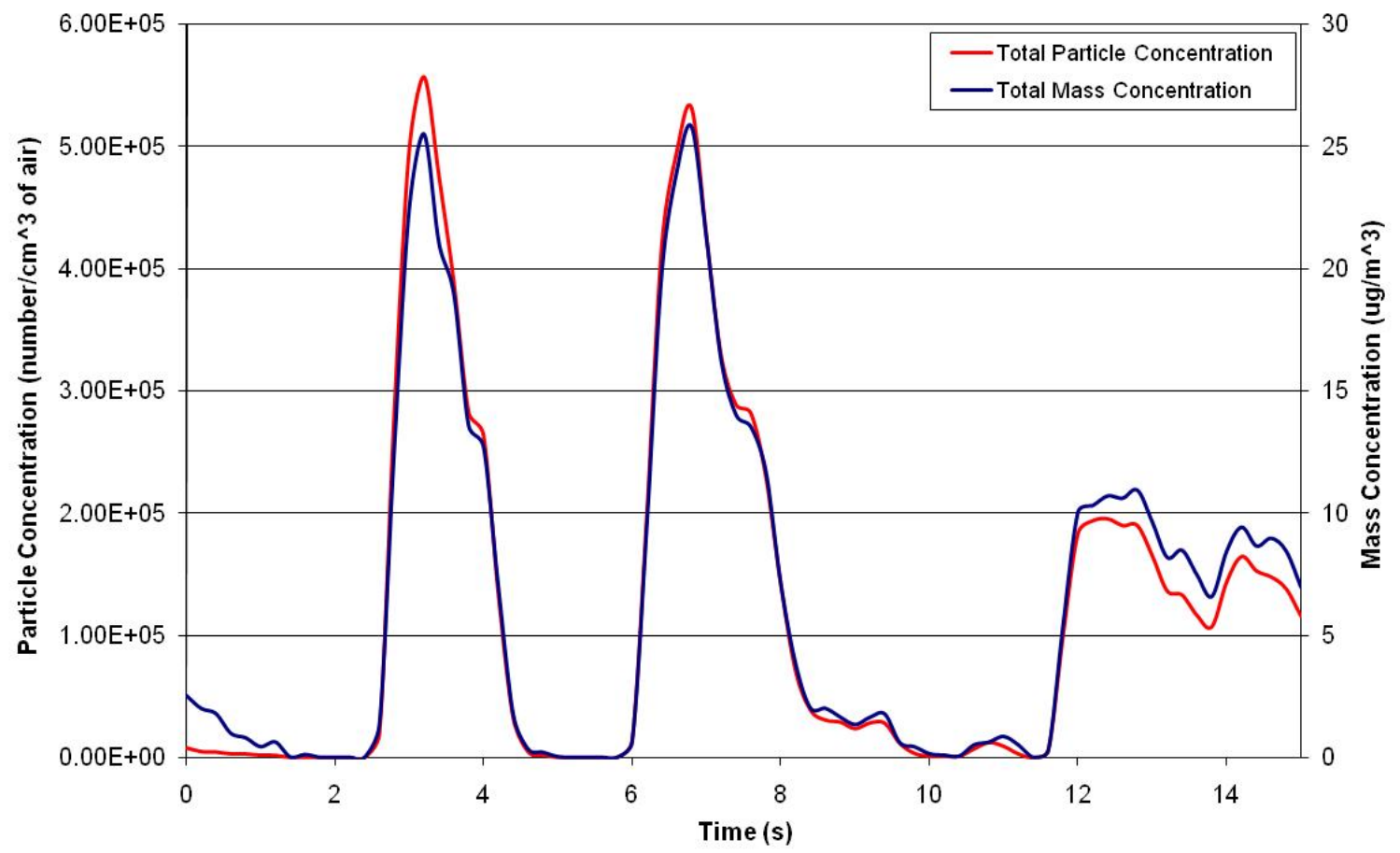

Figure 9-32: Cruising diesel drive-by without mannequin 2. 


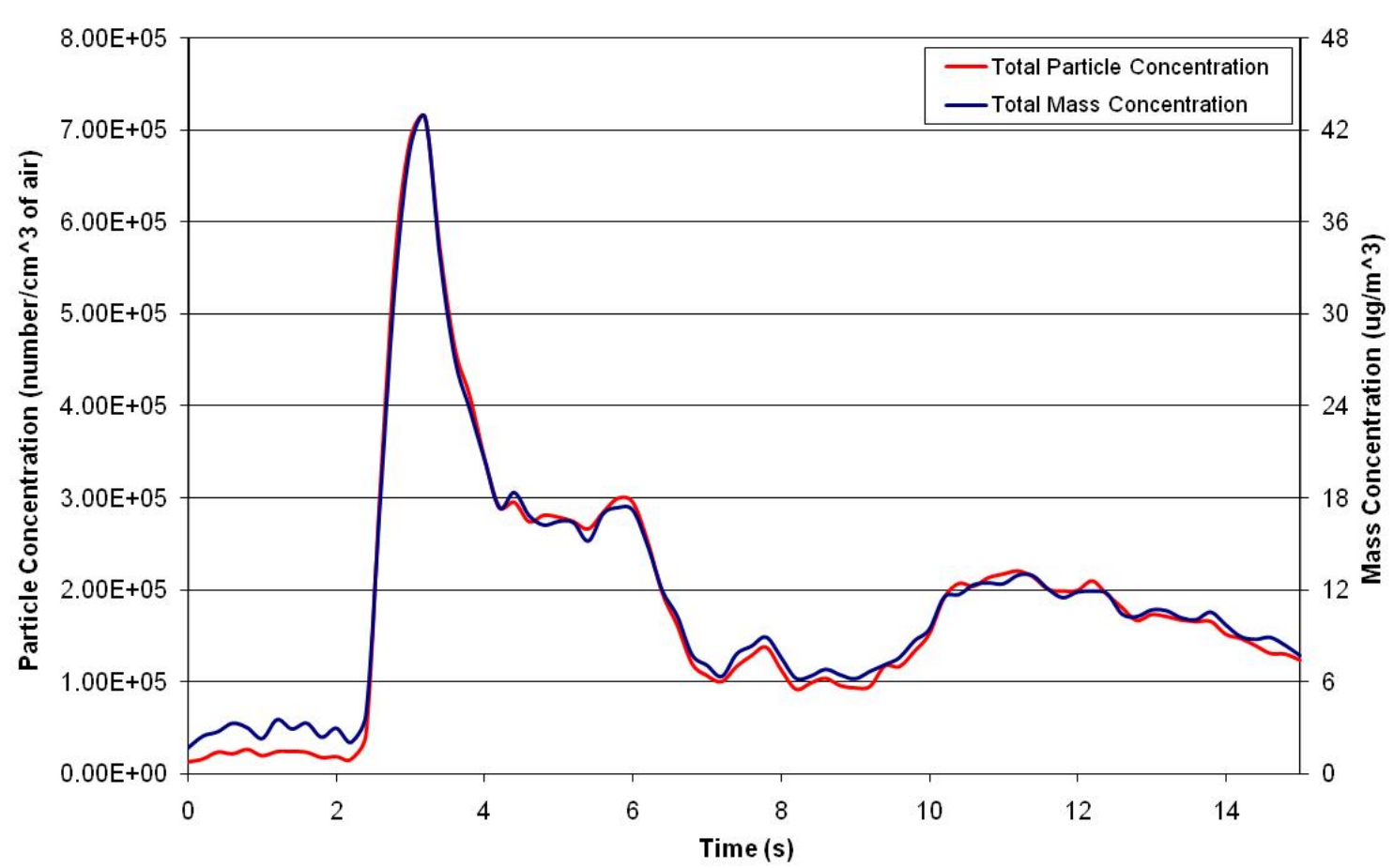

Figure 9-33: Cruising diesel drive-by without mannequin 3.

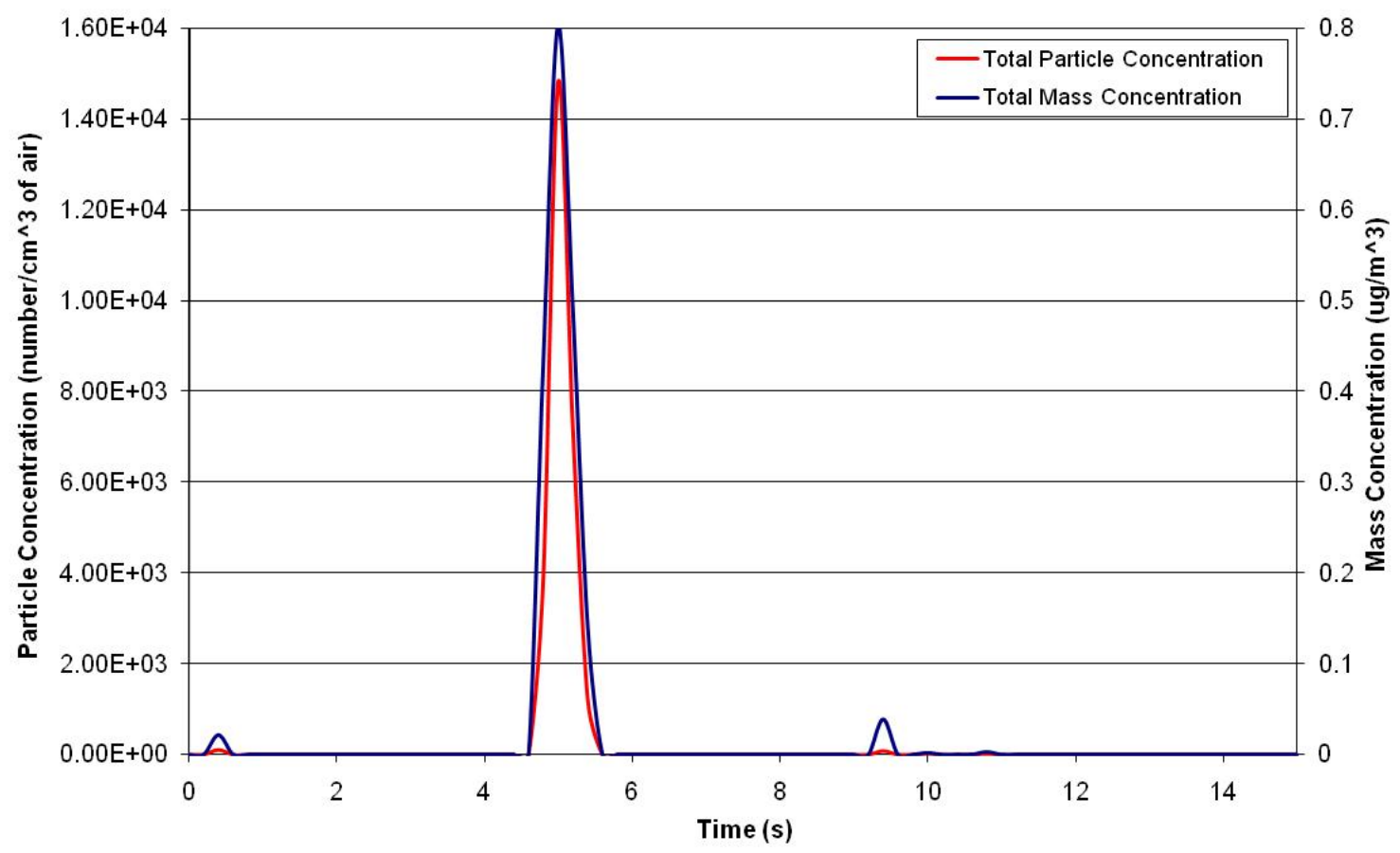

Figure 9-34: Cruising diesel drive-by without mannequin 4. 


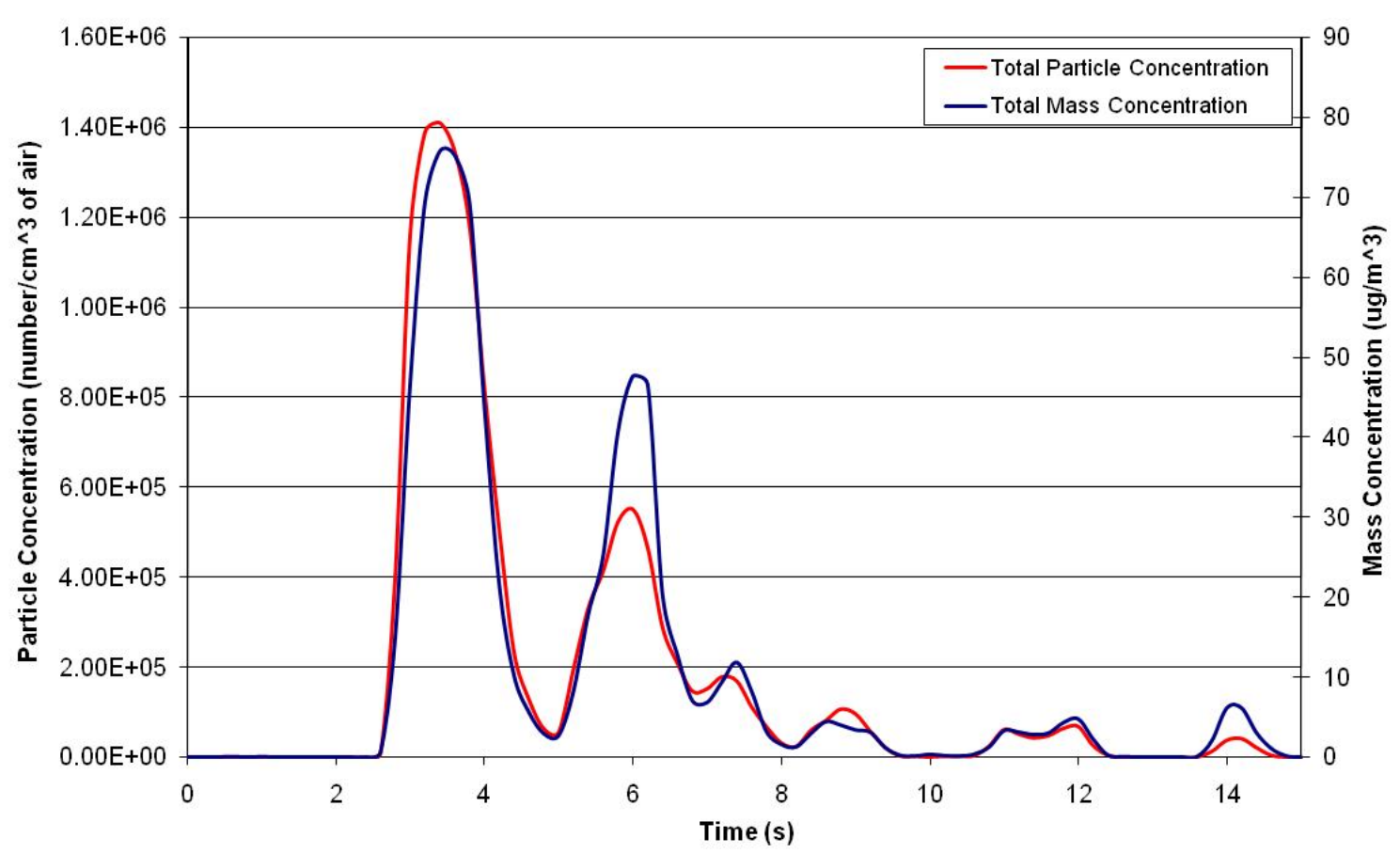

Figure 9-35: Cruising diesel drive-by without mannequin 5.

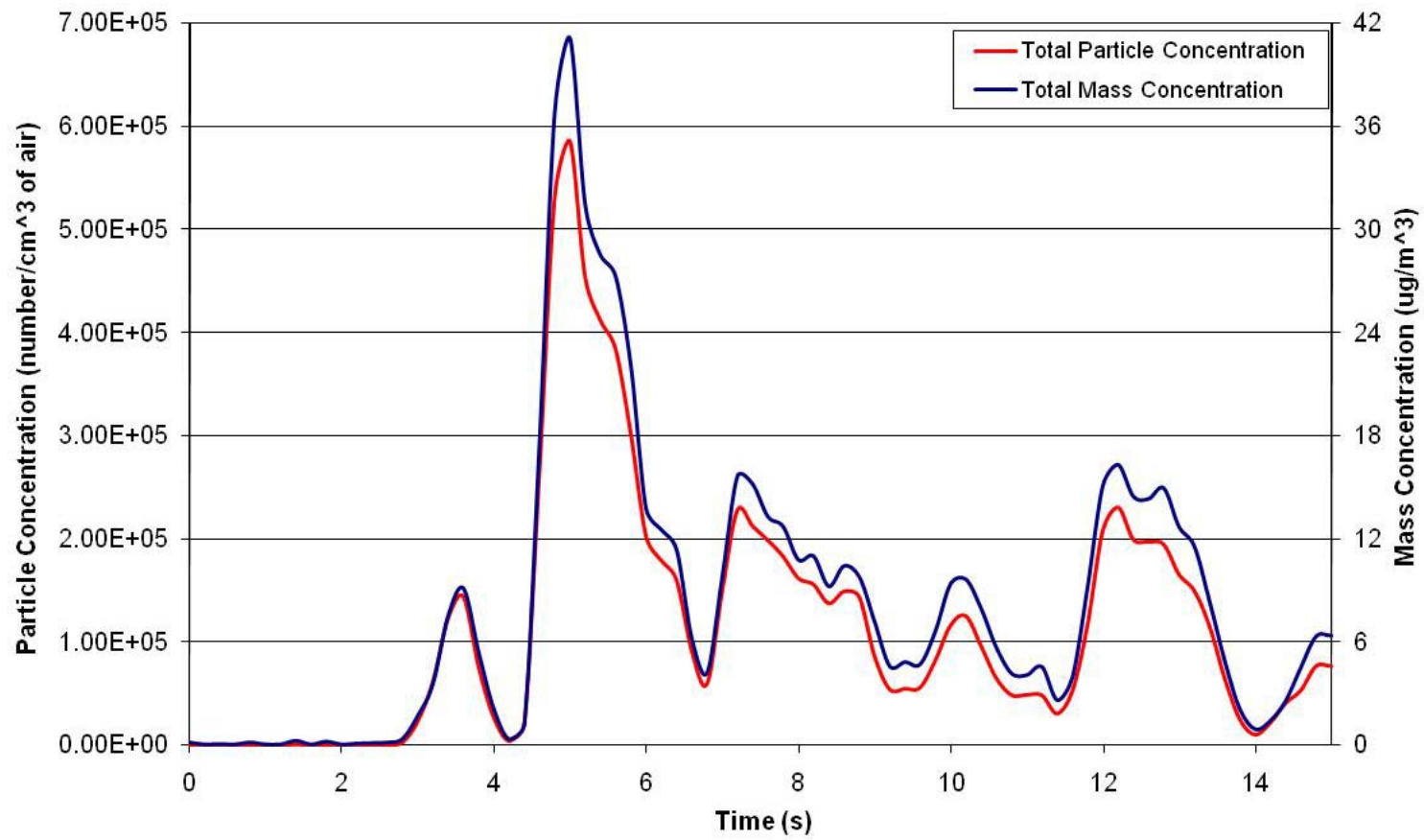

Figure 9-36: Cruising diesel drive-by without mannequin 6. 


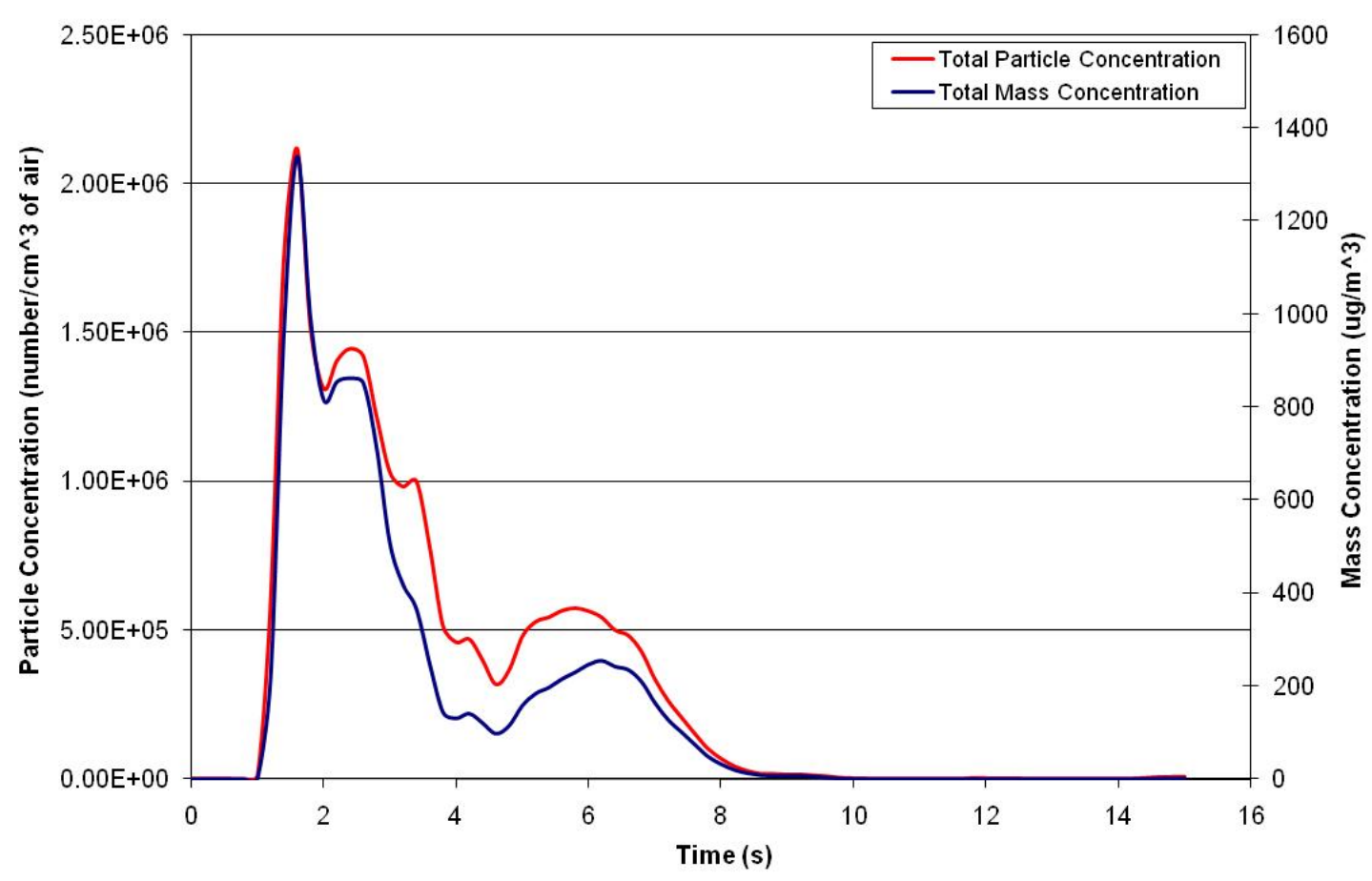

Figure 9-37: Hard acceleration diesel drive-by with baby 1.

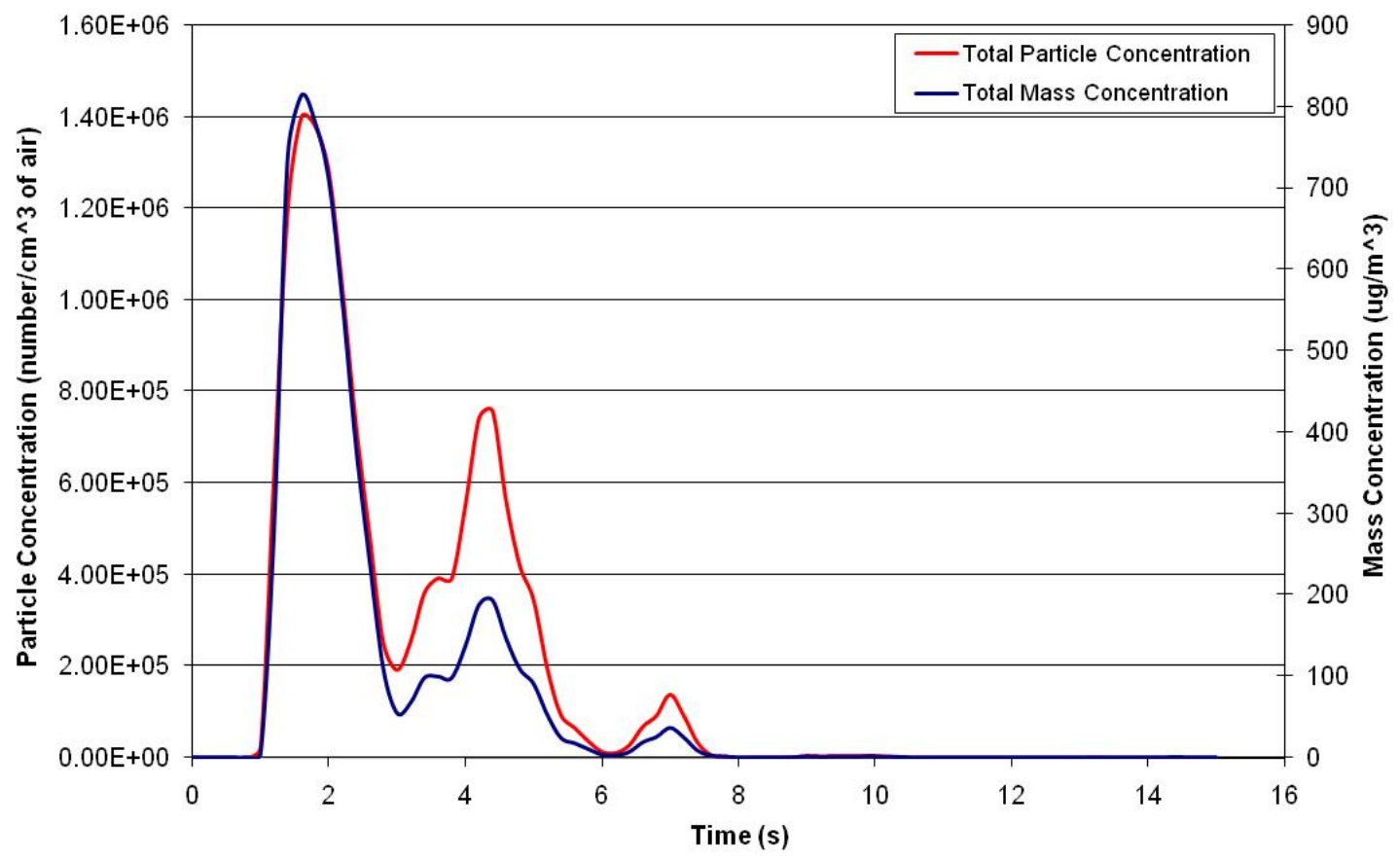

Figure 9-38: Hard acceleration diesel drive-by with baby 2. 


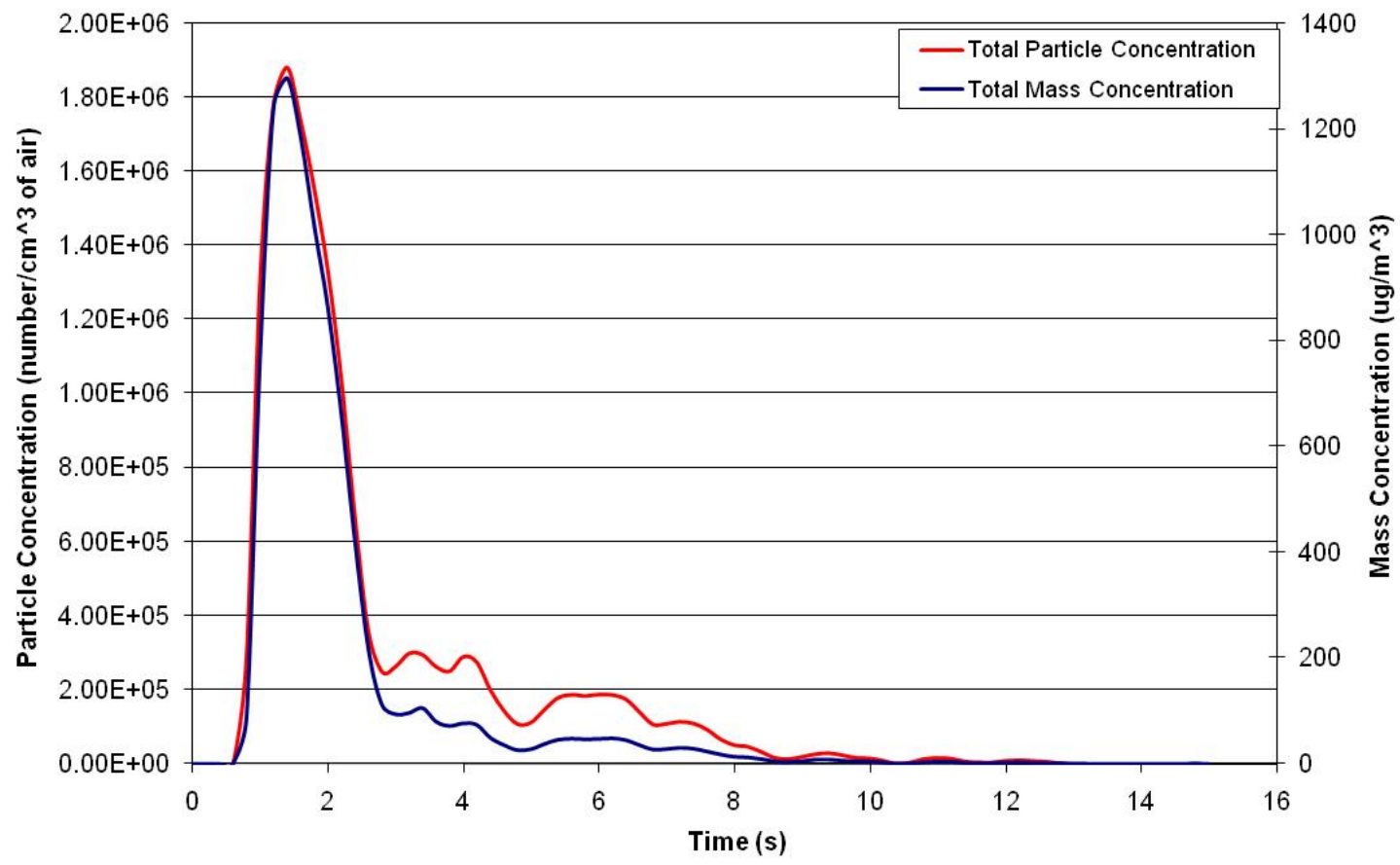

Figure 9-39: Hard acceleration diesel drive-by with baby 3 .

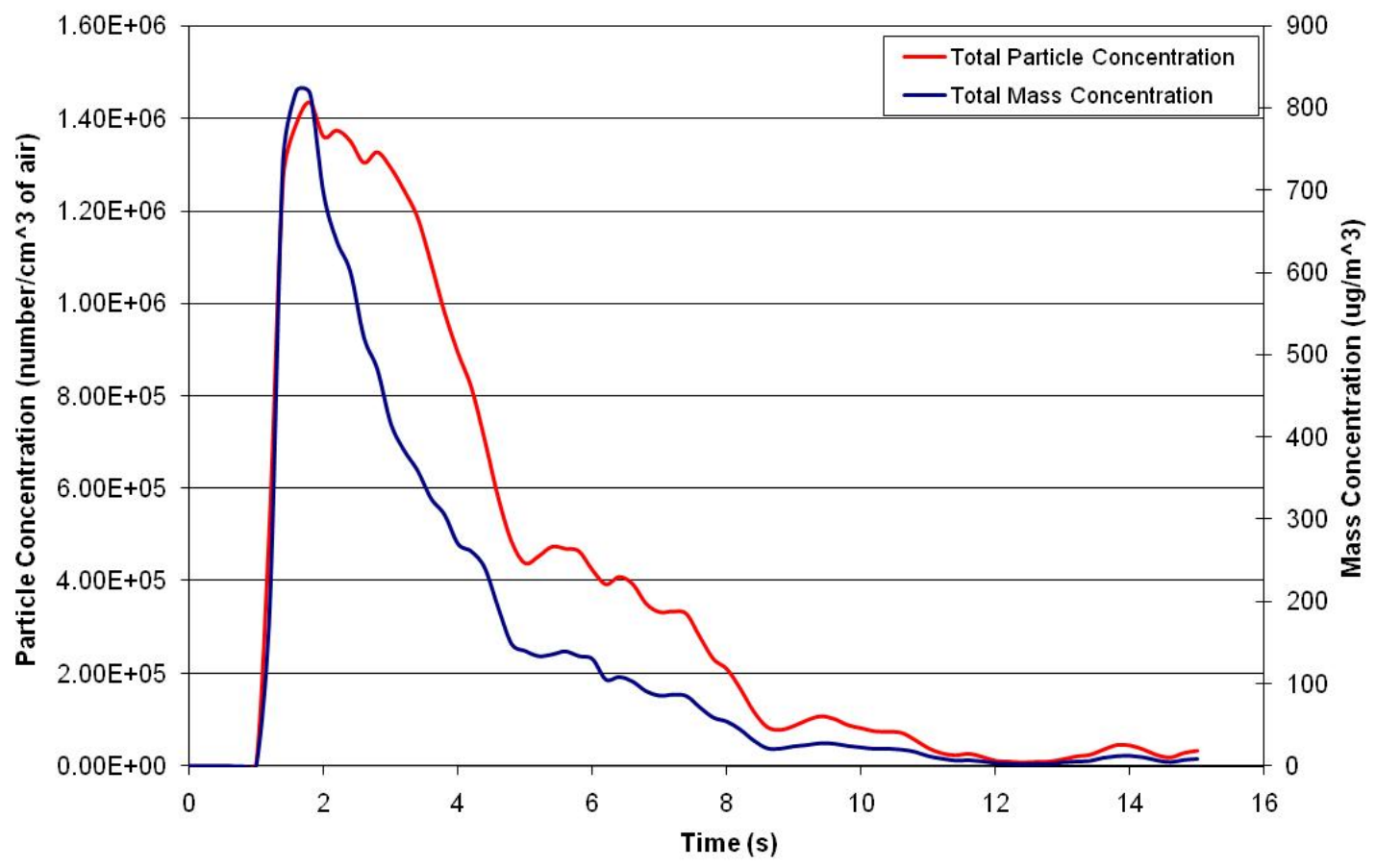

Figure 9-40: Hard acceleration diesel drive-by with baby 4 . 


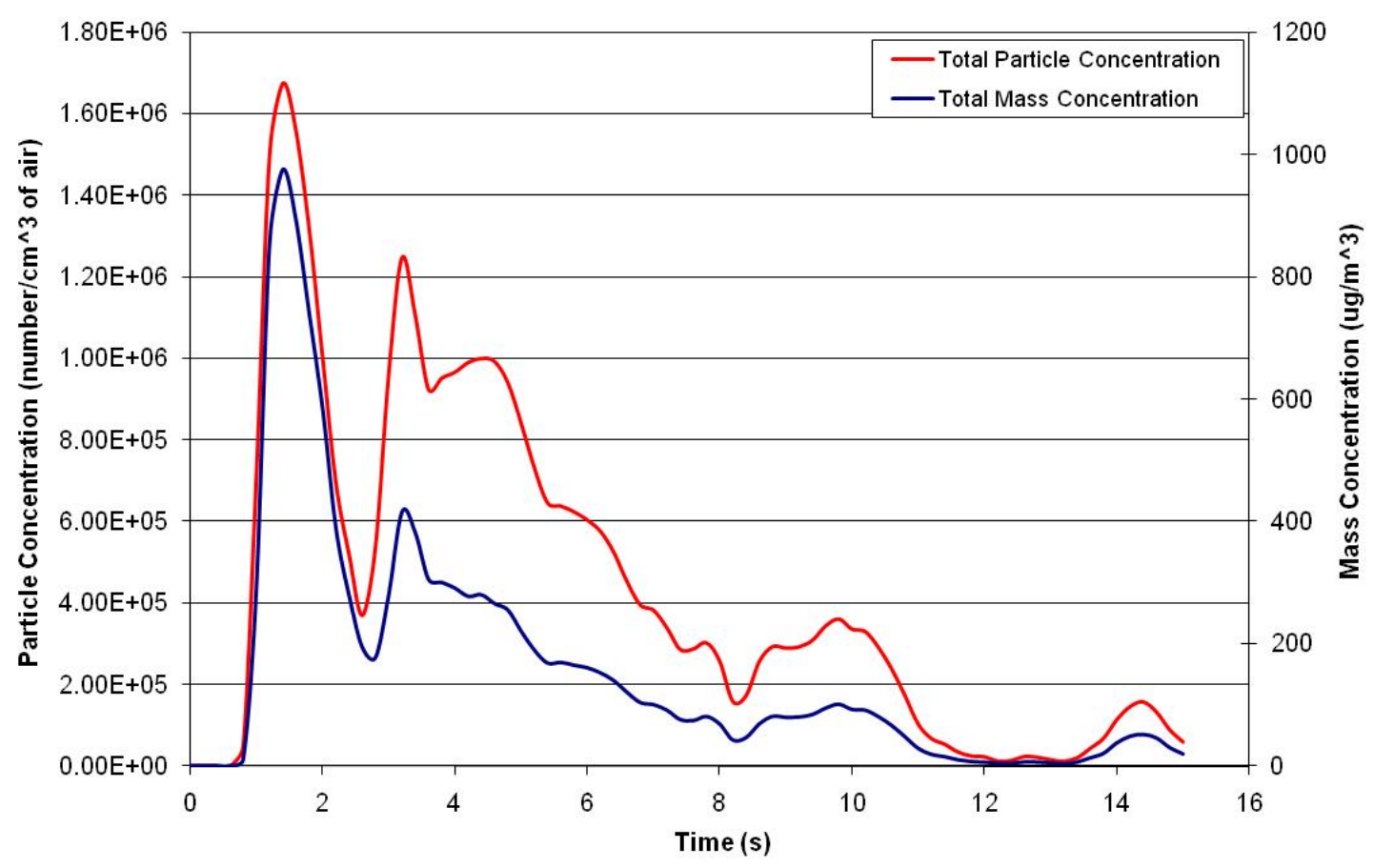

Figure 9-41: Hard acceleration diesel drive-by with baby 5 .

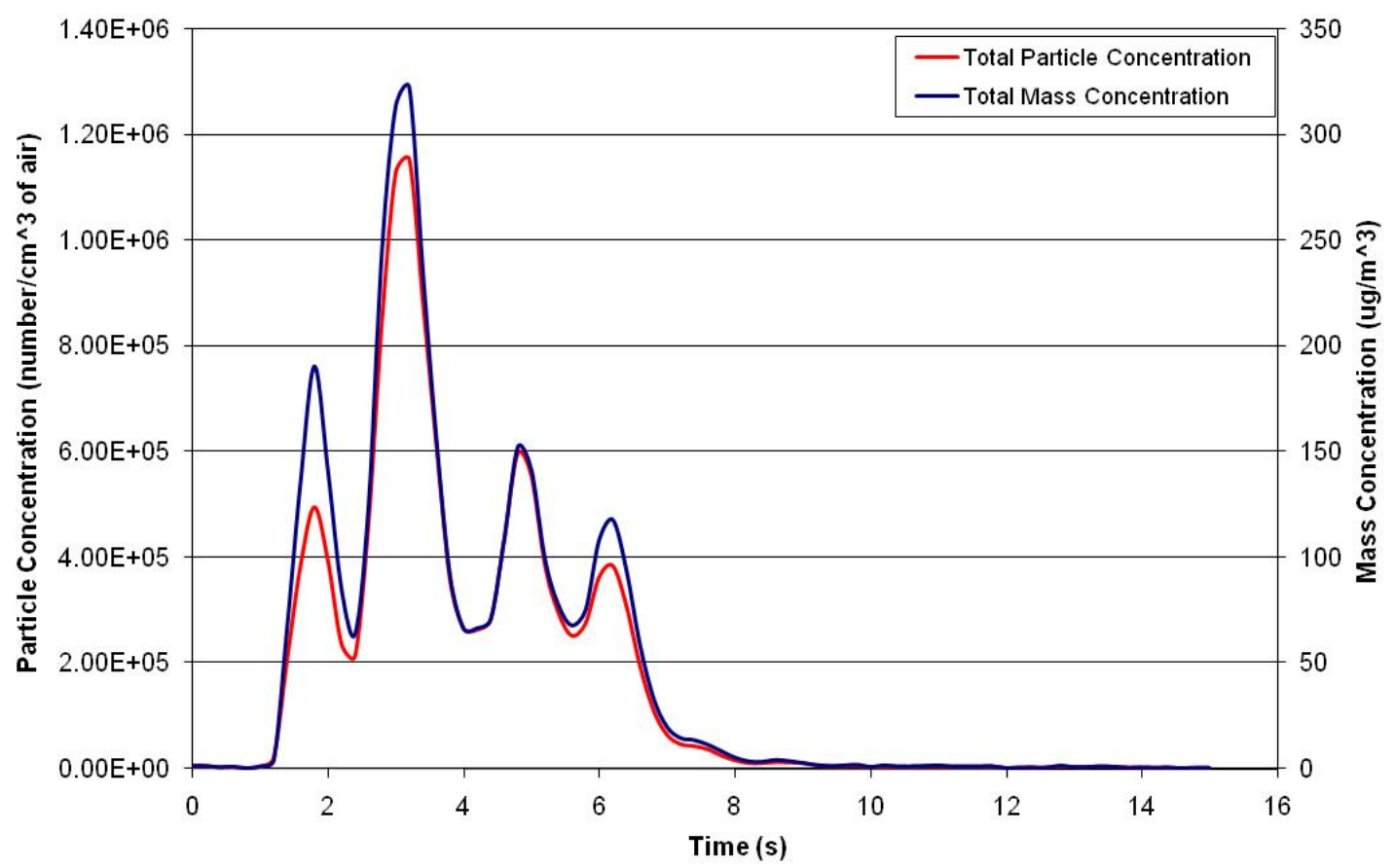

Figure 9-42: Hard acceleration diesel drive-by with baby 6 . 


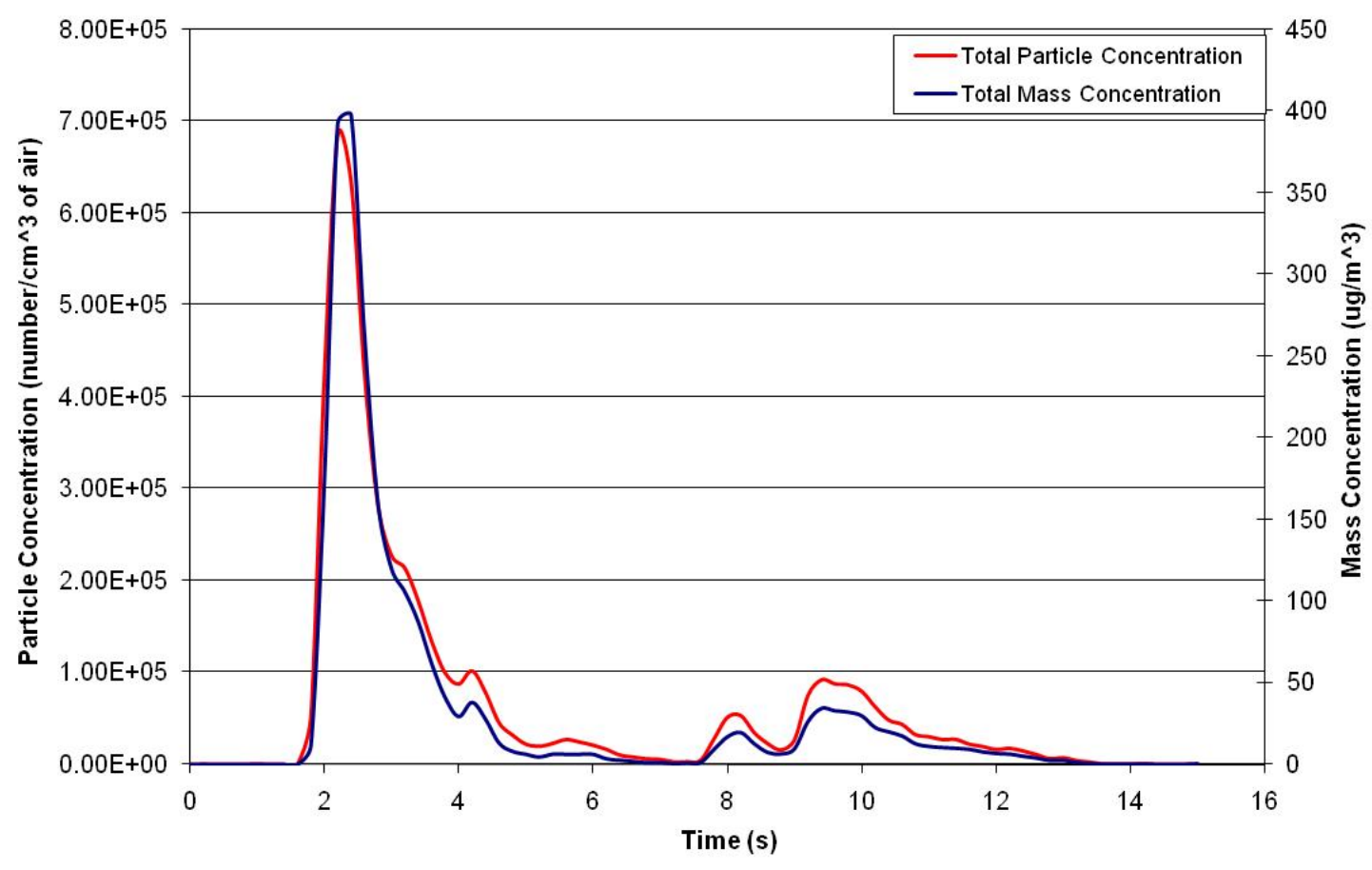

Figure 9-43: Medium acceleration diesel drive-by with baby 1.

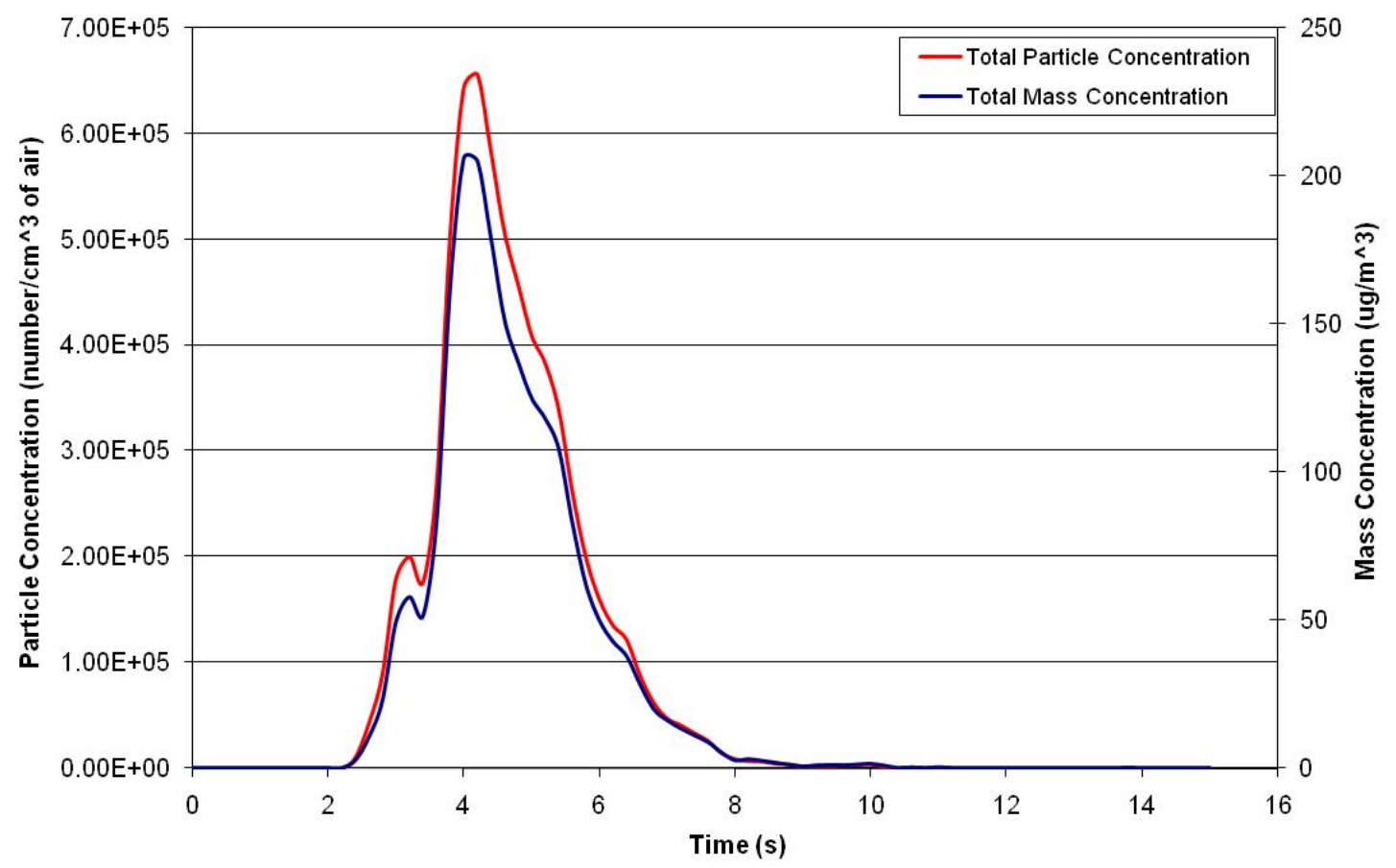

Figure 9-44: Medium acceleration diesel drive-by with baby 2. 


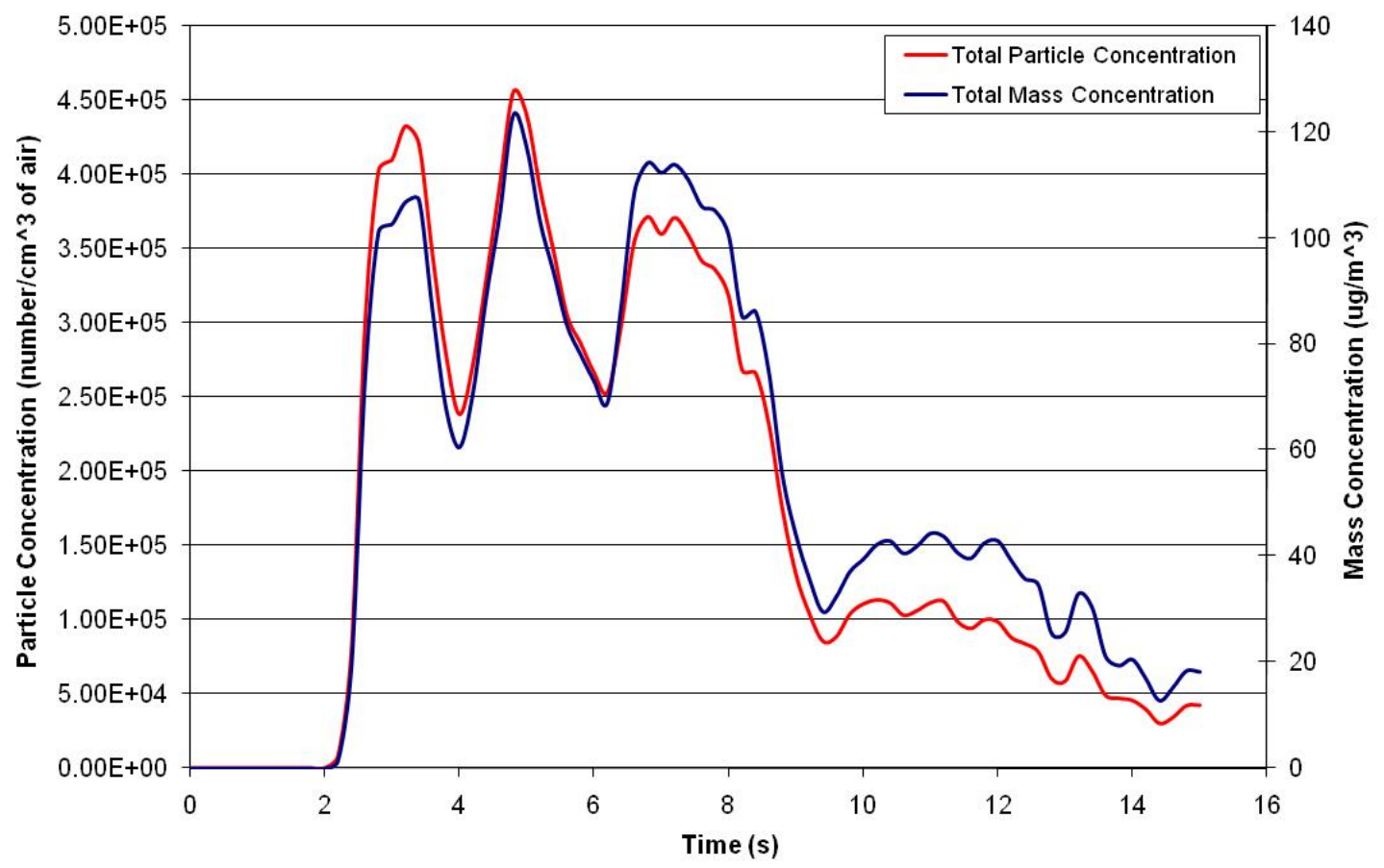

Figure 9-45: Medium acceleration diesel drive-by with baby 3 .

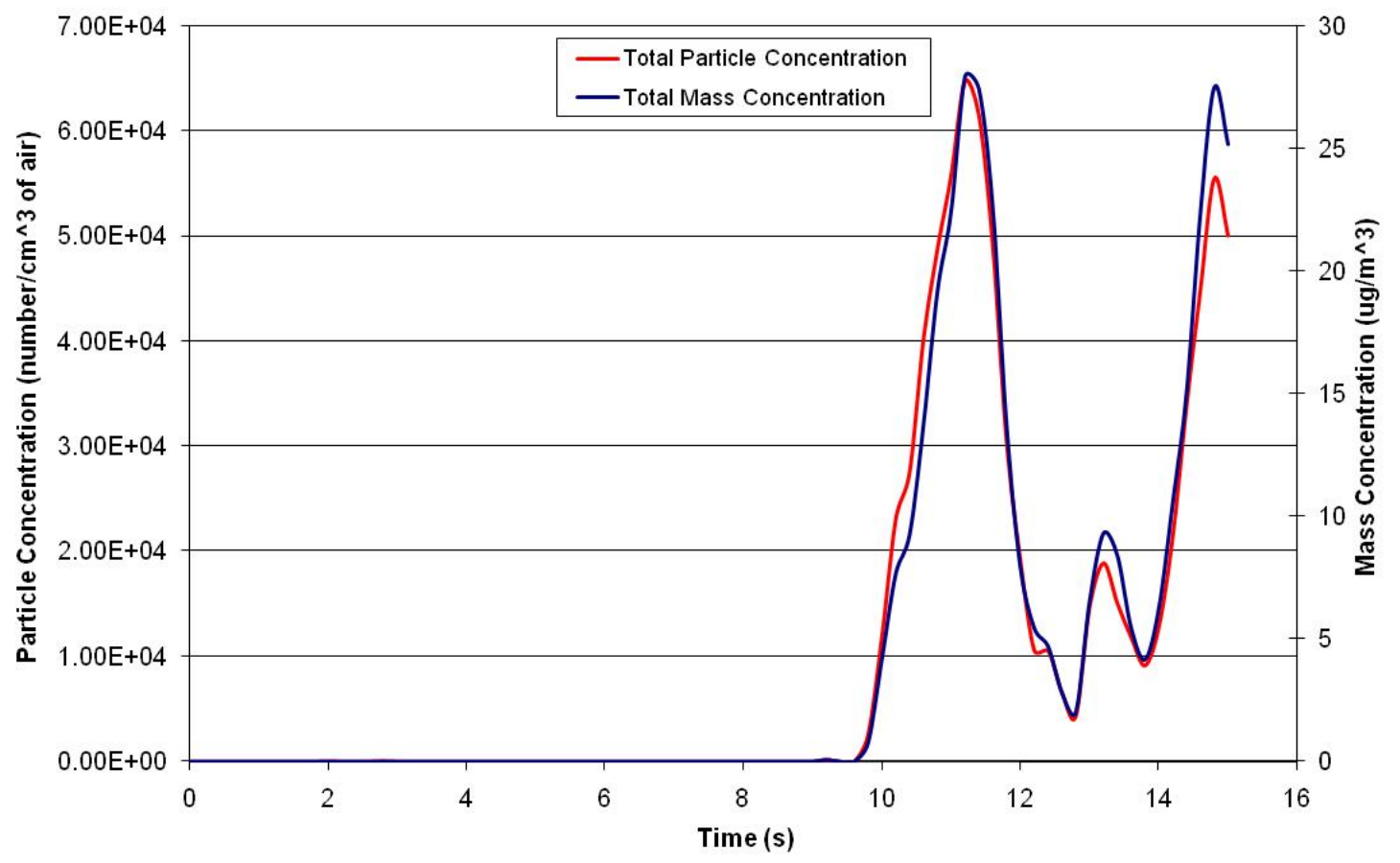

Figure 9-46: Medium acceleration diesel drive-by with baby 4 . 


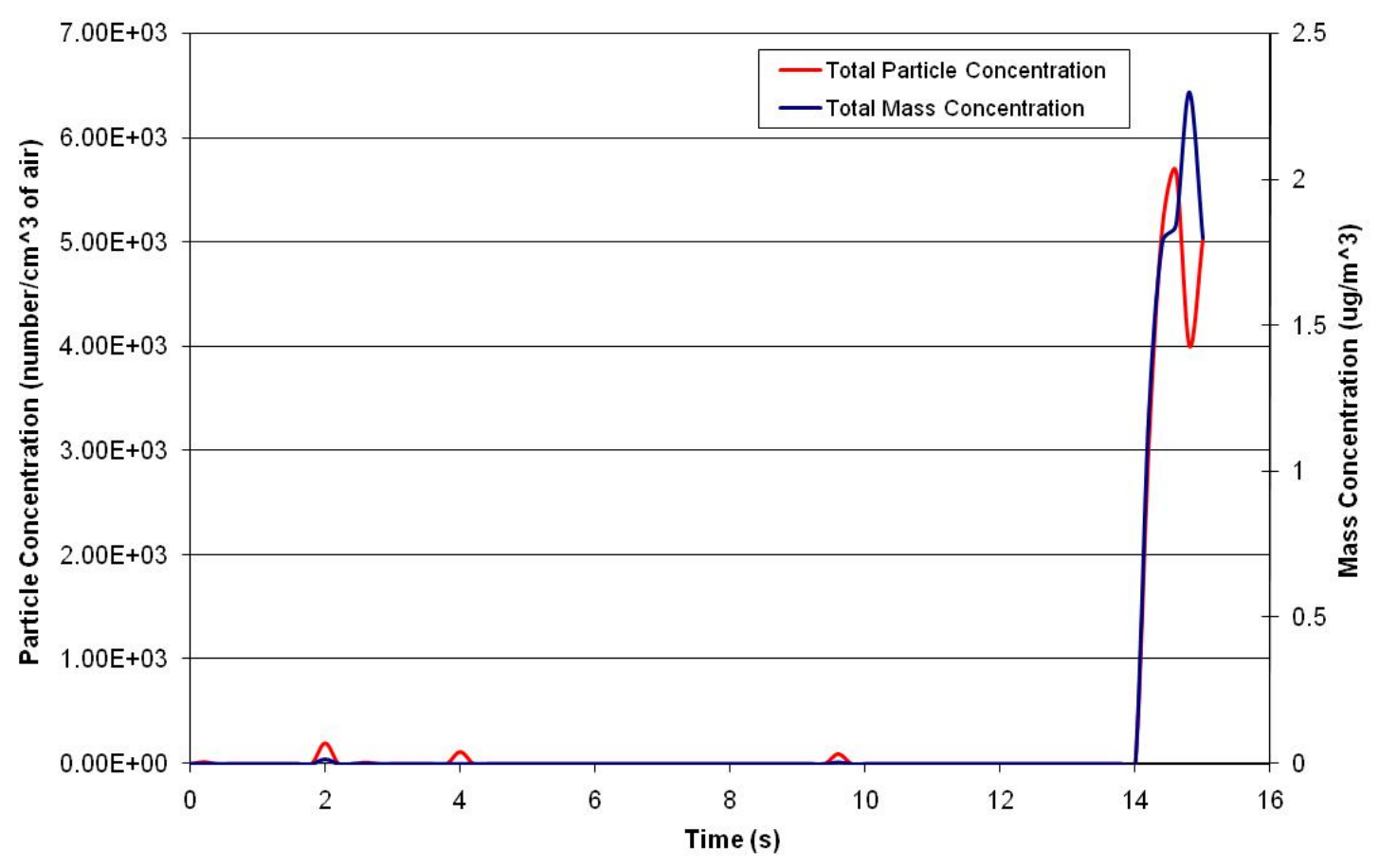

Figure 9-47: Medium acceleration diesel drive-by with baby 5 .

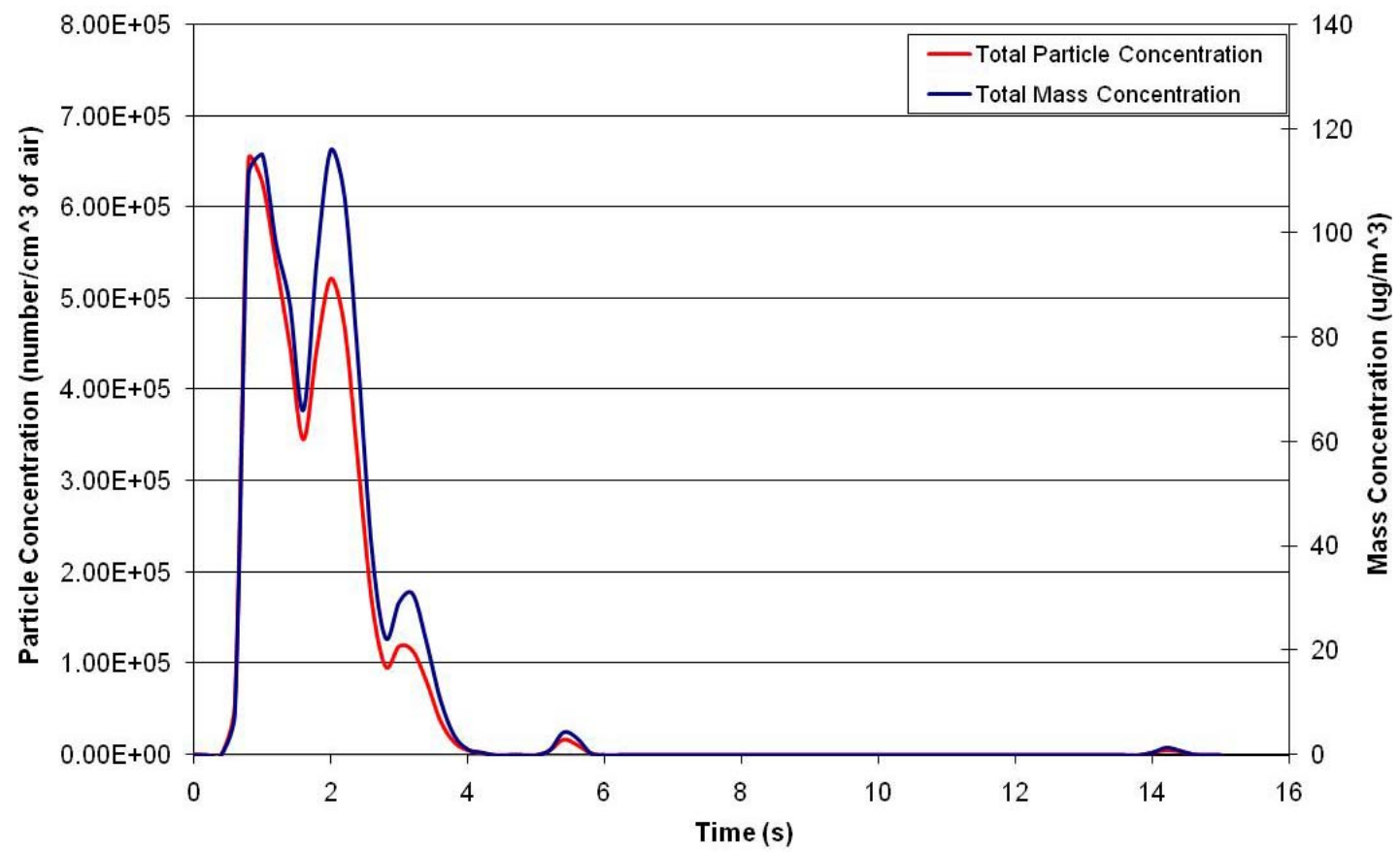

Figure 9-48: Medium acceleration diesel drive-by with baby 6 . 


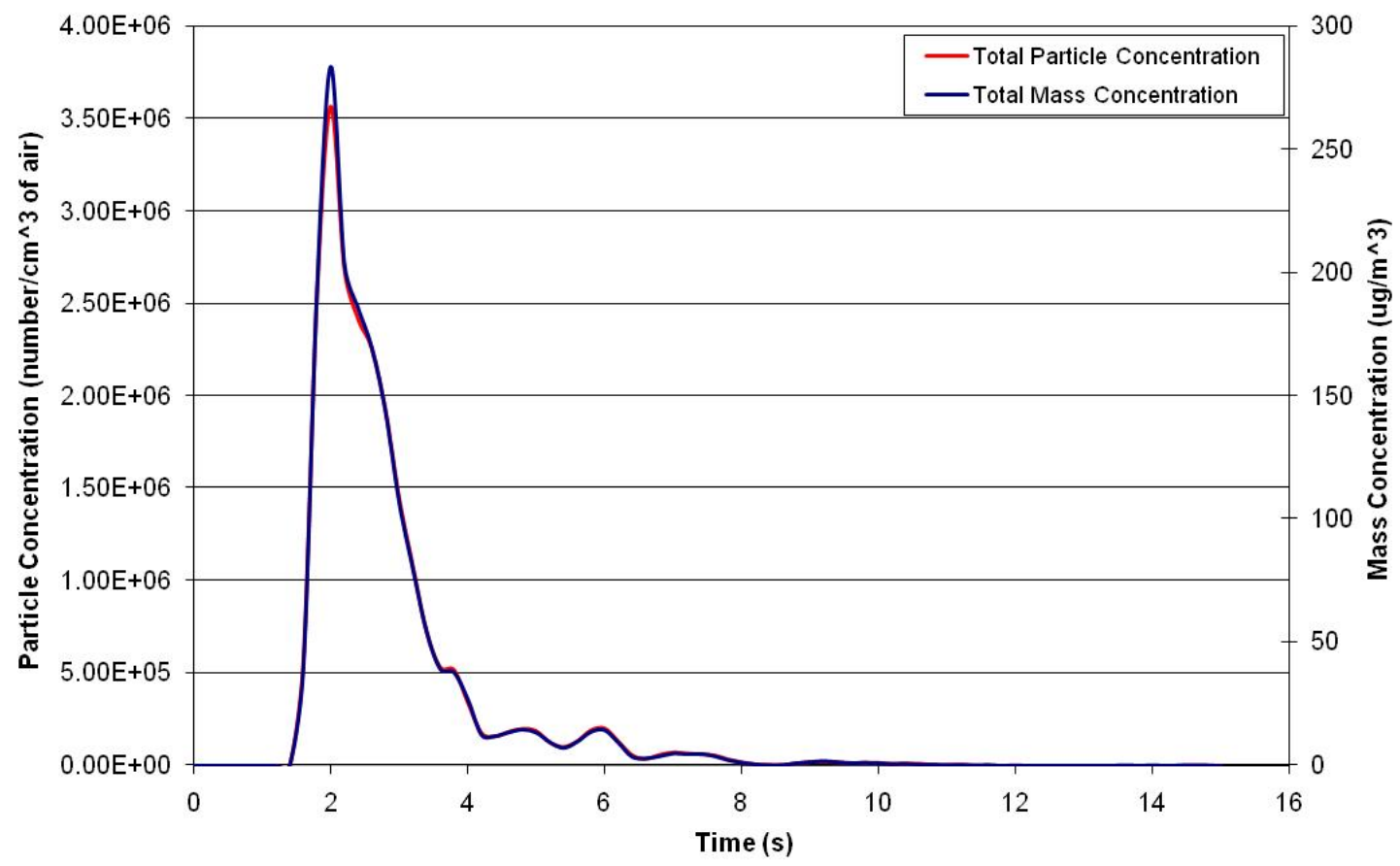

Figure 9-49: Cruising diesel drive-by with baby 1 .

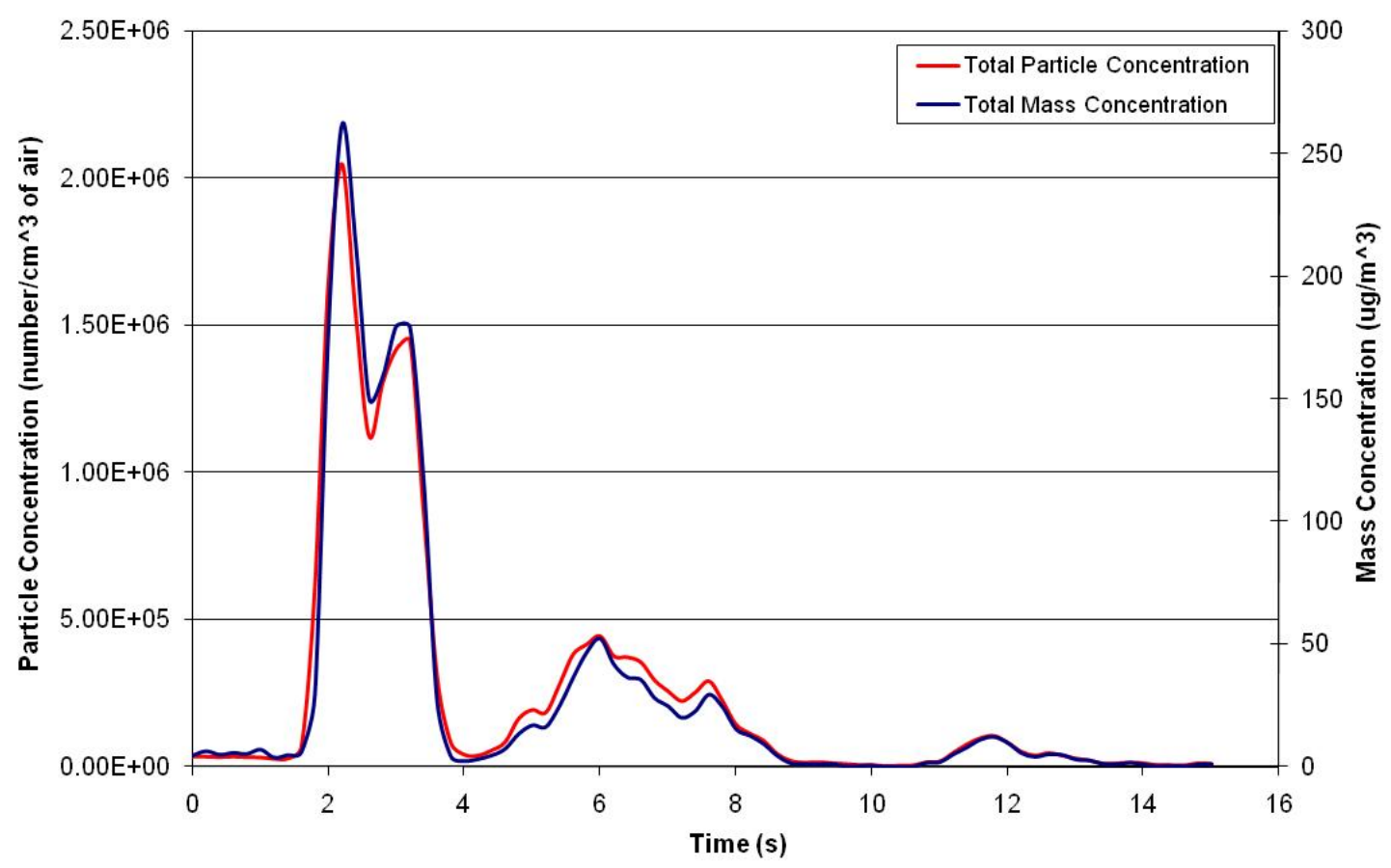

Figure 9-50: Cruising diesel drive-by with baby 2 . 


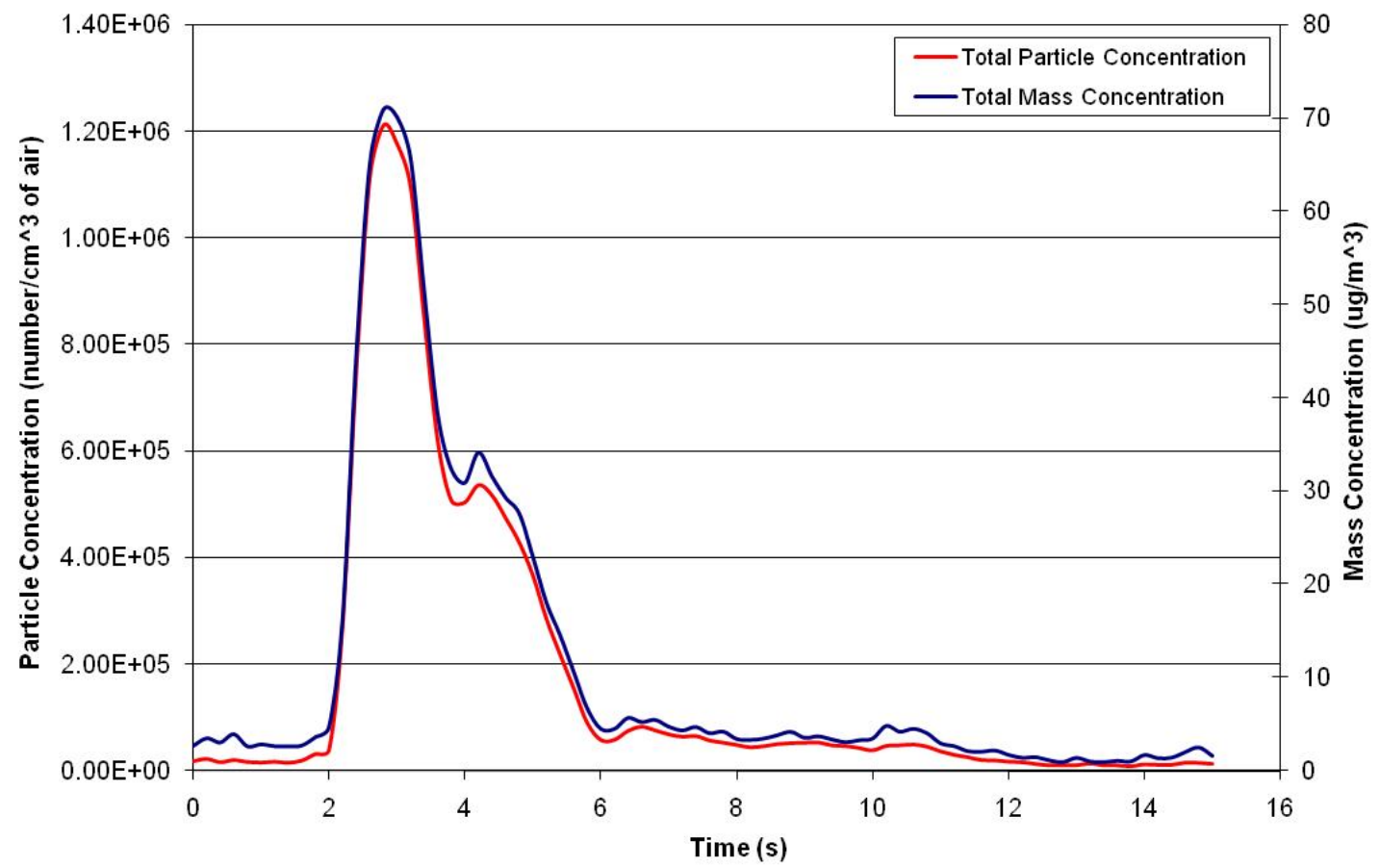

Figure 9-51: Cruising diesel drive-by with baby 3 .

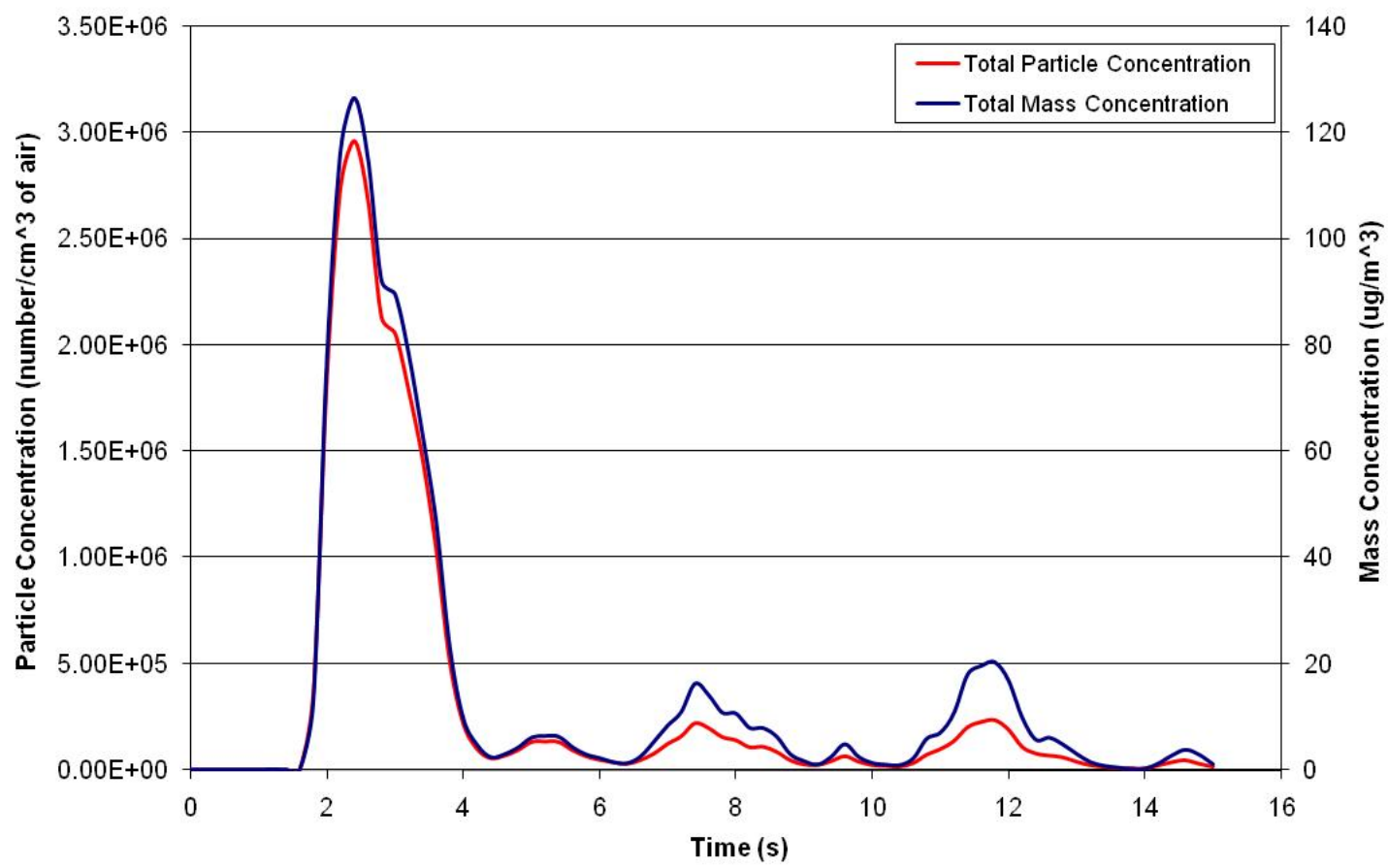

Figure 9-52: Cruising diesel drive-by with baby 4. 


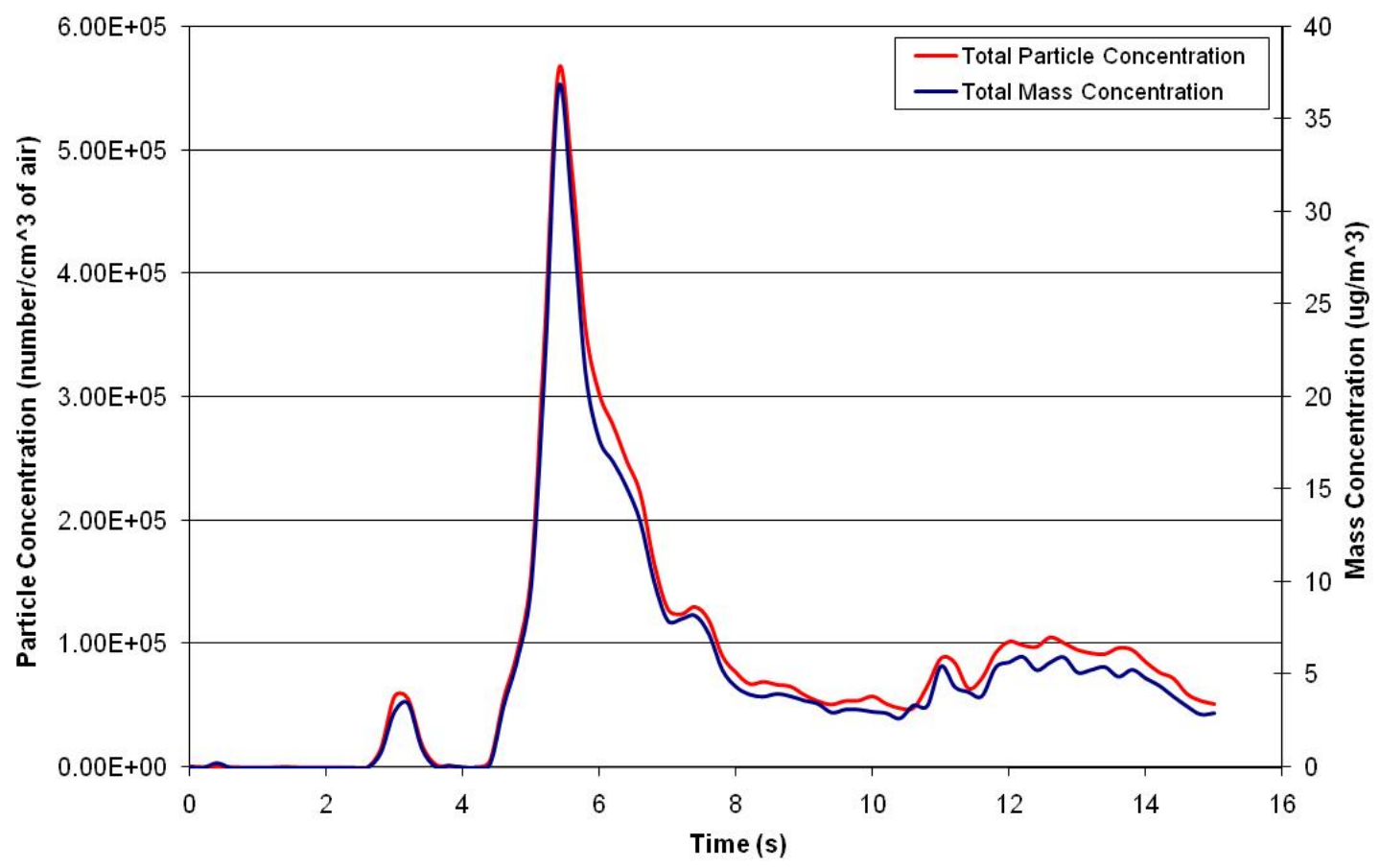

Figure 9-53: Cruising diesel drive-by with baby 5 .

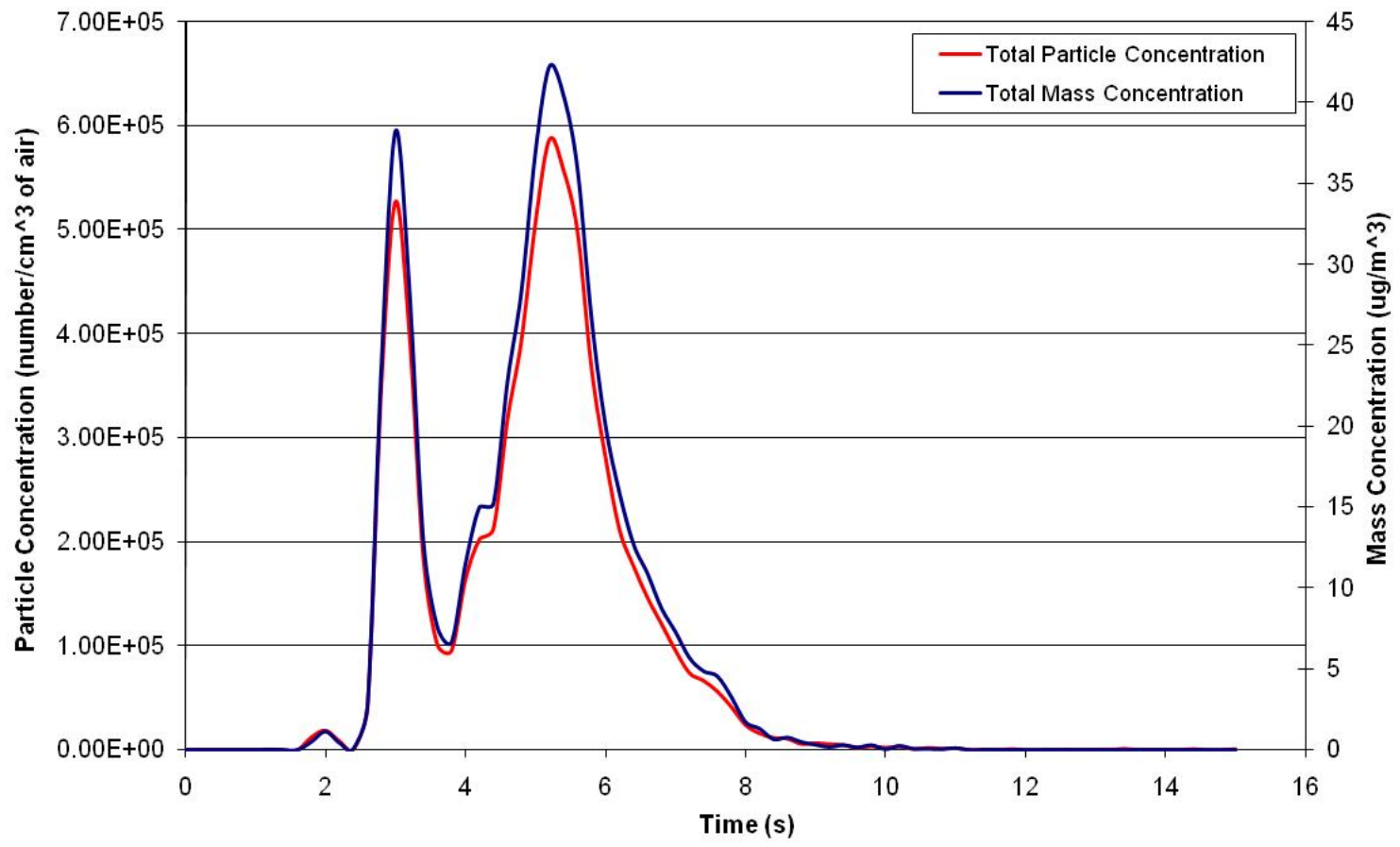

Figure 9-54: Cruising diesel drive-by with baby 6 . 


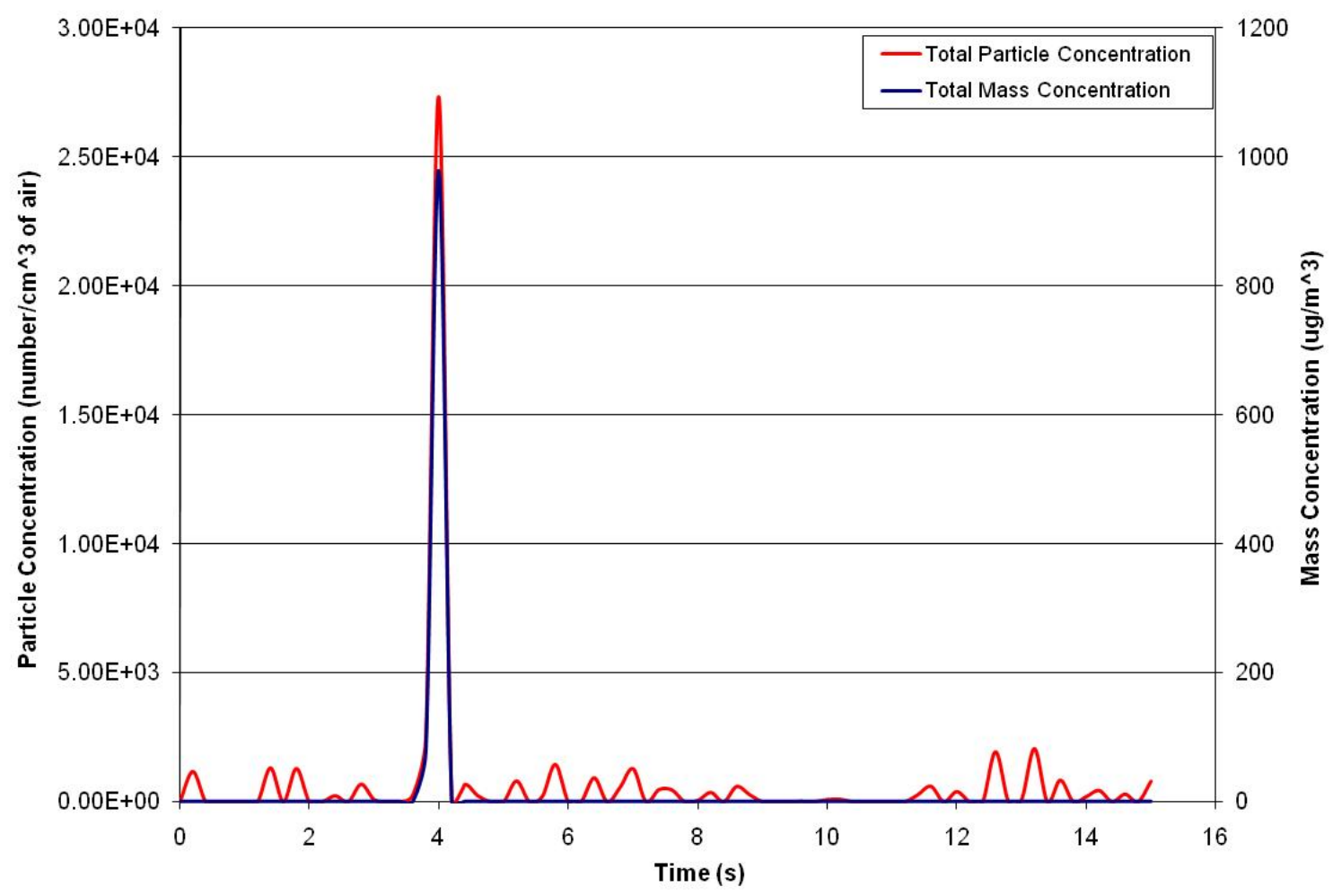

Figure 9-55: Hard acceleration gasoline drive-by with mannequin 1.

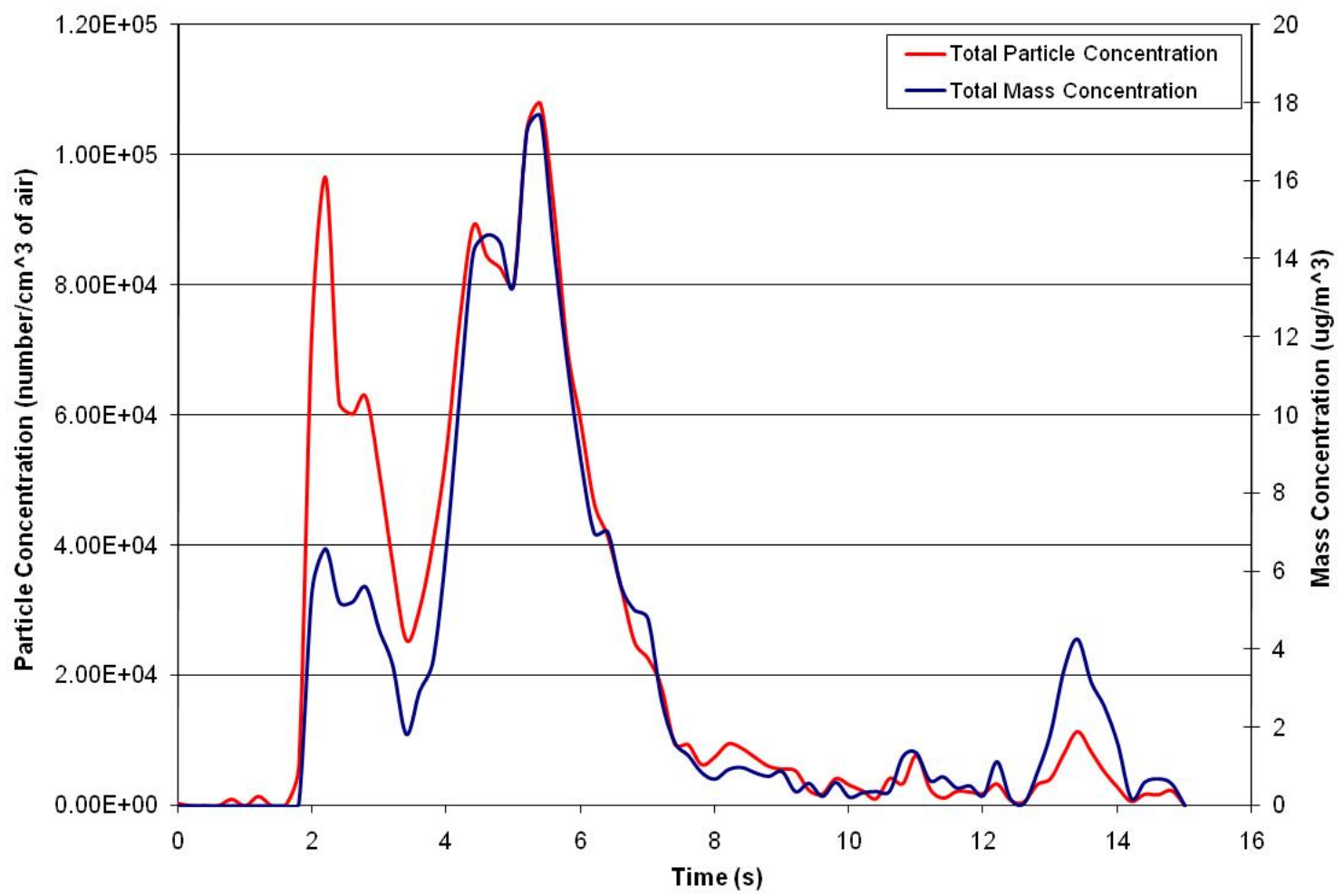

Figure 9-56: Hard acceleration gasoline drive-by with mannequin 2. 


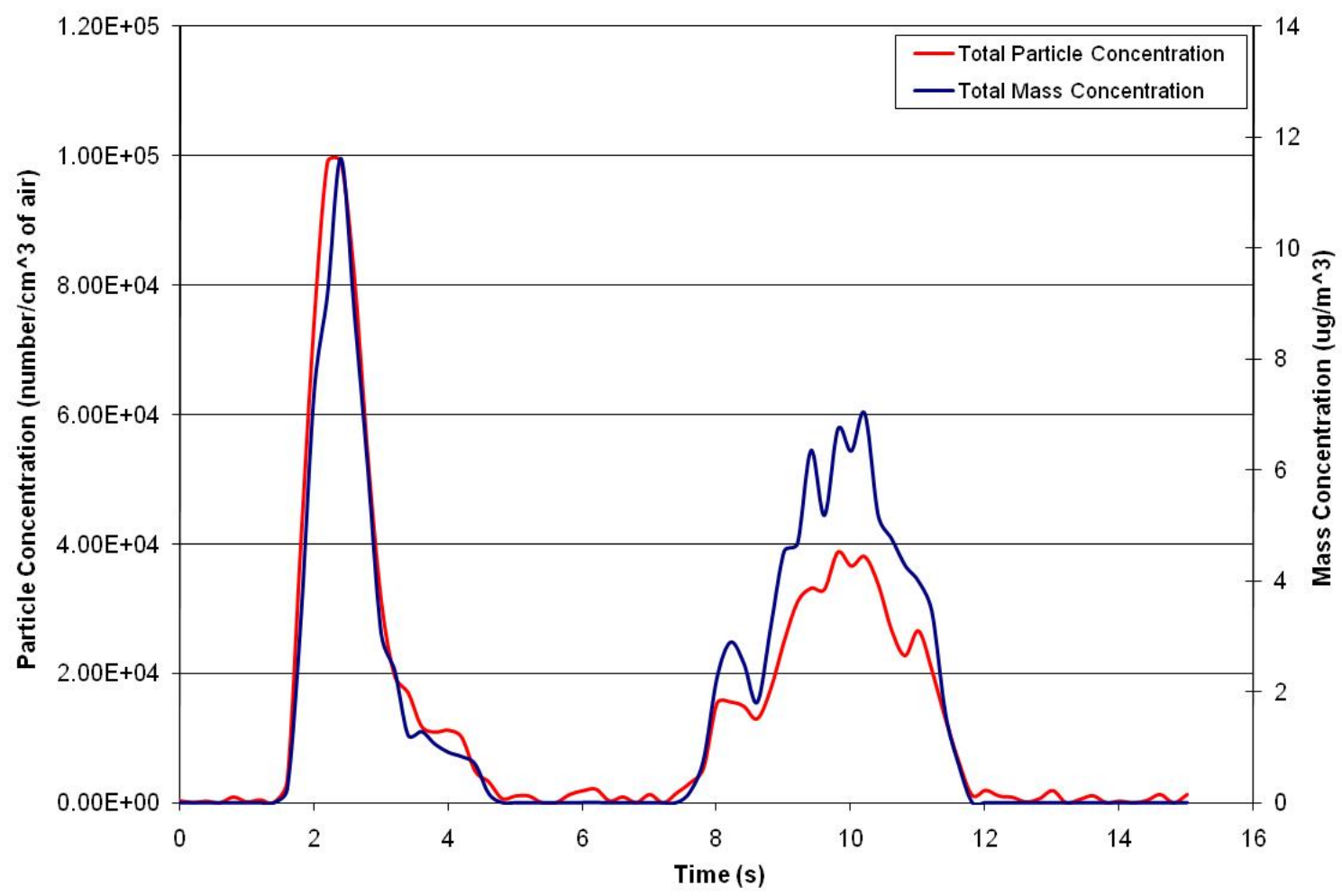

Figure 9-57: Hard acceleration gasoline drive-by with mannequin 3.

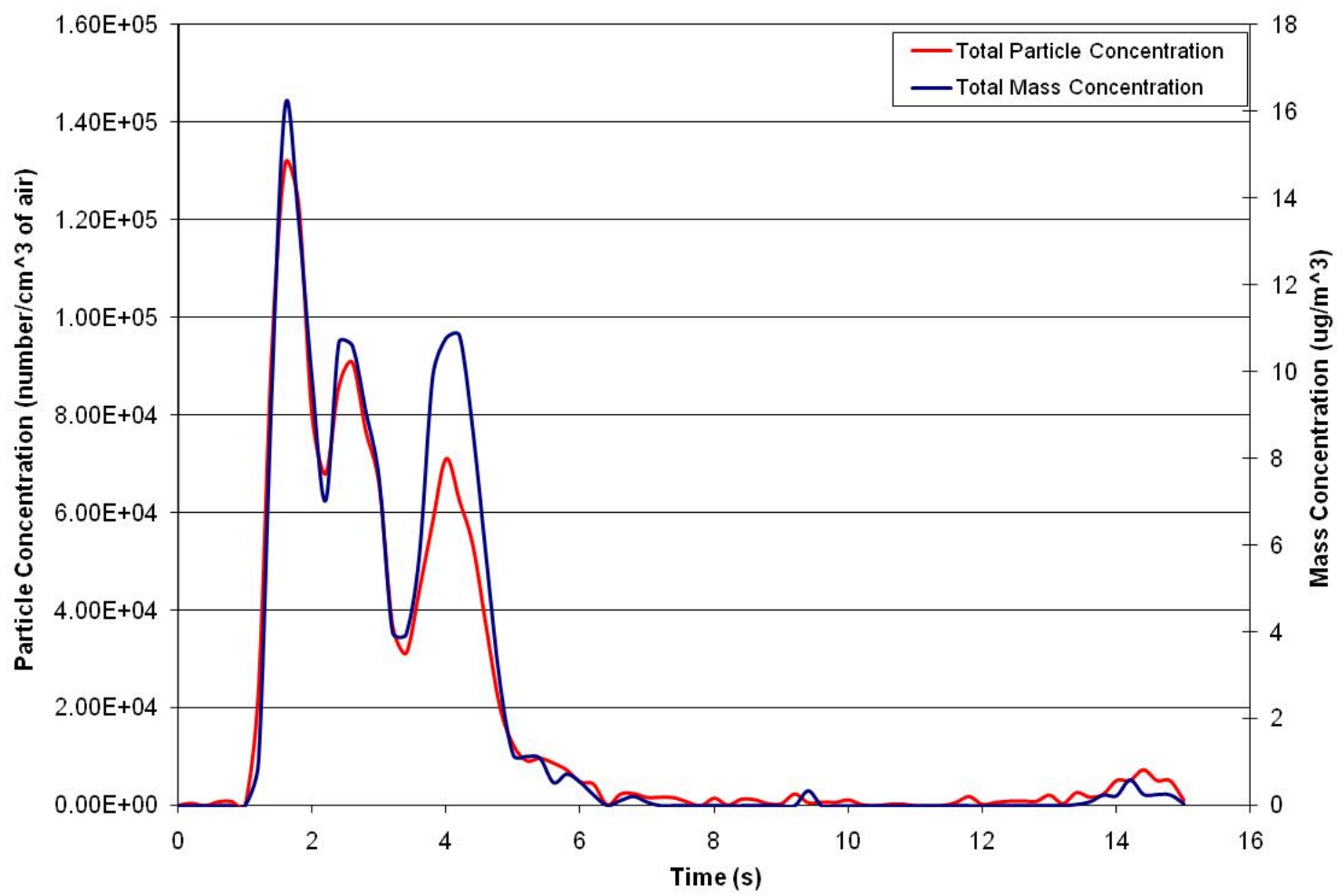

Figure 9-58: Hard acceleration gasoline drive-by with mannequin 4. 


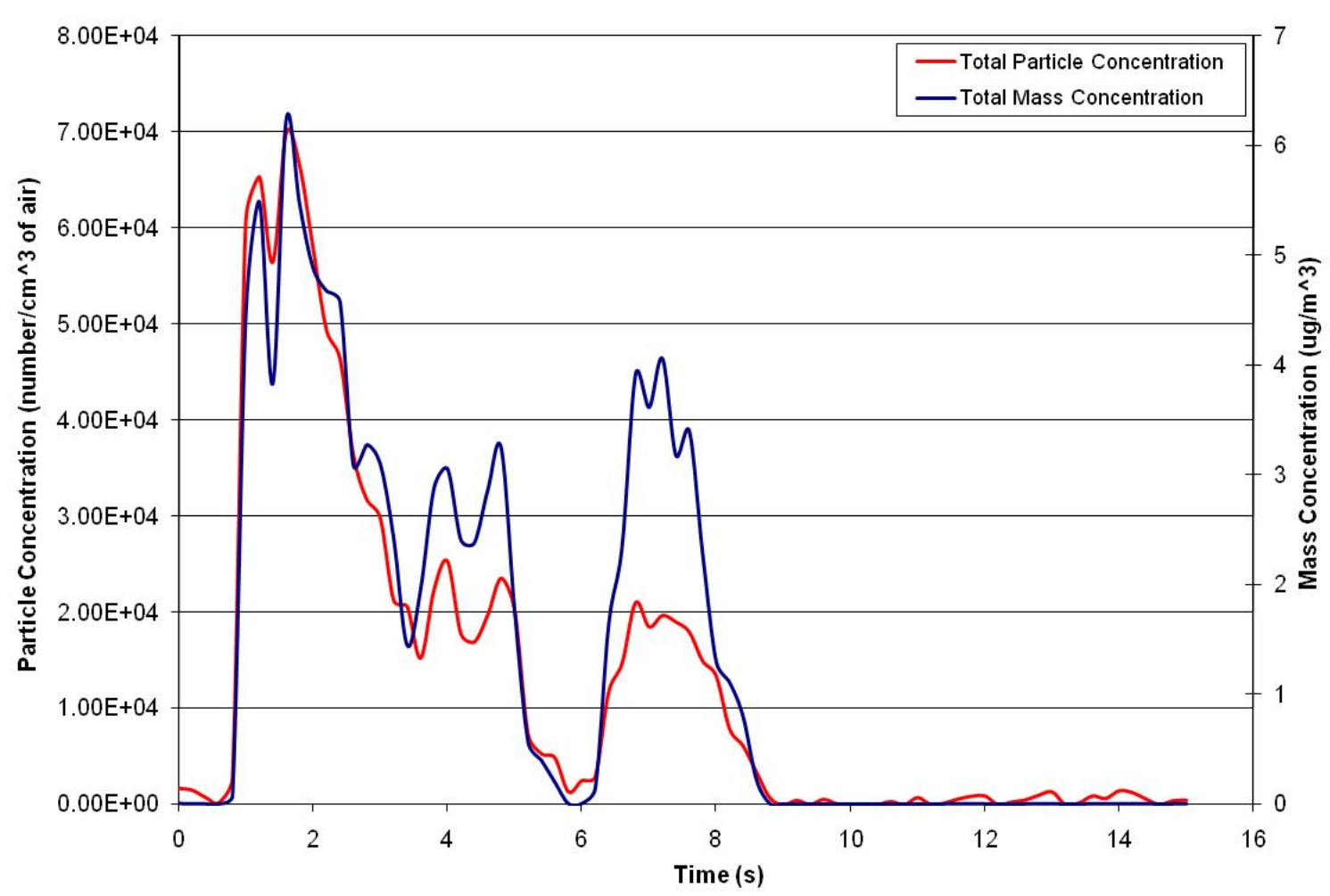

Figure 9-59: Hard acceleration gasoline drive-by with mannequin 5.

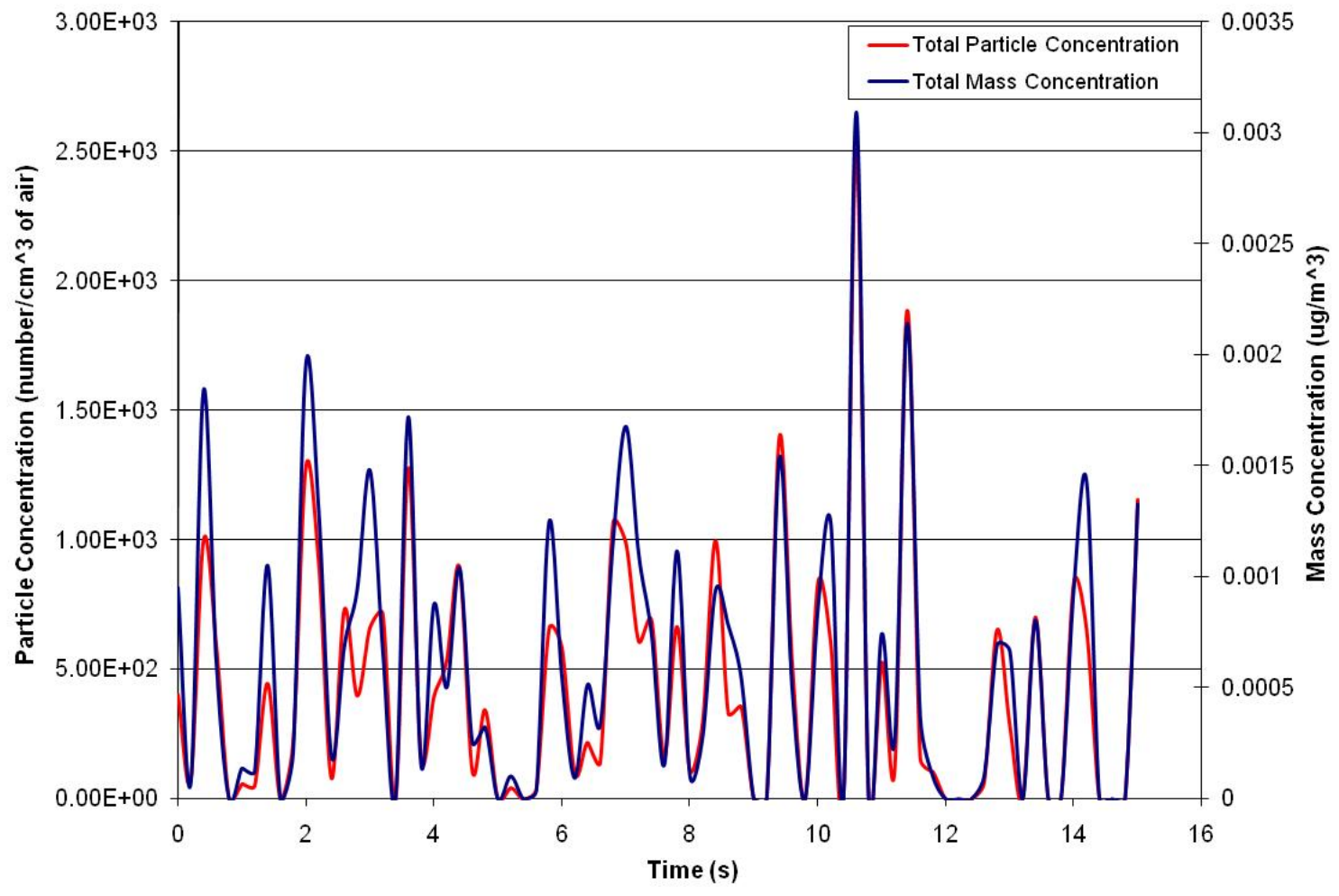

Figure 9-60: Hard acceleration gasoline drive-by with mannequin 6. 


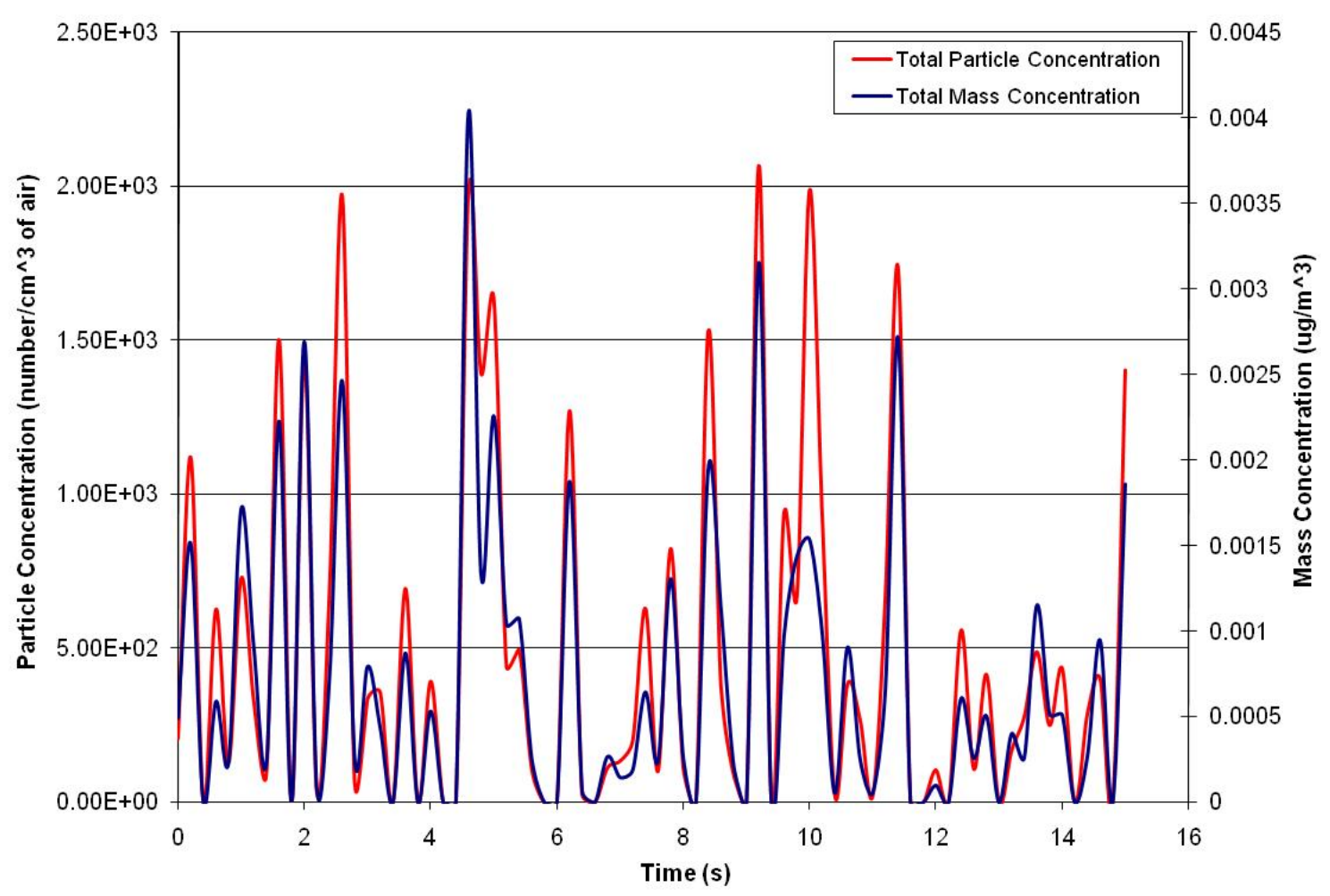

Figure 9-61: Medium acceleration gasoline drive-by with mannequin 1.

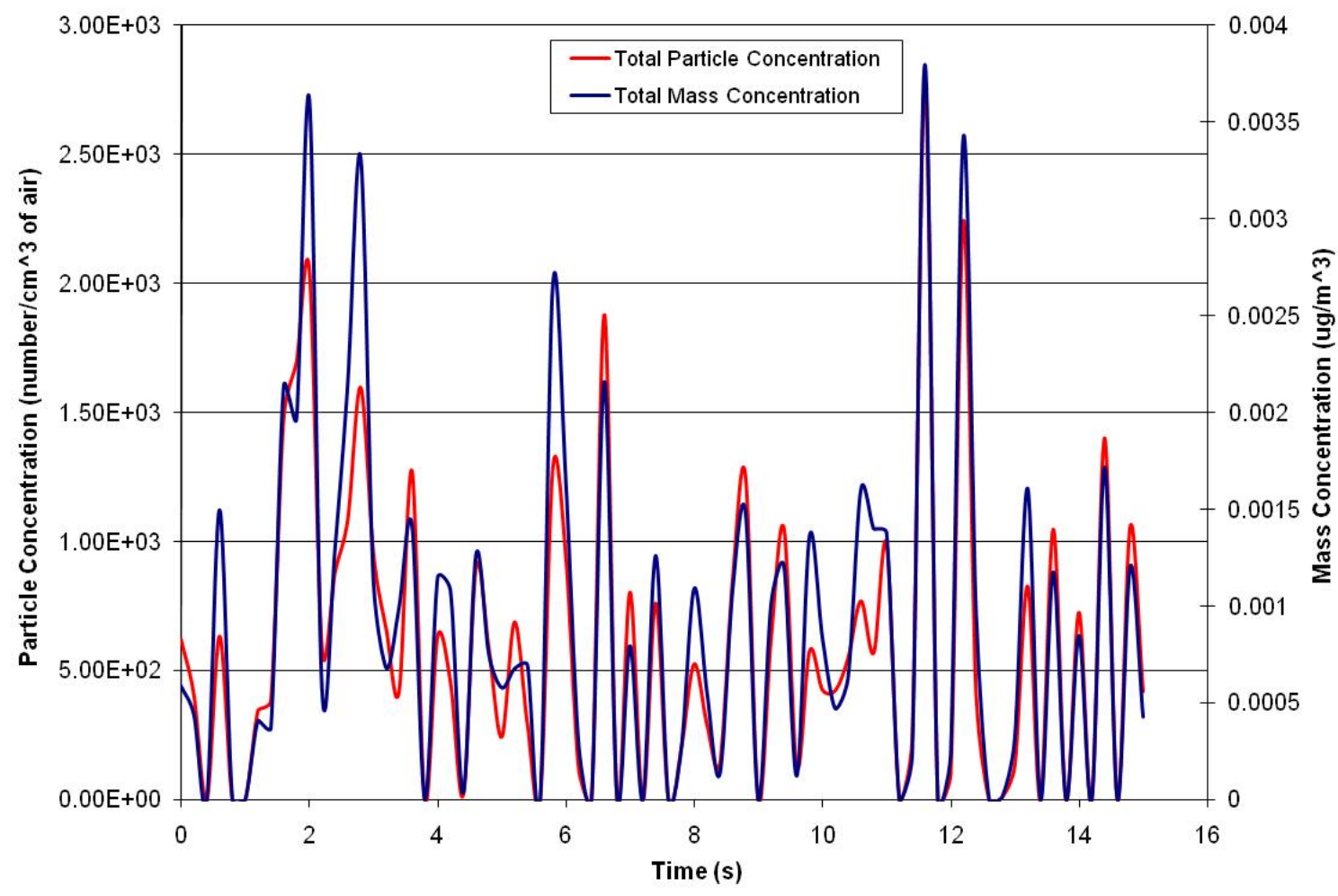

Figure 9-62: Medium acceleration gasoline drive-by with mannequin 2. 


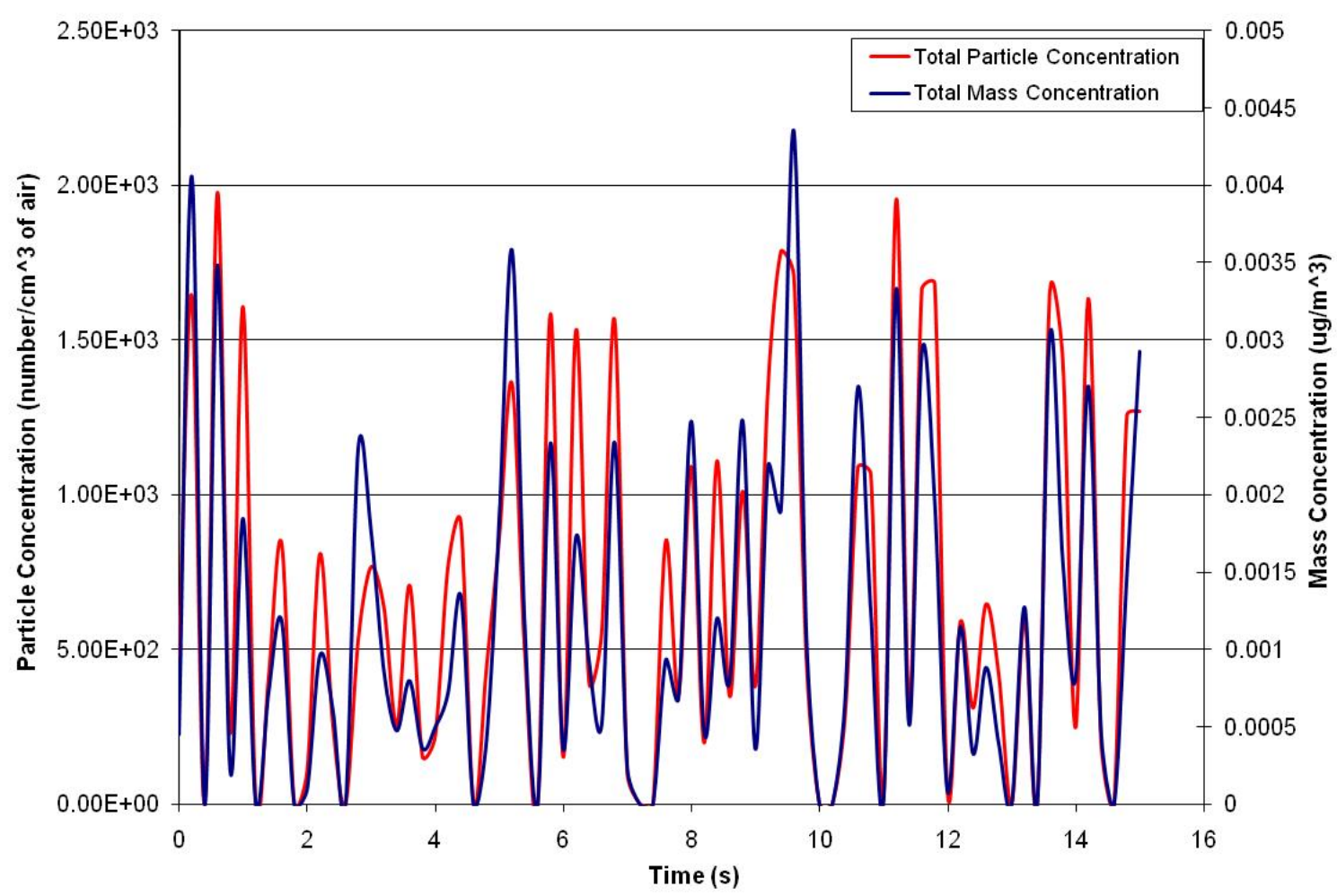

Figure 9-63: Medium acceleration gasoline drive-by with mannequin 3.

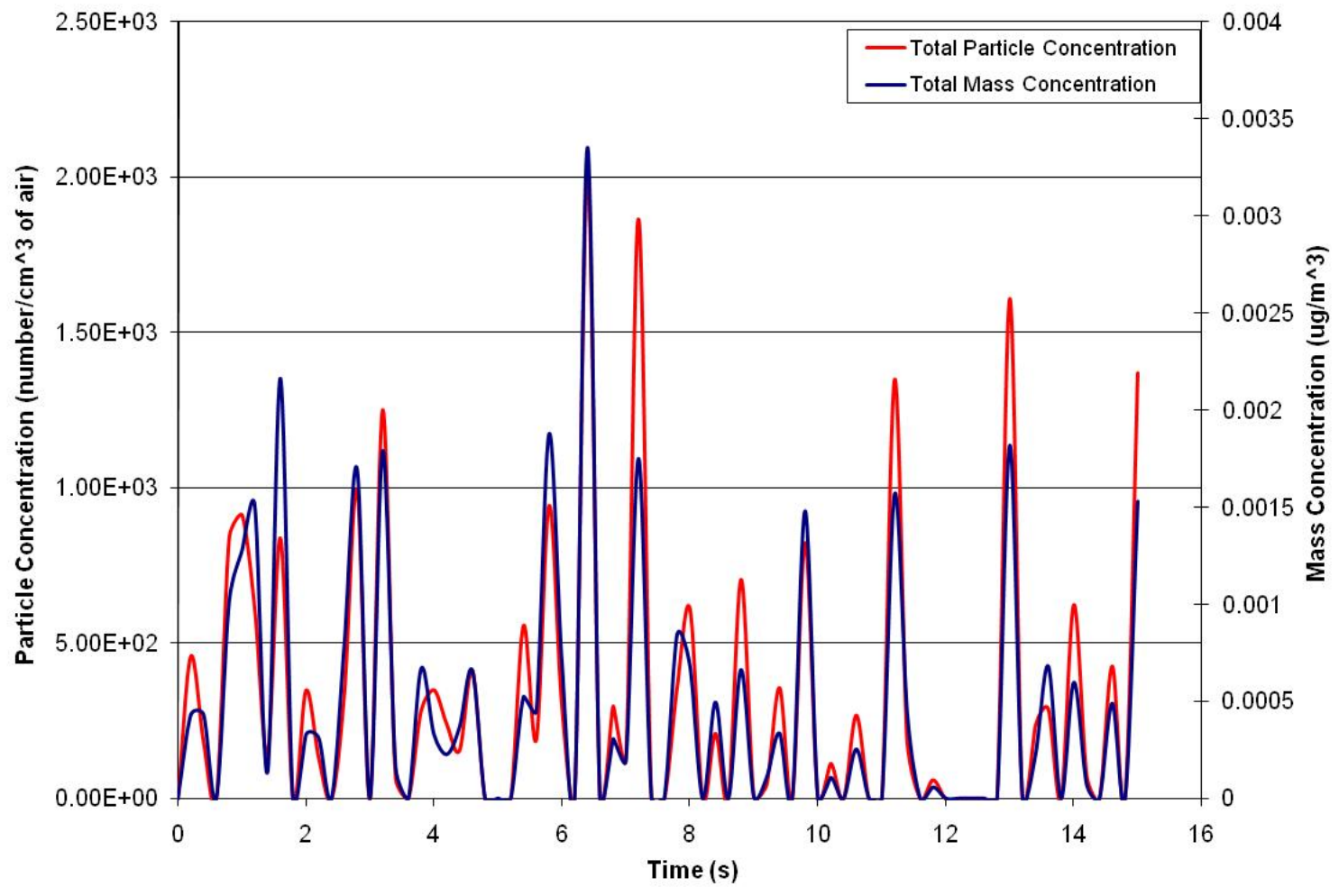

Figure 9-64: Medium acceleration gasoline drive-by with mannequin 4. 


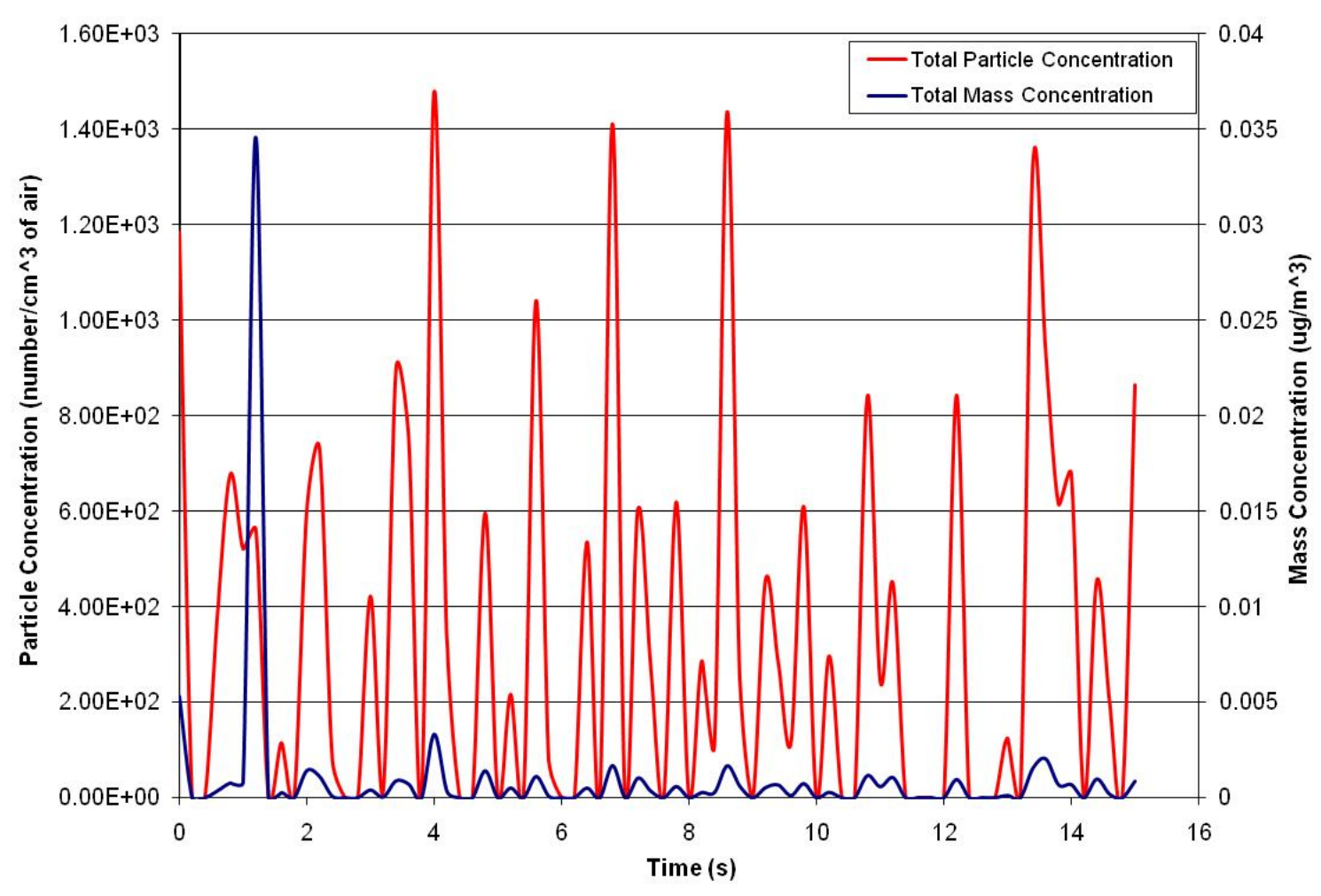

Figure 9-65: Medium acceleration gasoline drive-by with mannequin 5.

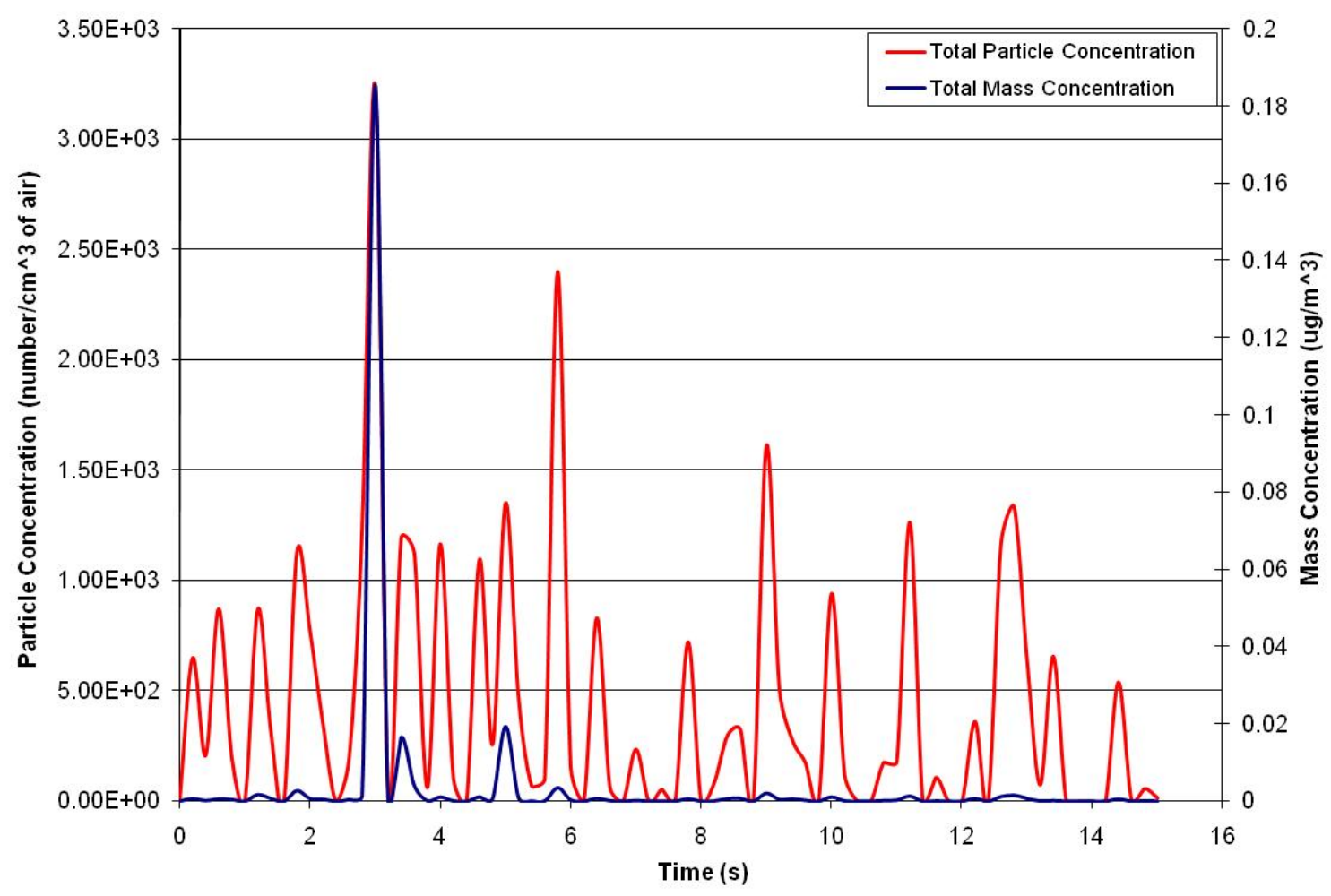

Figure 9-66: Medium acceleration gasoline drive-by with mannequin 6. 


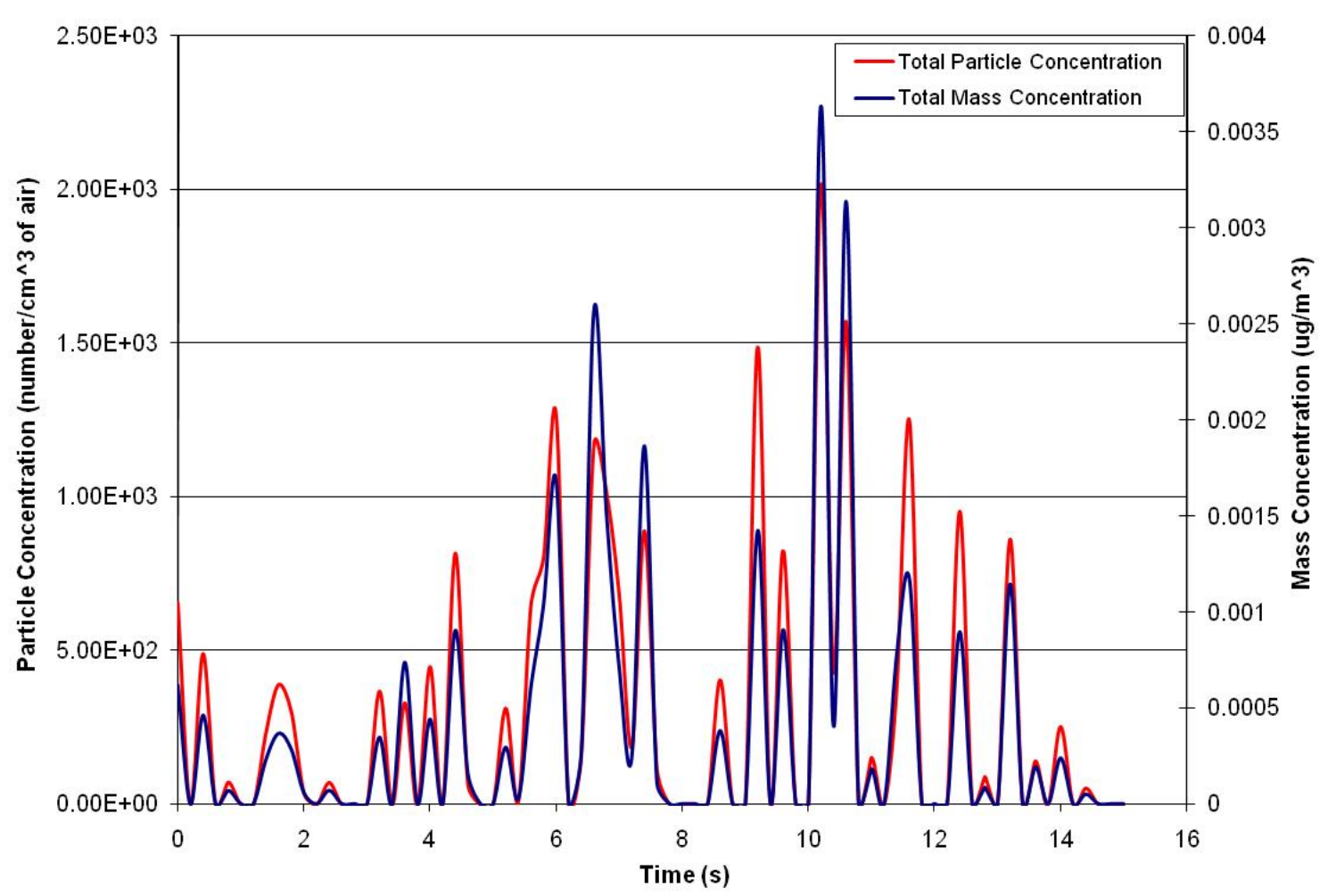

Figure 9-67: Cruising gasoline drive-by with mannequin 1.

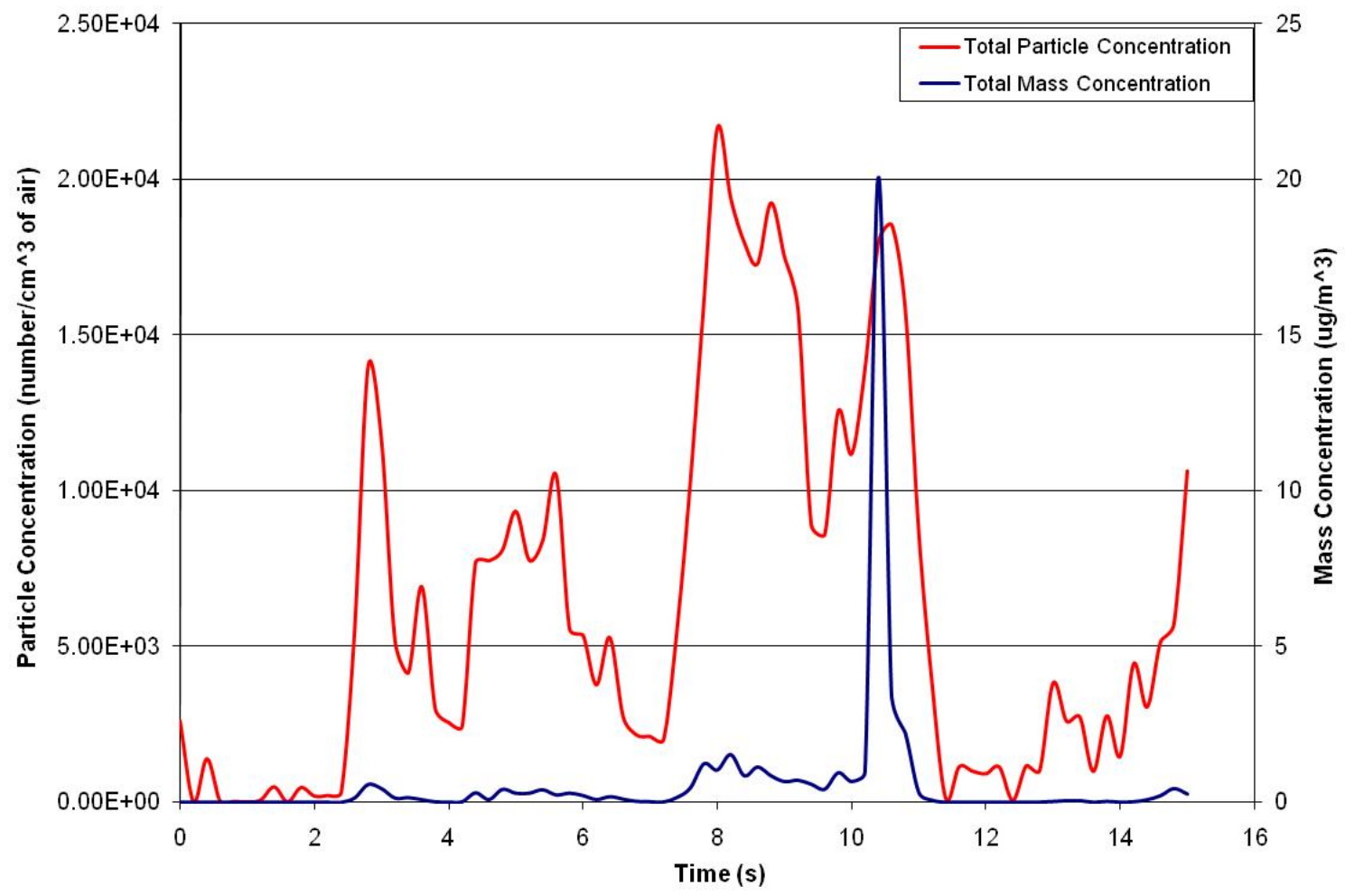

Figure 9-68: Cruising gasoline drive-by with mannequin 2. 


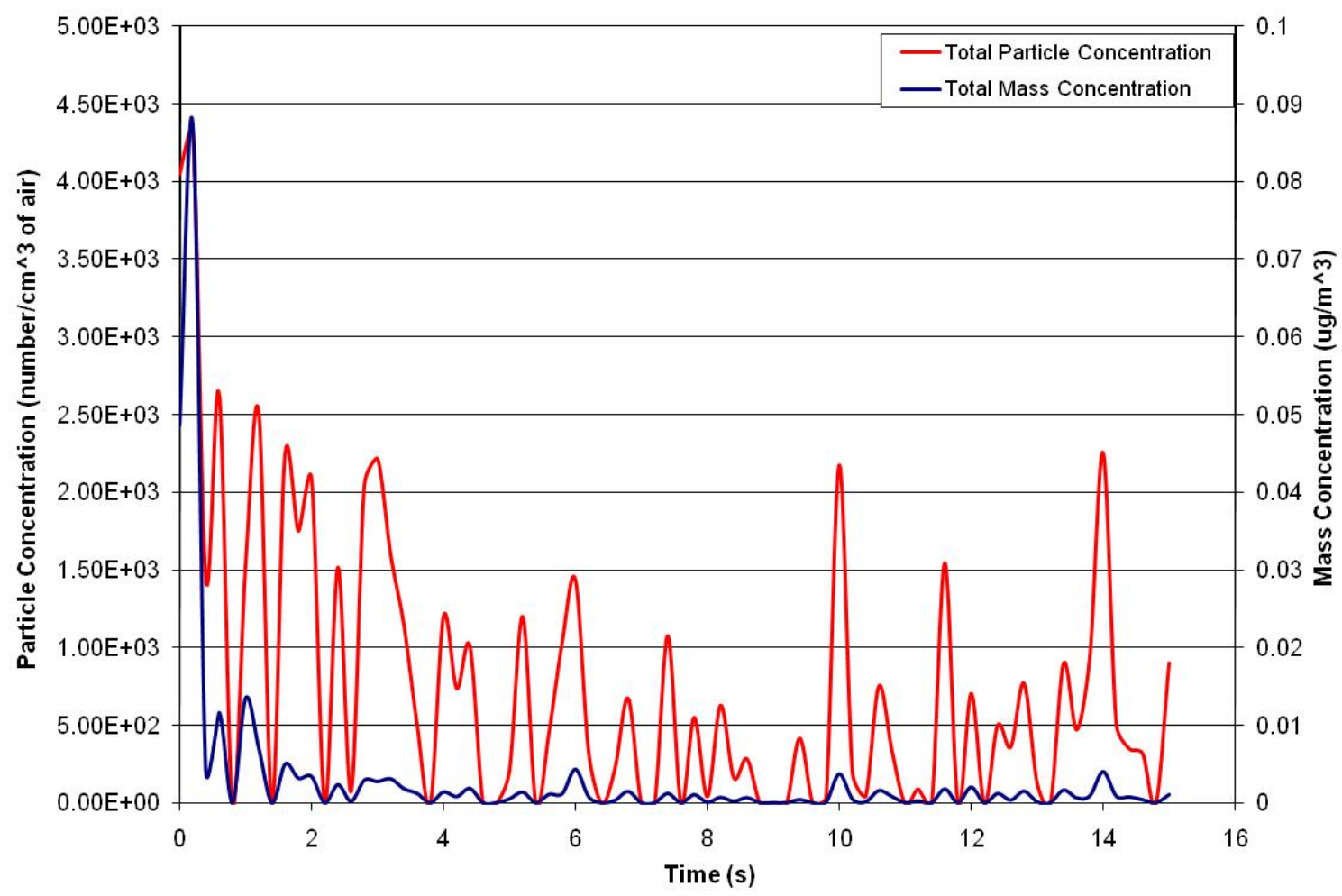

Figure 9-69: Cruising gasoline drive-by with mannequin 3.

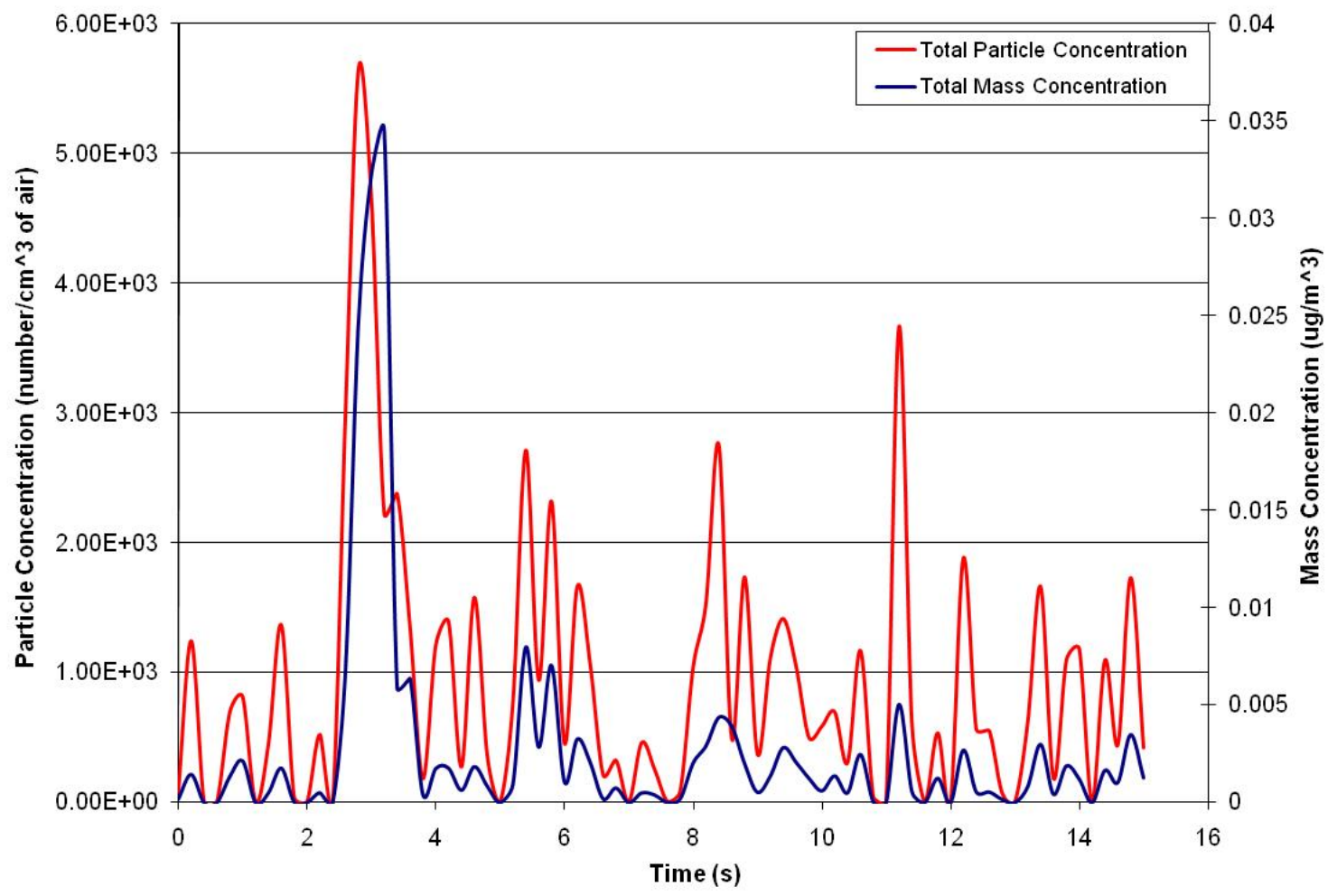

Figure 9-70: Cruising gasoline drive-by with mannequin 4 . 


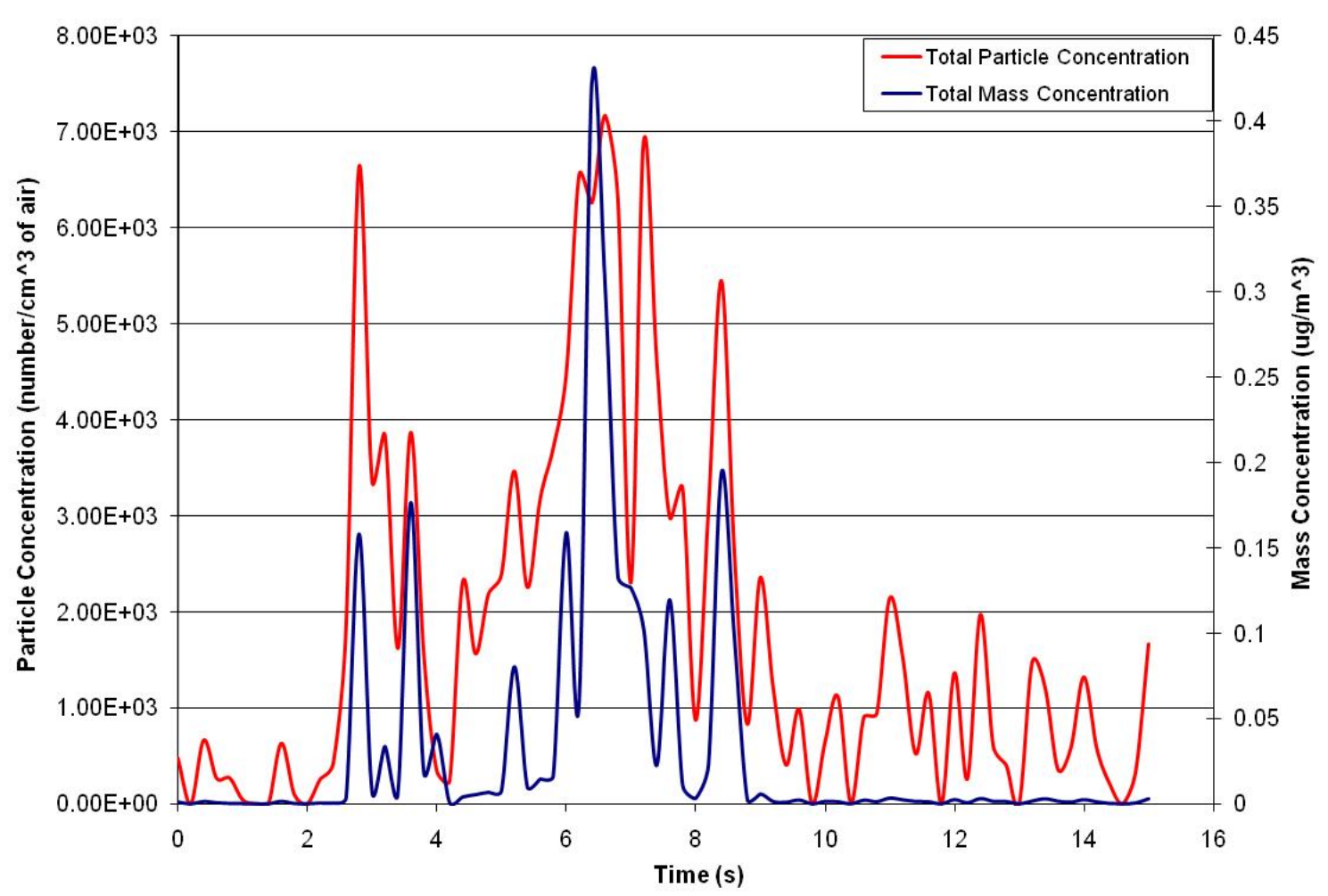

Figure 9-71: Cruising gasoline drive-by with mannequin 5.

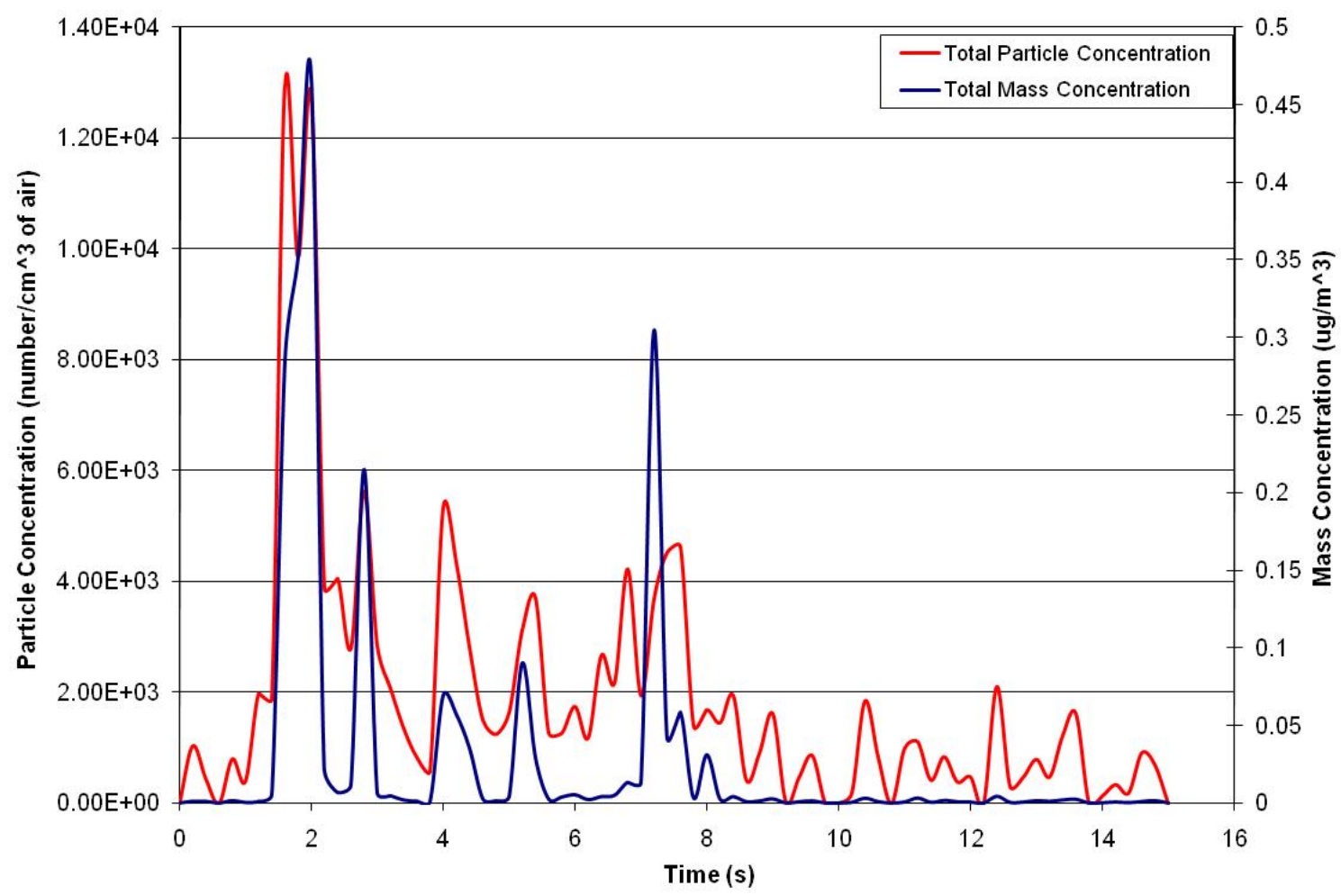

Figure 9-72: Cruising gasoline drive-by with mannequin 6. 


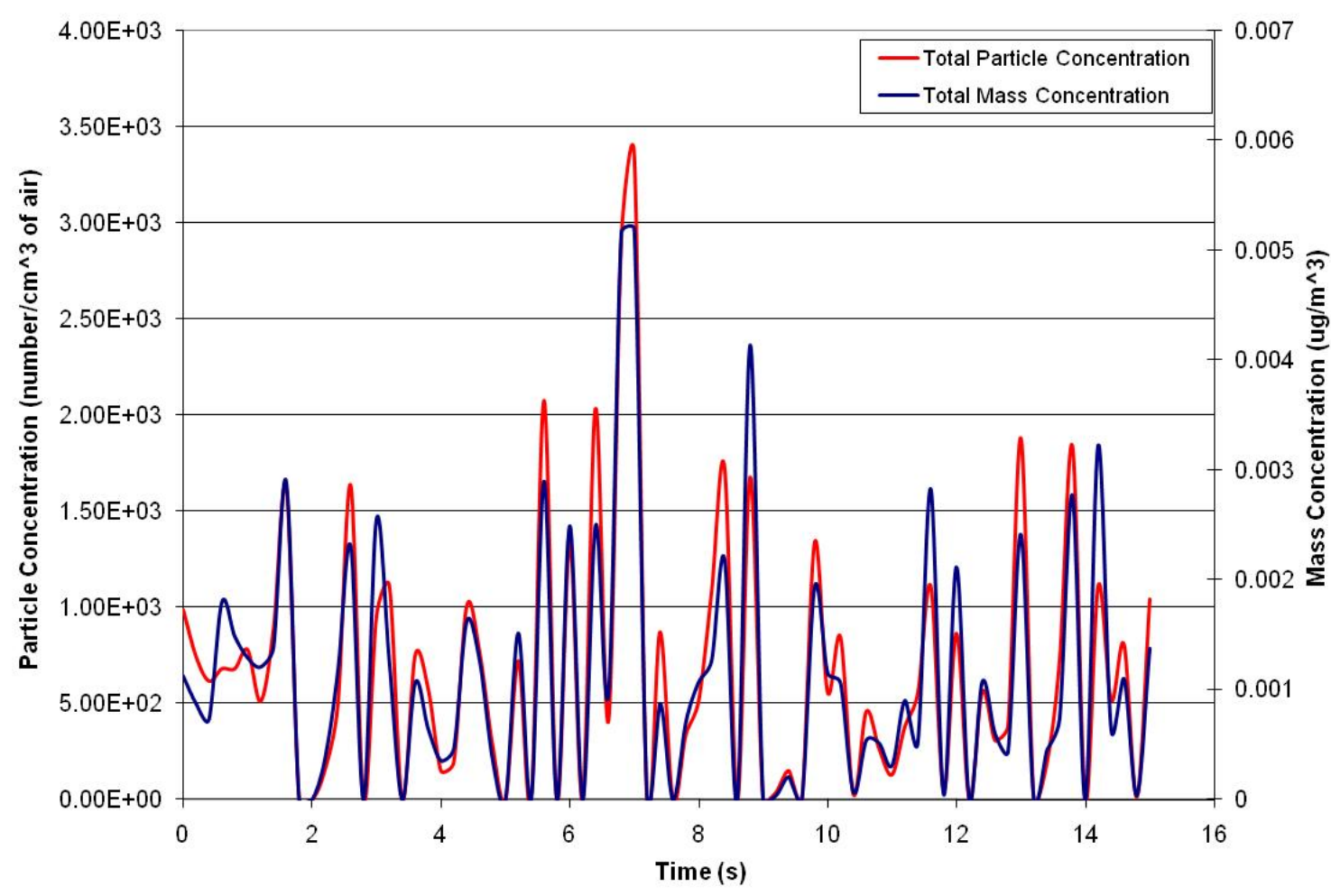

Figure 9-73: Hard acceleration gasoline drive-by without mannequin 1.

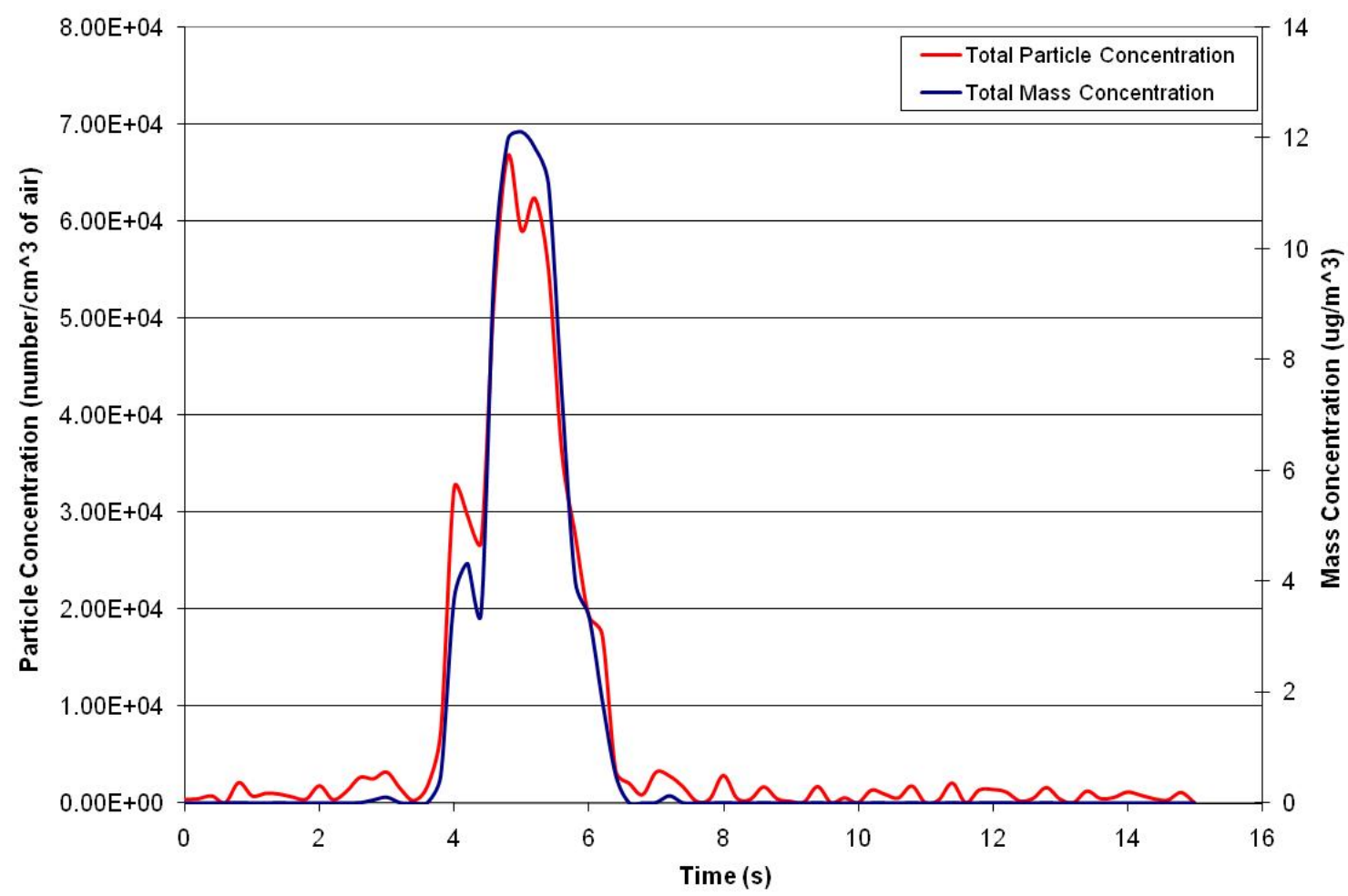

Figure 9-74: Hard acceleration gasoline drive-by without mannequin 2. 


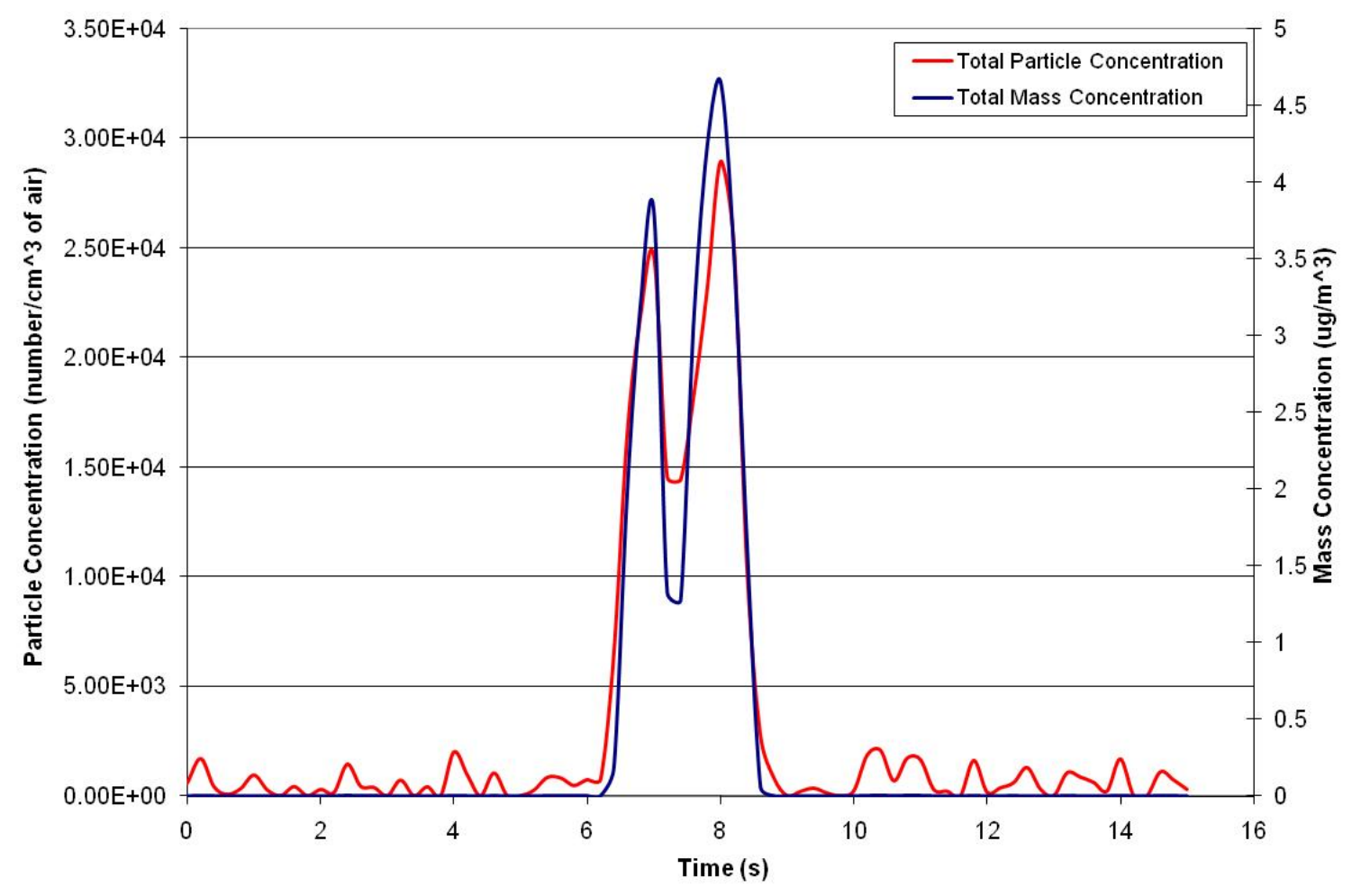

Figure 9-75: Hard acceleration gasoline drive-by without mannequin 3.

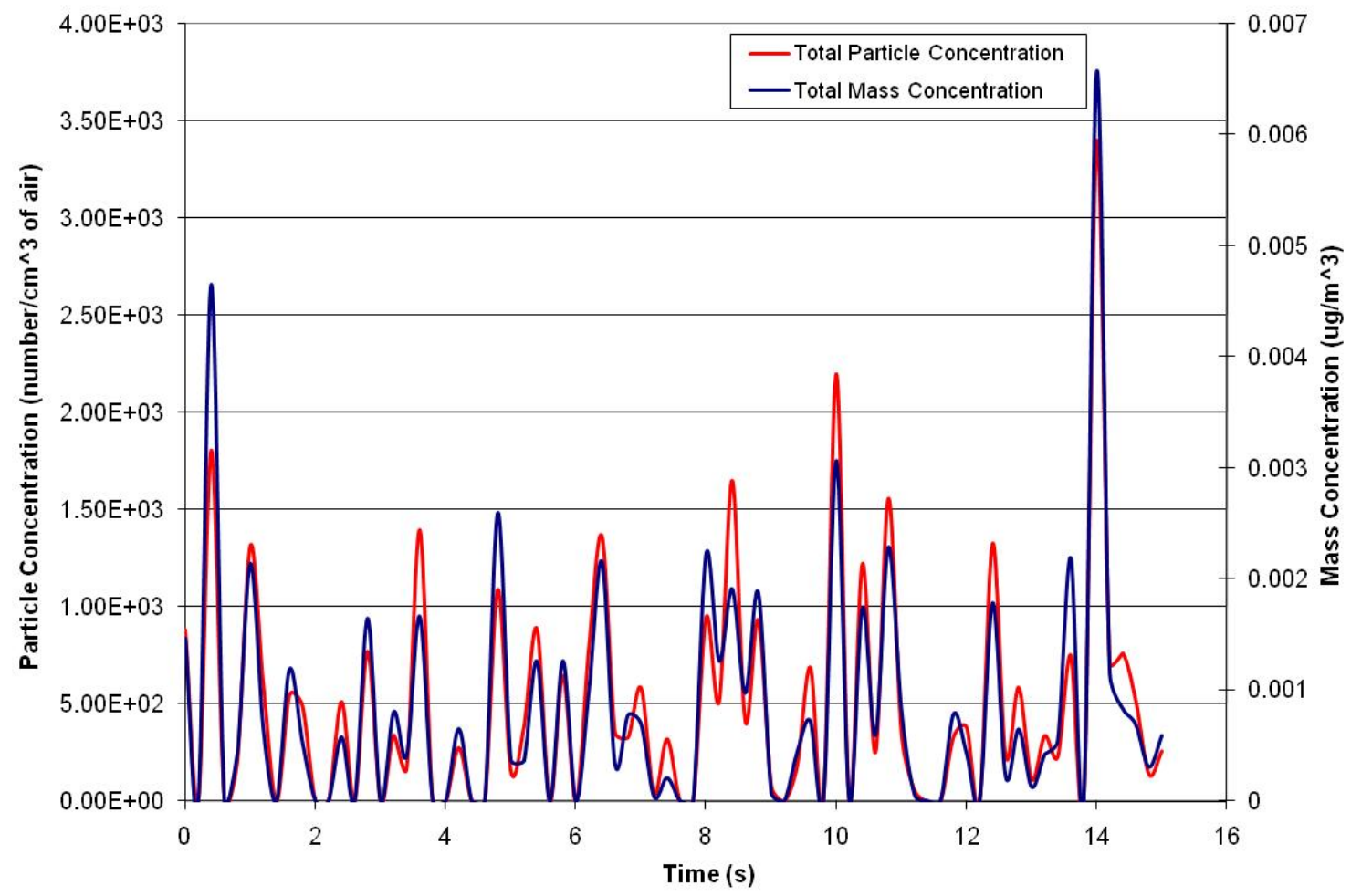

Figure 9-76: Hard acceleration gasoline drive-by without mannequin 4. 


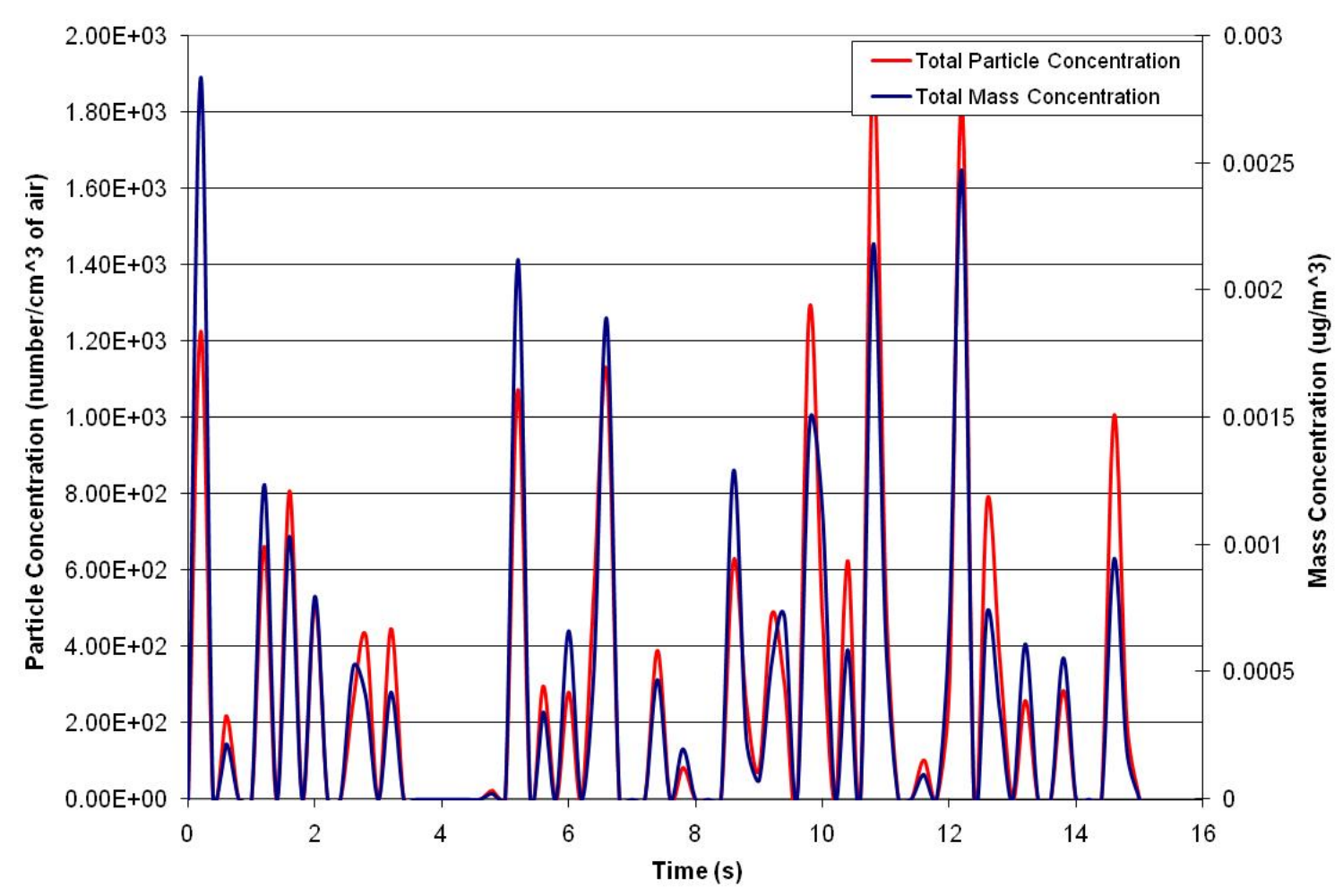

Figure 9-77: Hard acceleration gasoline drive-by without mannequin 5.

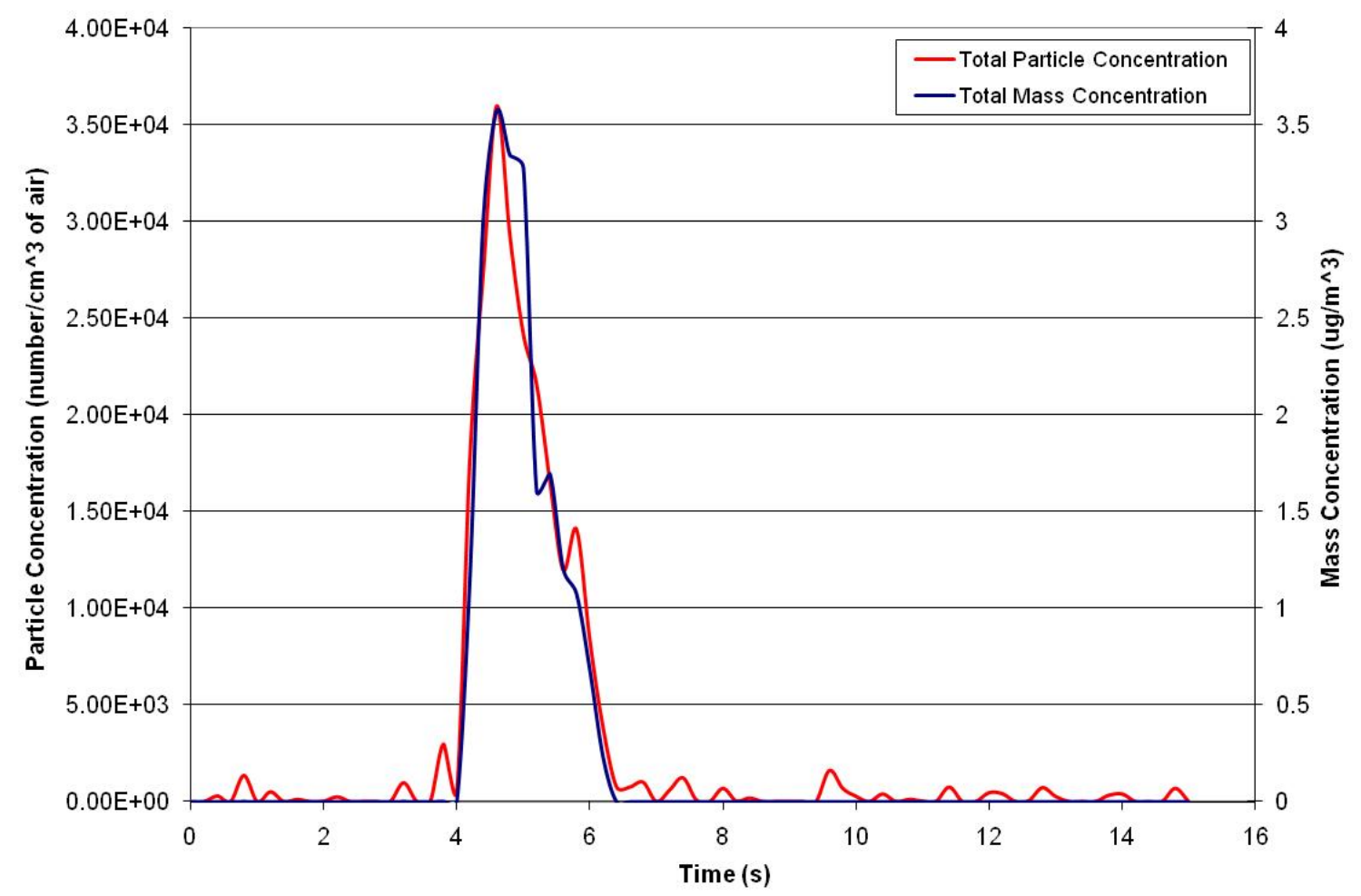

Figure 9-78: Hard acceleration gasoline drive-by without mannequin 6. 


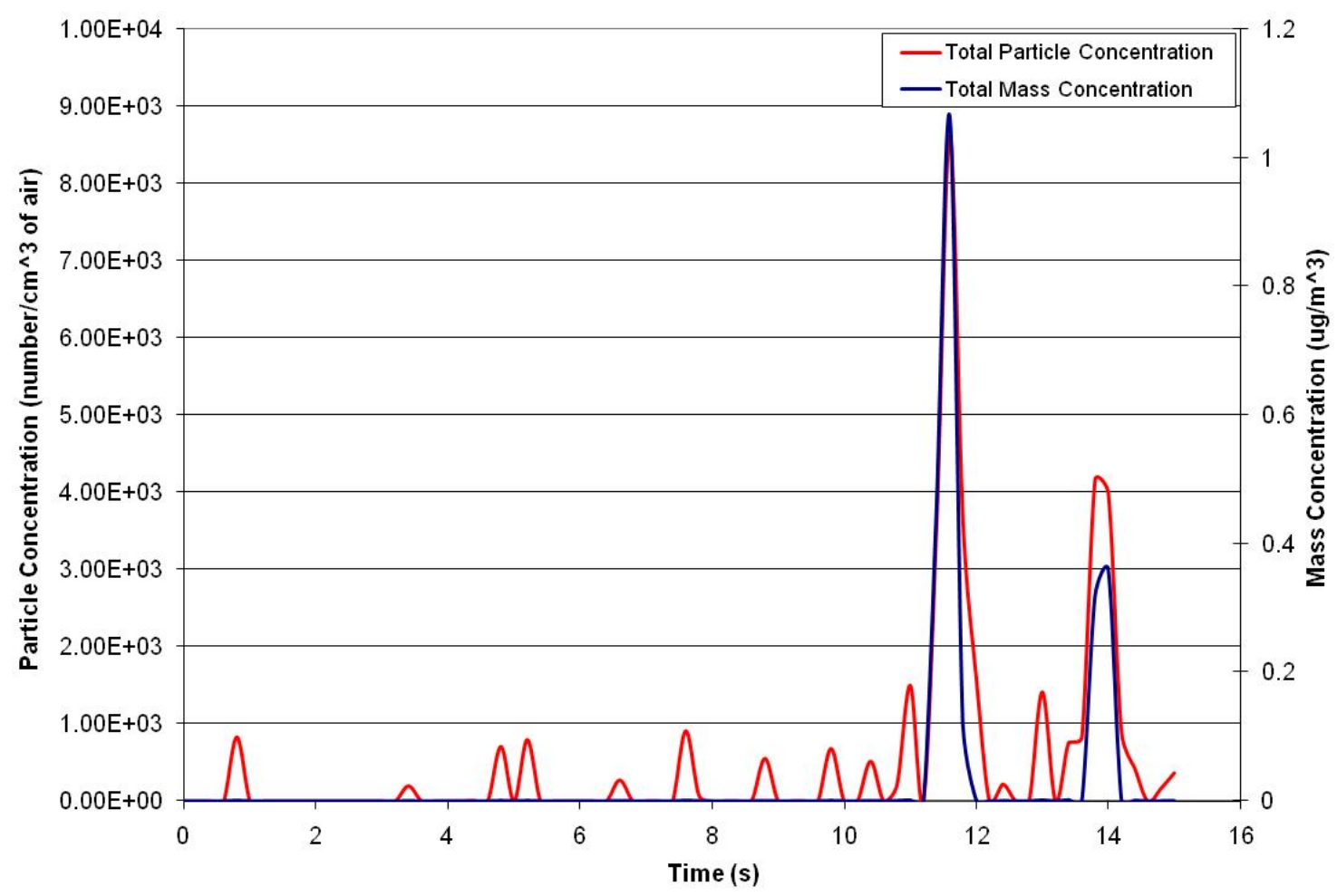

Figure 9-79: Medium acceleration gasoline drive-by without mannequin 1.

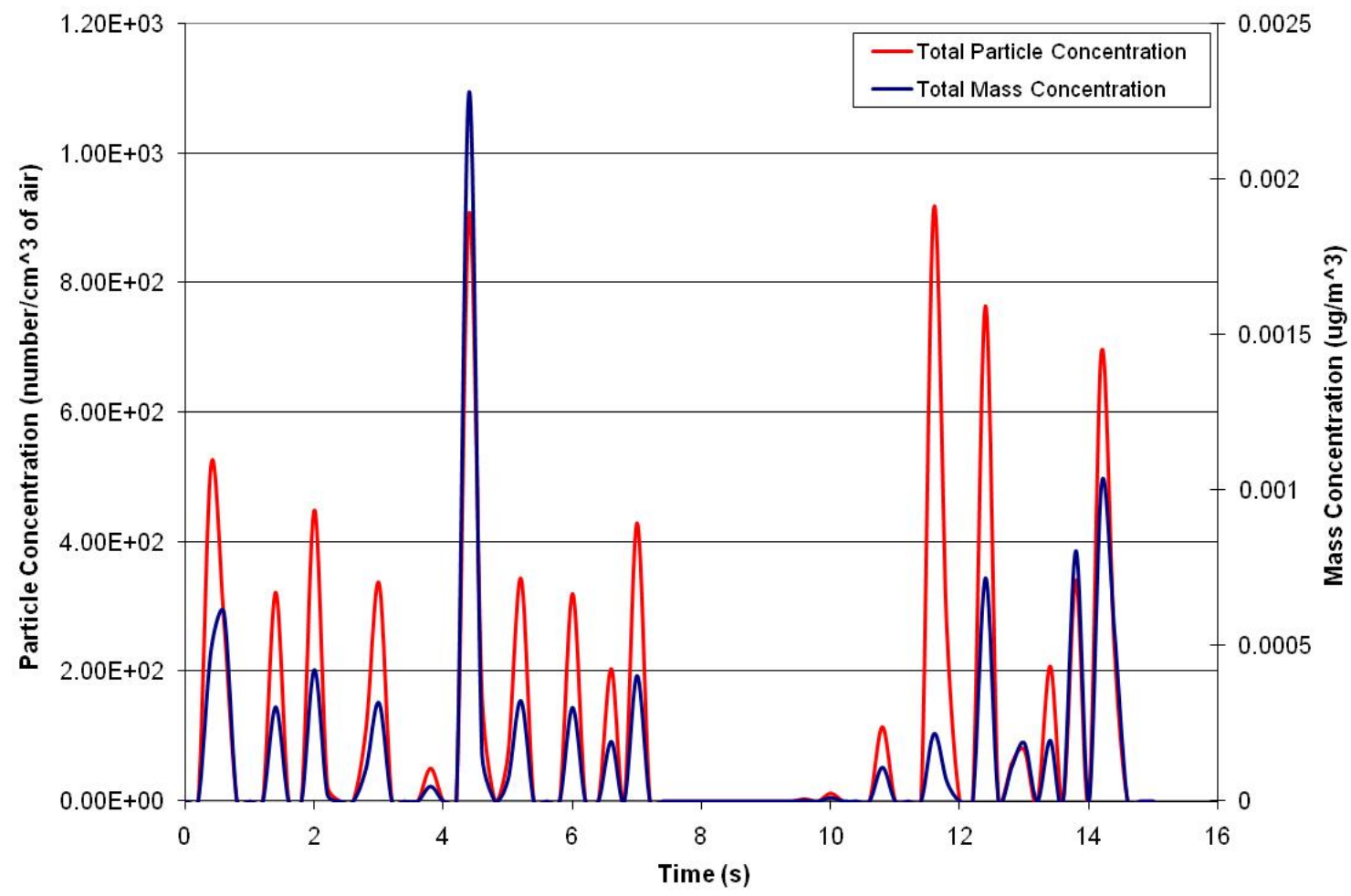

Figure 9-80: Medium acceleration gasoline drive-by without mannequin 2. 


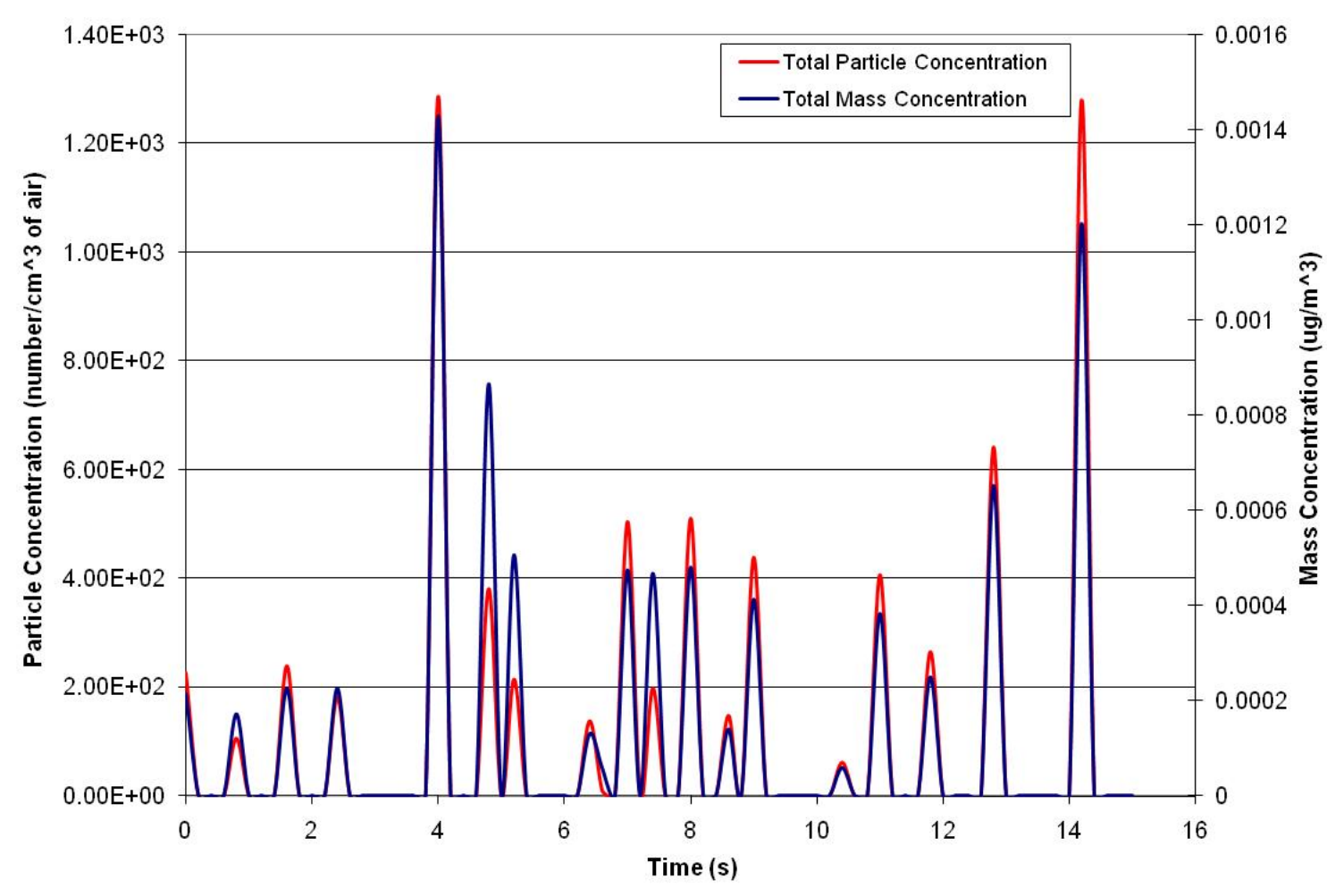

Figure 9-81: Medium acceleration gasoline drive-by without mannequin 3.

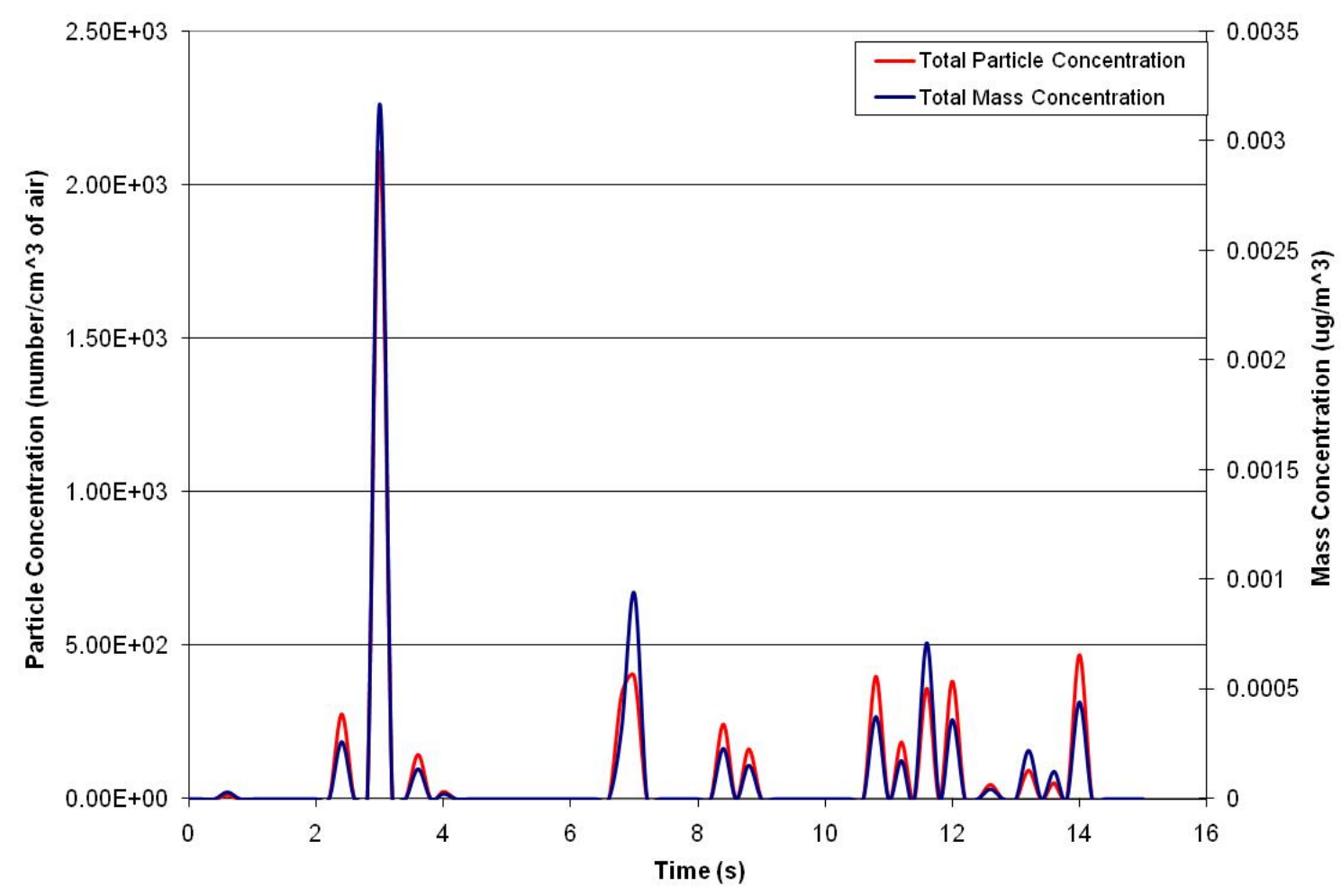

Figure 9-82: Medium acceleration gasoline drive-by without mannequin 4. 


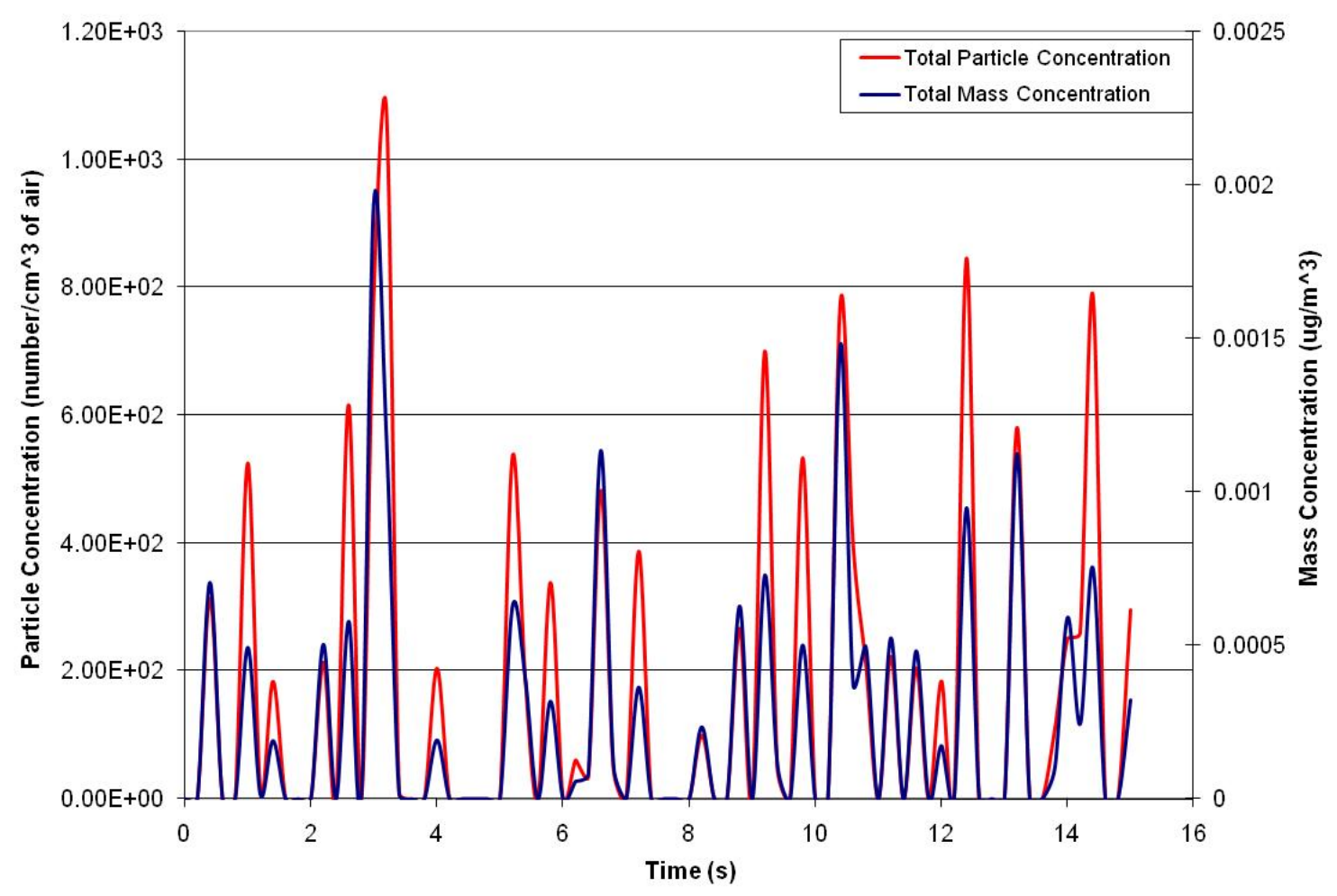

Figure 9-83: Medium acceleration gasoline drive-by without mannequin 5.

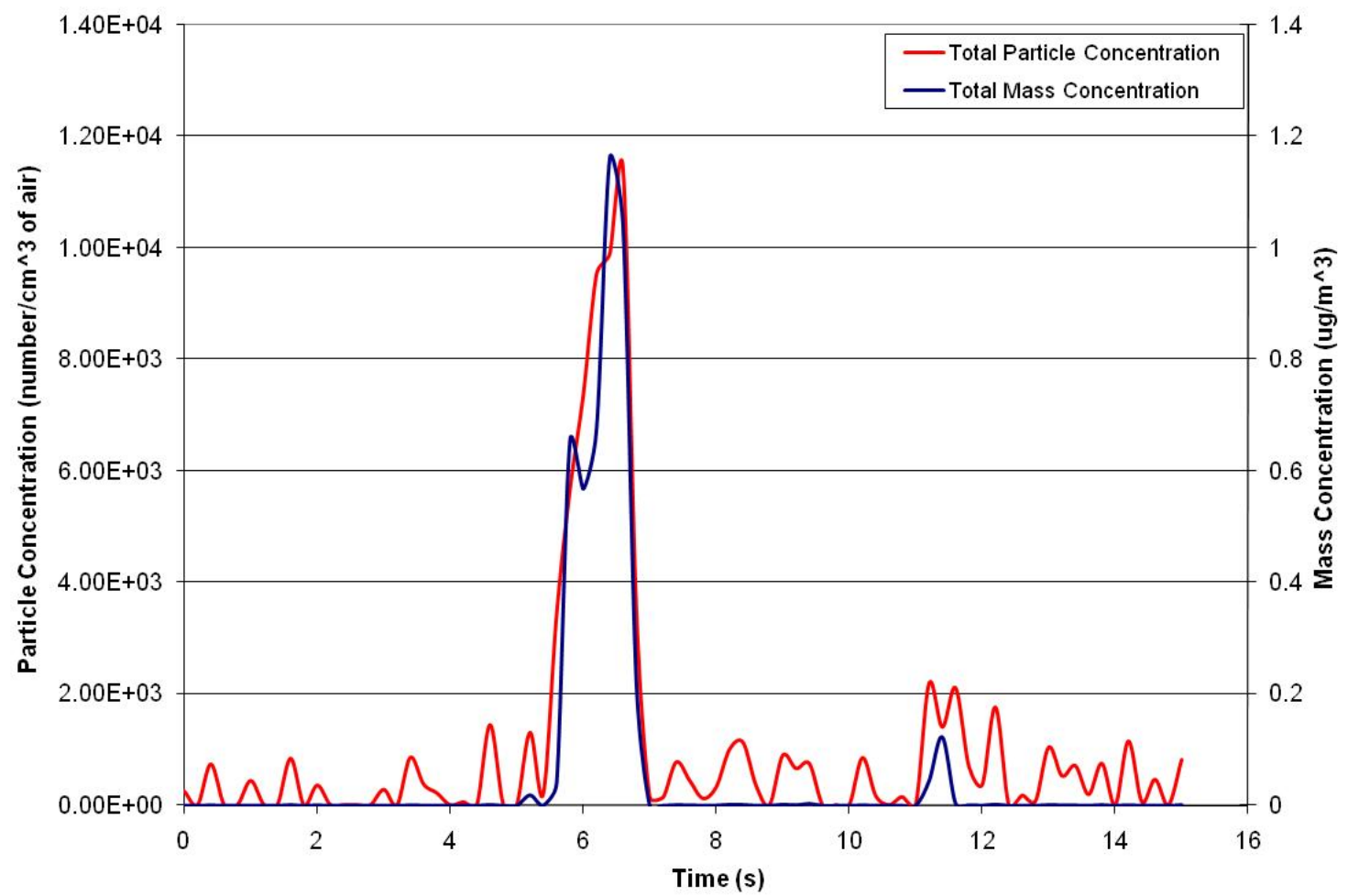

Figure 9-84: Medium acceleration gasoline drive-by without mannequin 6. 


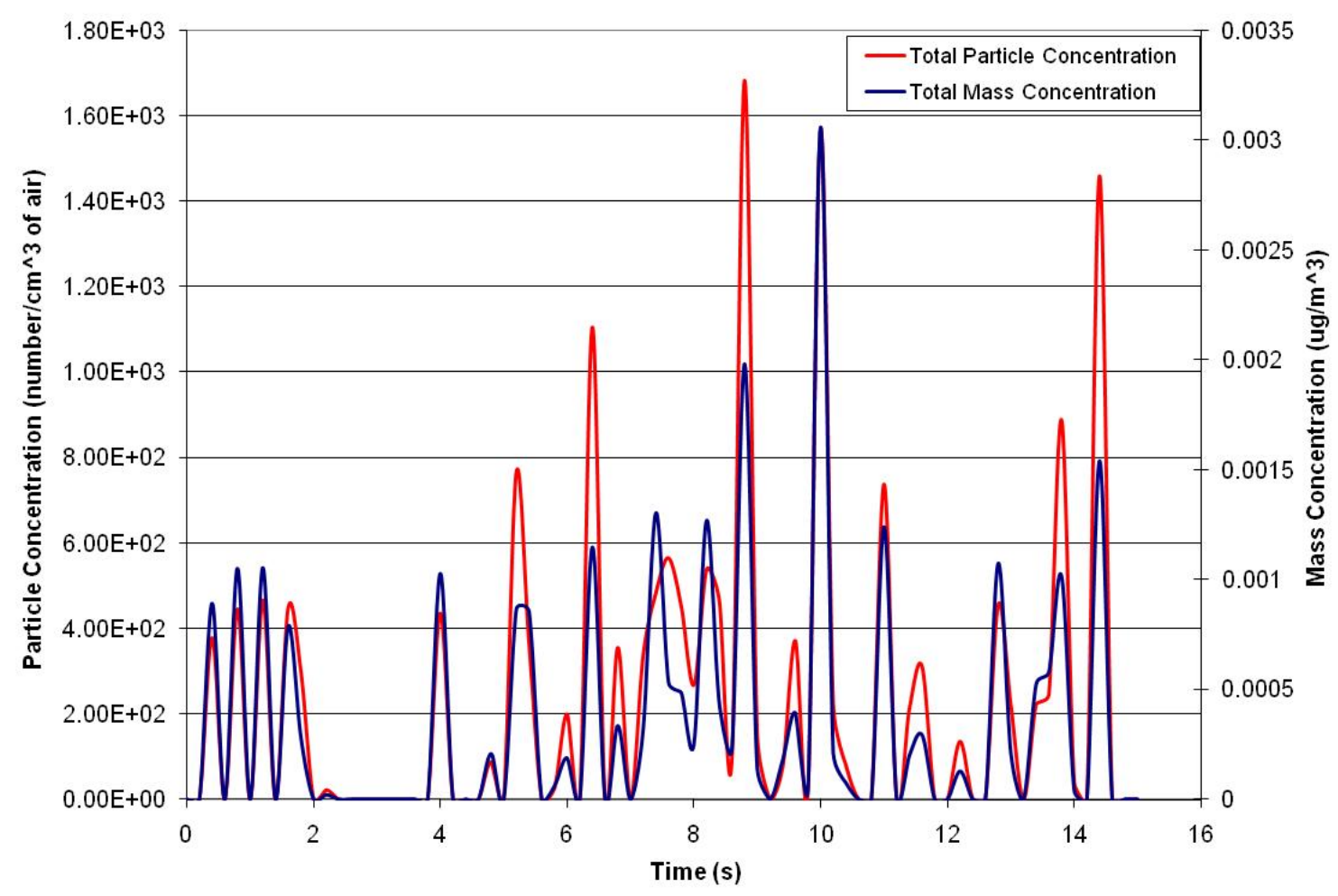

Figure 9-85: Cruising gasoline drive-by without mannequin 1.

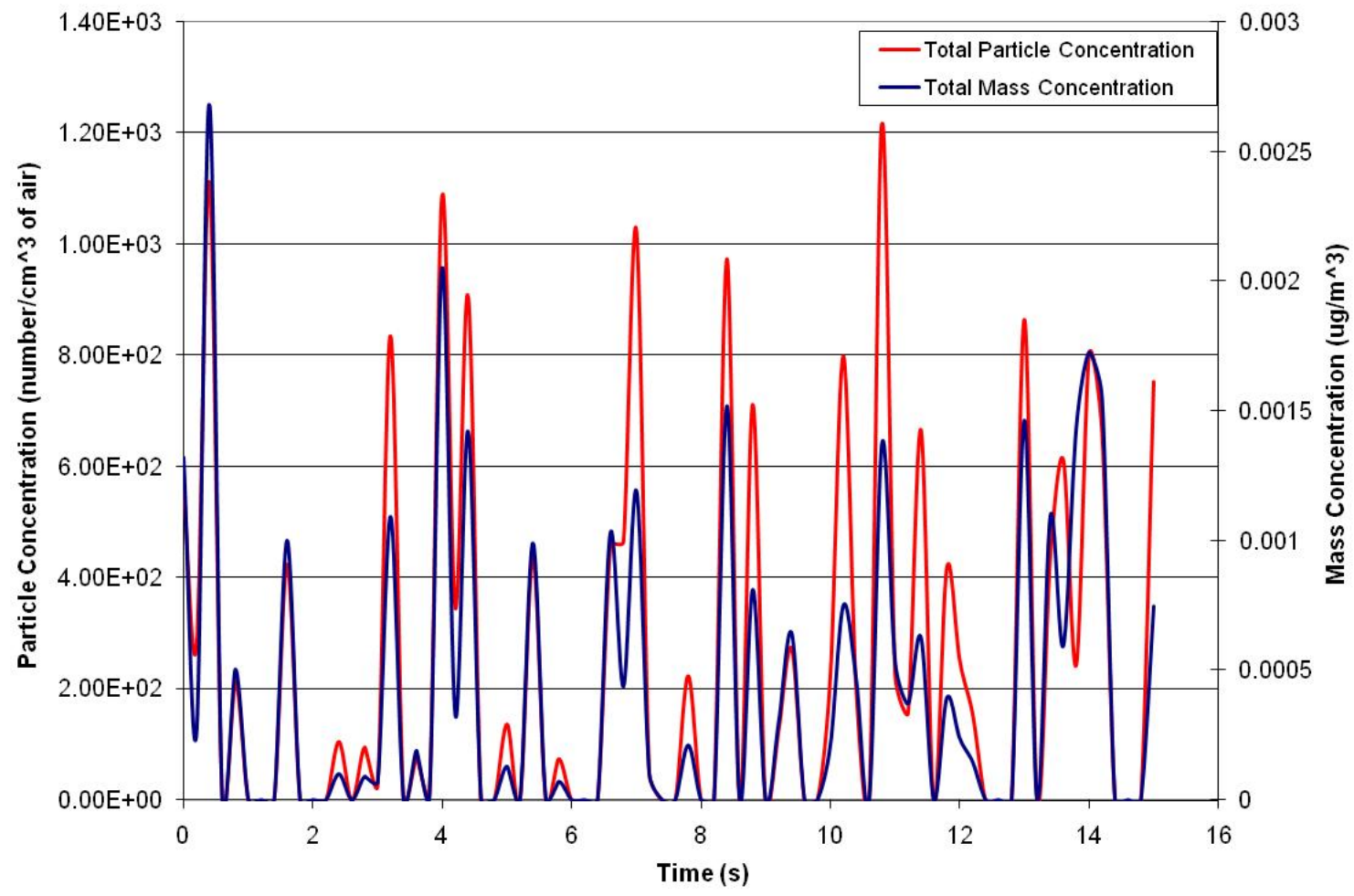

Figure 9-86: Cruising gasoline drive-by without mannequin 2. 


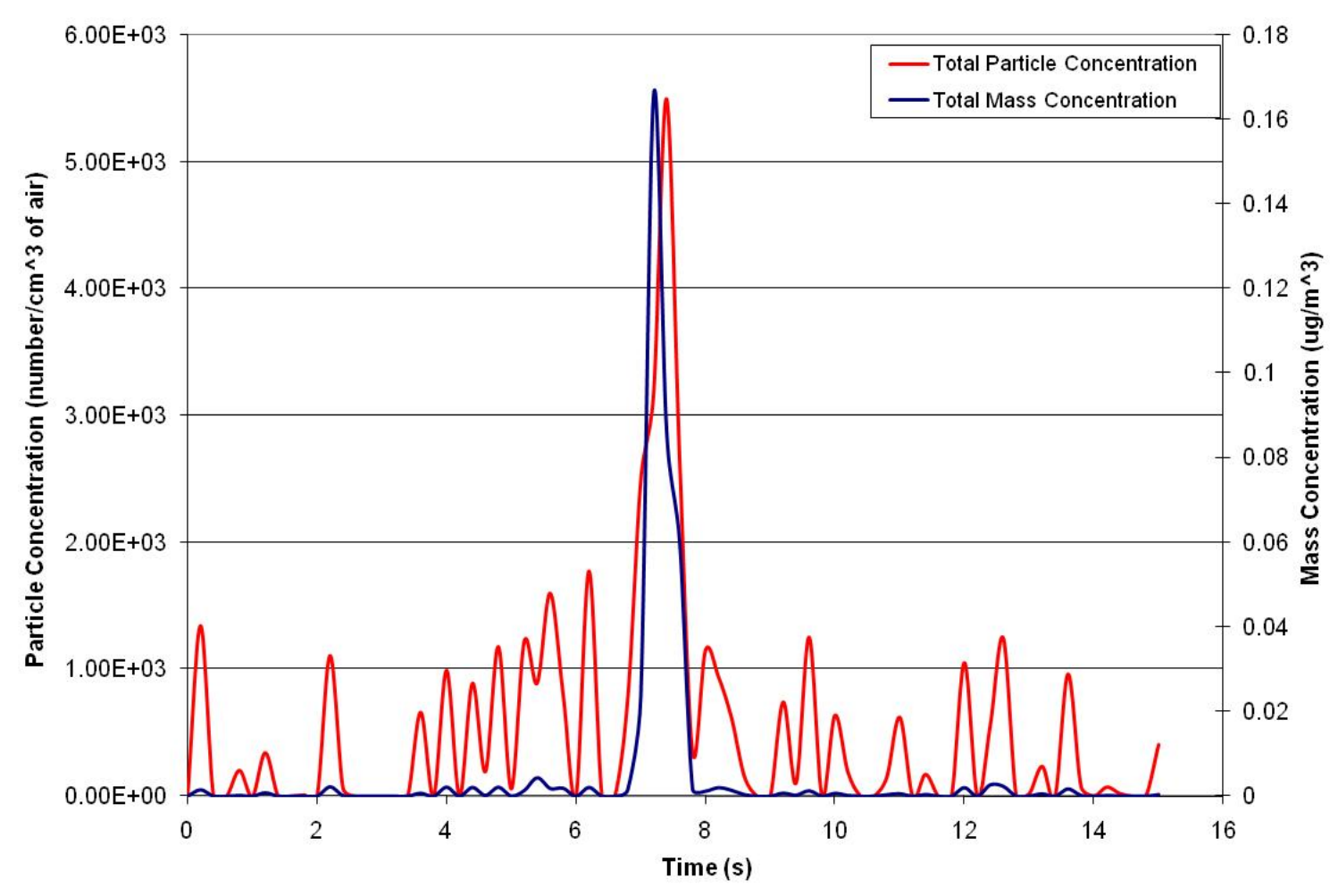

Figure 9-87: Cruising gasoline drive-by without mannequin 3.

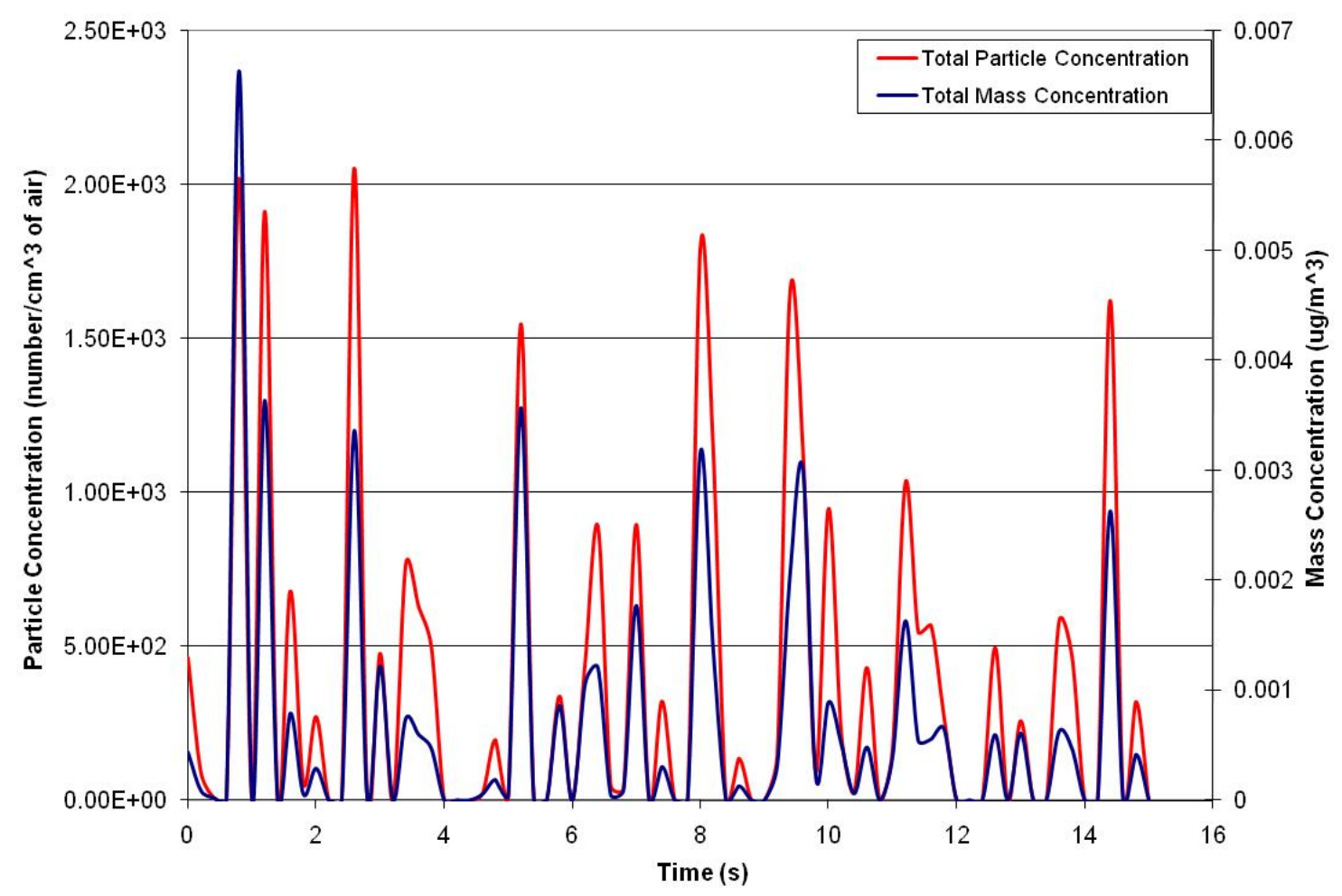

Figure 9-88: Cruising gasoline drive-by without mannequin 4. 


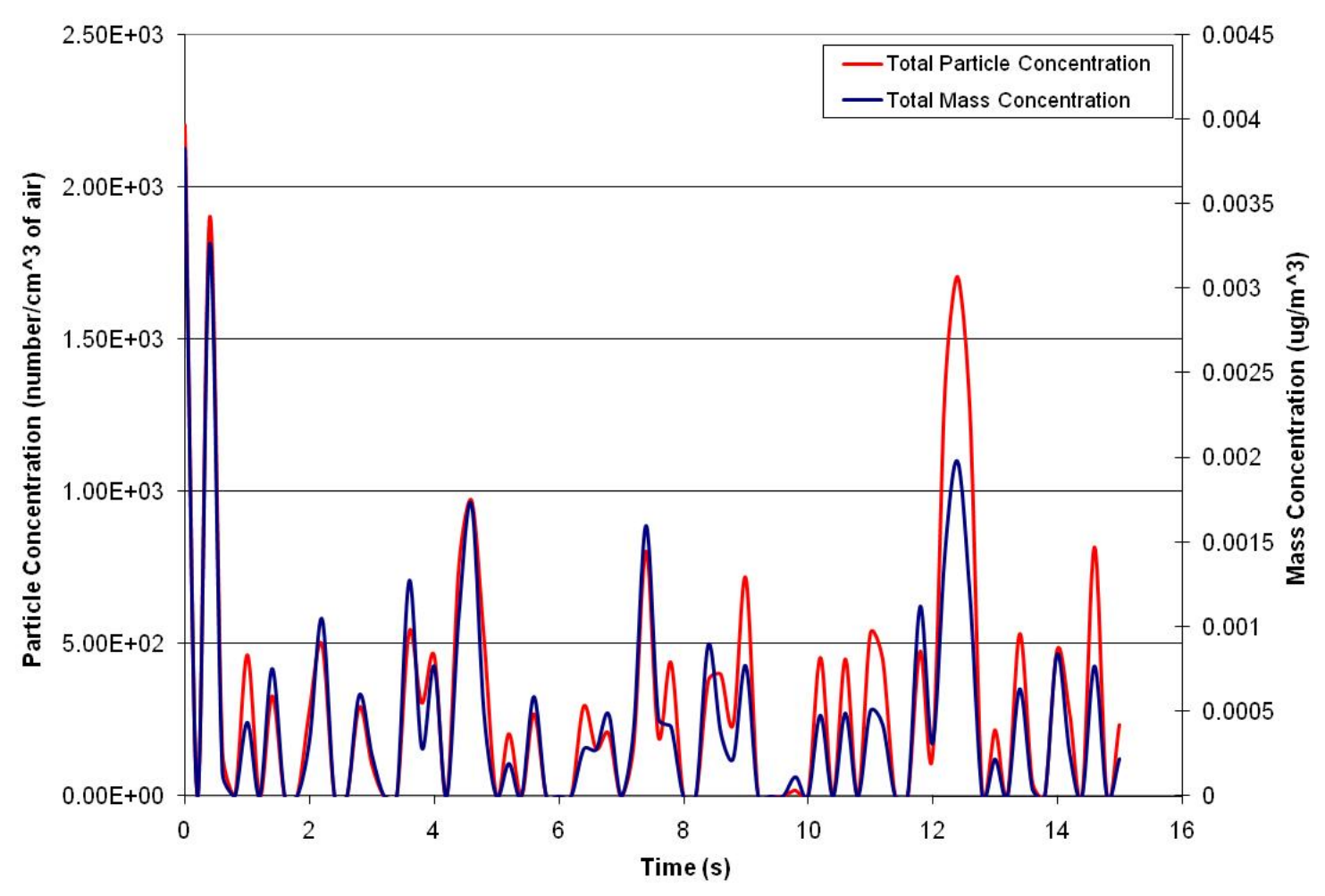

Figure 9-89: Cruising gasoline drive-by without mannequin 5.

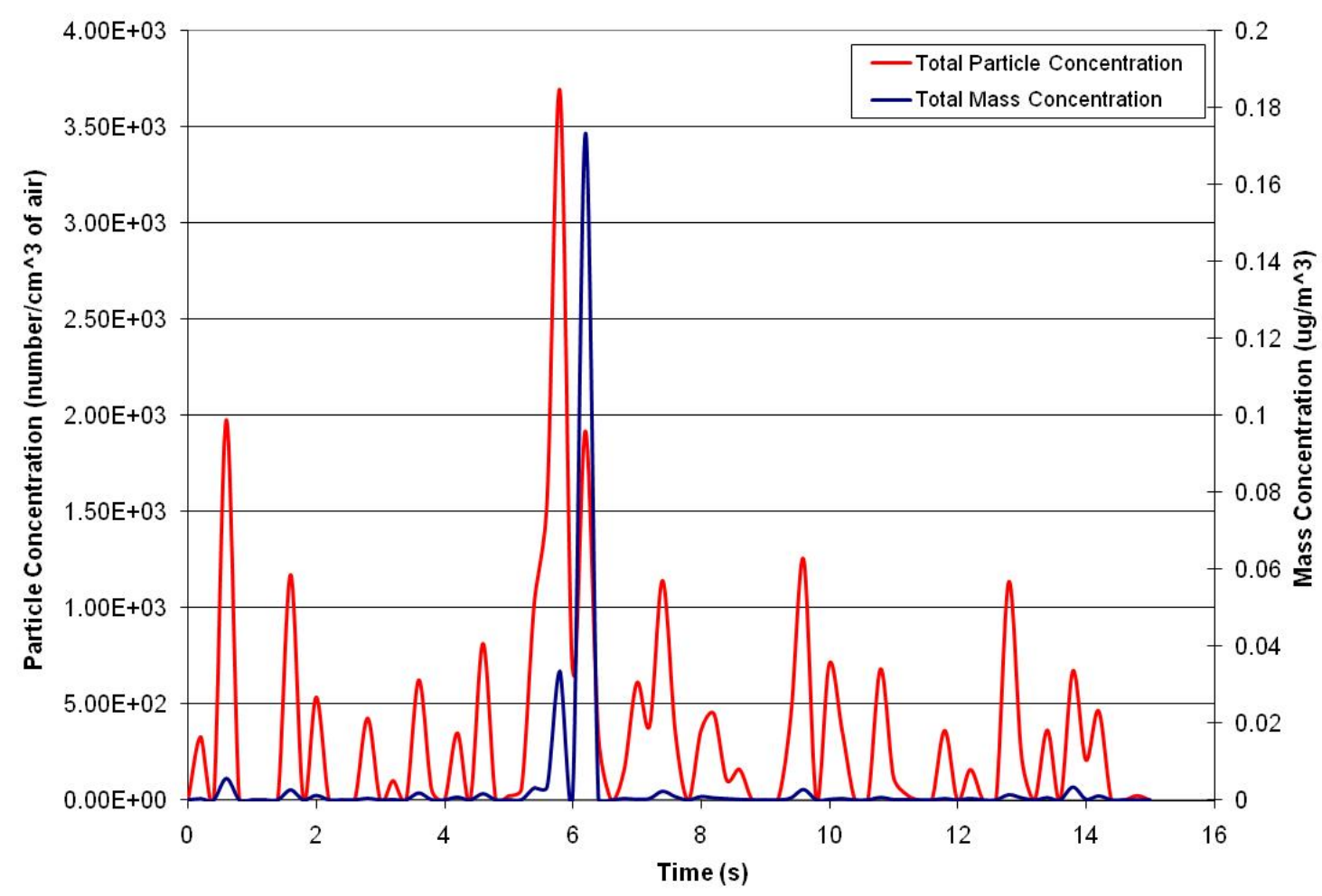

Figure 9-90: Cruising gasoline drive-by without mannequin 6. 


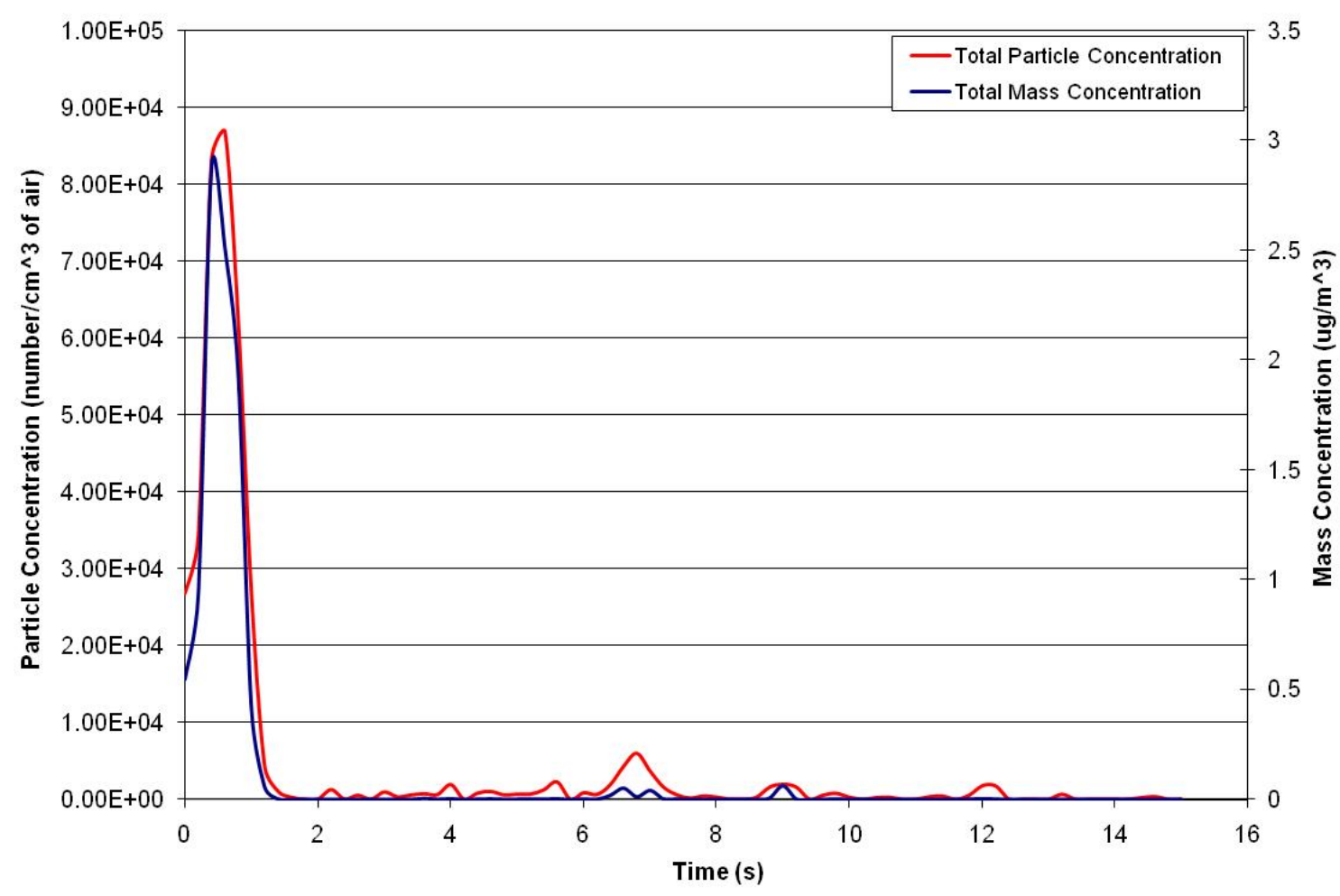

Figure 9-91: Hard acceleration gasoline drive-by with baby 1.

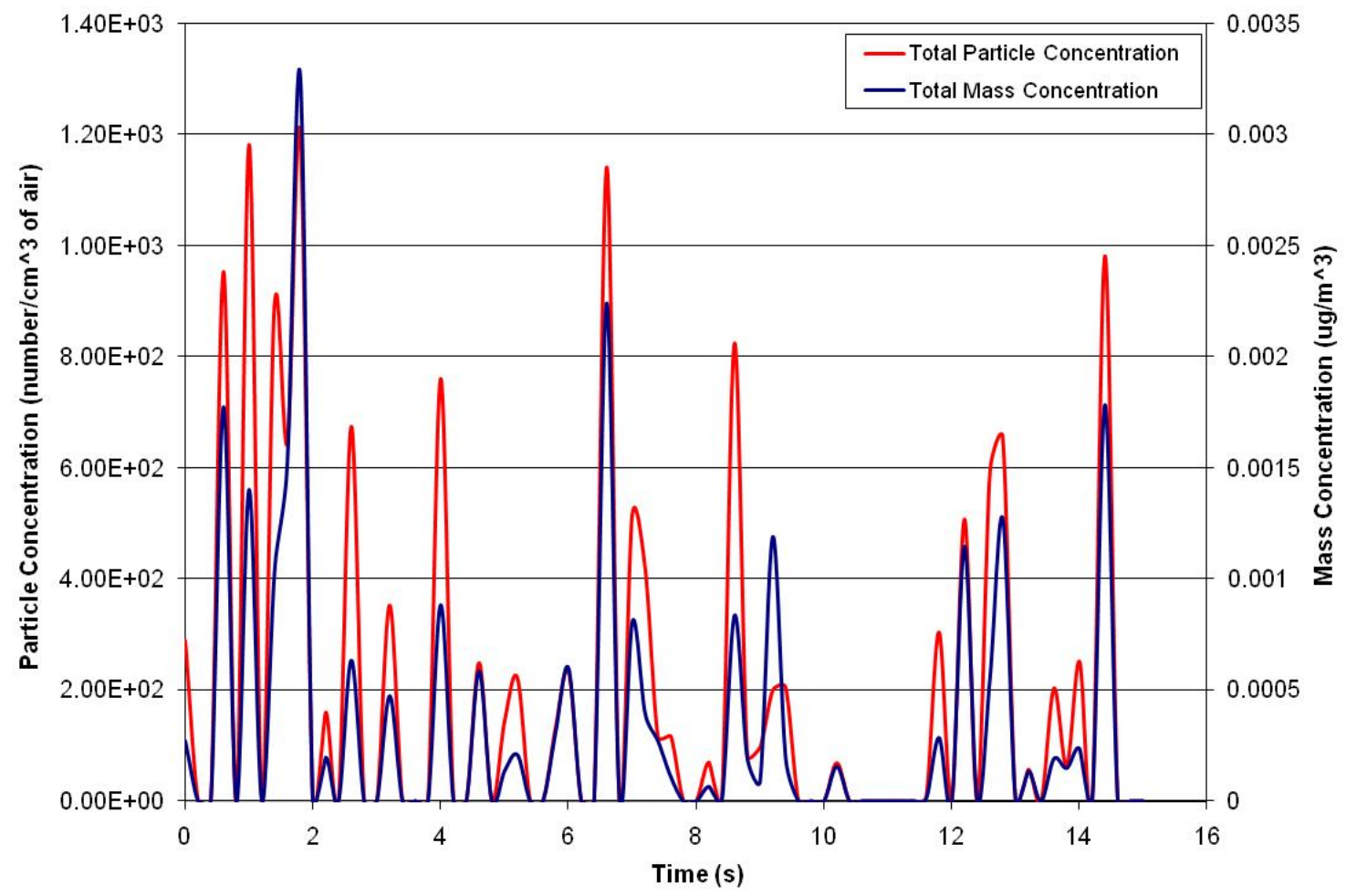

Figure 9-92: Hard acceleration gasoline drive-by with baby 2. 


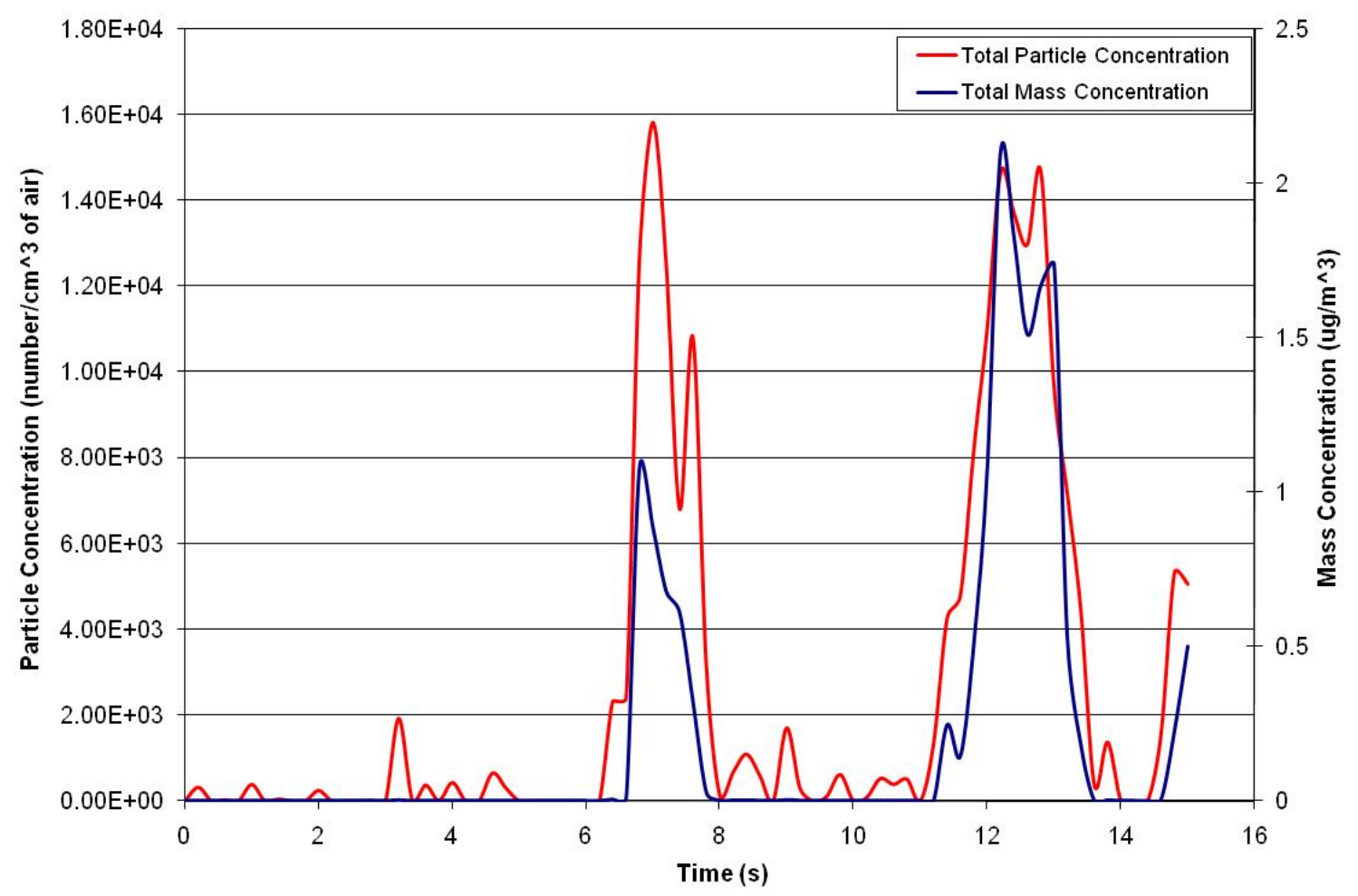

Figure 9-93: Hard acceleration gasoline drive-by with baby 3 .

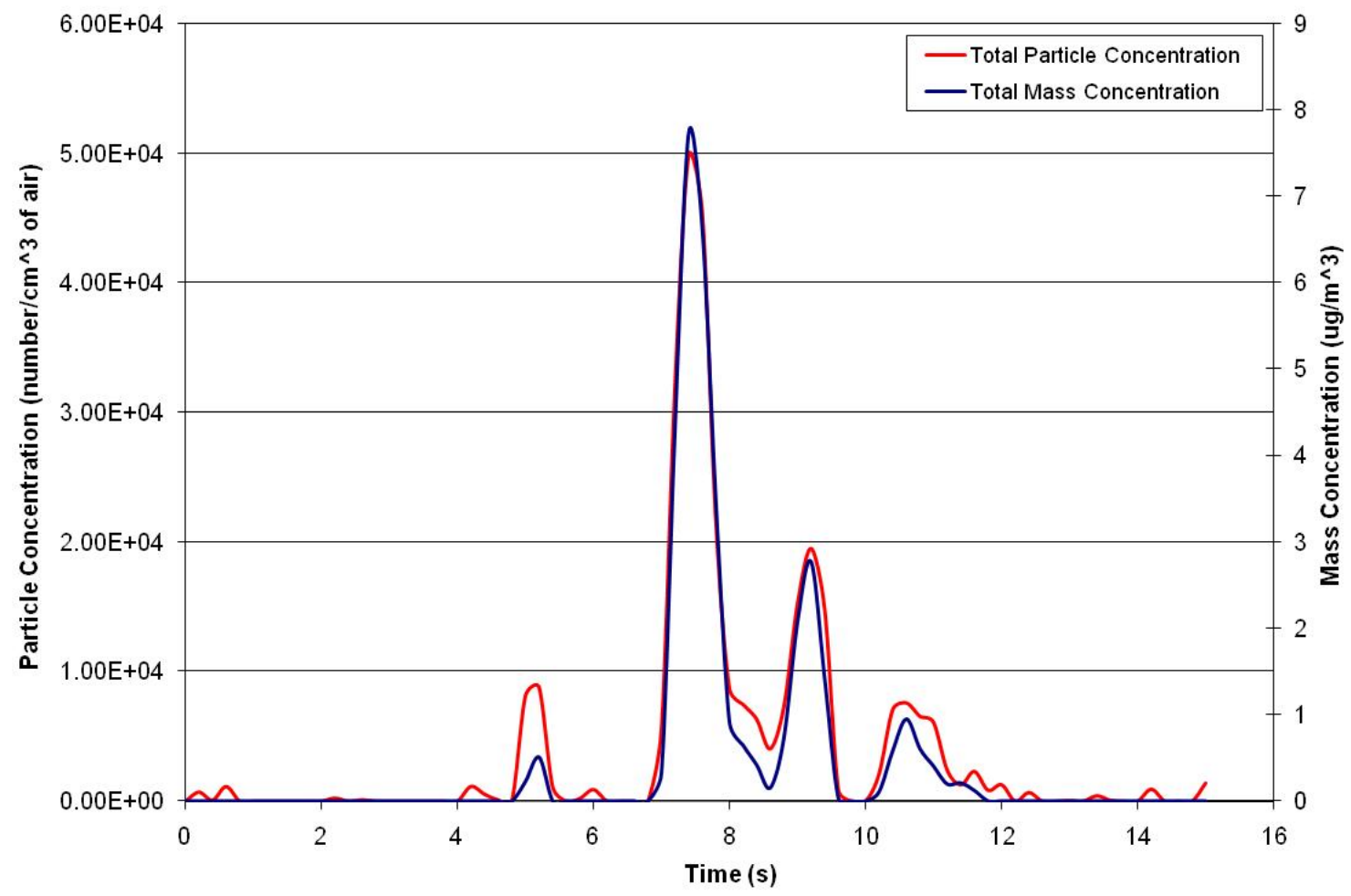

Figure 9-94: Hard acceleration gasoline drive-by with baby 4. 


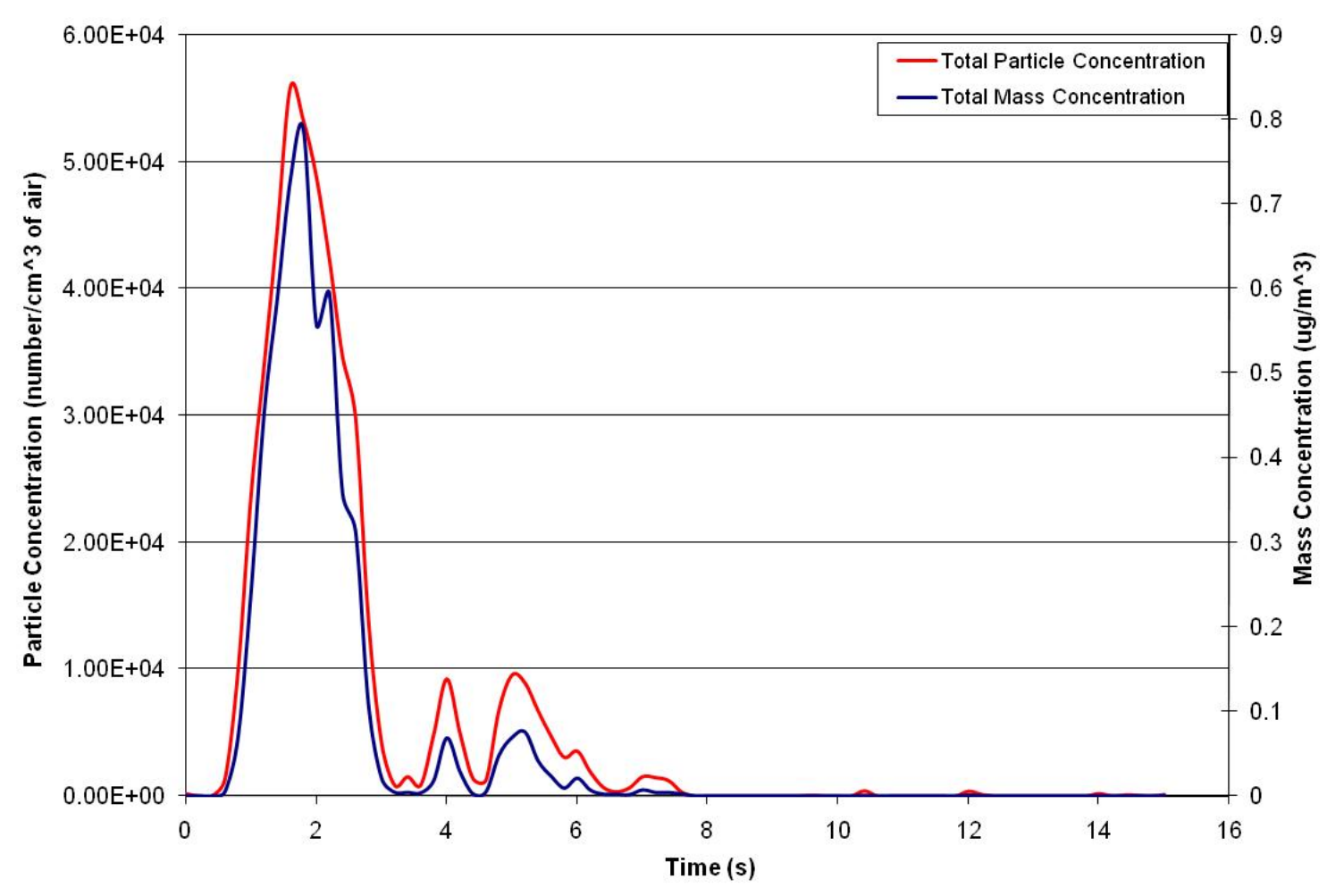

Figure 9-95: Hard acceleration gasoline drive-by with baby 5 .

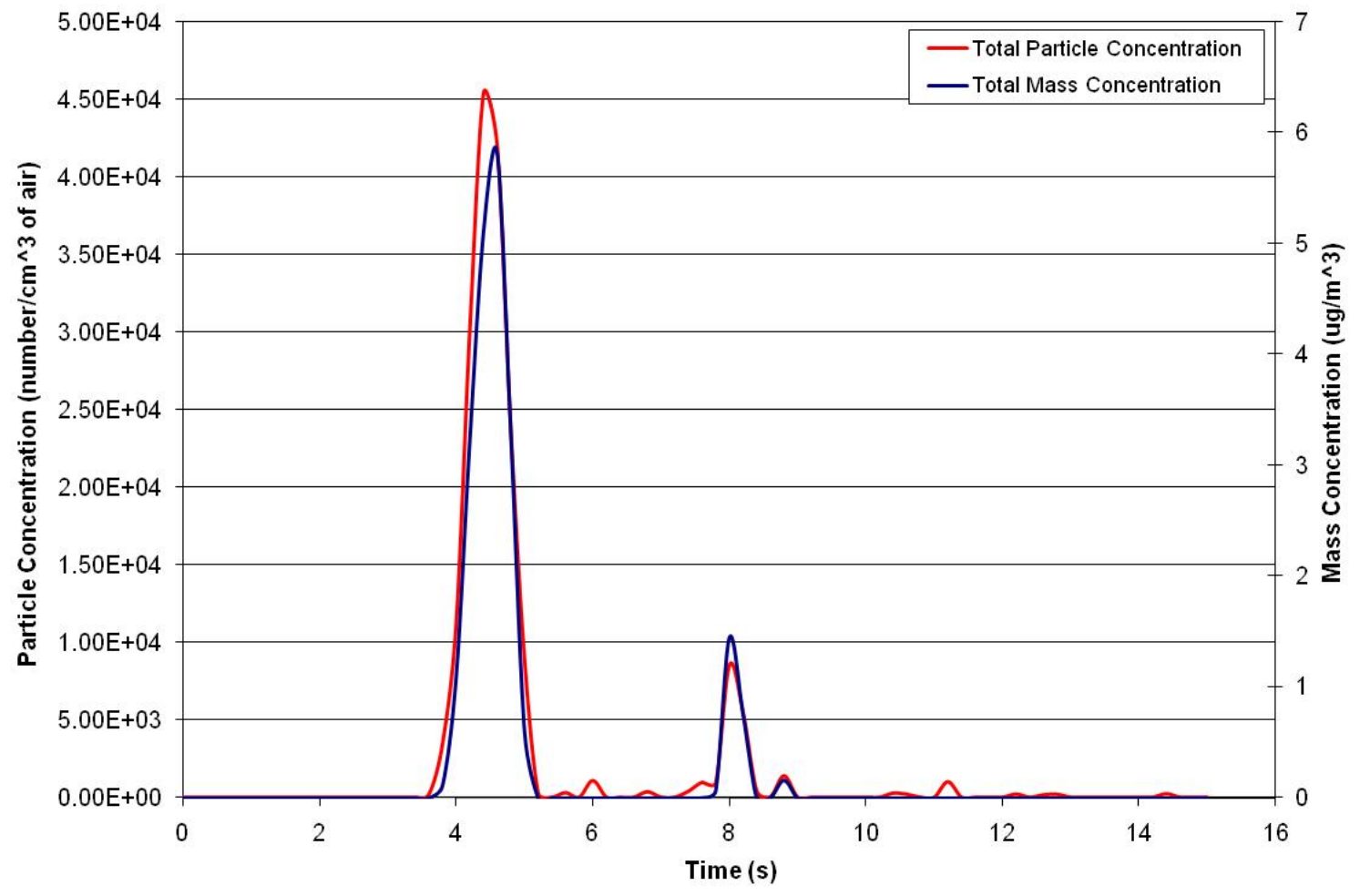

Figure 9-96: Hard acceleration gasoline drive-by with baby 6 . 


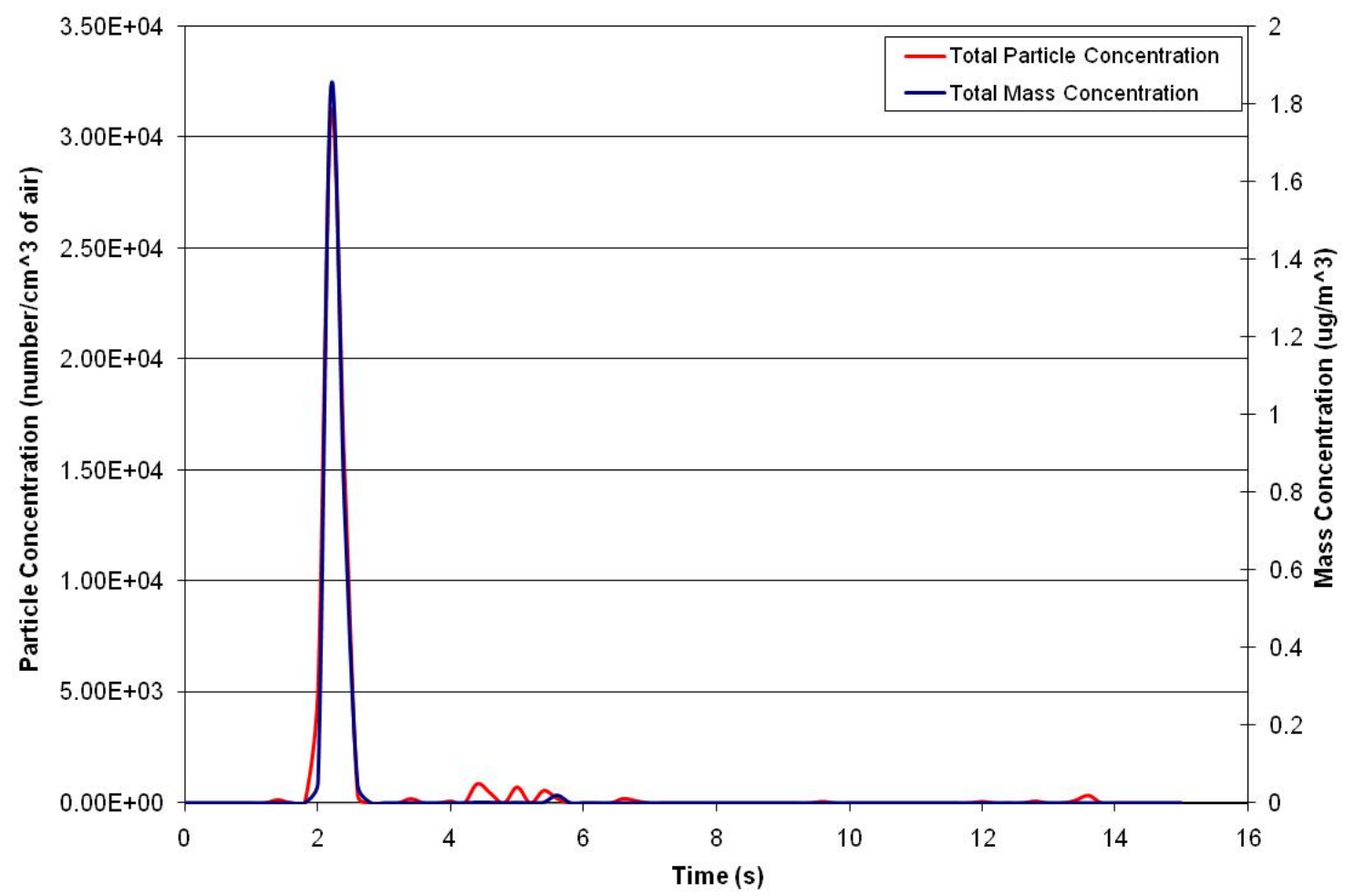

Figure 9-97: Medium acceleration gasoline drive-by with baby 1 .

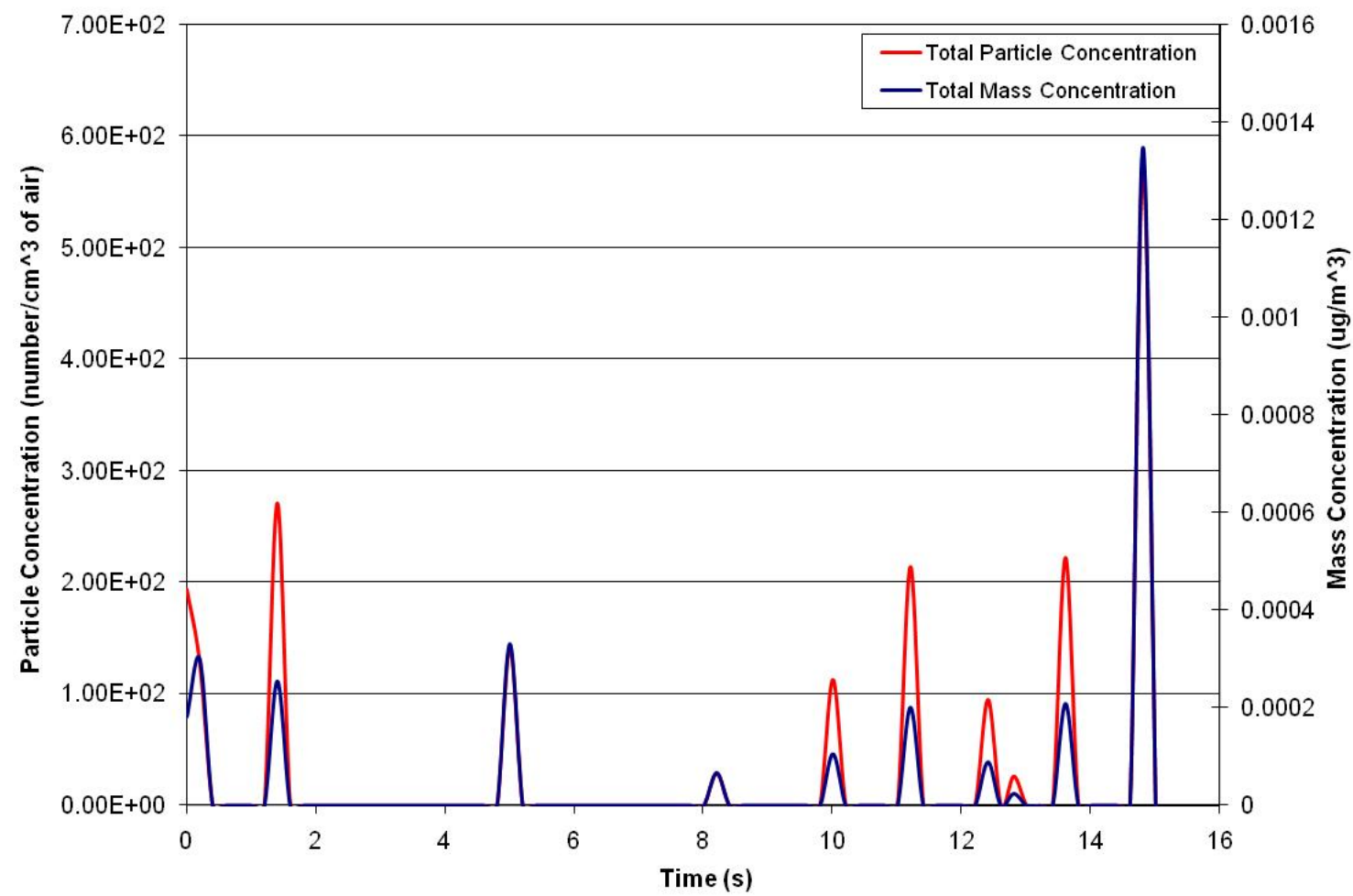

Figure 9-98: Medium acceleration gasoline drive-by with baby 2 . 


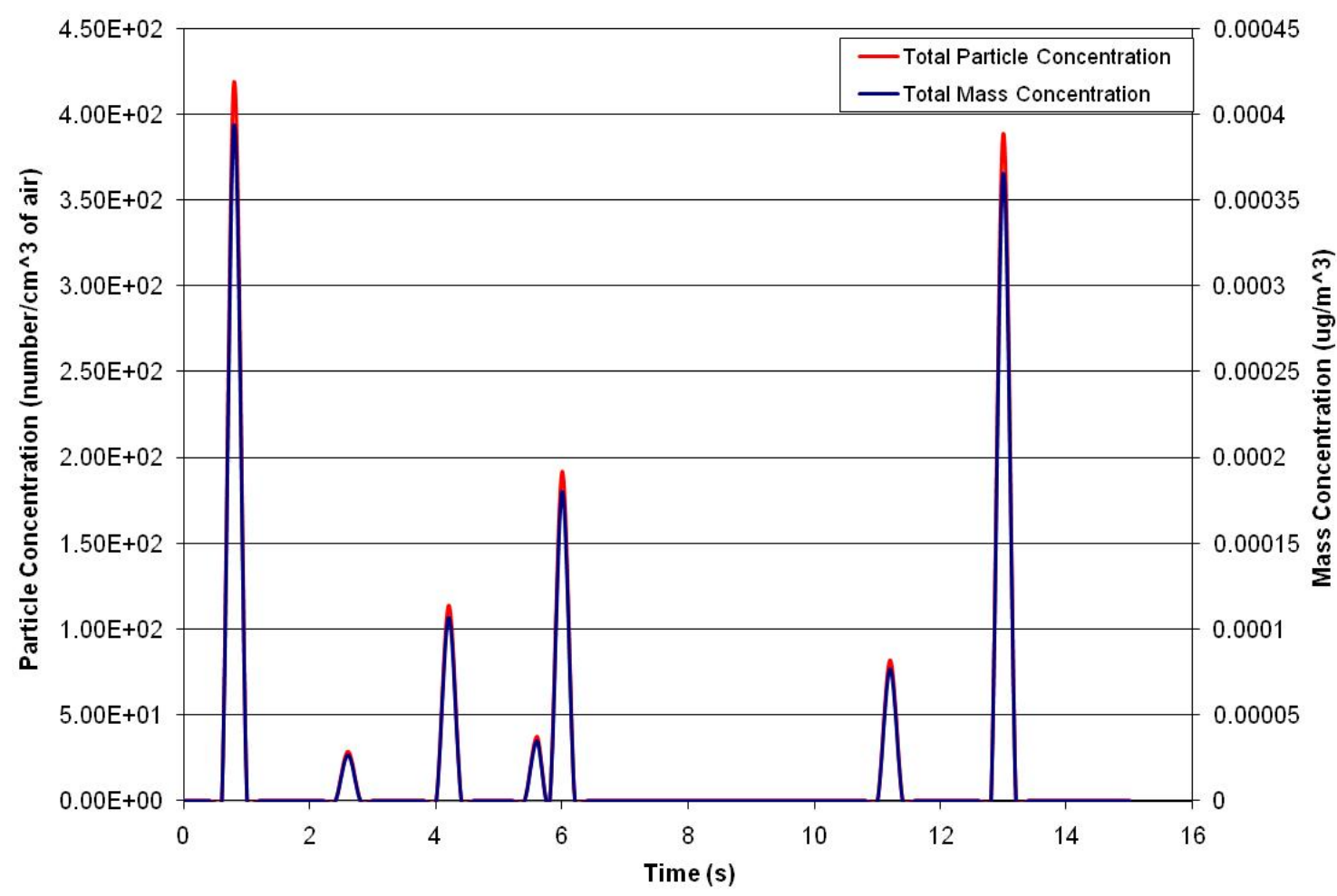

Figure 9-99: Medium acceleration gasoline drive-by with baby 3 .

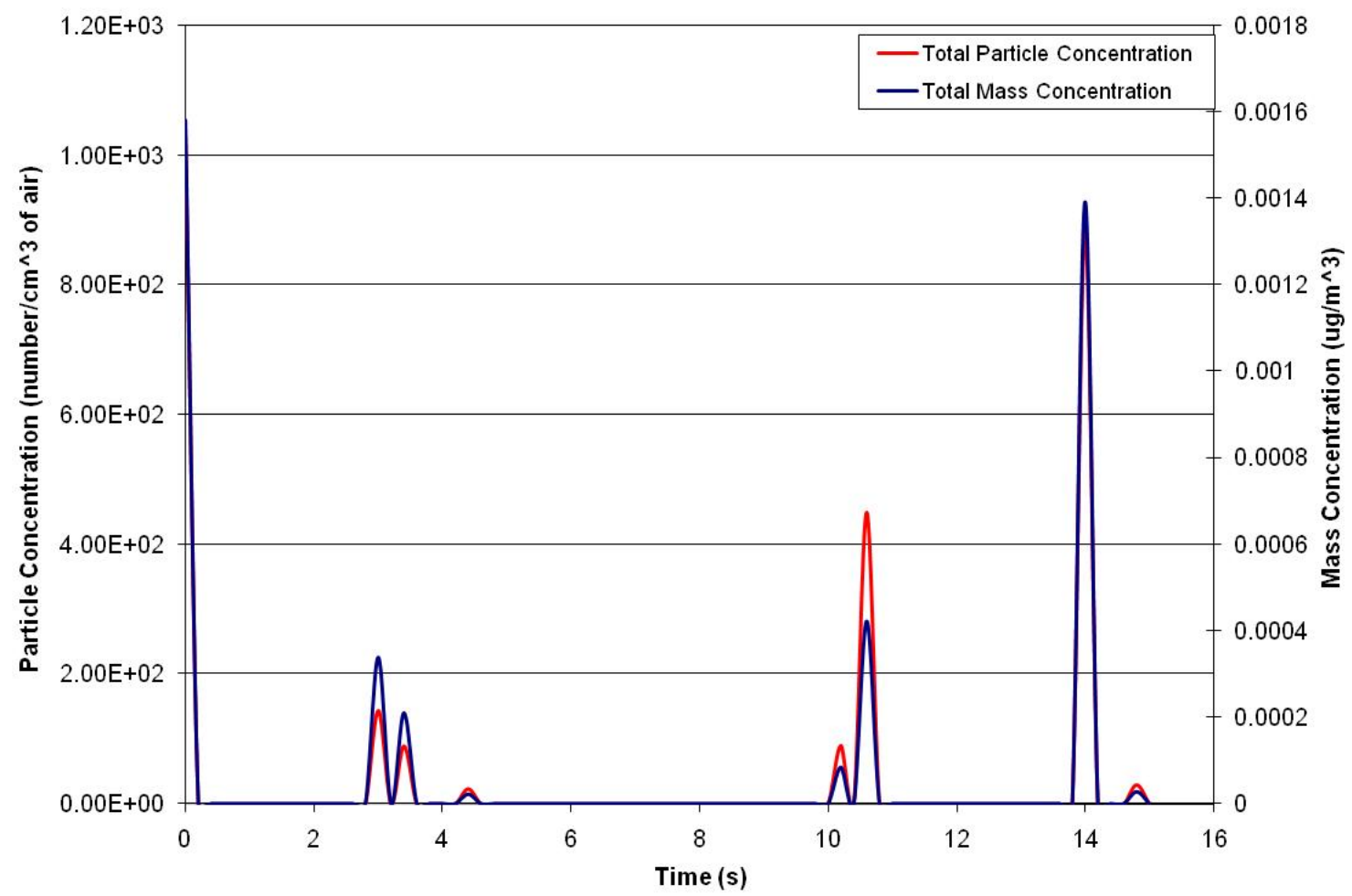

Figure 9-100: Medium acceleration gasoline drive-by with baby 4. 


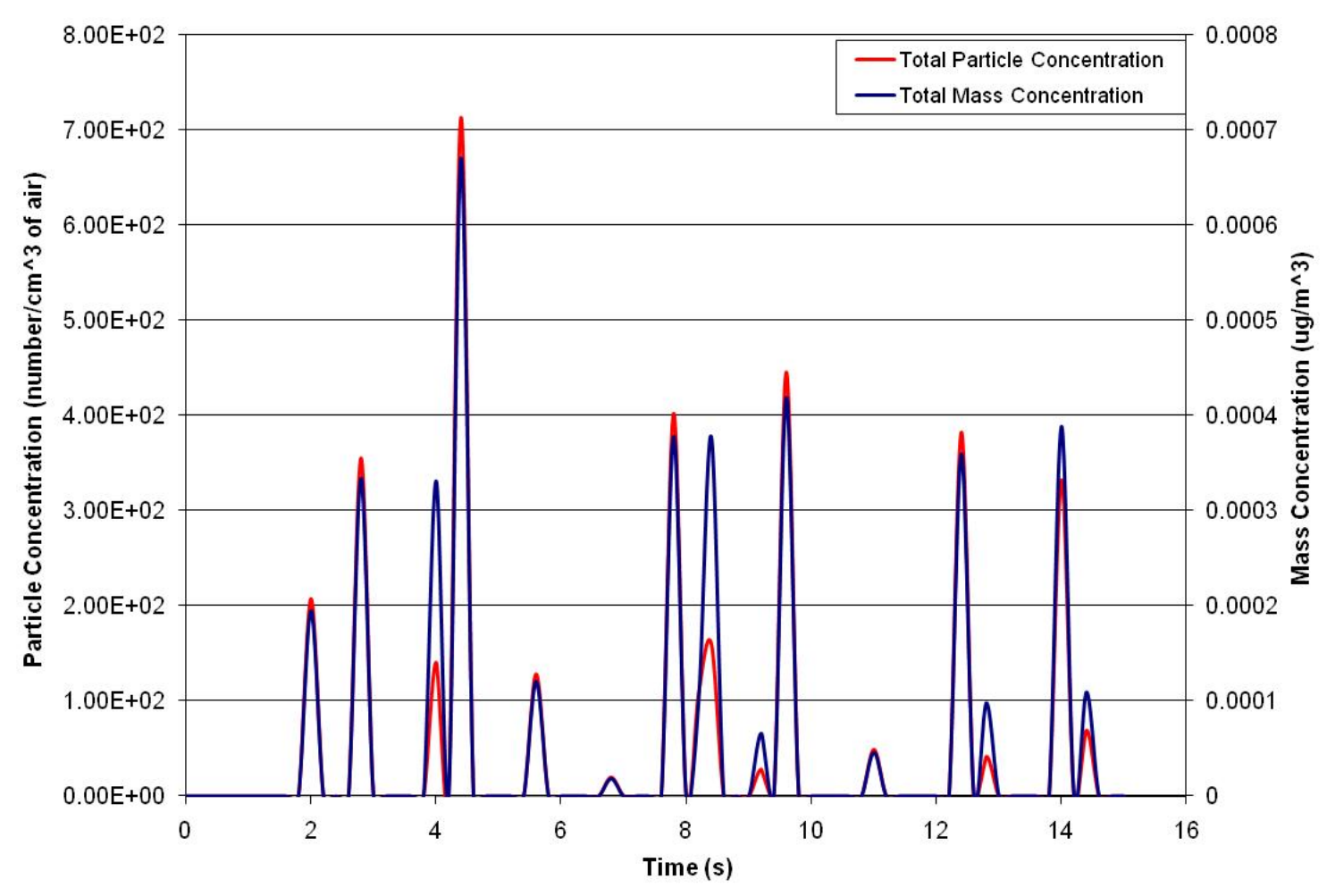

Figure 9-101: Medium acceleration gasoline drive-by with baby 5 .

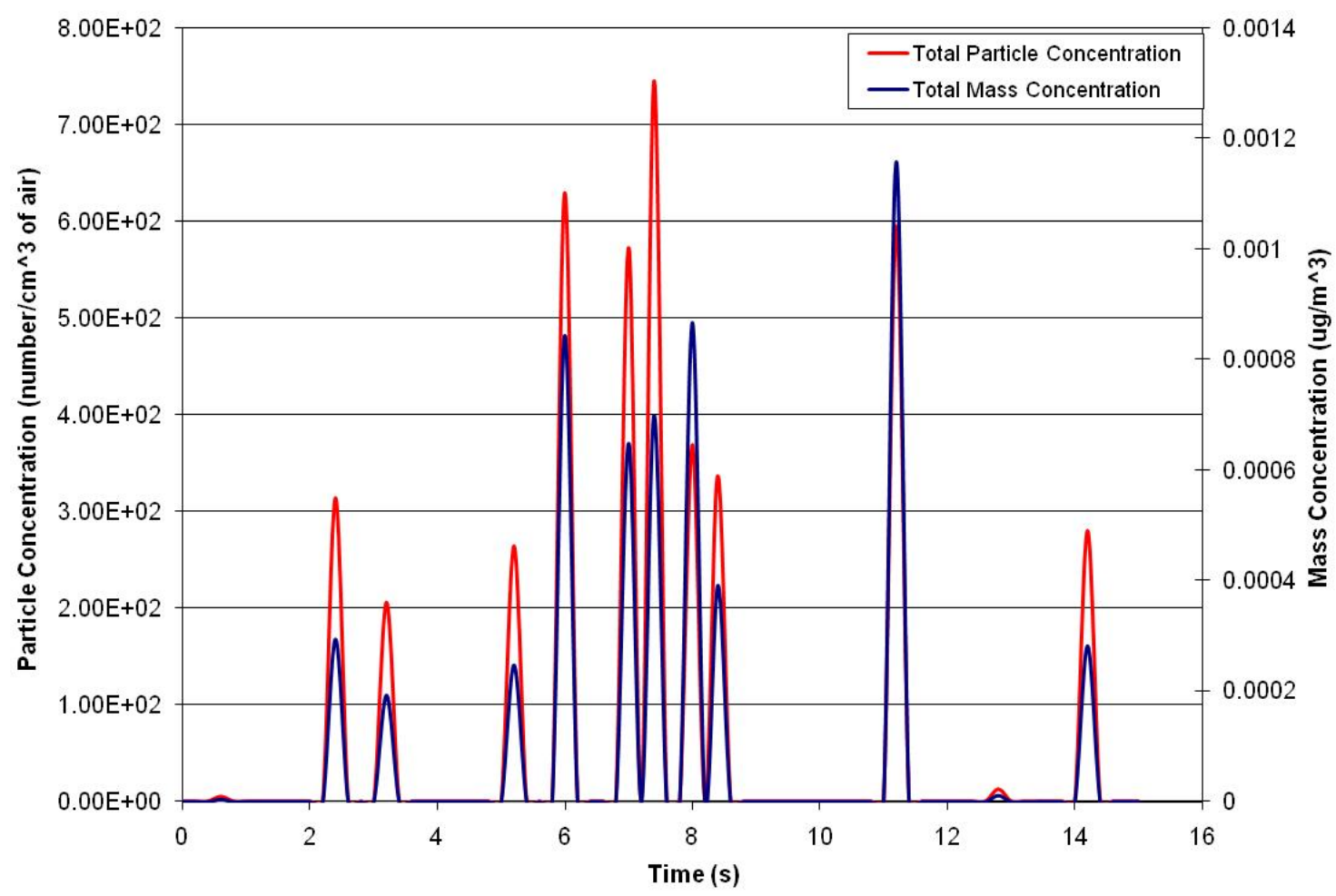

Figure 9-102: Medium acceleration gasoline drive-by with baby 6 . 


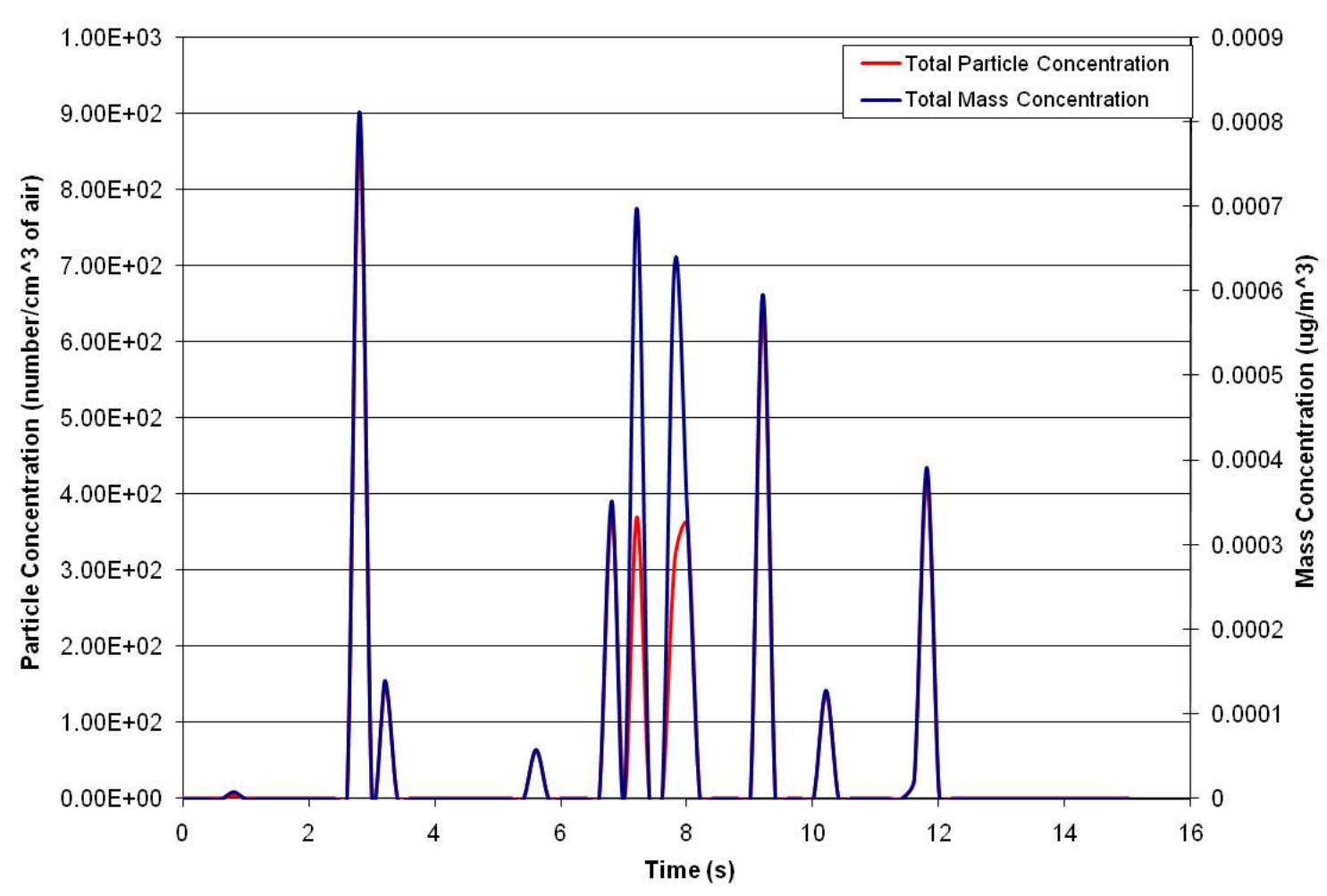

Figure 9-103: Cruising gasoline drive-by with baby 1 .

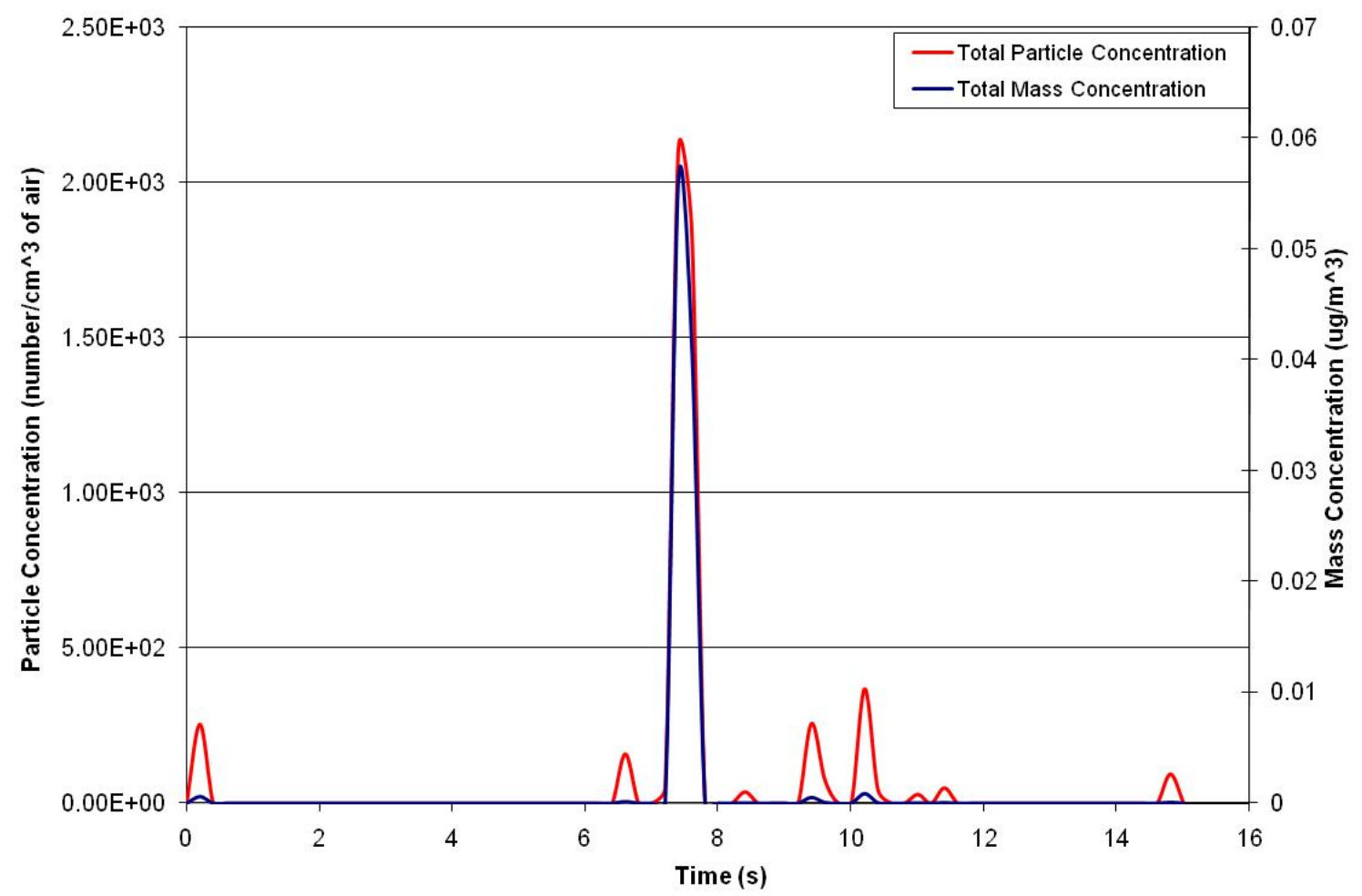

Figure 9-104: Cruising gasoline drive-by with baby 2 . 


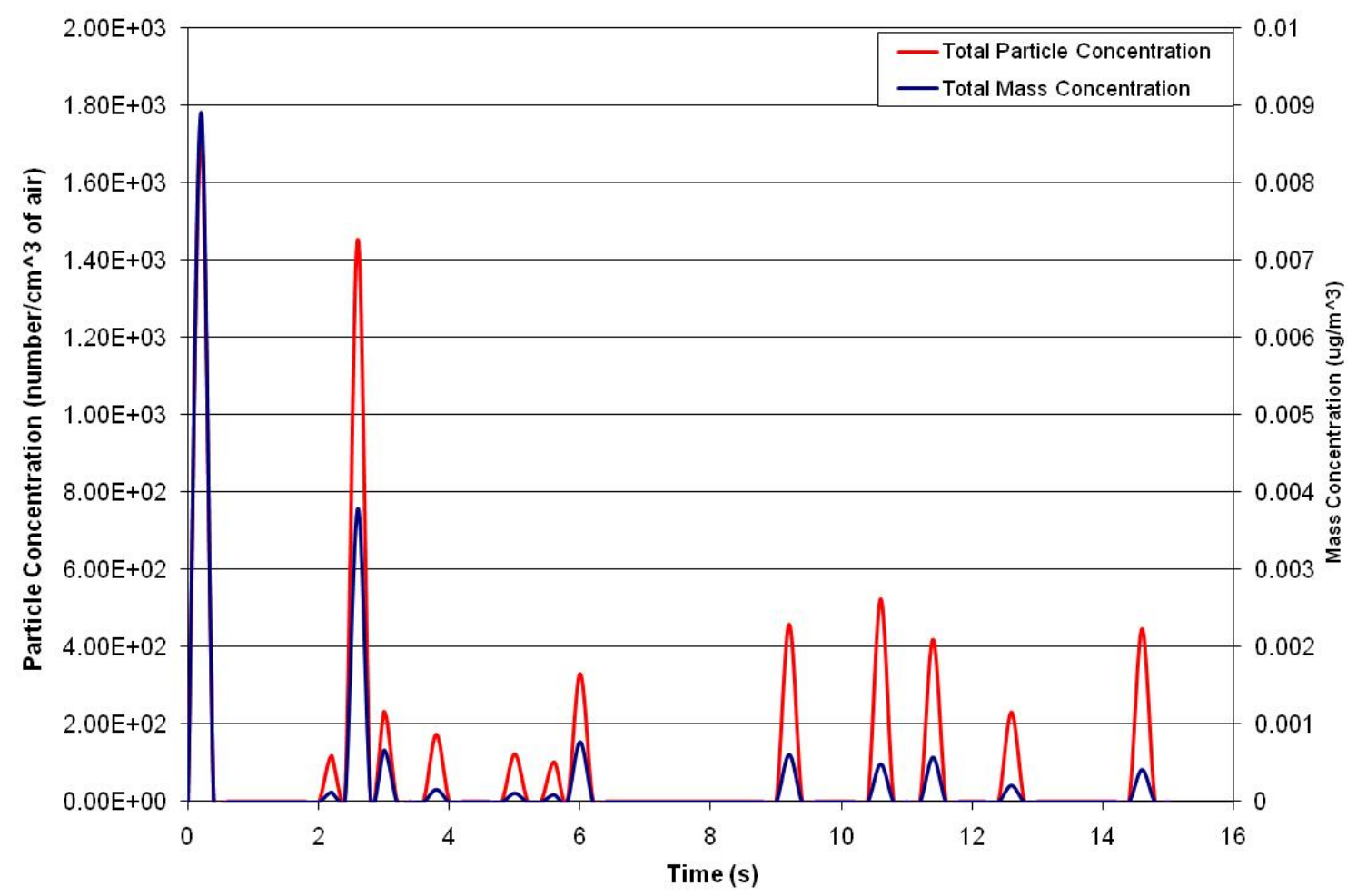

Figure 9-105: Cruising gasoline drive-by with baby 3 .

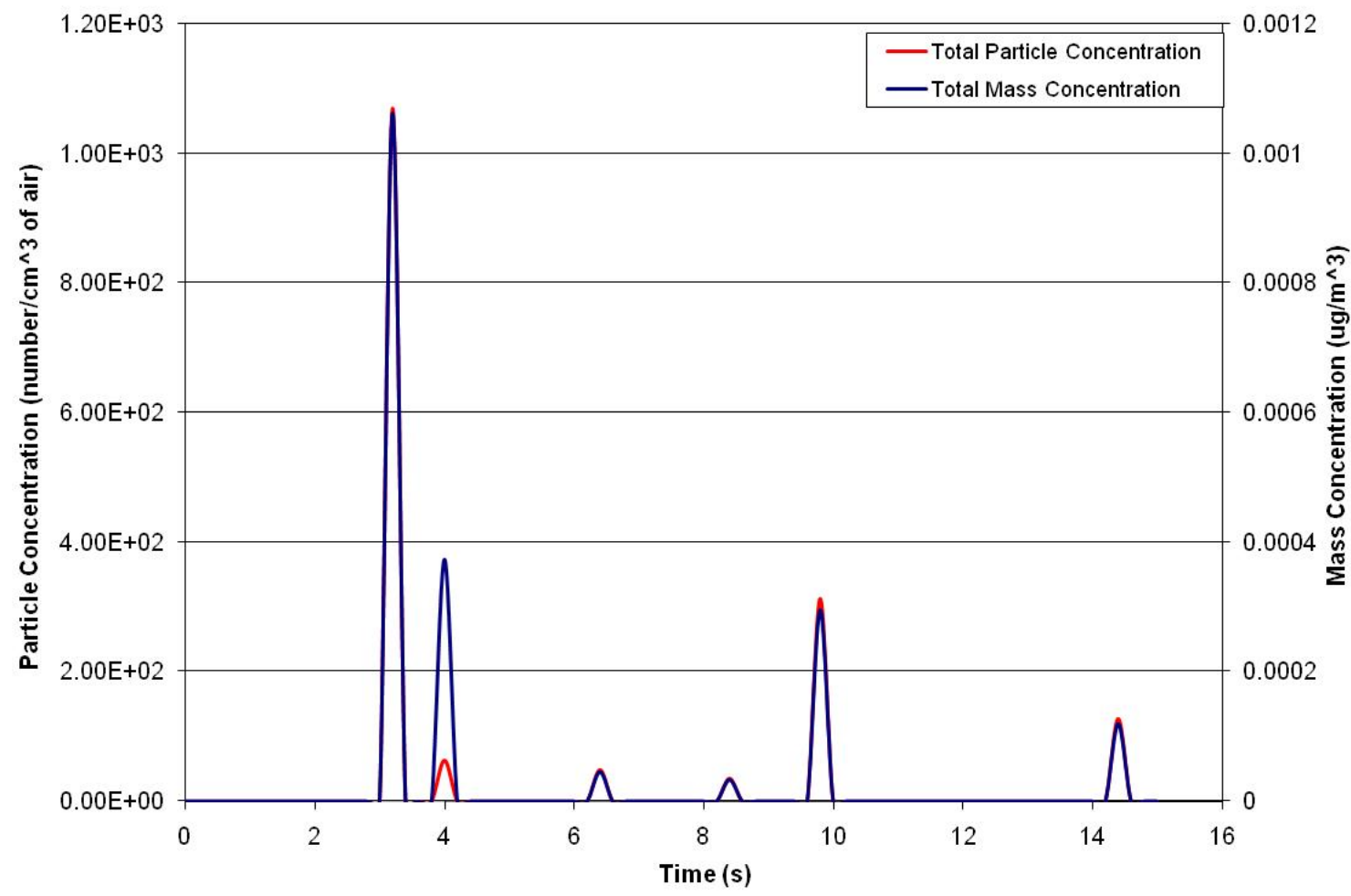

Figure 9-106: Cruising gasoline drive-by with baby 4 . 


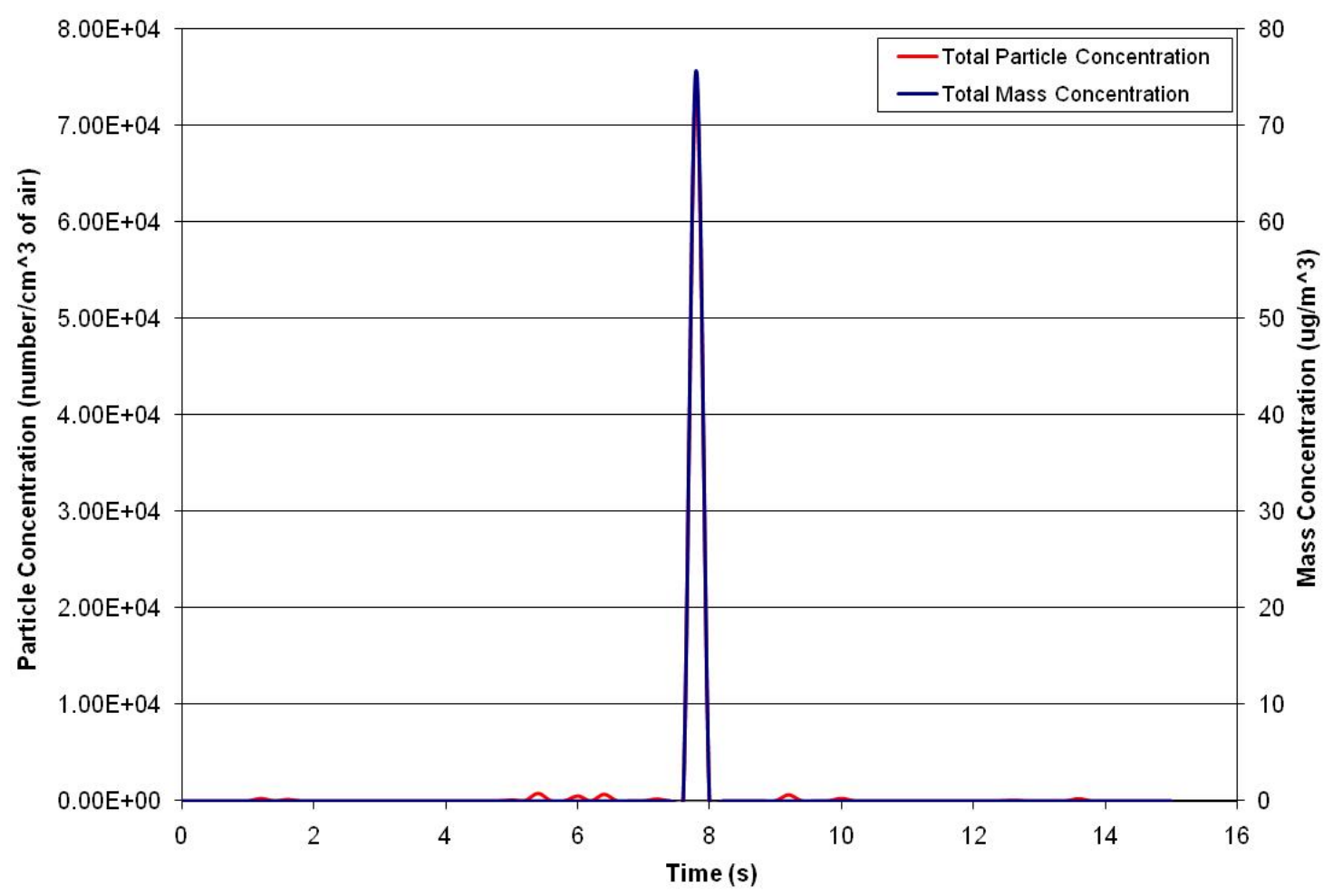

Figure 9-107: Cruising gasoline drive-by with baby 5 .

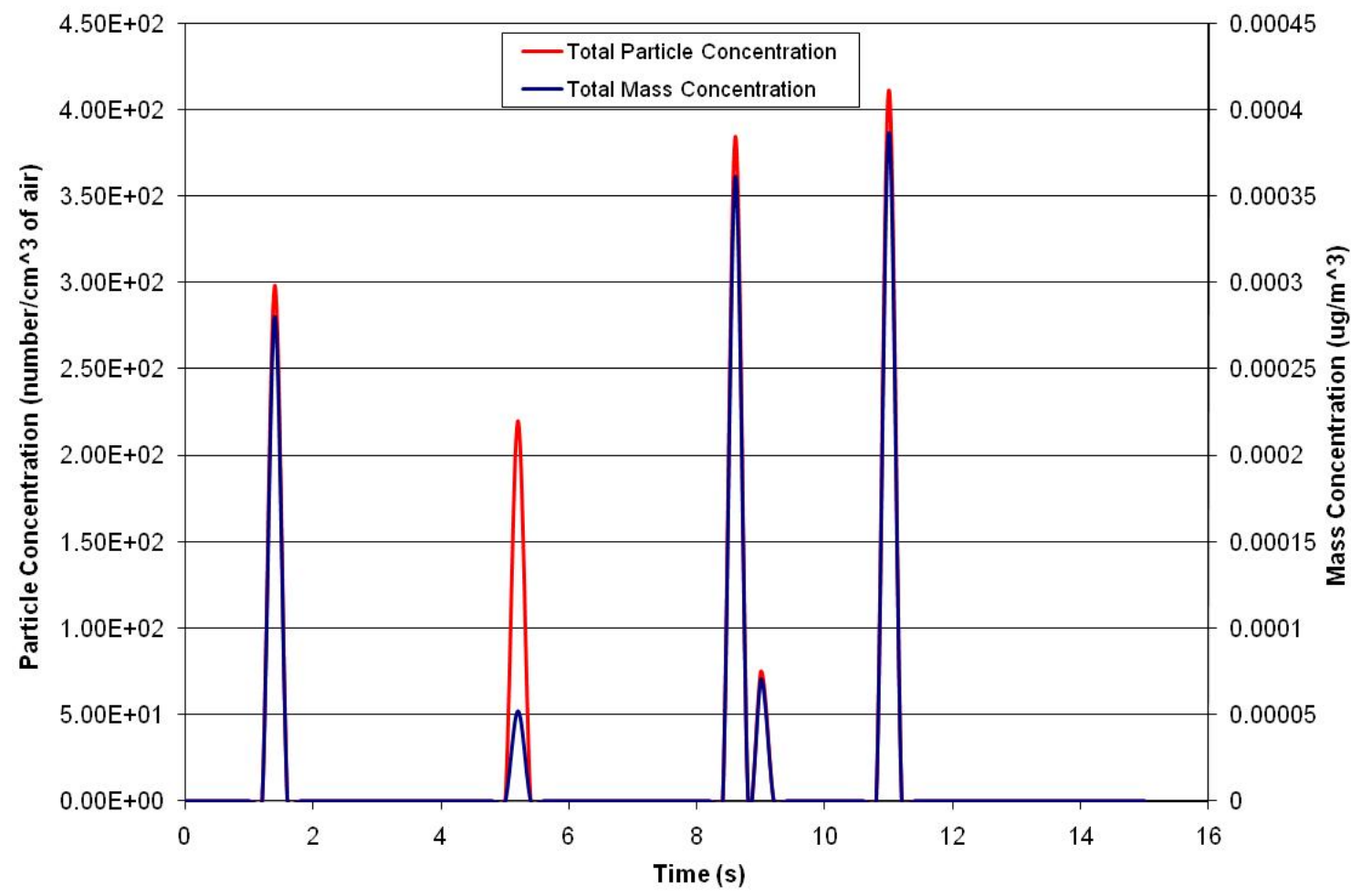

Figure 9-108: Cruising gasoline drive-by with baby 6 . 


\section{Appendix D: Particle size distributions}

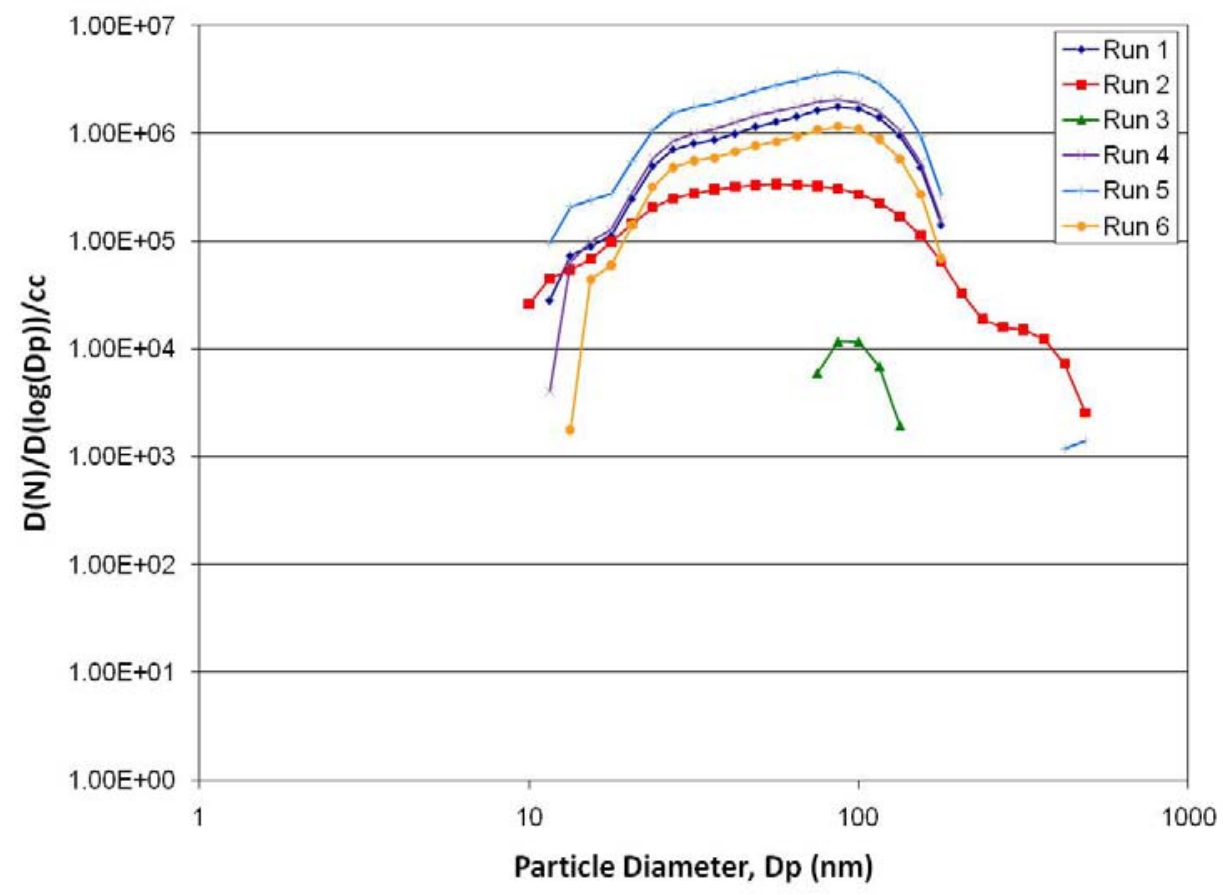

Figure 10-1: Lognormal particle size distribution for hard acceleration diesel vehicle tests with mannequin.

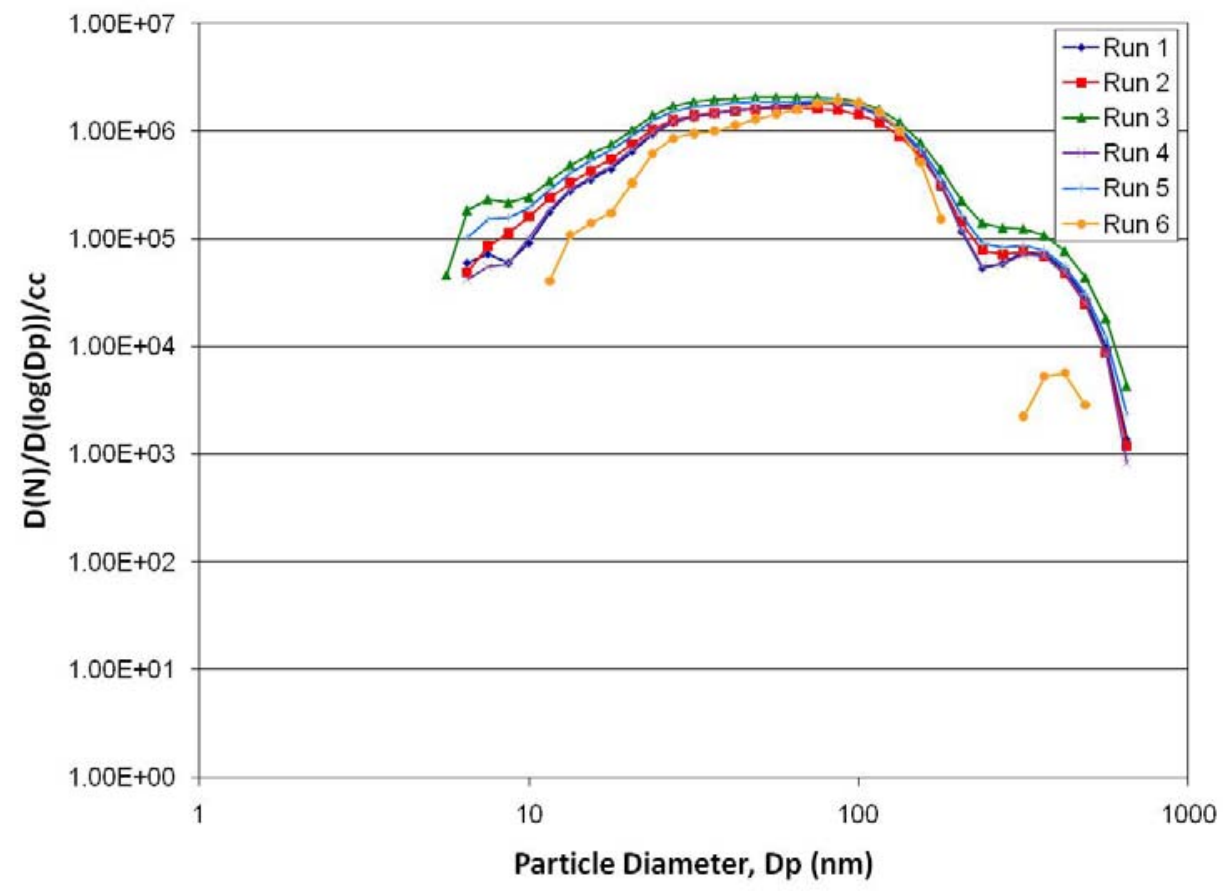

Figure 10-2: Lognormal particle size distribution for hard acceleration diesel vehicle tests with stroller.

D1 


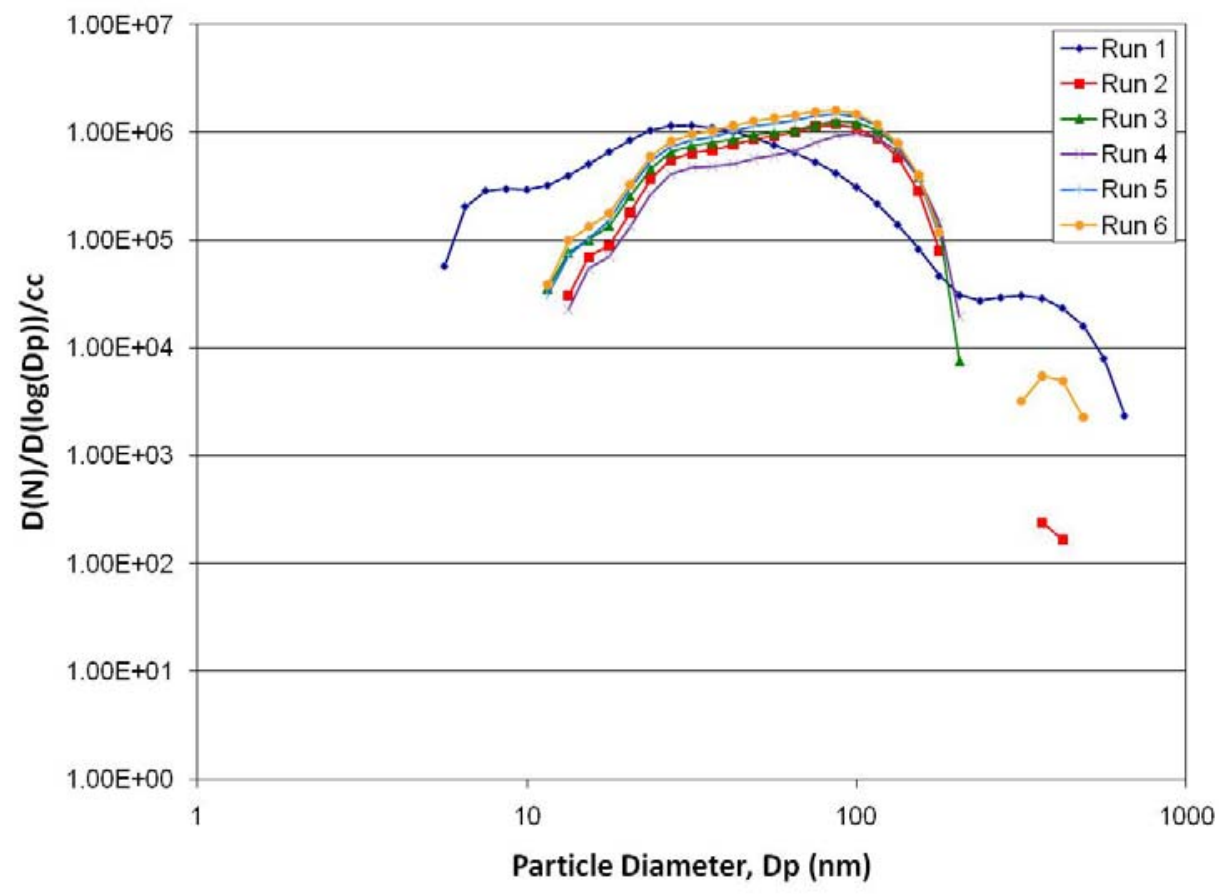

Figure 10-3: Lognormal particle size distribution for hard acceleration diesel vehicle tests without mannequin.

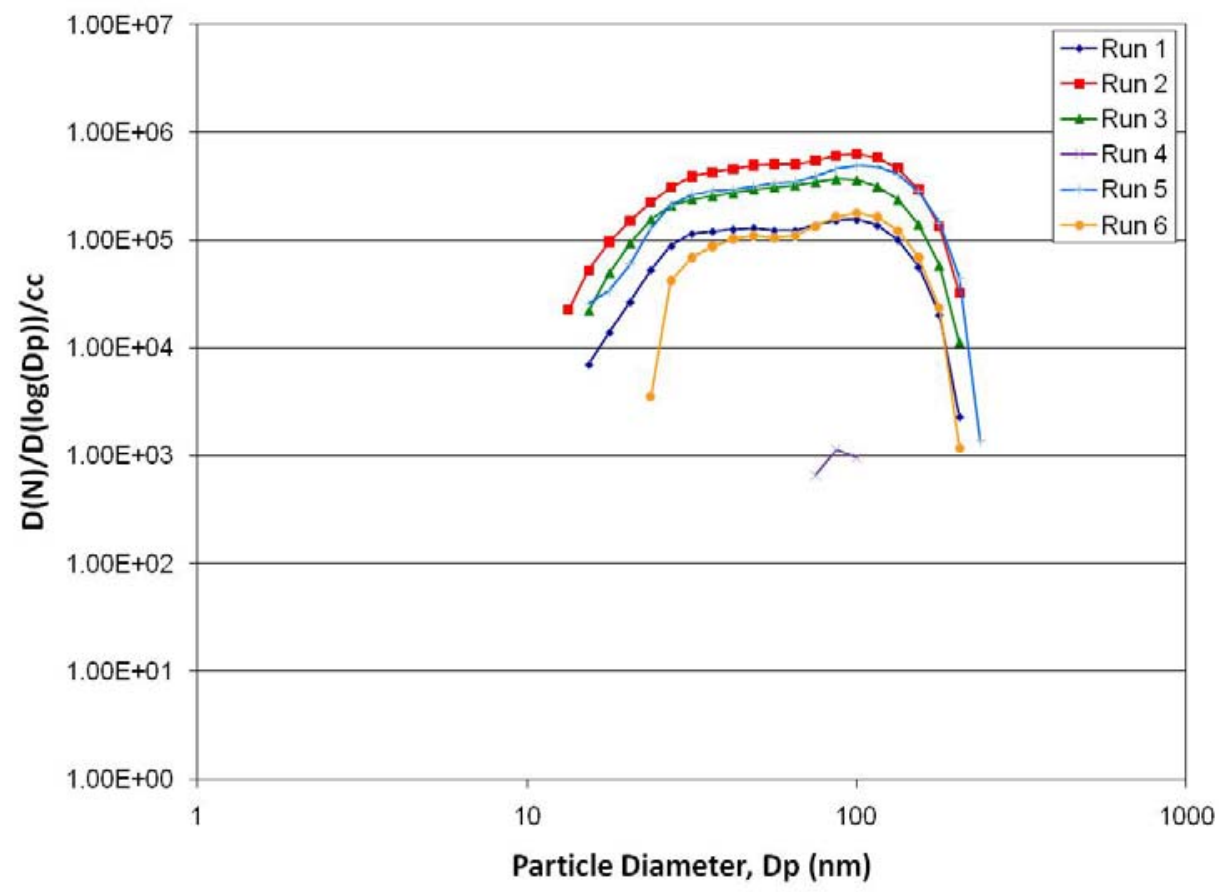

Figure 10-4: Lognormal particle size distribution for medium acceleration diesel vehicle tests with mannequin. 


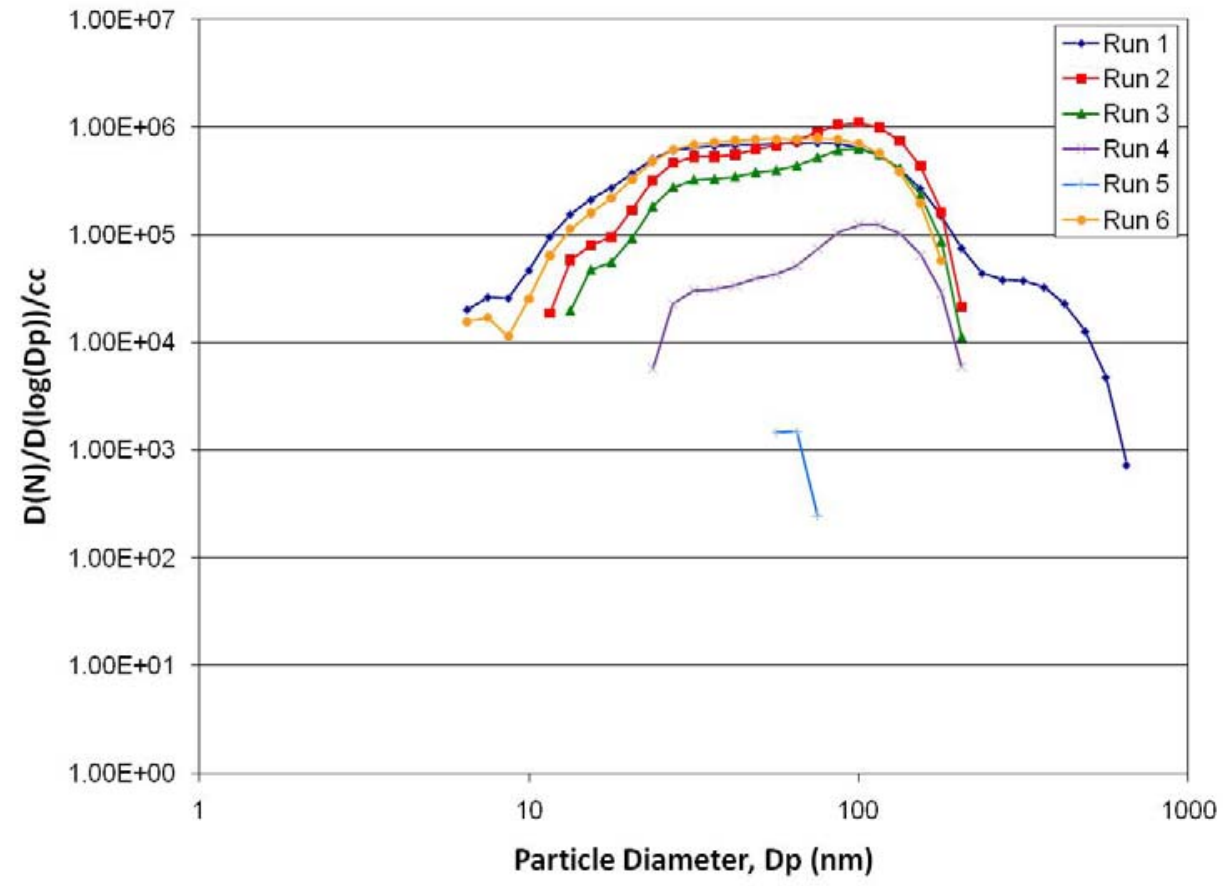

Figure 10-5: Lognormal particle size distribution for medium acceleration diesel vehicle tests with stroller.

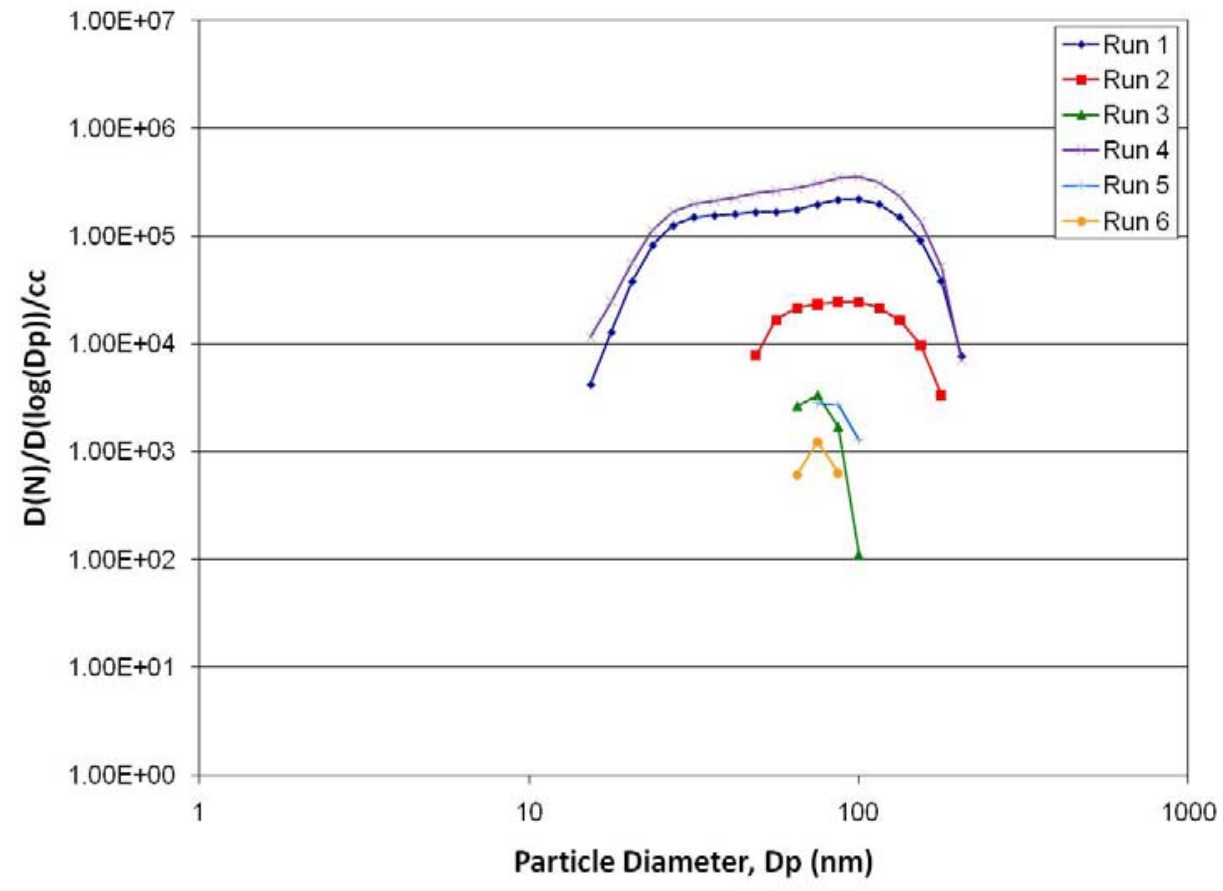

Figure 10-6: Lognormal particle size distribution for medium acceleration diesel vehicle tests without mannequin. 


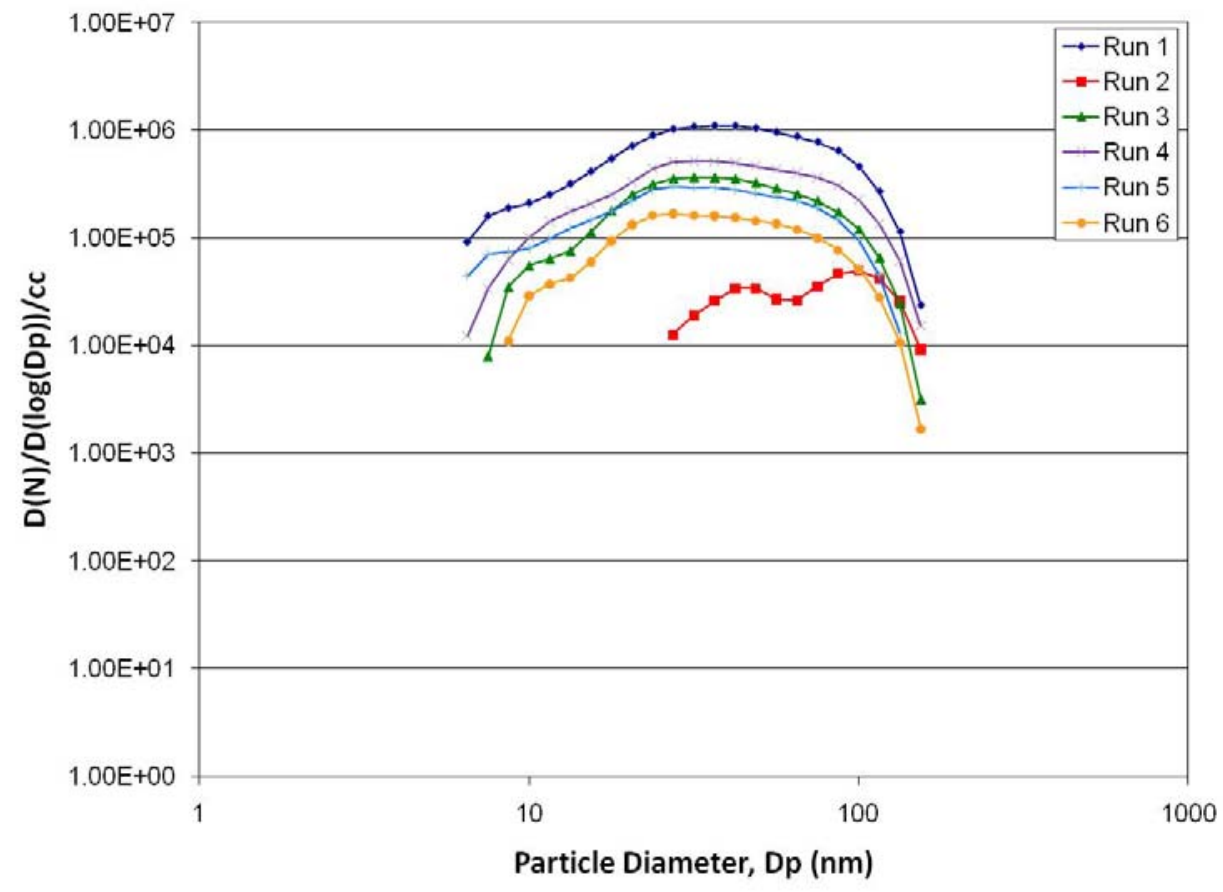

Figure 10-7: Lognormal particle size distribution for cruising diesel vehicle tests with mannequin.

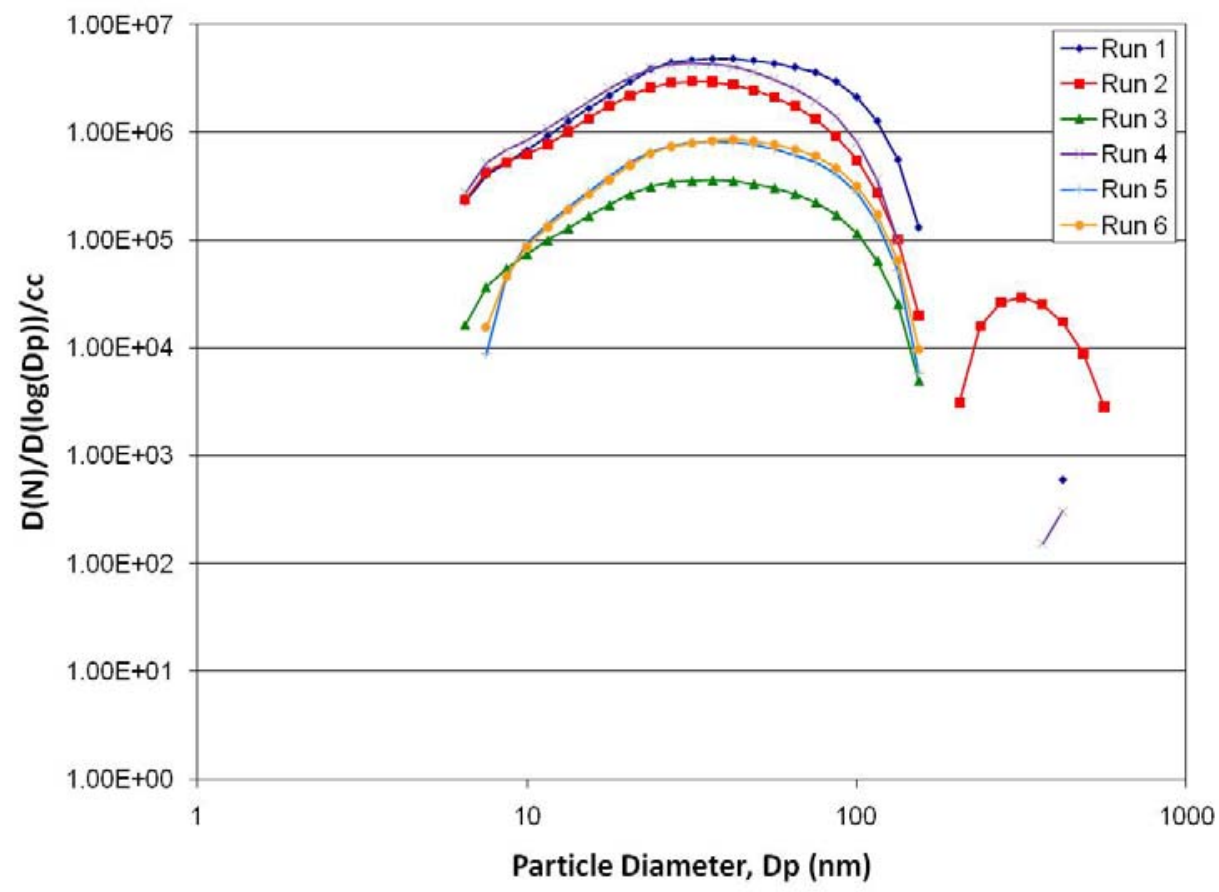

Figure 10-8: Lognormal particle size distribution for cruising diesel vehicle tests with stroller. 


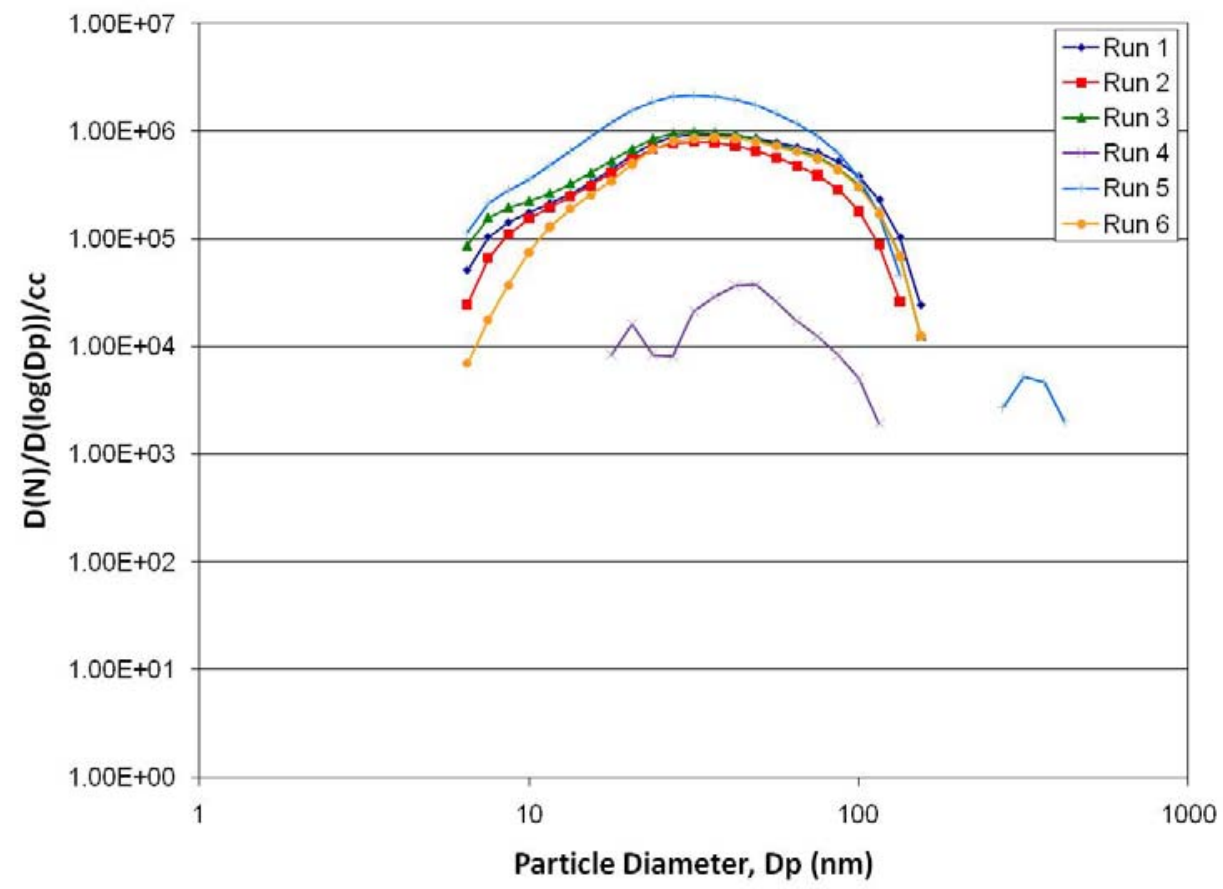

Figure 10-9: Lognormal particle size distribution for cruising diesel vehicle tests without mannequin.

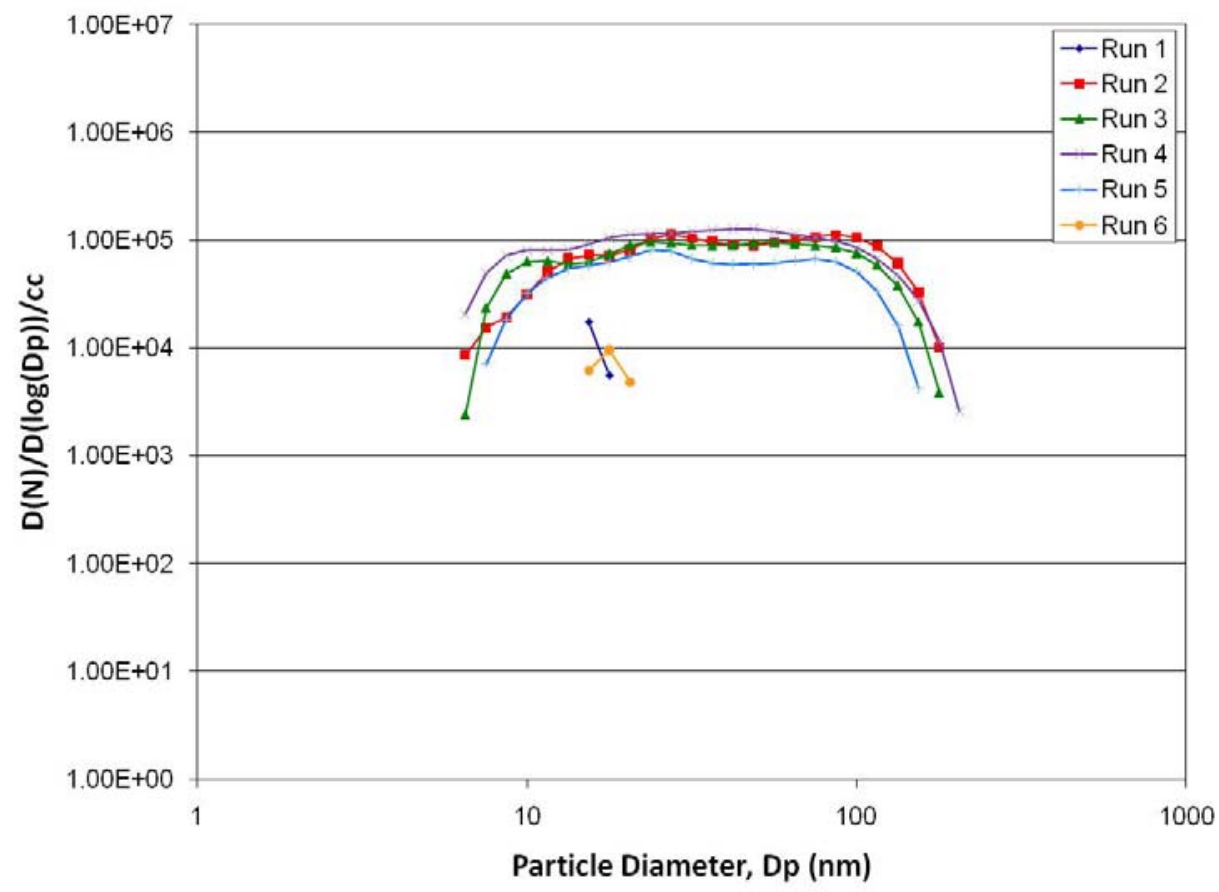

Figure 10-10: Lognormal particle size distribution for hard acceleration gasoline vehicle tests with mannequin. 


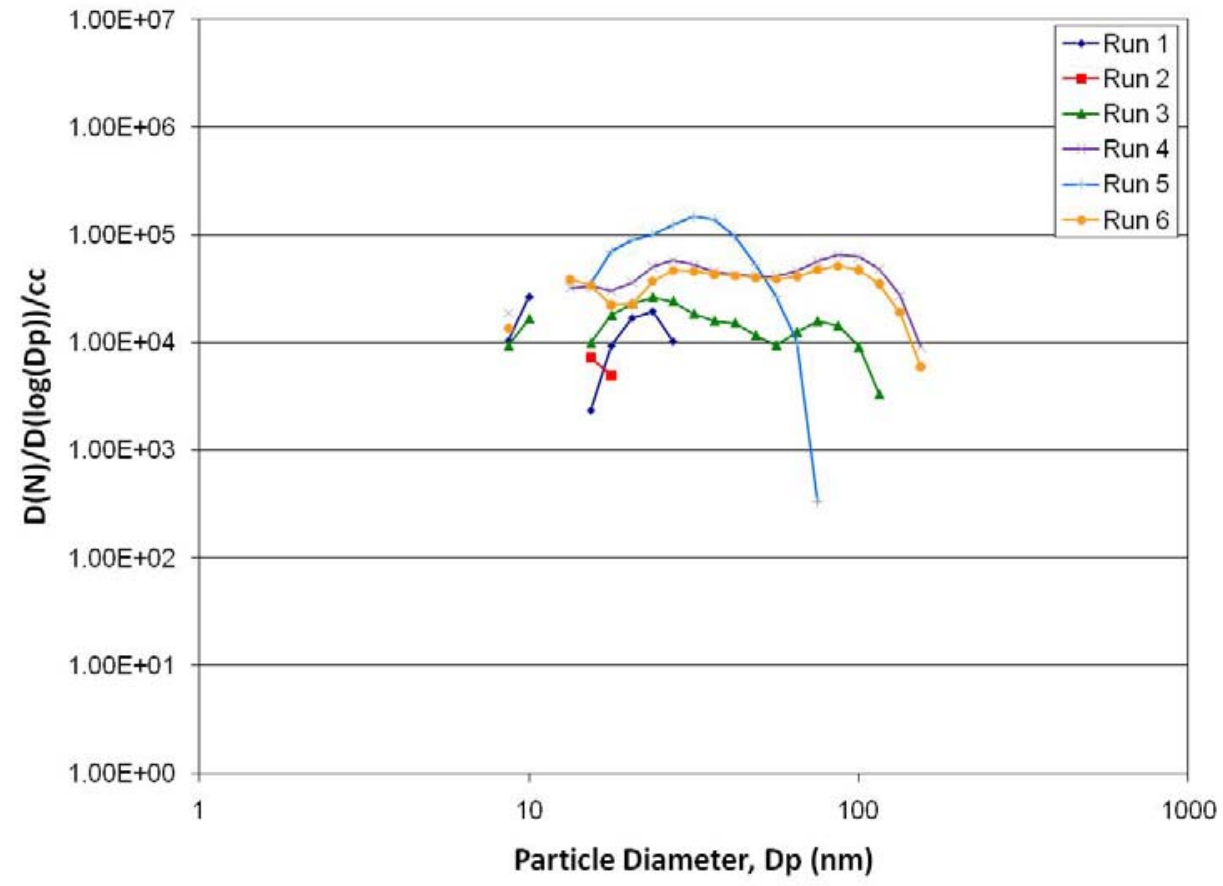

Figure 10-11: Lognormal particle size distribution for hard acceleration gasoline vehicle tests with stroller.

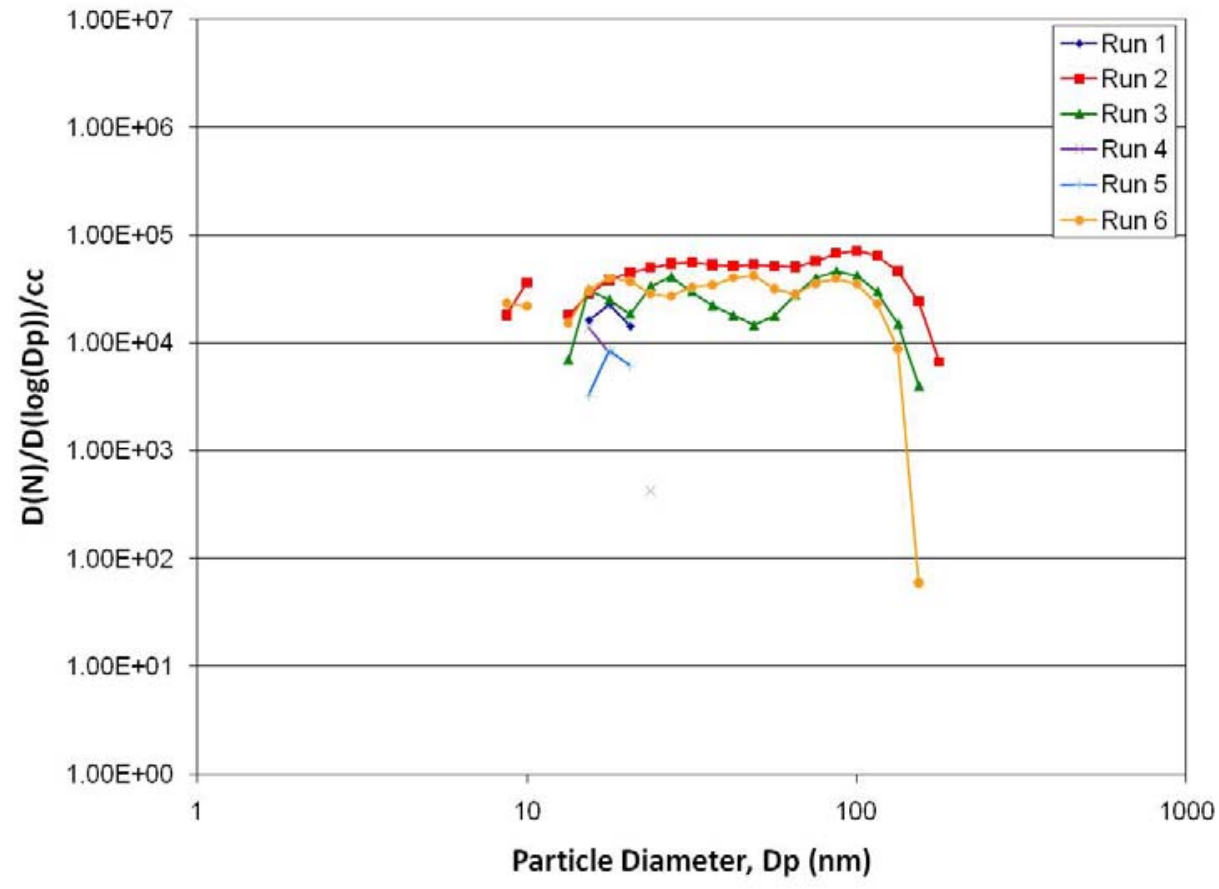

Figure 10-12: Lognormal particle size distribution for hard acceleration gasoline vehicle tests without mannequin. 


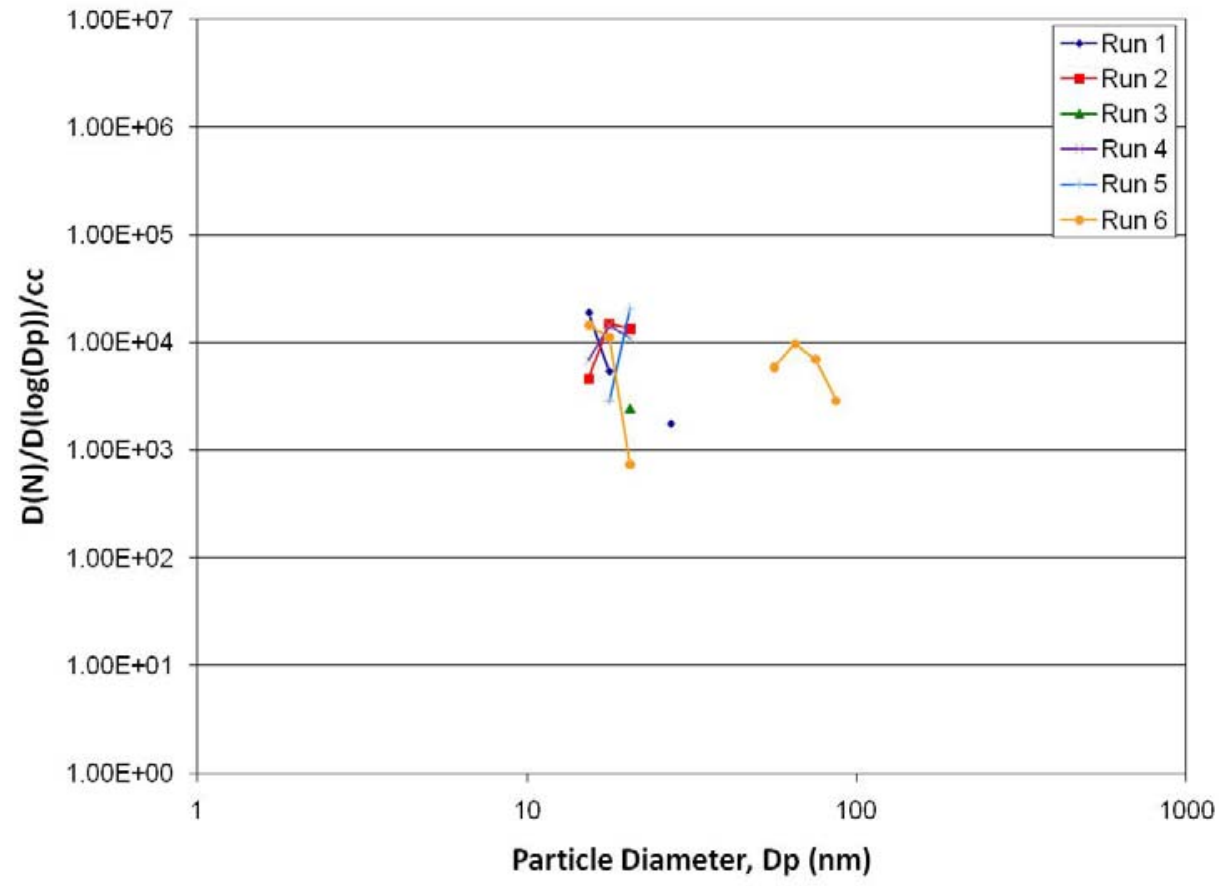

Figure 10-13: Lognormal particle size distribution for medium acceleration gasoline vehicle tests with mannequin.

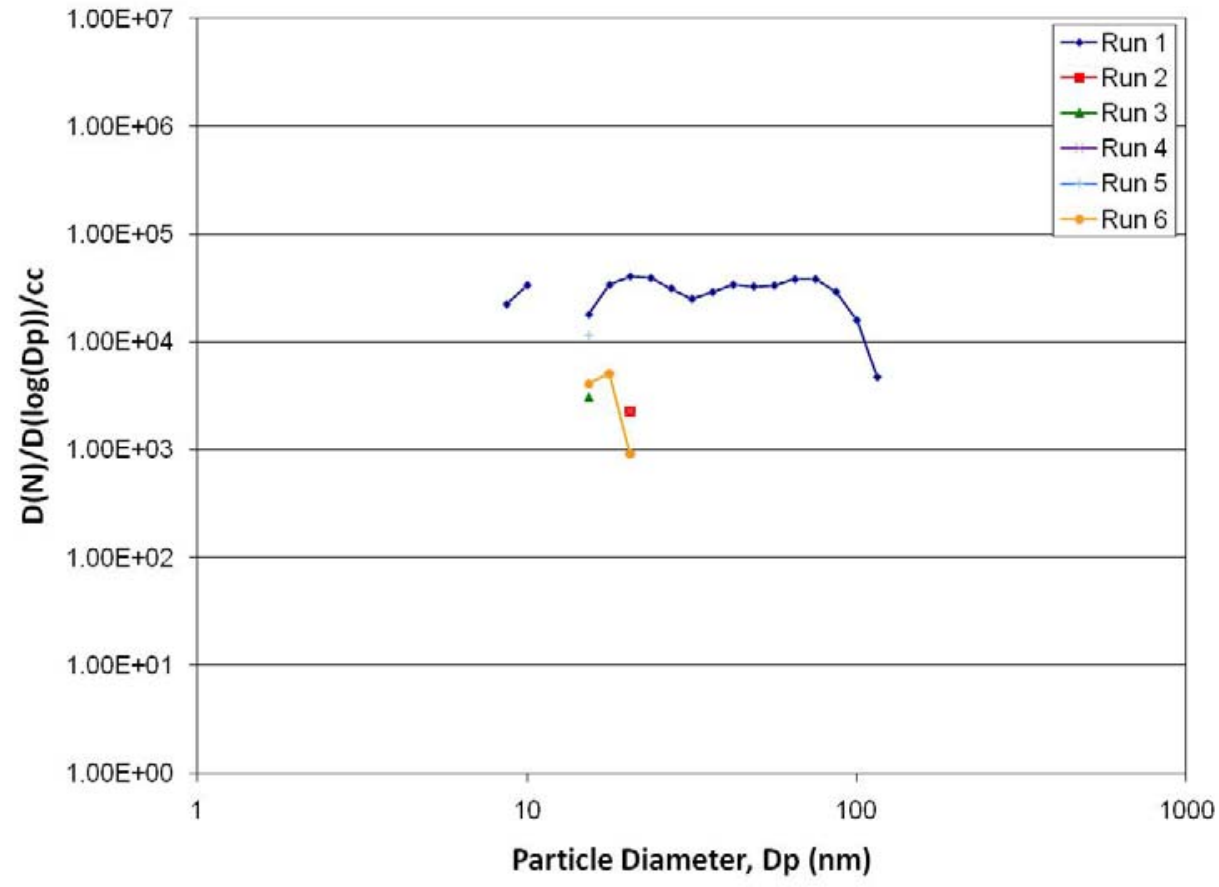

Figure 10-14: Lognormal particle size distribution for medium acceleration gasoline vehicle tests with stroller. 


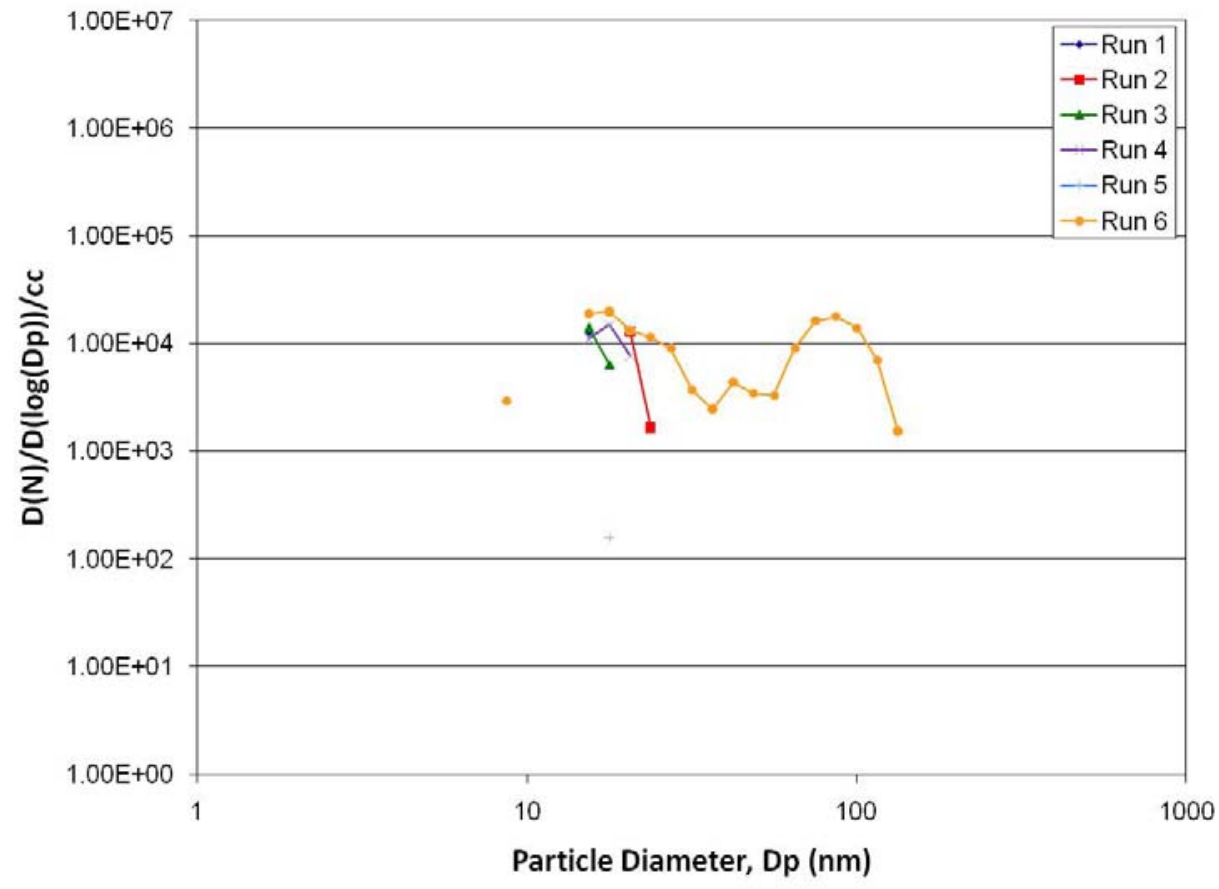

Figure 10-15: Lognormal particle size distribution for medium acceleration gasoline vehicle tests without mannequin.

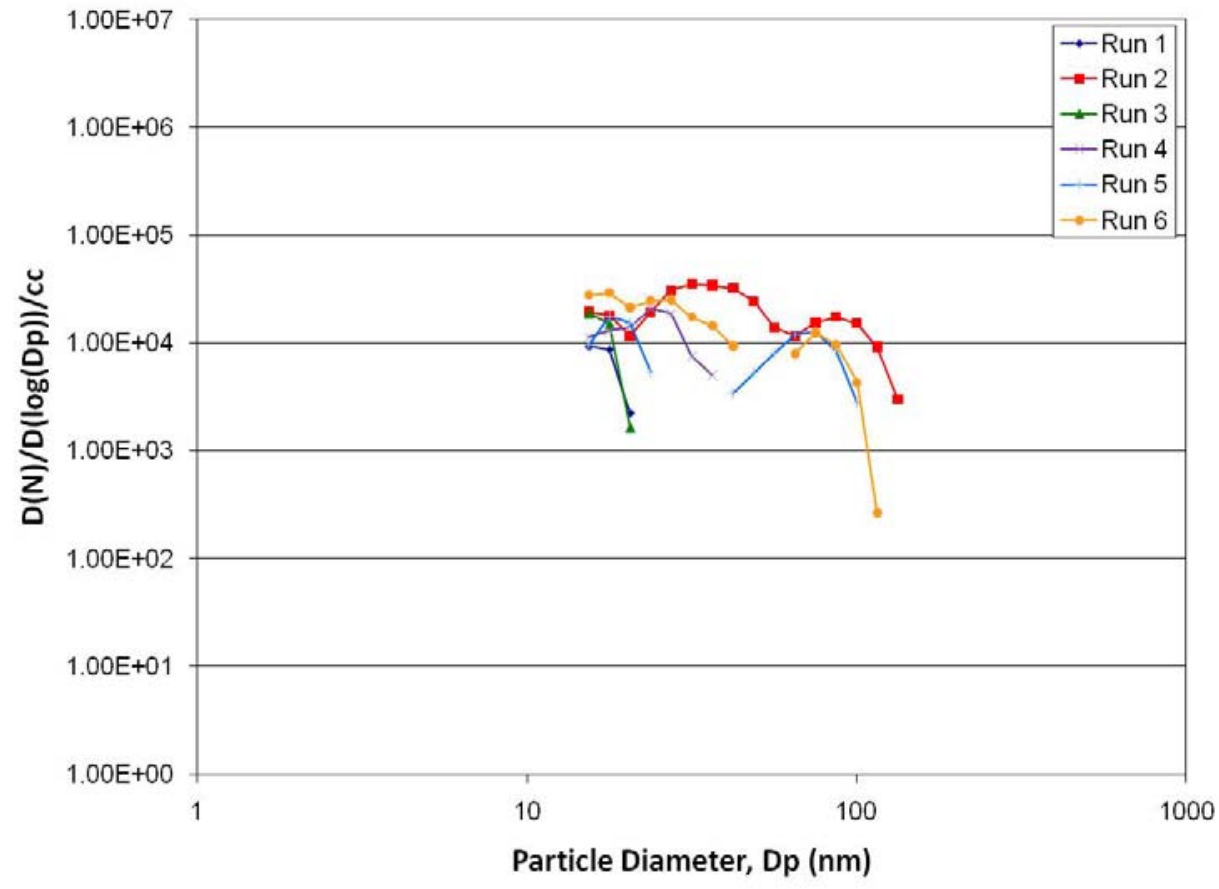

Figure 10-16: Lognormal particle size distribution for cruising gasoline vehicle tests with mannequin. 


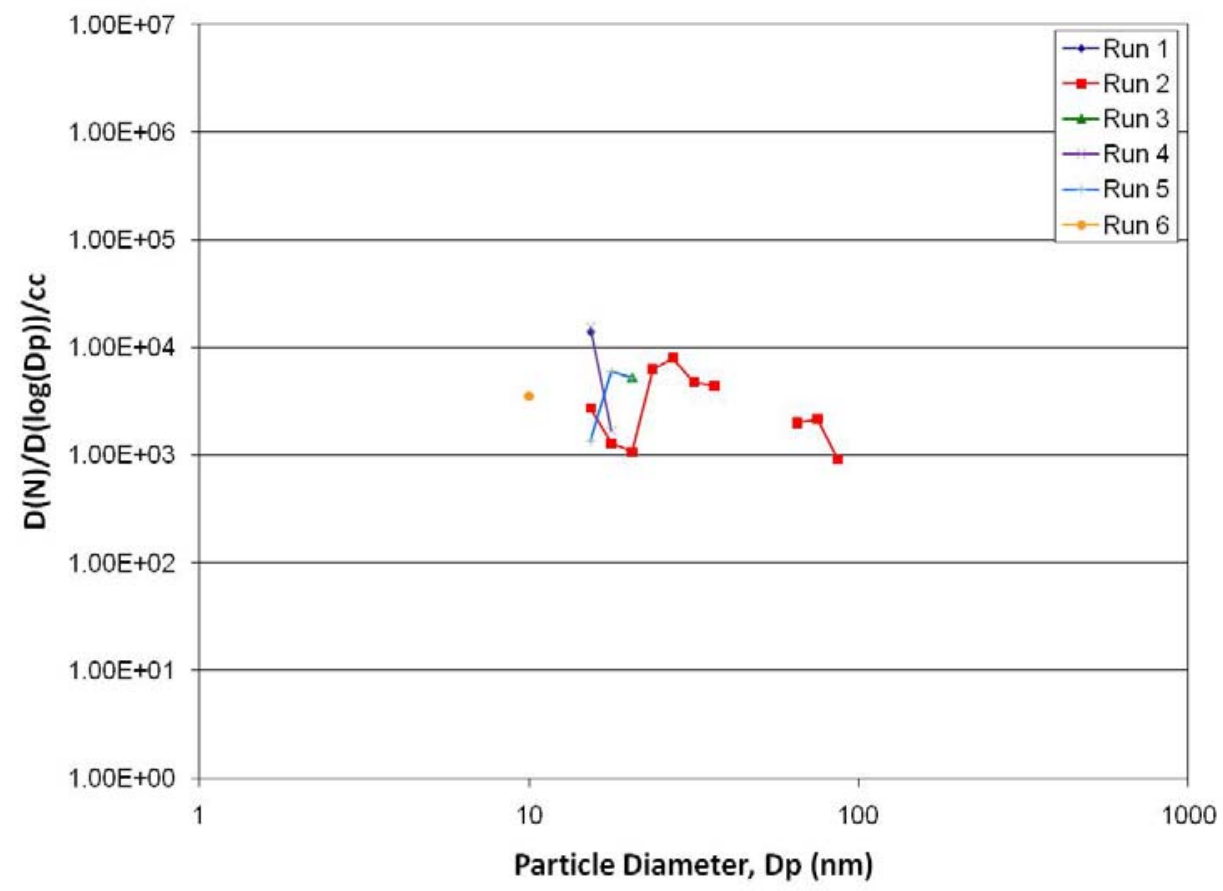

Figure 10-17: Lognormal particle size distribution for cruising gasoline vehicle tests with stroller.

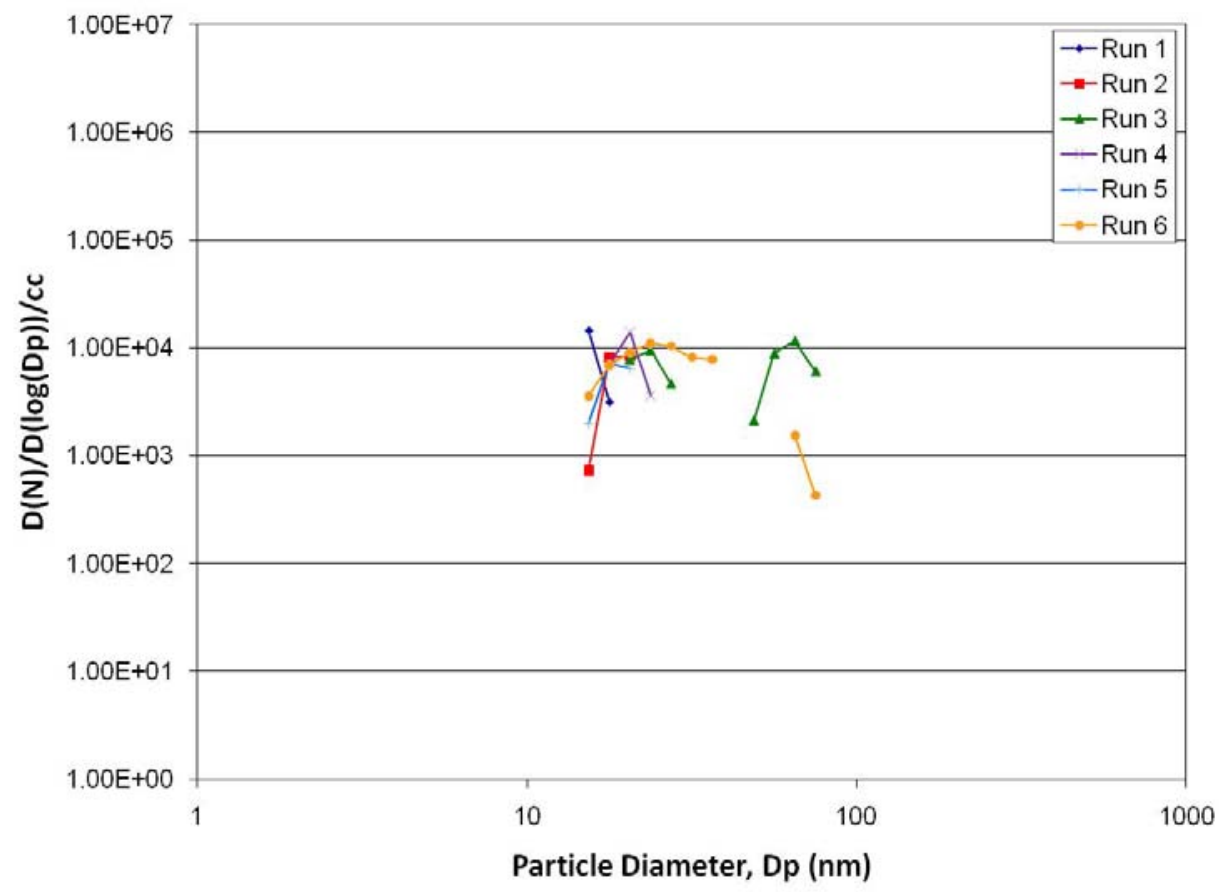

Figure 10-18: Lognormal particle size distribution for cruising gasoline vehicle tests without mannequin. 